\title{
Government Smart Card Interoperability Specification
}

Version 2.1

\section{Teresa Schwarzhoff}

Jim Dray

John Wack

Eric Dalci

Alan Goldfine

Michaela lorga 

Interoperability Specification

The National Institute of Standards and Technology 


\section{Reports on Computer Systems Technology}

The Information Technology Laboratory (ITL) at the National Institute of Standards and Technology (NIST) promotes the U.S. economy and public welfare by providing technical leadership for the Nation's measurement and standards infrastructure. ITL develops tests, test methods, reference data, proof concept implementations, and technical analysis to advance the development and productive use of information technology. ITL's responsibilities include the development of technical, physical, administrative, and management standards and guidelines for the cost-effective security and privacy of sensitive unclassified information in Federal computer systems. This Interagency Report discusses ITL's research, guidance, and outreach efforts in computer security, and its collaborative activities with industry, government, and academic organizations.

Natl. Inst. Stand. Technol. Interagency Report 6887 - 2003 Edition, 247 pages (July 2003)

Certain commercial entities, equipment, or materials may be identified in this document in order to describe an experimental procedure or concept adequately. Such identification is not intended to imply recommendation or endorsement by the National Institute of Standards and Technology, nor is it intended to imply that the entities, materials, or equipment are necessarily the best available for the purpose. Implementation of this specification or various aspects of it may be covered by U.S. and foreign patents. 
NIST IR 6887 - 2003 EDITION, GSC-IS VERSION 2.1

\section{THIS PAGE INTENTIONALLY LEFT BLANK.}




\section{Foreword}

(a) This section is non-normative and is provided for informational purposes only.

\section{(b) The Government Smart Card Initiative}

The Presidential Budget for Fiscal Year 1998 stated: "The Administration wants to adopt 'smart card' technology so that, ultimately, every Federal employee will be able to use one card for a wide range of purposes, including travel, small purchases, and building access." The General Services Administration (GSA) was requested to take the lead in developing the Federal business tools of electronic commerce and smart cards. The Federal Smart Card Implementation Plan was then developed, under which GSA implemented a pilot program to test Government smart cards and related systems. As part of the implementation plan, GSA formed the Government Smart Card Inter-Agency Advisory Board (GSC$\mathrm{IAB}$ ) to serve as a steering committee for the U.S. Government Smart Card (GSC) program.

In 1999, the National Institute of Standards and Technology (NIST) agreed to lead development of technical specifications and standards related to the GSC program. NIST represents the GSC program in industry, government, and formal standards organizations, as appropriate, to promote GSC technology. NIST is also charged with developing a comprehensive GSC conformance test program.

In May 2000, GSA awarded the Smart Access Common ID Card contracts to five prime contractors to provide smart card goods and services. Information on the use and applicability of the GSA Contract can be found at http://www.gsa.gov/smartcard.

The GSC-IAB established the Architecture Working Group (formerly known as the Technical Working Group), which consists of representatives of the contract awardees and federal agencies. The AWG, chaired and led by NIST, developed the Government Smart Card Interoperability Specification (GSC-IS), version 1.0. This specification defined the Government Smart Card Interoperability Architecture, which satisfies the core interoperability requirements of the Common Access Smart ID Card contract and the GSC Program as a whole. The AWG subsequently updated version 1.0 and released 2.0.

\section{(c) Change Management, Requirements Definition, and Interpretation of the Specification}

The GSC-IAB has the overall responsibility to develop the policy and procedures for handling revisions of the GSC-IS and any other maintenance. These procedures will be posted on the NIST smart card program web site (see Section (d)).

As additional language bindings to the Basic Services Interface (see Section 1.3) are developed, they will be added to the GSC-IS.

In the longer term, it is expected that the GSA-IAB will be the governing body for the identification of the U.S. Government's requirements. Major releases of the GSC-IS will be determined by the GSC-IAB. NISTIR 6887 will be submitted for formal standardization to the ANSI approved formal standards setting body for smart card technology.

The interpretation of the GSC-IS is the responsibility of the GSC-IAB. Interpretation issues and their resolutions will be detailed on the NIST program web site (see Section (d)).

\section{(d) Testing for Conformance}

NIST is developing a comprehensive conformance test program in support of the GSC program. Products available will be subject to a formal certification process to validate conformance to the requirements of 
the GSC-IS. The goal of the conformance tests is to determine whether or not a given Government Smart Card product conforms with the GSC Specification. Qualified laboratories will perform operational conformance testing. The GSC-IAB Conformance Committee is chaired by GSA, with representatives from the federal agencies and GSA contract awardees.

NIST is working on user guidance for achieving conformance certification for the various elements of the GSC-IS framework. This guidance will be posted at http://smartcard.nist.gov

\section{(e) NIST Government Smart Card Program Web Site}

NIST maintains a publicly accessible web site at http://smartcard.nist.gov. This page contains information on all aspects of the GSC program related to the GSC-IS, including:

- General program descriptions and updates

- The current version of the GSC-IS

- GSC-IS revision and standardization plans

- A list of errata and other changes to the last published version of the GSC-IS

- A list of interpretations and clarifications of the GSC-IS, as issued by the GSC-IAB

- Details of the GSC-IS interpretation procedures

- Details of the GSC-IS conformance-testing program. 


\section{Acknowledgements}

The authors would like to acknowledge the efforts of the original Government Smart Card Interoperability Committee; the Government Smart Card Interagency Advisory Board, composed of representatives from the public and private sectors; the General Services Administration; the prime contractors associated with the Smart Access Common ID Card contract; and the NIST smart card team. Composed of industry and government representatives, the Interoperability Committee developed the first Government Smart Card Interoperability Specification (version 1.0) during the summer of 2000.

The efforts of the GSC Architecture Work Group (formerly known as Technical Working Group) of the Government Smart Card Interagency Advisory Board are particularly recognized. Chaired by the National Institute of Standards and Technology, the AWG was responsible for reviewing the original Government Smart Card Interoperability Specification. The AWG has been a major contributor to the development of this new version of the Government Smart Card Interoperability Specification. Special recognition is extended to the AWG. 
NIST IR 6887 - 2003 EDITION, GSC-IS VERSION 2.1

\section{THIS PAGE INTENTIONALLY LEFT BLANK.}




\section{Table of Contents}

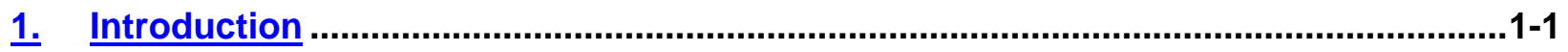

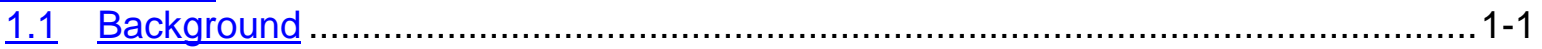

1.2 Scope, Limitations, and Applicability of the Specification.......................................1-1

1.3 Conforming to the Specification ……............................................................... 1-2

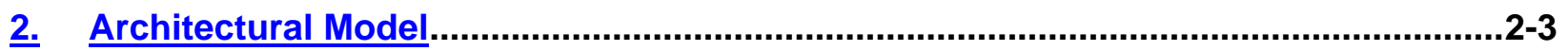

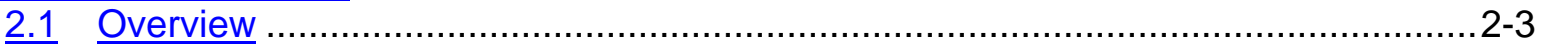

$\underline{2.2}$ Basic Services Interface Overview............................................................2-4

$\underline{2.3}$ Extended Service Interfaces Overview ……………….................................. 2-5

$\underline{2.4}$ Virtual Card Edge Interface Overview ............................................................ 2-5

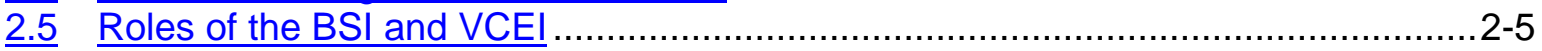

$\frac{25}{2.6}$ GSC-IS Data Model Overview...................................................................2-6

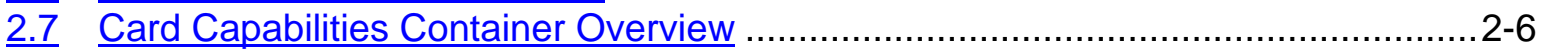

$\frac{2.8}{2.8}$ Service Provider Software Overview................................................................. $2-6$

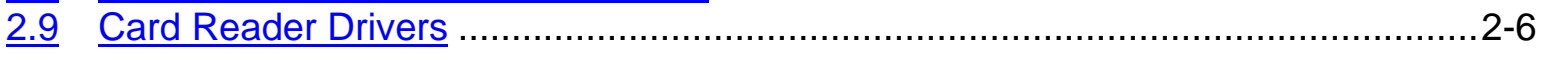

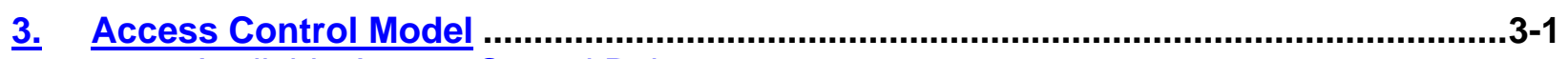

$\underline{3.1}$ Available Access Control Rules ……................................................................

$\underline{3.2}$ Determining Containers.................................................................................

$\underline{3.3}$ Establishing a Security Context........................................................................ $3-4$

$\underline{3.3 .1}$ PIN Verification ..............................................................................

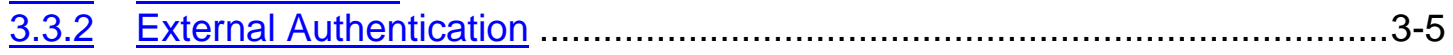

3.3.3 Secure Messaging..........................................................................

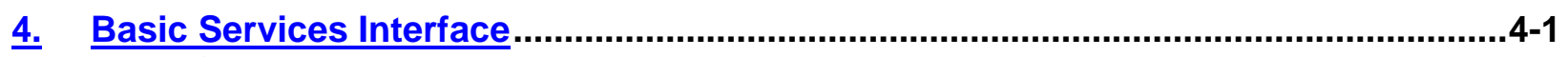

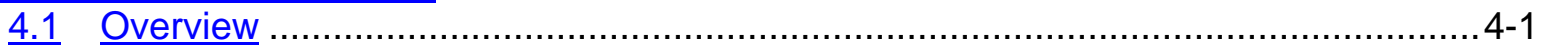

$\underline{4.2}$ Binary Data Encoding.............................................................................. 4-2

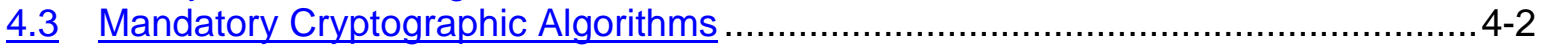

$4.4 \quad$ BSI Return Codes ...................................................................................... $4-3$

4.5 Smart Card Utility Provider Module Interface Definition ..........................................4-4

$\underline{4.5 .1}$ Pseudo IDL Definition........................................................................4-4

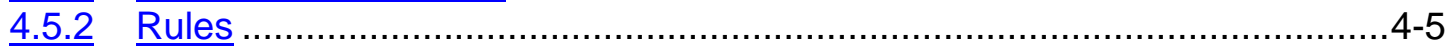

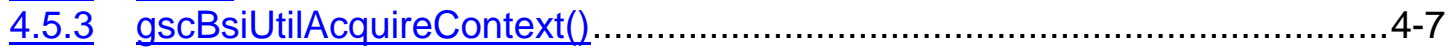

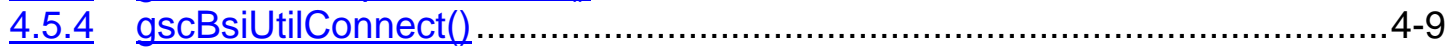

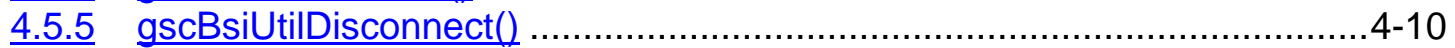

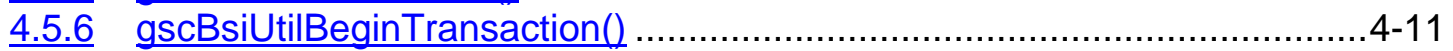

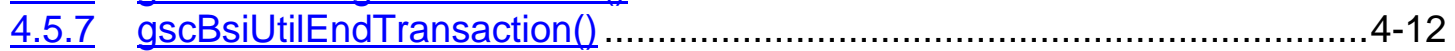

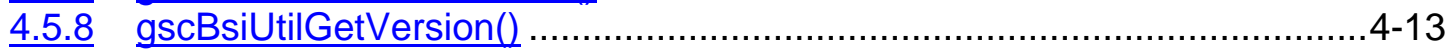

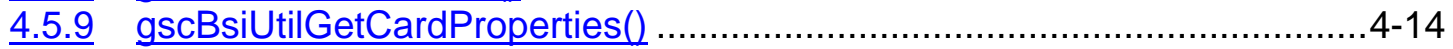

$\underline{4.5 .10}$ gscBsiUtilGetCardStatus()........................................................... 4-15

4.5.11 gscBsiUtilGetExtendedErrorText() ……………................................4-16

$\underline{4.5 .12}$ gscBsiUtilGetReaderList() ................................................................ $4-17$

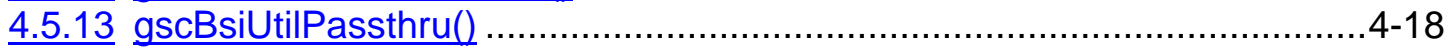

4.5.14 gscBsiUtilReleaseContext())........................................................ 4-19

4.6 Smart Card Generic Container Provider Module Interface Definition.....................4-20

4.6 .1 gscBsiGcDataCreate() .................................................................. $4-20$

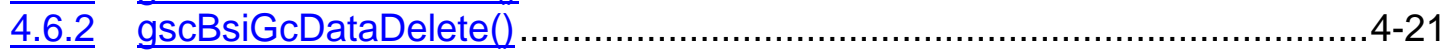

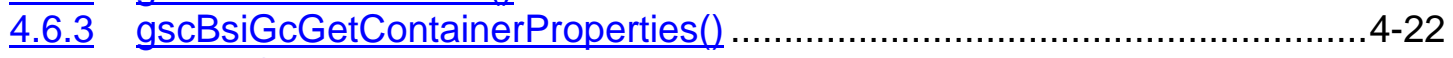

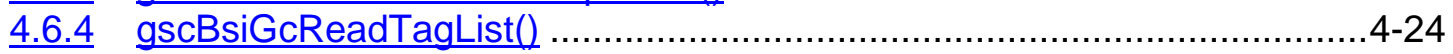

4.6.5 gscBsiGcReadValue() ……............................................................. 


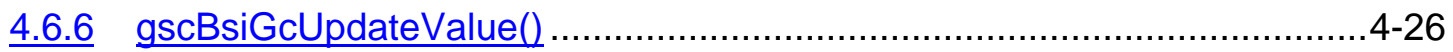

4.7 Smart Card Cryptographic Provider Module Interface Definition …........................4-27

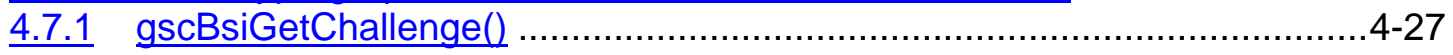

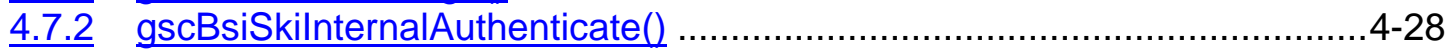

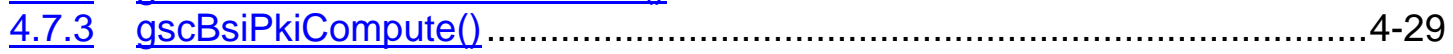

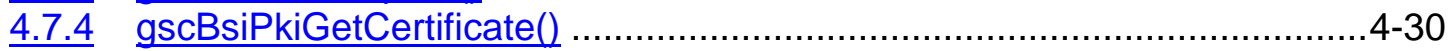

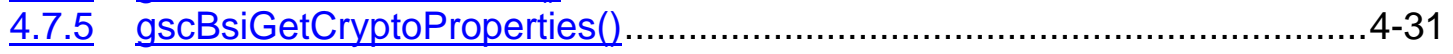

5. Virtual Card Edge Interface .................................................................................. 5-1

5.1 GSC-IS ISO Conformant APDUs …….......................................................... 5-1

5.1.1 Generic File Access APDUs.............................................................5-2

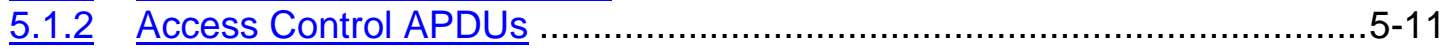

5.1.3 Public Key Operations APDUs ..........................................................5-18

5.2 Mapping Default APDUs to Native APDU Sets ……..........................................5-21

5.2.1 The CCC Command and Response Tuples...........................................5-21

5.2.2 Native APDU Mapping and CCC Grammar............................................5-21

5.2 .3 Detecting Card APDUs....................................................................5-22

5.2.4 Default Status Code Responses ……………..................................5-23

5.3 Card Edge Interface for VM Cards ...............................................................5-23

5.3.1 Virtual Machine Card Access Control Rule Configuration............................5-24

5.3.2 Virtual Machine Card Edge General Error Conditions.................................5-24

5.3.3 Common Virtual Machine Card Edge Interface Methods ............................5-25

5.3.4 Generic Container Provider Virtual Machine Card Edge Interface..............5-41

5.3.5 Symmetric Key Provider Virtual Machine Card Edge Interface..................5-44

5.3.6 Public Key Provider Virtual Machine Card Edge Interface ..........................5-48

6. Card Capabilities Container .....................................................................................6-1

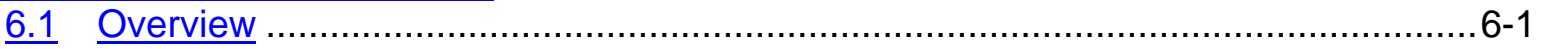

6.2 Procedure for Accessing the CCC ……………..........................................6-2

6.2 .1 General CCC Retrieval Sequence …….............................................6-2

6.2.2 Card Capabilities Container Structure ………….....................................6-4

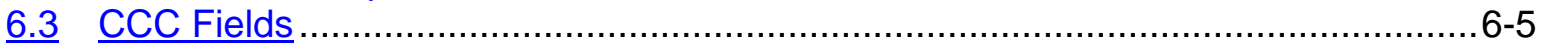

6.3.1 Card Identifier Description.................................................................6-5

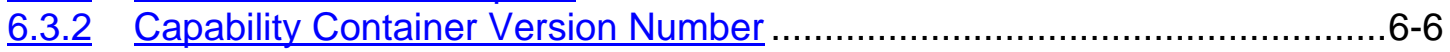

6.3.3 Capability Grammar Version Number.....................................................6-6

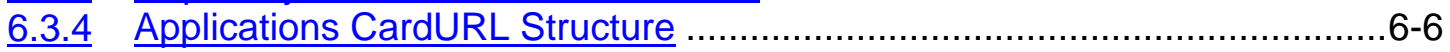

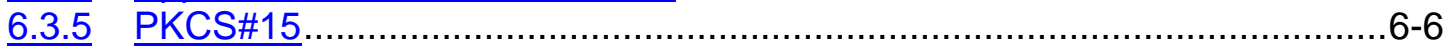

6.3.6 Registered Daa Model Number ……………….................................6-6

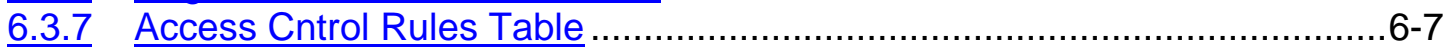

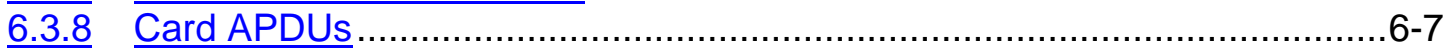

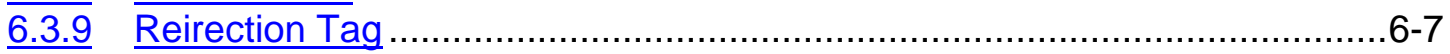

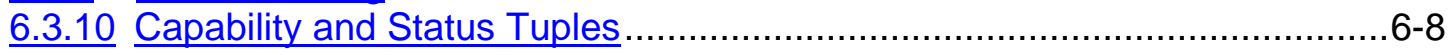

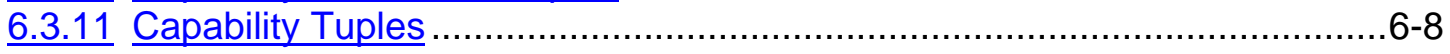

6.3.12 Prefix and Suffix Codes ....................................................................

6.3.13 Descriptor Codes............................................................................

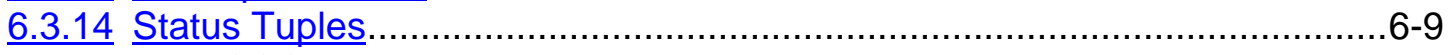

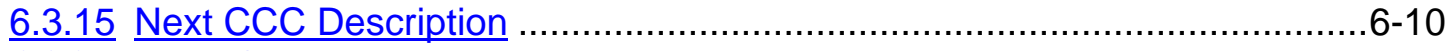

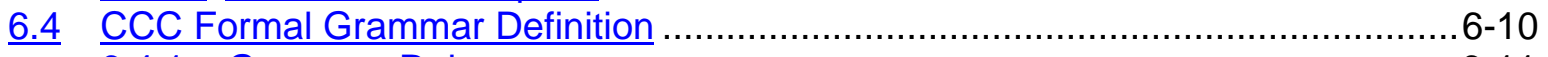

6.4.1 Grammar Rules ................................................................................6-11

6.4.2 Extended Function Codes ……............................................................6-13 


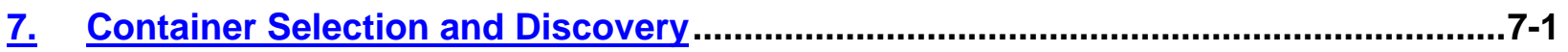

7.1 AID Abstraction: The Universal AID ……........................................................

7.2 The CCC Universal AID and CCC Applet …………........................................ 7-1

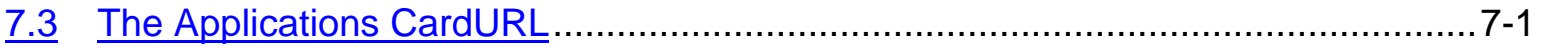

7.4 Using the Applications CardURL Structure for Container Selection..........................7-3

7.5 File System Cards: Selecting Containers..............................................................

7.6 VM Cards: Selecting Containers and Applets .................................................... $7-3$

7.7 Using the Applications CardURL Structure for Identifying Access Control Rules ....7-3

8. Data Model .............................................................................................................

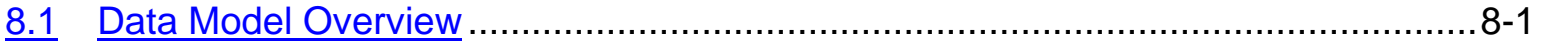

8.2 Internal Tag-Length-Value Format ............................................................... 8-1

8.3 Structure and Length Values for Cards Requiring the File System Card Edge

8.4 Structure and Length Values for Cards Requiring the Virtual Machine Card Edge...8-2

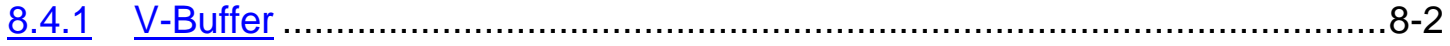




\section{Appendices}

1. Introduction

1.1 Background .......................................................................................... $1-1$

1.2 Scope, Limitations, and Applicability of the Specification..................................... 1-1

1.3 Conforming to the Specification ……............................................................... 1-2

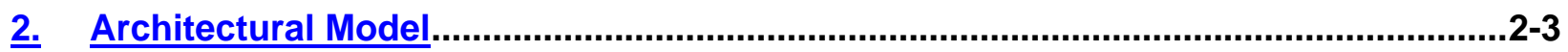

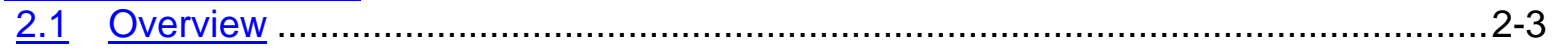

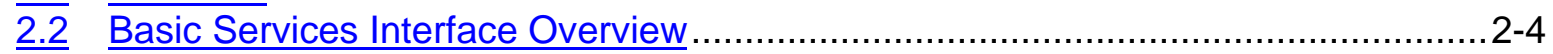

$\underline{2.3}$ Extended Service Interfaces Overview ……………….................................... 2-5

$\underline{2.4}$ Virtual Card Edge Interface Overview ............................................................ 2-5

$\frac{2.5}{2.5}$ Roles of the BSI and VCEI ...................................................................... 2-5

$\frac{25}{2.6}$ GSC-IS Data Model Overview...................................................................2-6

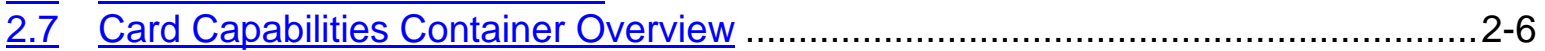

$\frac{2.8}{2.8}$ Service Provider Software Overview................................................................. $2-6$

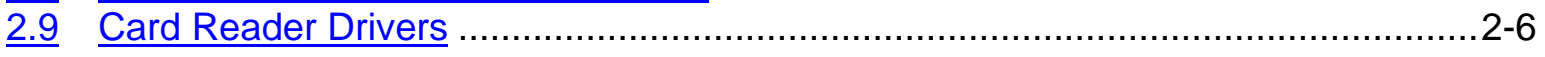

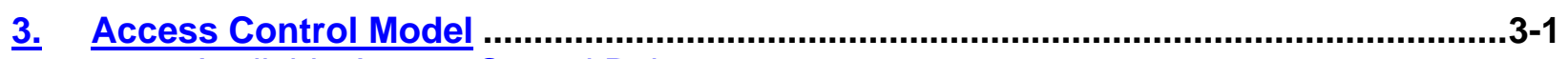

$\underline{3.1}$ Available Access Control Rules ……................................................................

$\underline{3.2}$ Determining Containers.................................................................................

$\underline{3.3}$ Establishing a Security Context........................................................................ $3-4$

$\underline{3.3 .1}$ PIN Verification ..............................................................................

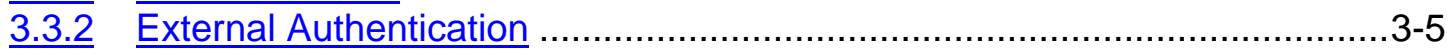

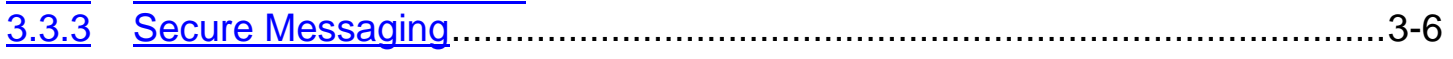

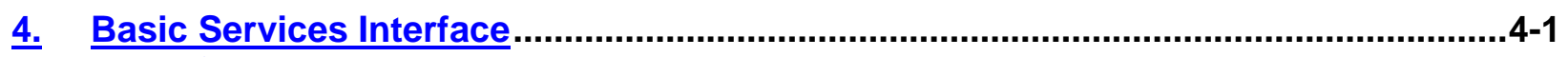

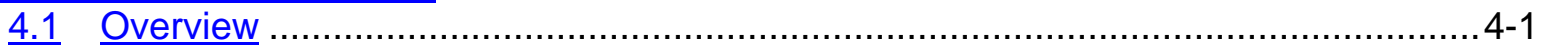

4.2 Binary Data Encoding............................................................................. 4-2

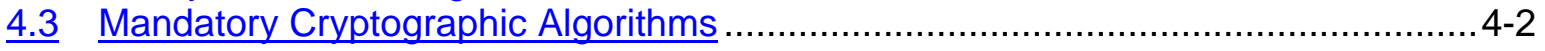

$4.4 \quad$ BSI Return Codes ...................................................................................... $4-3$

4.5 Smart Card Utility Provider Module Interface Definition ..........................................4-4

$\underline{4.5 .1}$ Pseudo IDL Definition.........................................................................4-4

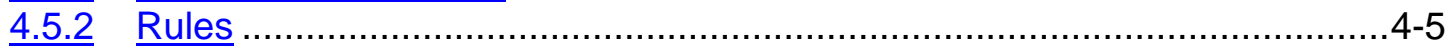

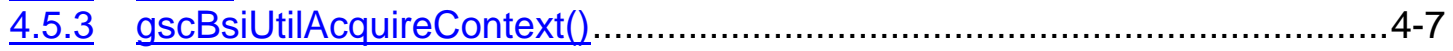

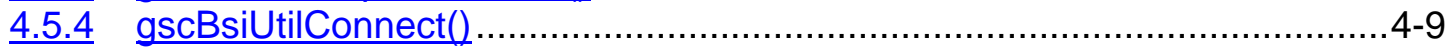

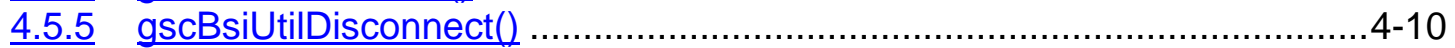

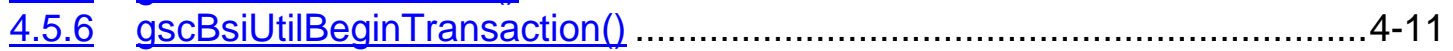

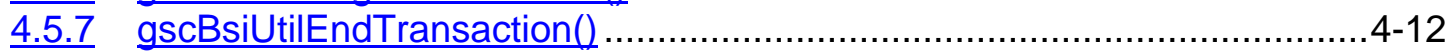

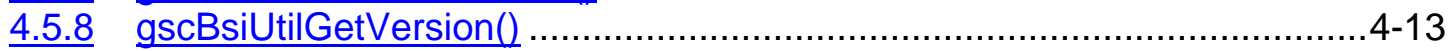

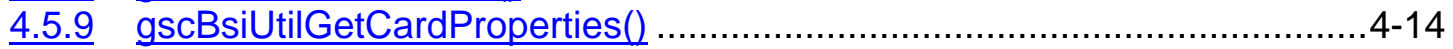

$\underline{4.5 .10}$ gscBsiUtilGetCardStatus()........................................................... 4-15

4.5.11 gscBsiUtilGetExtendedErrorText() ……………................................4-16

$\underline{4.5 .12}$ gscBsiUtilGetReaderList() ................................................................ $4-17$

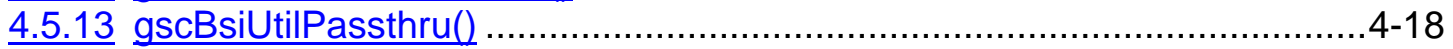

4.5.14 gscBsiUtilReleaseContext())........................................................ 4-19

4.6 Smart Card Generic Container Provider Module Interface Definition.....................4-20

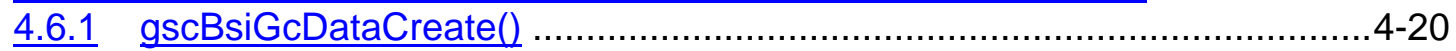

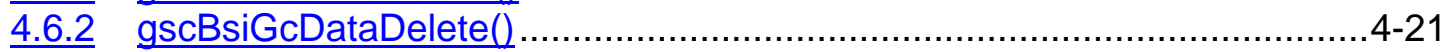

$\underline{4.6 .3}$ gscBsiGcGetContainerProperties() ............................................... 4-22

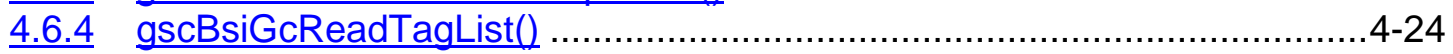

4.6.5 gscBsiGcReadValue() ……............................................................. 
4.6.6 gscBsiGcUpdateValue() ............................................................ 4-26

4.7 Smart Card Cryptographic Provider Module Interface Definition …........................4-27

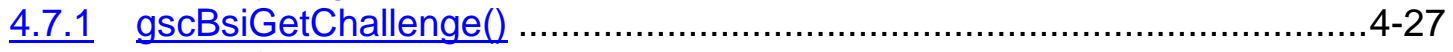

$\underline{4.7 .2}$ gscBsiSkilnternalAuthenticate() …............................................. 4-28

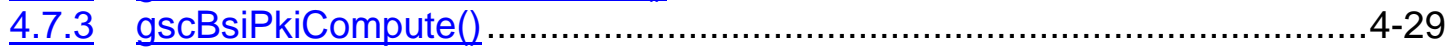

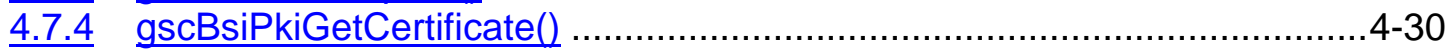

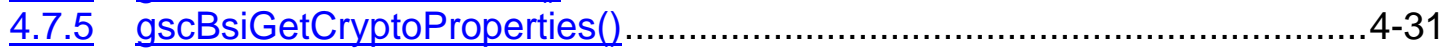

5. Virtual Card Edge Interface .................................................................................. 5-1

5.1 GSC-IS ISO Conformant APDUs ……………................................................ 5-1

5.1.1 Generic File Access APDUs.............................................................5-2

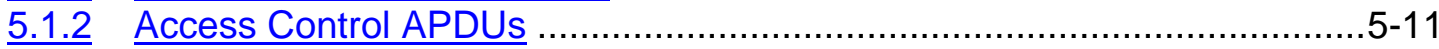

5.1.3 Public Key Operations APDUs ..........................................................5-18

5.2 Mapping Default APDUs to Native APDU Sets ..................................................5-21

5.2.1 The CCC Command and Response Tuples............................................5-21

5.2.2 Native APDU Mapping and CCC Grammar..............................................5-21

5.2 .3 Detecting Card APDUs....................................................................5-22

5.2.4 Default Status Code Responses ……………..................................5-23

5.3 Card Edge Interface for VM Cards ................................................................ 5-23

5.3.1 Virtual Machine Card Access Control Rule Configuration...........................5-24

5.3.2 Virtual Machine Card Edge General Error Conditions.................................5-24

5.3.3 Common Virtual Machine Card Edge Interface Methods ............................5-25

5.3.4 Generic Container Provider Virtual Machine Card Edge Interface..............5-41

5.3.5 Symmetric Key Provider Virtual Machine Card Edge Interface...................5-44

5.3.6 Public Key Provider Virtual Machine Card Edge Interface ..........................5-48

6. Card Capabilities Container …..................................................................................6-1

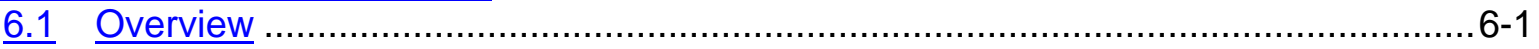

6.2 Procedure for Accessing the CCC ……..................................................... 6-2

6.2.1 General CCC Retrieval Sequence ………….....................................6-2

6.2.2 Card Capabilities Container Structure …………….................................6-4

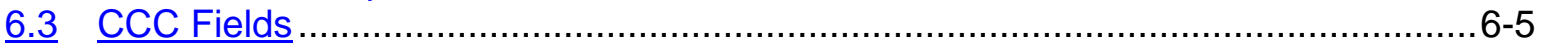

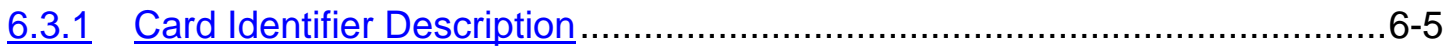

6.3 .2 Capability Container Version Number ………………………………...6-6

6.3.3 Capability Grammar Version Number.....................................................6-6

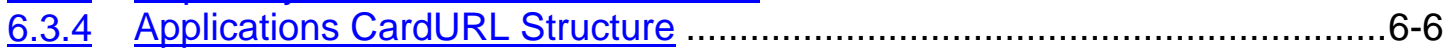

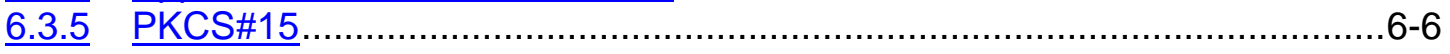

6.3.6 Registered Daa Model Number ……………….................................6-6

6.3 .7 Access Cntrol Rules Table ....................................................................6-7

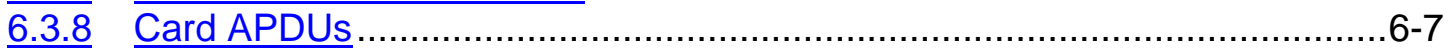

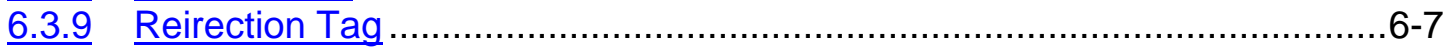

$\frac{6.3 .10}{6.31}$ Capability and Status Tuples .................................................................6-8

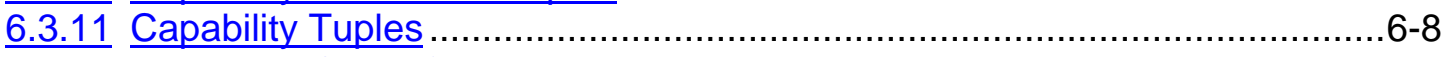

$\underline{6.3 .12}$ Prefix and Suffix Codes ..................................................................... 6-9

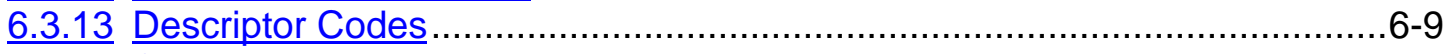

6.3.14 Status Tuples................................................................................

6.3.15 Next CCC Description ………...........................................................6-10

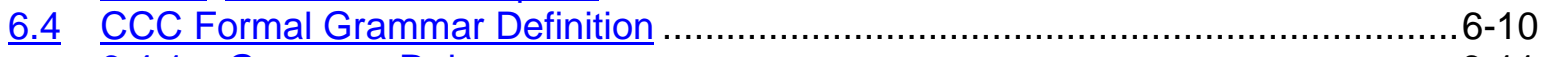

6.4.1 Grammar Rules ................................................................................6-11

6.4.2 Extended Function Codes ……............................................................6-13 


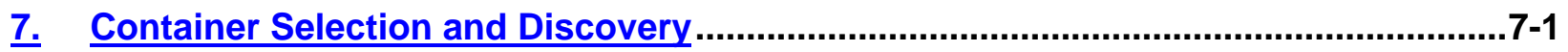

7.1 AID Abstraction: The Universal AID ……........................................................

7.2 The CCC Universal AID and CCC Applet .....................................................

7.3 The Applications CardURL .........................................................................

7.4 Using the Applications CardURL Structure for Container Selection..........................7-3

7.5 File System Cards: Selecting Containers ..............................................................

7.6 VM Cards: Selecting Containers and Applets ..................................................... 7-3

7.7 Using the Applications CardURL Structure for Identifying Access Control Rules ....7-3

8. Data Model .............................................................................................................

8.1 Data Model Overview .................................................................................. 8-1

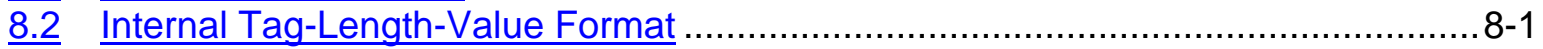

8.3 Structure and Length Values for Cards Requiring the File System Card Edge

8.4 Structure and Length Values for Cards Requiring the Virtual Machine Card Edge...8-2

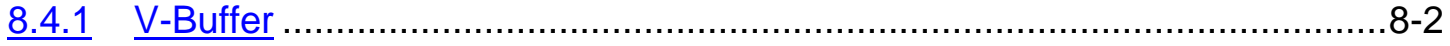




\section{List of Appendices}

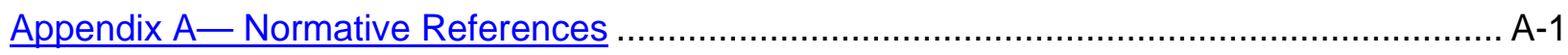

Appendix B-Informative References.................................................................... B-1

Appendix C- GSC Data Model................................................................................

Appendix D- DoD Common Access Card (CAC) Data Model ............................................ D-1

Appendix E-C Language Binding for BSI Services....................................................... E-1

Appendix F- Java Language Binding for BSI Services ................................................ F-1

Appendix G-Contactless Smart Card Requirements ...................................................

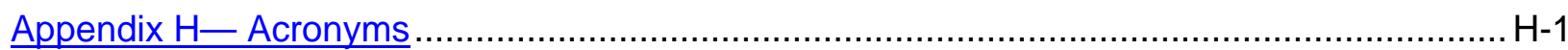


NIST IR 6887 - 2003 EDITION, GSC-IS VERSION 2.1

\section{Figures and Tables}

Figure 2-1: The GSC-IS Architectural Model ………...................................................... 2-4

Figure 6-1: The Card Capability Container .......................................................................6-1

Figure 6-2: Location of the CCC Elementary File in a file system card .................................6-2

Figure 6-3: Shift Tuple Sequence (SL: shift level) .......................................................6-14

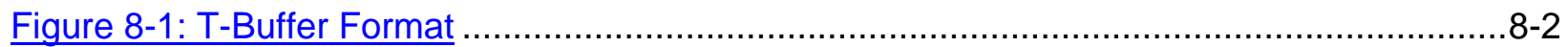

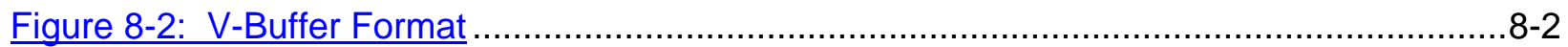

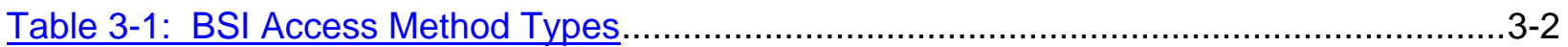

Table 3-2: BSI Access Control Rule Types ..................................................................

Table 3-3: ACRs for Generic Container Provider Module Services ......................................... 3-4

Table 3-4: ACRs for Cryptographic Provider Module Services .............................................. 3-4

Table 4-1: BSI Return Codes..................................................................................

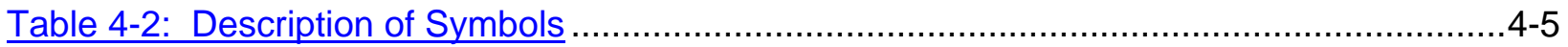

Table 4-3: Mapping Pseudo IDL to Java......................................................................

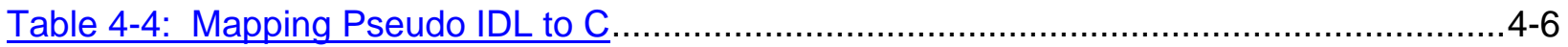

Table 5-1: GSC-IS APDU Set ..................................................................................

Table 5-2: APDU Command and Response Structure ……..............................................5-2

Table 5-3: APDU Command and Response Structure ……............................................5-2

Table 5-4: Generic File Access APDUs ....................................................................

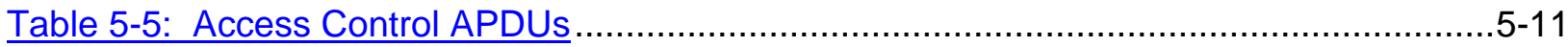

Table 5-6: Algorithm Identifiers for Authentication APDUs ..................................................5-12

Table 5-7: Public Key Operations APDUs ………….................................................

Table 5-8: CARD APDUs Values ...............................................................................

Table 5-9: GSC-IS Status Code Responses.................................................................

Table 5-10: Virtual Machine Card Edge APDUs ……......................................................5-23

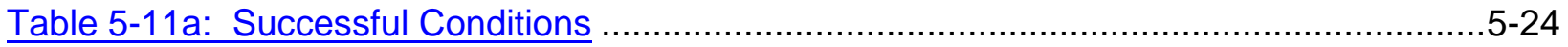

Table 5-11b: General Error Conditions ..................................................................

Table 5-12: Common VM APDUs ..............................................................................

Table 5-13: ACRs assigned to the Common VM CEI …….............................................. $5-26$

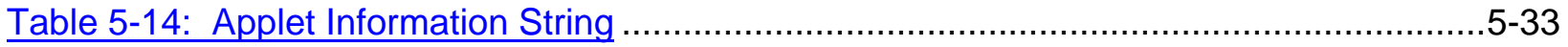

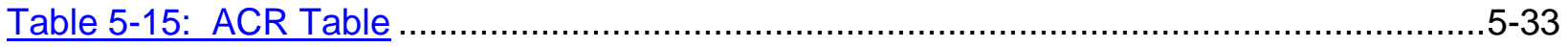

Table 5-16: Applet/Object ACR Table ........................................................................... 5-34

Table 5-17: Access Method Provider Table .........................................................................5-34 
Table 5-18: Service Applet Table .................................................................................. 5 -36

Table 5-19: Applet/Object ACR table for a Single Object ……......................................5-36

Table 5-20: Access Method Provider Table ....................................................................

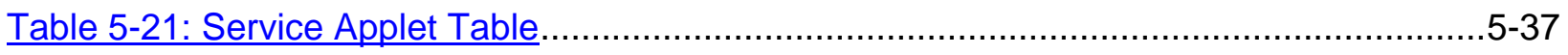

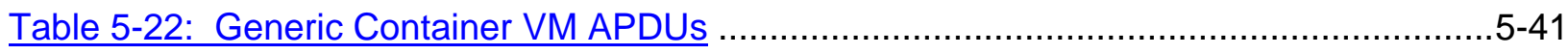

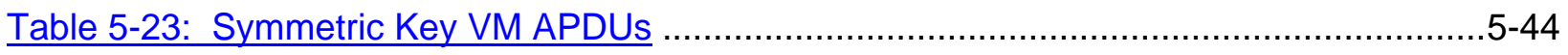

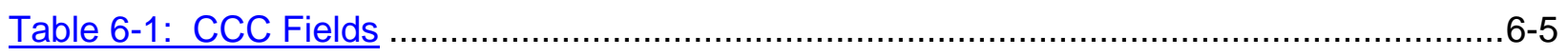

Table 6-2: Tuple Byte Descriptions ............................................................................ 6-8

Table 6-3: Parameter and Function Codes .................................................................... 6-9

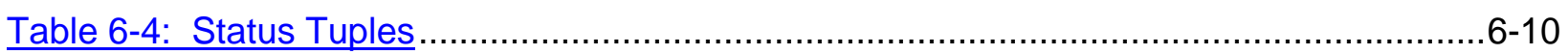

Table 6-5: Standard Status Code Responses ...........................................................

Table 6-6: Default vs. Schlumberger DF APDU...........................................................

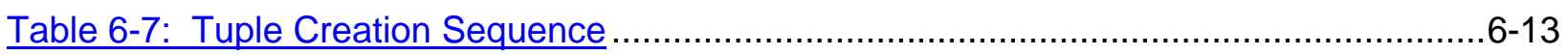

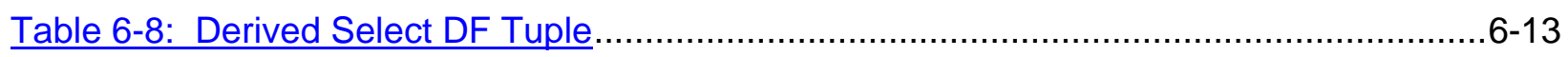

Table 6-9: Example of Extended Function Code .......................................................6-14

Table 6-10: Descriptor Codes ................................................................................

Table E-1: BSI functions using the discovery method .................................................. E-2 
NIST IR 6887 - 2003 EDITION, GSC-IS VERSION 2.1

\section{THIS PAGE INTENTIONALLY LEFT BLANK.}




\section{Introduction}

\section{$1.1 \quad$ Background}

A typical configuration for a smart card system consists of a host computer with one or more smart card readers attached to hardware communications ports. Smart cards can be inserted into the readers, and software running on the host computer communicates with these cards using a protocol defined by ISO 7816-4 [ISO4] and 7816-8 [ISO8]. The ISO standard smart card communications protocol defines Application Protocol Data Units (APDU) that are exchanged between smart cards and host computers. This APDU based interface is referred to as the "virtual card edge" and the two terms are used interchangeably.

Client applications have traditionally been designed to communicate with ISO smart cards using the APDU protocol through low-level software drivers that provide an APDU transport mechanism between the client application and a smart card. Smart card families can implement the APDU protocol in a variety of ways, so client applications must have intimate knowledge of the APDU set of the smart card they are communicating with. This is generally accomplished by programming a client application to work with a specific card, since it would not be practical to design a client application to accommodate the different APDU sets of a large number of smart card families.

The tight coupling between client applications and smart card APDU sets has several drawbacks. Applications programmers must be thoroughly familiar with smart card technology and the complex APDU protocol. If the cards that an application is hard coded to use become commercially unavailable, the application must be redesigned to use different cards. Customers also have less freedom to select different smart card products, since their applications will only work with one or a small number of similar cards.

This Government Smart Card Interoperability Specification (GSC-IS) provides solutions to a number of the interoperability challenges associated with smart card technology. The original version of the GSC-IS (version 1.0, August 2000) was developed by the GSC Interoperability Committee led by the General Services Administration (GSA) and the National Institute of Standards and Technology (NIST), in association with the GSA Smart Access Common Identification Card contract.

\subsection{Scope, Limitations, and Applicability of the Specification}

The GSC-IS defines an architectural model for interoperable smart card service provider modules, compatible with both file system cards and virtual machine cards. Smart cards using both the T=0 and $\mathrm{T}=1$ [ISO3] communications protocols are supported. The GSC-IS includes a Basic Services Interface (BSI), which addresses interoperability of a core set of smart card services at the interface layer between client applications and smart card service provider modules. The GSC-IS also defines a mechanism at the card edge layer for interoperation with smart cards that use a wide variety of APDU sets, including both file system cards and virtual machine cards.

Interoperability is not addressed for the following areas:

- Smart card initialization

- Cryptographic key management

- Communications between smart cards and card readers 
- Communications between smart card readers and host computer systems.

\subsection{Conforming to the Specification}

A smart card service provider module implementation that claims conformance to the GSC-IS must implement each of the following:

- The Architectural Model, as defined in Chapter 2

- The Access Control Model, as defined in Chapter 3

- The Basic Services Interface, as defined in Chapter 4

- The Virtual Card Edge Interface, as defined in $\underline{\text { Chapter } 5}$

- The Card Capabilities Container, as defined in Chapter 6

- Container Naming, as defined in $\underline{\text { Chapter } 7}$

- Support for both of the Container Data Models defined in $\underline{\text { Chapter } 8}$ and the appropriate Appendices

- At least one language binding for BSI Services, as defined in the Appendices.

A smart card that claims conformance to the GSC-IS must support each of the following:

- The Architectural Model as it relates to smart cards, i.e., as defined in sections $\underline{1}, \underline{4}, \underline{5}$, and $\underline{6}$ of Chapter 2

- The Access Control Model, as defined in Chapter 3

- Either the file system card edge interface or the VM card edge interface, as defined in Chapter 5

- The Card Capabilities Container, as defined in $\underline{\text { Chapter } 6}$

- Container Naming, as defined in $\underline{\text { Chapter } 7}$

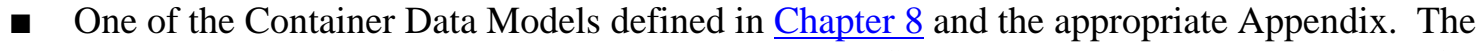
Access Control File and associated SEIWG string defined in Appendix $\mathrm{C}$ are mandatory for contact-type GSC cards, and the SEIWG container defined in Appendix G is mandatory for contactless GSC cards.

As used in this document, the conformance keywords "shall" and "must" (which are interchangeable) denote mandatory features of the GSC-IS. The keyword "should" denotes a feature that is recommended but not mandatory, while the keyword "may" denotes a feature whose presence or absence does not preclude conformance. 


\section{Architectural Model}

\subsection{Overview}

The GSC-IS provides interoperability at two levels: the service call level and the card command (APDU) level. A brief explanation of these interoperability levels follows:

- Service Call Level: This level is concerned with functional calls required to obtain various services from the card (e.g., encryption, authentication, digital signatures, etc.). The GSC-IS addresses interoperability at this level by defining an Applications Programming Interface (API) called the Basic Services Interface (BSI) that defines a common high level model for smart card services. The module that implements the BSI and provides an interoperable set of smart card services to client applications is called the Service Provider Module (SPM). These services are logically divided into three modules that provide utility, secure data storage, and cryptographic services. Since an SPM generally will be implemented through a combination of hardware and software, the software component of the SPM is referred to as the Service Provider Software (SPS).

- Card Command Level: This level is concerned with the exact APDUs (ISO4) that are sent to the card to obtain the required service. The GSC-IS addresses interoperability at this level by defining the API called the Virtual Card Edge Interface (VCEI) that consists of a cardindependent standard set of APDUs that support the functions defined in the BSI and implemented by the SPM.

The SPM is a combination of both these levels and it includes:

- SPS, implementing both BSI and VCEI interfaces

- Smart card reader driver

- Smart card reader

- GSC-IS conformant smart card

Certain data sets need to be available in the card to support the interoperability provided by the BSI and VCEI. To ensure that there is a standard format (or schema) for storing these data sets, and to enable uniform access and interpretation, the GSC-IS defines Data Models (DM). These Data Models provide data portability across GSC-IS conformant card implementations, ensuring that a core set of data elements is available on all cards. The storage entities for various categories of data sets are called containers. One of these containers, the Card Capability Container (CCC), describes the differences between a smart card's native APDU set and the standard APDU set defined by the VCEI. An SPS retrieves a smart card's CCC and uses it to perform the translation between the VCEI and the card's native APDU set. The GSC-IS accommodates any smart card whose APDU set can be mapped to the VCEI via a CCC definition.

The components of the GSC-IS architecture are presented in Figure 2-1 and are further described in

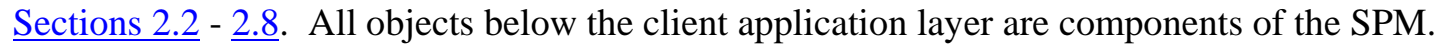




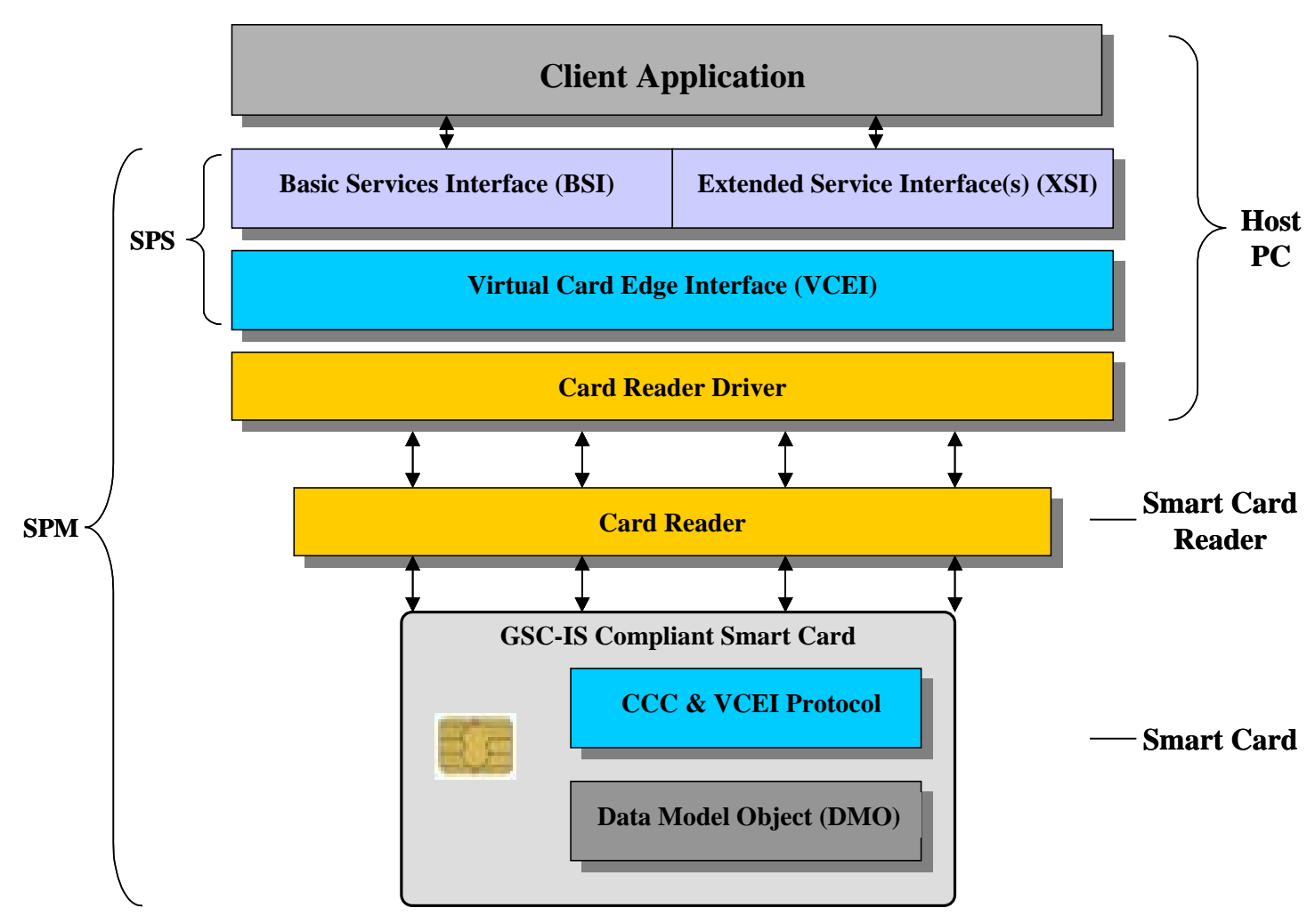

Figure 2-1: The GSC-IS Architectural Model

\subsection{Basic Services Interface Overview}

All Smart Card Service Provider Modules shall implement the BSI. The BSI is logically organized into three provider modules:

- Utility Provider Module: Provides utility services for obtaining a list of available card readers, establishing and terminating logical connections with a smart card, etc.

- Generic Container Provider Module: Provides a unified abstraction of the storage services of smart cards, presenting applications with a simple interface for managing generic containers of data elements in Tag/Length/Value format [ISO4].

- Cryptographic Provider Module: Provides fundamental cryptographic services such as random number generation, authentication, and digital signature generation.

The capabilities of a given SPM depend on the smart card available to the SPM when a client application requests a service through a BSI call. In cases where a service is not available, the BSI call shall return an error code indicating that the requested service is not available. For example, a user may insert a smart card that does not have public key cryptographic capabilities and then perform an operation that causes a client application to request a digital signature calculation from the associated SPM. Since the smart card cannot provide this service, the BSI shall return a "service not available" error code to the client application. 


\subsection{Extended Service Interfaces Overview}

Because the BSI is not a complete operational interface, real world SPM implementations may support additional functionality outside the BSI domain. Because the BSI provides an interoperable interface, it is unable to address the varying operational requirements. Therefore, real world SPM implementations may support additional functionality outside the BSI domain. An SPM may therefore include an Extended Service Interface (XSI) that provides non-interoperable, but operationally required, functions. Since XSIs are implementation and application specific, they are accommodated by the GSC-IS architectural model but are not defined in the GSC-IS. Card initialization and cryptographic key management are examples of functions that must currently be implemented in the XSI domain.

\subsection{Virtual Card Edge Interface Overview}

ISO 7816-4 [ISO4] defines a hierarchical file system structure for smart cards. Smart cards that conform to ISO 7816-4 [ISO4] are therefore known as "file system" cards. The Card Operating System program of a file system card is usually hard coded into the logic of the smart card integrated circuit during the manufacturing process and cannot be changed thereafter.

In recent years other smart card architectures have been created that allow developers to load executable programs onto smart cards after the cards have been manufactured. As one example, JavaCard ${ }^{\mathrm{TM}}$ [JAVA] defines a Java Virtual Machine (VM) specification for smart card processors. Developers can load compiled Java applets onto a smart card containing the JavaCard ${ }^{\mathrm{TM}} \mathrm{VM}$, programmatically changing the behavior of the card.

A virtual machine card is one that can be extended by loading executable programs after the card has been manufactured. This Specification uses the term "virtual machine smart card" in the general sense. A virtual machine smart card can theoretically be programmed to support any communications protocol, including the APDU based protocols of the ISO 7816-4 [ISO4] and 7816-8 [ISO8] standard.

The GSC-IS VCEI defines default sets of interoperable APDU level commands for both virtual machine and file system smart cards. The SPS of an SPM shall use the information provided by a smart card's CCC to map that card's native APDU set to the VCEI default set. The VCEI shall consist of:

- A card edge definition for file system cards

- A card edge definition for VM cards, composed of three providers:

- A generic container provider

- A symmetric key (SKI) cryptographic service provider

- A public key infrastructure (PKI) cryptographic service provider.

\subsection{Roles of the BSI and VCEI}

The service provider modules of the BSI are a higher level abstraction of the card level providers. Standardization at the VCEI layer establishes interoperability between any GSC conformant SPS and any GSC conformant smart card. Similarly, standardization at the BSI layer establishes interoperability between any GSC conformant application and any GSC conformant SPS. Vendor neutrality is assured because GSC smart cards are interchangeable at the VCEI and GSC SPSs are interchangeable at both the BSI and VCEI. 


\subsection{GSC-IS Data Model Overview}

Each GSC-IS conformant smart card shall conform to a GSC-IS Data Model. GSC-IS Data Models define the set of containers and data elements within each container for cards supporting that Data Model. The GSC-IS defines two Data Models: the GSC Data Model (Appendix C) (formerly referred to as the J.8 Data Model in GSC-IS v1.0) and the U.S. Department of Defense Common Access Card Data Model (Appendix D). The following containers are mandatory in either Data Model:

- CCC for contact and contactless cards and

- Access control file with SEIWG [SEIW] string for contact cards or

- SEIWG container and SEIWG [SEIW] string for contactless cards.

The remaining containers and data elements are optional. However, if an implementation requires any of the containers and data elements defined in the Data Models, the containers and data elements must conform to the Data Model definitions. Data Model requirements are presented in $\underline{\text { Chapter } 8 .}$

Containers are accessed through the Generic Container Provider Module of the BSI. Access to the containers are subject to the Access Control Rules (ACR) defined in $\underline{\text { Chapter } 3}$.

This document uses the terms "file," "container," and "object" synonymously.

\subsection{Card Capabilities Container Overview}

Each GSC-IS conformant card shall carry a Card Capabilities Container. The CCC is one of the mandatory containers that must be present in all GSC-IS Data Models. The purpose of the CCC is to describe the differences between a given card's APDU set and the APDU set defined by the GSC-IS Virtual Card Edge Interface. The GSC-IS provides standard mechanisms for retrieving a CCC from a smart card (Section 6.2). Once the CCC for a particular card is obtained, software on the host computer (specifically, the SPS) uses this information to translate between the VCEI and the card's native APDU set. Deviations from the card's Data Model structure are represented in a CCC.

The CCC allows each GSC-IS conformant smart card to carry the information needed by the SPS to communicate with that card. This general mechanism for dynamically translating APDU sets eliminates the need to distribute, install, and maintain card specific APDU level drivers on host computer systems.

The rules for constructing a valid CCC are defined in Section 6.3. All GSC-IS smart cards shall contain a $\mathrm{CCC}$ that conforms to this specification.

\subsection{Service Provider Software Overview}

The SPS component of an SPM shall implement the BSI and the VCEI. It is responsible for retrieving CCCs from cards, using this information to translate between the smart card's native APDU set and the VCEI, and for handling the details of APDU level communications with the card. SPS implementations work with a particular card reader driver layer that transports APDUs between the SPS and the smart card.

\subsection{Card Reader Drivers}

The GSC-IS does not address interoperability between smart card readers and host computer systems. Several specifications already exist in this area, including the Personal Computer Smart Card (PC/SC, 
[PCSC]) specification and the OpenCard Framework (OCF, [OCF]). The choice of card reader driver software is influenced to some degree by the operating environment, although PC/SC and OCF have been ported to various operating systems.

Because card reader driver solutions are available and several of these have been widely adopted, the GSC-IS allows developers the freedom to choose any card reader driver that provides the reader level services required by the SPS layer including:

- Transport of "raw" (unprocessed) APDUs between the SPS layer and the smart card,

- Functions to provide a list of available readers,

- And to establish and terminate logical connections to cards inserted into readers.

Proprietary card reader drivers can also be used as long as they provide the raw APDU transport and card reader management functions required by an SPS. Some applications may have unique requirements that mandate a special purpose card reader. For example, the configuration required by a physical access control application may not be able to accommodate a PC/SC or OCF card reader driver layer and would therefore require a custom card reader driver.

The decision not to include a card reader driver layer specification in the GSC-IS has important consequences. This implies a pair-wise relationship between an SPS and the card reader driver. An SPS implementation works with a specific card reader driver and is constrained to operate with the card readers supported by that driver. The degree of interoperability between card readers and host computer systems is entirely determined by the card reader driver component.

In cases where an industry standard card reader driver component is chosen, it is possible to take advantage of existing conformance test programs and select from a range of commercially available, conformant card readers. If a special purpose (proprietary) card reader driver is chosen, these options may not be available. In some cases proprietary card reader drivers work only with proprietary card reader designs, and may therefore require development of special purpose conformance test programs. 
NIST IR 6887 - 2003 EDITION, GSC-IS VERSION 2.1

\section{THIS PAGE INTENTIONALLY LEFT BLANK.}




\section{Access Control Model}

The smart card services and containers provided by a SPM are subject to a set of Access Control Rules (ACR). ACRs are defined for each card service and default container when a GSC-IS conformant smart card is initialized. The card level service providers are responsible for enforcing these ACRs and shall not provide a given service until the client application has fulfilled the applicable access control requirements. The GSC-IS specifies a discovery mechanism that allows client applications to determine the ACRs for a specific service provider or container.

It is important to note that an SPS acts as a transport and reformatting mechanism for the exchange of authentication data, such as PINs and cryptograms, between client applications and smart cards. When a client application and smart card service provider establish a security context, the primary job of the SPS is to reformat BSI level authentication structures into APDU level VCEI structures and vice versa. The current GSC-IS model does not include a mechanism for authenticating an SPS, and the SPS is not responsible for enforcing ACRs.

\subsection{Available Access Control Rules}

The ACRs available at the BSI level are as follows:

- Always: The corresponding service can be provided without restrictions.

- Never: The corresponding service can never be provided.

- External Authenticate: The corresponding service can be provided only after a GET CHALLENGE and subsequent EXTERNAL AUTHENTICATE APDUs.

- PIN Protected: The corresponding service is provided if and only if the verification code of the PIN associated with the service has been provided in the current card session.

- PIN Always: The corresponding service can be provided only if its associated PIN code has been verified immediately before each unique service request.

- External Authenticate or PIN: Either one of the two controls gives access to the service. This allows for a cardholder validation when a PIN pad is available and for an external authentication when no PIN pad is available. Or, this provides an authentication method when the application cannot be trusted to perform an external authentication and to protect the external authentication key.

- External Authenticate then PIN: The two methods must be chained successfully before access to the service is granted. This allows the authentication of both the client application and the user.

- External Authenticate and PIN: The two methods must be chained successfully before access to the service is granted. Order of the methods is not important.

- PIN then External Authenticate: The PIN presentation is followed by an External Authentication.

- Secure Channel (GP): The corresponding service can be provided only through a Secure Channel managed by a Global Platform [GLOB] Secure Messaging layer.

- Update Once: A target object can only be updated once during its lifetime. 
- Secure Channel (ISO): The corresponding service can be provided through a Secure Channel managed by an ISO [ISO4],[ISO8] Secure Messaging layer.

BSI-level ACRs are a logical combination of primitive access methods. The BSI-level access methods and associated hexadecimal values are summarized in the Table 3-1. Hexadecimal values are assigned to the unAccessMethodType member of the BSIAuthenticator structure defined in Section 4.5.3.

Table 3-1: BSI Access Method Types

\begin{tabular}{|l|c|l|}
\hline \multicolumn{1}{|c|}{ Access Method Type } & Value & \multicolumn{1}{c|}{ Meaning } \\
\hline BSI_AM_XAUTH & 0x02 & External Authentication. \\
\hline BSI_AM_SECURE_CHANNEL_GP & 0x04 & Secure Channel (Global Platform) \\
\hline BSI_AM_PIN & 0x06 & PIN code is required \\
\hline BSI_AM_SECURE_CHANNEL_ISO & 0x0B & Secure Channel (ISO 7816-4) \\
\hline
\end{tabular}

The BSI-level ACRs and associated hexadecimal values are summarized in Table 3-2. Hexadecimal values are returned in the ACRType member of the BSIACr structure defined in Section 4.6.3. The BSIACr structure is present in the members of the GCacr structure defined in Section 4.6.3 and the CRYPTOacr strucuture defined in Section 4.7.5.

Table 3-2: BSI Access Control Rule Types

\begin{tabular}{|l|c|c|c|l|}
\hline $\begin{array}{c}\text { Access Control Rule } \\
\text { Type } \\
\text { (ACRType) }\end{array}$ & Access Method List & $\begin{array}{c}\text { Logical } \\
\text { Relation } \\
\text { between } \\
\text { AMs }\end{array}$ & Value & \multicolumn{1}{|c|}{ Meaning } \\
\hline BSI_ACR_ALWAYS & - & - & $0 \times 00$ & $\begin{array}{l}\text { No access control rule is } \\
\text { required }\end{array}$ \\
\hline BSI_ACR_NEVER & - & - & $0 \times 01$ & Operation is never possible \\
\hline BSI_ACR_XAUTH & BSI_AM_XAUTH & - & $0 \times 02$ & External Authentication \\
\hline $\begin{array}{l}\text { BSI_ACR_XAUTH_OR } \\
\text {-PIN }\end{array}$ & $\begin{array}{l}\text { BSI_AM_XAUTH, } \\
\text { BSI_AM_PIN }\end{array}$ & OR & $0 \times 03$ & $\begin{array}{l}\text { The object method can be } \\
\text { accessed either after an External } \\
\text { Authentication or after a } \\
\text { successful PIN presentation }\end{array}$ \\
\hline $\begin{array}{l}\text { BSI_SECURE_CHANN } \\
\text { EL_GP }\end{array}$ & $\begin{array}{l}\text { BSI_AM_SECURE_ } \\
\text { CHANNEL_GP }\end{array}$ & - & $0 \times 04$ & $\begin{array}{l}\text { Secure Channel (Global } \\
\text { Platform) }\end{array}$ \\
\hline $\begin{array}{l}\text { BSI_ACR_PIN_ALWA } \\
\text { YS }\end{array}$ & BSI_AM_PIN & - & $0 \times 05$ & $\begin{array}{l}\text { PIN must be verified immediately } \\
\text { prior to service request }\end{array}$ \\
\hline BSI_ACR_PIN & BSI_AM_PIN & - & $0 \times 06$ & PIN code is required \\
\hline $\begin{array}{l}\text { BSI_ACR_XAUTH_TH } \\
\text { EN_PIN }\end{array}$ & $\begin{array}{l}\text { BSI_AM_XAUTH, } \\
\text { BSI_AM_PIN }\end{array}$ & AND & $0 \times 07$ & $\begin{array}{l}\text { External Authentication followed } \\
\text { by a PIN presentation }\end{array}$ \\
\hline $\begin{array}{l}\text { BSI_ACR_UPDATE_O } \\
\text { NCE }\end{array}$ & - & - & $0 \times 08$ & $\begin{array}{l}\text { The target object can only be } \\
\text { updated once during its lifetime }\end{array}$ \\
\hline
\end{tabular}




\begin{tabular}{|c|c|c|c|c|}
\hline $\begin{array}{c}\text { Access Control Rule } \\
\text { Type } \\
\text { (ACRType) }\end{array}$ & Access Method List & $\begin{array}{c}\text { Logical } \\
\text { Relation } \\
\text { between } \\
\text { AMs }\end{array}$ & Value & Meaning \\
\hline $\begin{array}{l}\text { BSI_ACR_PIN_THEN } \\
\text { _XAUTH }\end{array}$ & $\begin{array}{l}\text { BSI_AM_PIN, } \\
\text { BSI_AM_XAUTH }\end{array}$ & AND & $0 \times 09$ & $\begin{array}{l}\text { PIN presentation followed by } \\
\text { External Authentication }\end{array}$ \\
\hline $\begin{array}{l}\text { Reserved for } \\
\text { future use }\end{array}$ & - & - & $0 \times 0 A$ & RFU \\
\hline $\begin{array}{l}\text { BSI_SECURE_CHANN } \\
\text { EL_ISO }\end{array}$ & $\begin{array}{l}\text { BSI_AM_SECURE_ } \\
\text { CHANNEL_ISO }\end{array}$ & - & $0 \times 0 B$ & Secure Channel (ISO 7816-4) \\
\hline $\begin{array}{l}\text { BSI_ACR_XAUTH_AN } \\
\text { D_PIN }\end{array}$ & $\begin{array}{l}\text { BSI_AM_XAUTH, } \\
\text { BSI_AM_PIN }\end{array}$ & AND & $0 \times 0 C$ & $\begin{array}{l}\text { PIN presentation AND External } \\
\text { Authentication in any order are } \\
\text { required. }\end{array}$ \\
\hline Reserved for future use & - & - & $\begin{array}{l}\text { OxOD- } \\
\text { OxFF }\end{array}$ & RFU \\
\hline
\end{tabular}

The External Authentication method shall conform with ISO 7816-4 [ISO4] and 7816-8 [ISO8]. The mandated cryptographic algorithm is DES3-ECB [DES], with a double length key-size 16 bytes and a challenge of 8 bytes. This method is described in Section 3.3.2.

The ACR for the Secure Channel implies cryptographic operations performed at the APDU level. A passthrough function is provided in the BSI ( $\underline{\text { Section 4.5.13) }}$ ) to allow applications to create a secure channel and operate inside this channel.

\subsection{Determining Containers}

Applications can retrieve the ACR that must be fulfilled to access a specific service or container. ACR retrieval processes are defined for each provider module as follows:

- Utility Service Provider Module: No access control is applied.

- Generic Container Service Provider Module: ACRs for generic container services are encoded in the GCacr structure returned by the function gscBsiGcGetContainerProperties () .

- Cryptographic Service Provider Module: ACRs for cryptographic services are encoded in the CRYPTOacr structure returned by the function gscBsiGetCryptoProperties ().

Each of the services associated with a provider module have a different set of allowable ACRs. When a provider module is created (instantiated), the module creator must assign the ACRs for each of the services provided by the module from the set of supported ACRs, listed in Tables 3-3 and 3-4. 
Table 3-3: ACRs for Generic Container Provider Module Services

\begin{tabular}{|c|c|}
\hline Service & ACR supported \\
\hline gscBsiGcDataCreate() & $\begin{array}{l}\text { BSI_ACR_ALWAYS } \\
\text { BSI_ACR_NEVER } \\
\text { BSI_ACR_PIN } \\
\text { BSI_ACR_XAUTH }\end{array}$ \\
\hline gscBsiGcDataDelete() & $\begin{array}{l}\text { BSI_ACR_ALWAYS } \\
\text { BSI_ACR_NEVER } \\
\text { BSI_ACR_PIN } \\
\text { BSI_ACR_XAUTH }\end{array}$ \\
\hline gscBsiGcReadTagList() & $\begin{array}{l}\text { BSI_ACR_ALWAYS } \\
\text { BSI_ACR_PIN } \\
\text { BSI_ACR_XAUTH }\end{array}$ \\
\hline gscBsiGcReadValue () & $\begin{array}{l}\text { BSI_ACR_ALWAYS } \\
\text { BSI_ACR_PIN } \\
\text { BSI_ACR_XAUTH }\end{array}$ \\
\hline gscBsiGcUpdateValue() & $\begin{array}{l}\text { BSI_ACR_ALWAYS } \\
\text { BSI_ACR_NEVER } \\
\text { BSI_ACR_PIN } \\
\text { BSI_ACR_XAUTH } \\
\text { BSI_ACR_UPDATE_ONCE }\end{array}$ \\
\hline gscBsiGcGetContainerProperties () & BSI_ACR_ALWAYS \\
\hline
\end{tabular}

Table 3-4: ACRs for Cryptographic Provider Module Services

\begin{tabular}{|c|c|}
\hline Service & ACR supported \\
\hline gscBsiGetChallenge() & BSI_ACR_ALWAYS \\
\hline gscBsiskiInternalAuthenticate () & $\begin{array}{l}\text { BSI_ACR_ALWAYS } \\
\text { BSI_ACR_PIN } \\
\text { BSI_ACR_XAUTH }\end{array}$ \\
\hline gscBsipkiCompute() & $\begin{array}{l}\text { BSI_ACR_ALWAYS } \\
\text { BSI_ACR_PIN } \\
\text { BSI_ACR_PIN_ALWAYS } \\
\text { BSI_ACR_XAUTH }\end{array}$ \\
\hline gscBsipkiGetCertificate() & $\begin{array}{l}\text { BSI_ACR_ALWAYS } \\
\text { BSI_ACR_PIN } \\
\text { BSI_ACR_XAUTH }\end{array}$ \\
\hline gscBsiGetCryptoProperties () & BSI_ACR_ALWAYS \\
\hline
\end{tabular}

Note: When using the gscBsipkiCompute () function for signature operation, it is highly recommended that the implementation require BSI_ACR_PIN_ALWAYS for access control.

\subsection{Establishing a Security Context}

Once a client application has determined the ACR associated with a service or a container, it must establish a security context with the card. To fulfill the ACR for a container or service, the application builds a BSIAuthenticator data structure and passes it in a call to the gscBsiUtilAcquireContext () function.

Establishing a security context involves authentication of the parties involved in the service exchange. These parties include the user executing the client application, the client application itself, and the smart 
card. The GSC-IS ACRs are based on three general authentication mechanisms: PIN Verification, External Authentication, and Secure Messaging.

The External Authentication method assumes that the authentication key has been formerly distributed to both parties (client application and smart card) in a secure way.

It is important to note that at the smart card level, the privileges are granted sequentially. Prior to acquiring a new privilege, the client application shall release the previously acquired security context, if any exists, by calling the BSI's function gscBsiUtilReleaseContext () .

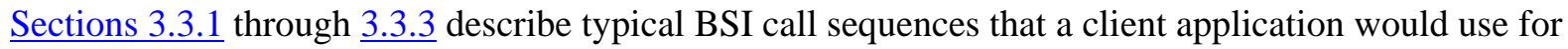
each of the three authentication mechanisms in order to acquire the context for the desired smart card service.

\subsubsection{PIN Verification}

For a PIN Verification known also as Card Holder Verification (CHV), the client application would make the following calls:

- Establish a logical connection with the card through a call to the BSI's function gscBsiUtilConnect ().

- Retrieve the ACRs for a desired card service through a call to either gscBsiGcGetContainerProperties () or gscBsiGetCryptoProperties (). These interface methods return the ACRs for all services available from the smart card (Sections 4.6.3 or 4.7.5, respectively). If PIN Verification is required for a particular service (e.g., gscBsiGcReadValue () or gscBsipkiCompute ()), the ACR returned in the GCacr or CRYPTOacr structure for this service must be BSI_ACR_PIN.

- Call gscBsiUtilAcquireContext () with the BSIAuthenticator structures required to satisfy the ACR for the desired smart card service. In this example, for PIN verification, the BSI Authenticator structure shall contain the PIN value in the authValue field and accessmethodType set to BSI_ACR_PIN.

- Access the desired smart card service through subsequent BSI calls.

- Call gscBsiutilReleaseContext () to release the security context.

\subsubsection{External Authentication}

A typical BSI sequence of calls for an External Authentication:

- Establish a logical connection with the card through a call to gscBsiutilConnect ().

- Retrieve the ACRs for a desired card service provider through a call to either gscBsiGcGetContainerProperties () or gscBsiGetCryptoProperties (). These interface methods return the ACRs for all services available from the smart card (Section 4.6.3 or Section 4.7.5 respectively). If External Authentication is required for a particular service (e.g., gscBsigcReadValue () or gscBsipkiCompute ()), the ACR returned in the GCacr or CRYPTOacr structure for this service must be BSI_ACR_XAUTH . 
- Call gscBsigetChallenge () to retrieve a random challenge from the smart card. The random challenge is retained by the smart card for use in the subsequent verification step of the External Authentication protocol. The client application calculates a cryptogram by encrypting the random challenge using a symmetric External Authentication key. The client application may need to examine the keyIDOrReference member of the appropriate ACR returned in GCacr or CRYPTOacr to determine which External Authentication key it should use to encrypt the random challenge.

- The client application calls the BSI's gscBsiUtilAcquireContext () function passing the cryptogram computed in the previous step.

- The smart card decrypts the Authenticator using its External Authentication key, and verifies that the resulting plaintext value matches the original random challenge value.

- Access the desired smart card service through subsequent BSI calls.

- Call gscBsiutilReleaseContext () to release the security context.

\subsubsection{Secure Messaging}

Secure messaging involves the establishment of a secure channel between the client application and the smart card at the APDU level. The BSI provides a pass-through call that allows a client application to establish a direct APDU level secure channel with a card in accordance with the Global Platform [GLOB] or ISO 7816-4 [ISO4] 


\section{Basic Services Interface}

\subsection{Overview}

An SPM must provide a BSI. Client applications communicate with the SPM through this interface. The SPS component of the SPM is directly responsible for implementing the BSI.

This chapter defines the BSI services, using notation similar to Interface Definition Language (IDL) which is referred to as pseudo IDL throughout this document. The set of services consists of 23 functions grouped into three functional modules as follows:

A Smart Card Utility Provider Module:

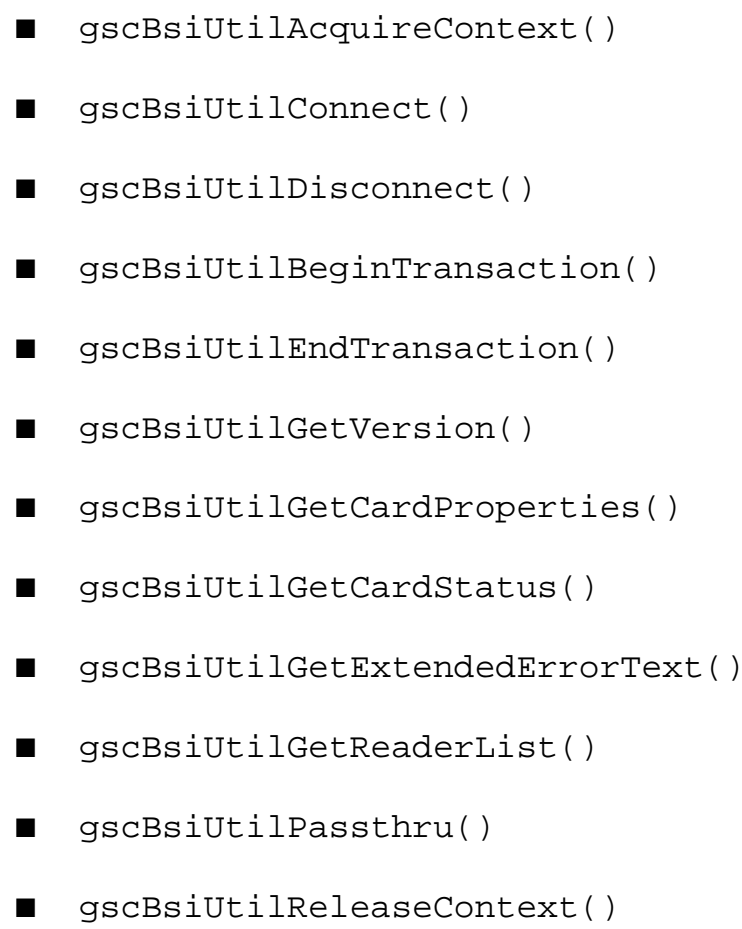

A Smart Card Generic Container Provider Module:

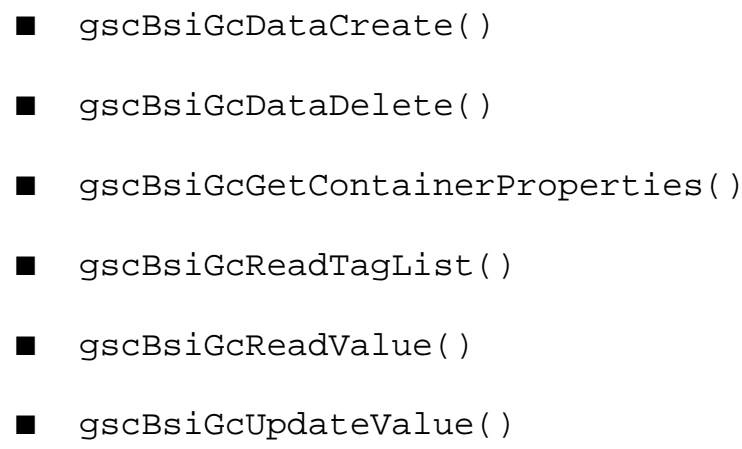

A Smart Card Cryptographic Provider Module:

- gscBsiGetChallenge() 
- gscBsiskiInternalAuthenticate()

- gscBsipkiCompute ()

- gscBsipkiGetCertificate()

- gscBsiGetCryptoproperties()

All SPM implementations must provide the full set of 23 functions as specified in this chapter. Based on the capabilities available, a given function call may return a BSI_NO_CARDSERVICE Or BSI_NO SPSSERVICE error message in case the SPM does not provide the requested service. This error message may be returned by any BSI function that maps directly to a card-level operation, as follows:

- gscBsiutilGetCardProperties ()

- gscBsigcDataCreate()

- gscBsigcDataDelete()

- gscBsiGcGetContainerProperties()

- gscBsiGcReadTaglist ()

- gscBsigcReadValue()

- gscBsiGcUpdateValue()

- gscBsiGetChallenge()

- gscBsiskiInternalAuthenticate()

- gscBsipkiCompute ()

- gscBsipkiGetCertificate()

- gscBsiGetCryptoproperties()

Extensions to the BSI, in the form of an XSI (see Section 2.3), may be present in an implementation to allow additional functionality. The functions in an XSI shall not alter the specified behavior or semantics of the BSI functions in that implementation.

ACRs for each provider module are defined in Chapter 3, Table 3-2, Table 3-3, and Table 3-4. Section $\underline{4.4}$ defines BSI return codes and Section 4.5 defines 23 functions of the BSI, using pseudo IDL.

\subsection{Binary Data Encoding}

BSI functions accept or return binary data, such as cryptograms. However, some of the BSI services may pass or get some ASCII or ASCII hexadecimal formatted data depending on the usage. In this case, each of the services involved must explicitly mention this and which of its parameter(s) is/are impacted.

\subsection{Mandatory Cryptographic Algorithms}

The following cryptographic algorithms and associated algorithm identifiers are mandatory for all GSC smart cards. These algorithm ID values are used as parameters at the BSI level. 
- Algorithm Identifier "0x81": DES3-ECB, with a double length key-size, 16 bytes.

- Algorithm Identifier "OxA3": RSA_NO_PAD, the private key computation, Chinese Remainder.

- Algorithm Identifier "0x82": DES3-CBC, with a double length key-size, 16 bytes.

\subsection{BSI Return Codes}

Table 4-1 lists all possible errors that BSI functions could return. For each function description (Sections 4.5.3 to 4.7.5), return codes are listed in order of precedence, except for the successful return with BSI_OK.

Table 4-1: BSI Return Codes

\begin{tabular}{|c|c|c|}
\hline Label & $\begin{array}{l}\text { Return Code } \\
\text { Hexadecimal Value }\end{array}$ & Meaning \\
\hline BSI_OK & $0 \times 00$ & Execution completed successfully. \\
\hline BSI_ACCESS_DENIED & $0 \times 01$ & The applicable ACR was not fulfilled. \\
\hline BSI_ACR_NOT_AVAILABLE & $0 \times 02$ & The specified ACR is incorrect. \\
\hline BSI_BAD_AID & $0 \times 03$ & $\begin{array}{l}\text { The specified Application Identifiers (AID) does } \\
\text { not exist. }\end{array}$ \\
\hline BSI_BAD_ALGO_ID & $0 \times 04$ & $\begin{array}{l}\text { The specified cryptographic algorithm is not } \\
\text { available. }\end{array}$ \\
\hline BSI_BAD_AUTH & $0 \times 05$ & Invalid authentication data. \\
\hline BSI_BAD_HANDLE & $0 \times 06$ & The specified card handle is not available. \\
\hline BSI_BAD_PARAM & $0 \times 07$ & $\begin{array}{l}\text { One or more of the specified parameters is } \\
\text { incorrect. }\end{array}$ \\
\hline BSI_BAD_TAG & $0 \times 08$ & Invalid tag information. \\
\hline BSI_CARD_ABSENT & $0 \times 09$ & $\begin{array}{l}\text { The smart card associated with the specified card } \\
\text { handle is not present. }\end{array}$ \\
\hline BSI_CARD_REMOVED & $0 \times 0 A$ & $\begin{array}{l}\text { The smart card associated with the specified card } \\
\text { handle has been removed. }\end{array}$ \\
\hline BSI_NO_SPSSERVICE & $0 \times 0 B$ & The SPS does not provide the requested service. \\
\hline BSI_IO_ERROR & $0 \times 0 \mathrm{C}$ & $\begin{array}{l}\text { Error encountered during input/output of the } \\
\text { specified data. }\end{array}$ \\
\hline- & $0 \times 0 D$ & RFU \\
\hline BSI_INSUFFICIENT_BUFFER & $0 \times 0 E$ & $\begin{array}{l}\text { The buffer allocated by the calling application is } \\
\text { too small. }\end{array}$ \\
\hline BSI_NO_CARDSERVICE & $0 \times 0 F$ & $\begin{array}{l}\text { The smart card associated with the specified card } \\
\text { handle does not provide the requested service. }\end{array}$ \\
\hline BSI_NO_MORE_SPACE & $0 \times 10$ & $\begin{array}{l}\text { There is insufficient space in the selected } \\
\text { container to store the specified data. }\end{array}$ \\
\hline BSI_PIN_BLOCKED & $0 \times 11$ & The PIN is blocked. \\
\hline- & $0 \times 012$ & RFU \\
\hline BSI_TAG_EXISTS & $0 \times 13$ & $\begin{array}{l}\text { The tag specified for a create operation already } \\
\text { exists in the target container. }\end{array}$ \\
\hline BSI_TIMEOUT_ERROR & $0 \times 14$ & $\begin{array}{l}\text { A connection could not be established with the } \\
\text { smart card before the timeout value expired. }\end{array}$ \\
\hline
\end{tabular}




\begin{tabular}{|l|c|l|}
\multicolumn{1}{|c|}{ Label } & \multicolumn{1}{|c|}{$\begin{array}{c}\text { Return Code } \\
\text { Hexadecimal Value }\end{array}$} & \multicolumn{1}{|c|}{ Meaning } \\
\hline BSI_TERMINAL_AUTH & $0 \times 15$ & $\begin{array}{l}\text { The card reader has performed a successful } \\
\text { authentication exchange with the smart card. }\end{array}$ \\
\hline BSI_NO_TEXT_AVAILABLE & $0 \times 16$ & No extended error text is available. \\
\hline BSI_UNKNOWN_ERROR & $0 \times 17$ & $\begin{array}{l}\text { The requested operation has generated an } \\
\text { unspecified error. }\end{array}$ \\
\hline BSI_UNKNOWN_READER & $0 \times 18$ & The specified reader does not exist. \\
\hline BSI_SC_LOCKED & $0 \times 19$ & $\begin{array}{l}\text { The smart card associated with the specified card } \\
\text { handle is under the exclusive transaction of } \\
\text { another client application (see blocking mode in } \\
\text { Section 4.5.6) }\end{array}$ \\
\hline BSI_NOT_TRANSACTED & 0x20 & The current transaction has not ended. \\
\hline
\end{tabular}

\subsection{Smart Card Utility Provider Module Interface Definition}

Section 4.5.1 presents the pseudo IDL used to define the 23 functions of the BSI services.

\subsubsection{Pseudo IDL Definition}

Using a modified Backus-Naur notation, a definition for the pseudo IDL is presented as follows:

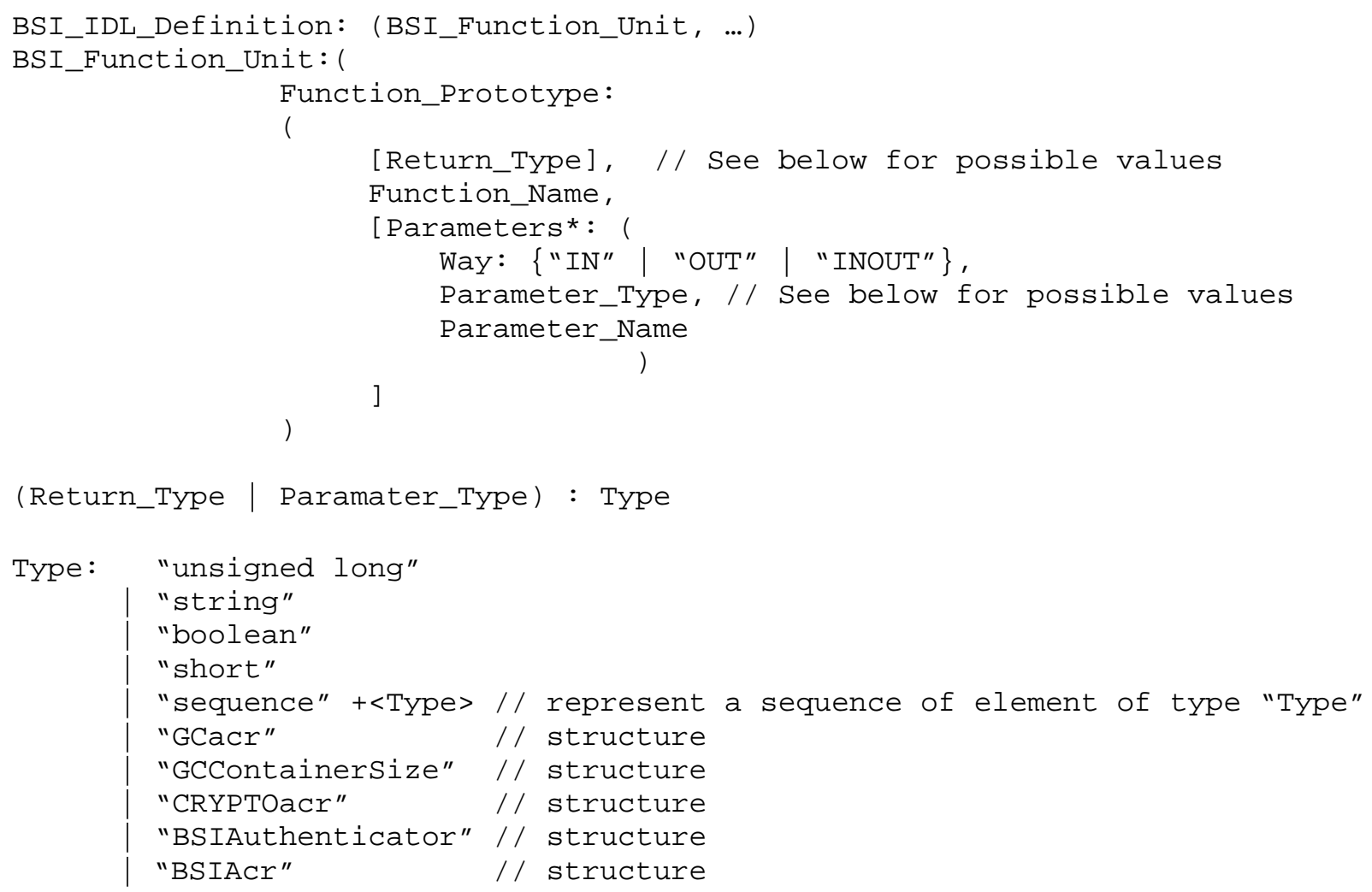

The types GCacr, GCContainerSize, CRYPTOacr and BSIAuthenticator are structure. The definition of a structure is as follows:

Struct_Definition: (Struct_Definition, ...) 


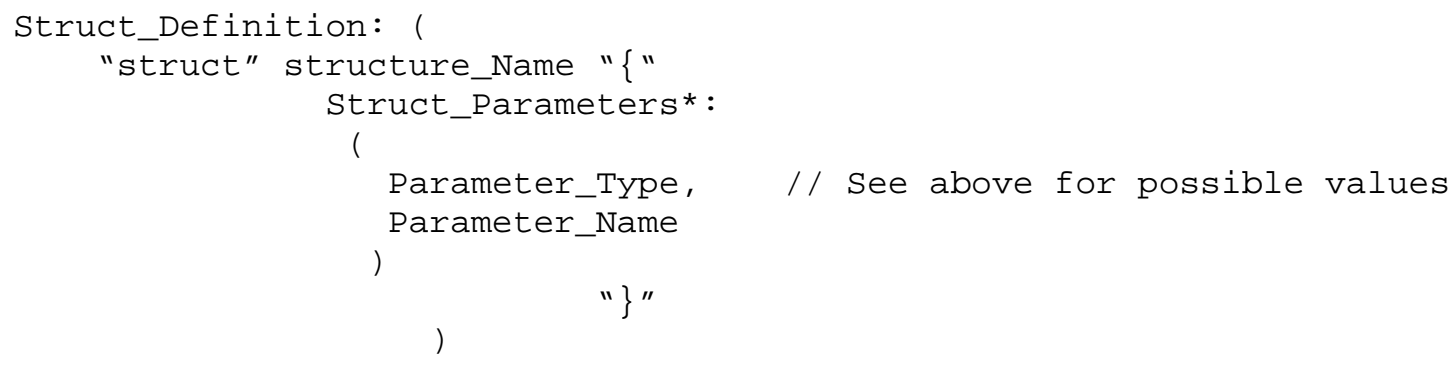

\subsubsection{Rules}

A description of the symbols used is in Table 4-2.

Table 4-2: Description of Symbols

\begin{tabular}{|l|l|}
\hline \multicolumn{1}{|c|}{ Symbol } & \multicolumn{1}{c|}{ Meaning } \\
\hline$:$ & is composed of \\
\hline[] & optional element \\
\hline() & includes or included in \\
\hline, & separates elements \\
\hline$\ldots$ & element repeats unspecified number of times \\
\hline\{\} & choose one from list \\
\hline$I$ & $\begin{array}{l}\text { or, indicates choice of possibilities for element } \\
\text { value }\end{array}$ \\
\hline+ & element is combined with preceding element \\
\hline$/ /$ & remainder of line contains comments \\
\hline '"' & contains a value \\
\hline$*$ & number of elements is zero or several \\
\hline
\end{tabular}

Tables 4-3 and 4-4 are the pseudo IDL to Java and pseudo IDL to C mappings for the different types specified above.

Table 4-3: Mapping Pseudo IDL to Java

\begin{tabular}{|l|l|}
\hline \multicolumn{1}{|c|}{ IDL type } & \multicolumn{1}{c|}{ Java type } \\
\hline unsigned long & int \\
\hline String & $\begin{array}{l}\text { byte[] or Java.lang.String (depending on the format : binary, } \\
\text { ASCll or ASCIl hex.) }\end{array}$ \\
\hline Boolean & boolean \\
\hline octet (unsigned 8 bits type) & short \\
\hline sequence $+<$ Type $>$ & $<$ Type $>$ [] or Vector of Type \\
\hline Gcacr & class Gcacr \\
\hline GCContainerSize & class GCContainerSize \\
\hline CRYPTOacr & class CRYPTOacr \\
\hline BSIAcr & Class BSIAcr \\
\hline
\end{tabular}


Table 4-4: Mapping Pseudo IDL to C

\begin{tabular}{|l|l|}
\hline \multicolumn{1}{|c|}{ IDL type } & \multicolumn{1}{c|}{ C type } \\
\hline unsigned long & unsigned long \\
\hline String & unsigned char * \\
\hline Boolean & boolean \\
\hline octet (unsigned 8 bits type) & unsigned char \\
\hline sequence + <Type $>$ & $<$ Type $>[$ (for byte see below) \\
\hline sequence<byte $>$ & unsigned char * \\
\hline Gcacr & struct Gcacr \\
\hline Gctag & unsigned char \\
\hline GCContainerSize & struct GCContainerSize \\
\hline CRYPTOacr & struct CRYPTOacr \\
\hline BSIAcr & struct BSIAcr \\
\hline String (with $n$ characters max, null terminated) & char[n] \\
\hline
\end{tabular}




\subsection{3 gscBsiUtilAcquireContext()}

Purpose:

Prototype:

Parameters: hCard:

AID:

strctAuthenticator:

authNb :
This function shall establish a session with a target container on the smart card by submitting the appropriate Authenticator in the BSIAuthenticator structure. For ACRs requiring external authentication (XAUTH), the authValue field of the BSIAuthenticator structure must contain a cryptogram calculated by encrypting a random challenge from gscBsiGetChallenge (). In cases where the card acceptance device authenticates the smart card, this function returns a BSI_TERMINAL_AUTH return code and the cryptogram is ignored.

For ACRs that require chained authentication such as BSI_ACR_PIN_AND_XAUTH, the calling application passes in the required authenticators in multiple BSIAuthenticator structures. In this example the calling application passes a PIN and the appropriate External Authentication cryptogram in two BSIAuthenticator structures. The client application must set the accessMethodType field of each BSIAuthenticator structure to match the type of authenticator contained in the structure. To satisfy an ACR of BSI_ACR_PIN_AND_XAUTH, the application would construct a sequence of two BSIAuthenticators: one containing a PIN and one containing an External Authentication cryptogram. The BSIAuthenticator structure containing the PIN would have an accessMethodType of BSI_AM_PIN, and the BSIAuthenticator structure containing the External Authentication cryptogram would have an accessMethodType of BSI_AM_XAUTH .

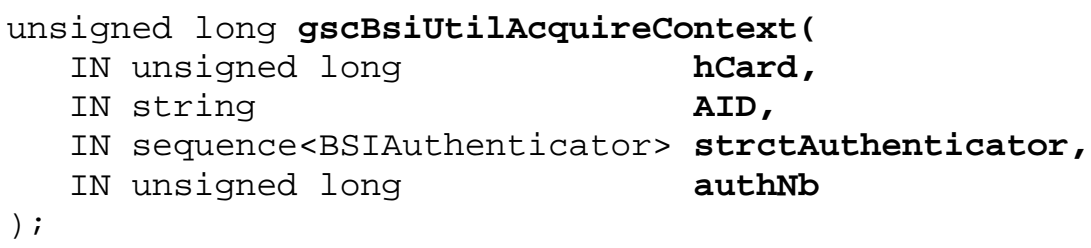
ASCII hexadecimal format.

Card connection handle from gscBsiutilconnect ().

Target container AID value. The parameter shall be in A sequence of structures containing the authenticator(s) specified by the ACR required to access a value in the container. The required list of authenticators is returned by gscBsiGcGetContainerProperties (). The calling application is responsible for allocating this structure.

Number of authenticator structures contained in strctAuthenticator.

The BSIAuthenticator structure is defined as follows:

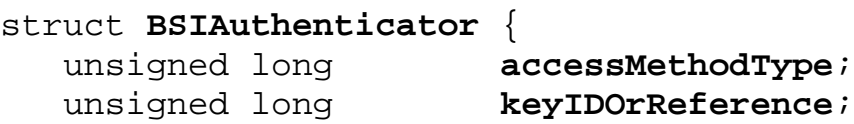


sequence<byte> authvalue;

\} ;

Variables associated with the BSIAuthenticator structure:

accessMethodType :

keyIDOrReference:

authvalue:

BSI_OK

BSI_BAD_HANDLE

BSI_BAD_AID

BSI_ACR_NOT_AVAILABLE

BSI_BAD_AUTH

BSI_CARD_REMOVED

BSI_PIN_BLOCKED

BSI_UNKNOWN_ERROR

BSI_TERMINAL_AUTH

BSI_BAD_PARAM

BSI_SC_LOCKED
Access Method Type (see Table 3-1 in Section 3.1). This function does not support secure channel and will return a BSI_BAD_PARAM if this field is set to one of the secure channel authentication methods.

Key identifier or reference of the authenticator. This is used to distinguish between multiple authenticators with the same Access Method Type.

Authenticator, can be an external authentication cryptogram or PIN. If the authenticator value is NULL, then the SPS is in charge of gathering authentication information and authenticating to the card. 


\subsection{4 gscBsiUtilConnect()}

Purpose: $\quad$ Establish a logical connection with the smart card in a specified reader. BSI_TIMEOUT_ERROR will be returned if a connection cannot be established within a specified time. The timeout value is implementation dependent.

Prototype:

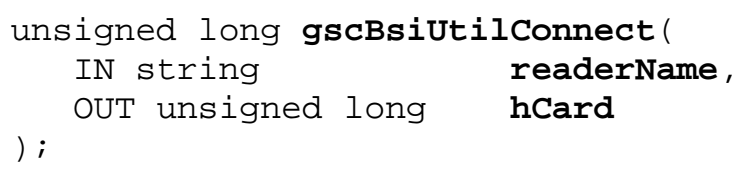

Parameters: hCard: $\quad$ Card connection handle.

readerName: Name of the reader that the smart card is inserted into. If this field is a NULL pointer, the SPS shall attempt to connect to the smart card in the first available reader, as returned by a call to the BSI's function

gscBsiutilgetReaderList () . The reader name string shall be stored as ASCII encoded String. (See Section 4.2)

$\begin{array}{ll}\text { Return Codes: } & \text { BSI_OK } \\ & \text { BSI_BAD_PARAM } \\ & \text { BSI_UNKNOWN_READER } \\ & \text { BSI_CARD_ABSENT } \\ & \text { BSI_TIMEOUT_ERROR } \\ & \text { BSI_UNKNOWN_ERROR }\end{array}$


NIST IR 6887 - 2003 EDITION, GSC-IS VERSION 2.1

\subsection{5 gscBsiUtilDisconnect()}

Purpose: $\quad$ Terminate a logical connection to a smart card.

Prototype: $\quad$ unsigned long gscBsiutildisconnect (

IN unsigned long hCard

$$
\text { ) ; }
$$

Parameters: hCard: Card connection handle from gscBsiutilconnect ().

Return Codes: $\quad$ BSI_OK

BSI_BAD_HANDLE

BSI_CARD_REMOVED

BSI_UNKNOWN_ERROR 


\subsection{6 gscBsiUtilBeginTransaction()}

Purpose:

Prototype:

Parameters: hCard:

blType:

$\begin{array}{ll}\text { Return Codes: } & \text { BSI_OK } \\ & \text { BSI_BAD_HANDLE } \\ & \text { BSI_UNKNOWN_ERROR } \\ & \text { BSI_SC_LOCKED } \\ & \text { BSI_NOT_TRANSACTED } \\ & \text { BSI_NO_SPSSERVICE }\end{array}$

This function starts an exclusive transaction with the smart card referenced by hCard. When the transaction starts, all other applications are precluded from accessing the smart card while the transaction is in progress. Two types of calls can be made: a blocking transaction call and a non-blocking transaction call, with a boolean type parameter identifying which mode is called. In the non-blocking mode, the call will return immediately if another client has an active transaction lock. The returned error code will be BSI_SC_LOCKED. In the blocking mode, the call will wait indefinitely for any active transaction locks to be released. A transaction must be completed by a call to gscBsiutilendTransaction() .

For single-threaded BSI implementations, it can be assumed that each application will be associated with a separate process. The same process that starts a transaction must also complete the transaction. For multi-threaded BSI implementations, it can be assumed that each application will be associated with a separate thread and/or process. The same thread that starts a transaction must also complete the transaction.

If this function is called by a thread that has already called gscBsiutilBeginTransaction() but has not yet called gscBsiUtilendTransaction(), it will return the error BSI_NOT_TRANSACTED.

If the SPS (Service Provider Software) does not support transaction locking, it should return the error code BSI_NO_SPSSERVICE in response to a call to gscBsiutilBeginTransaction().

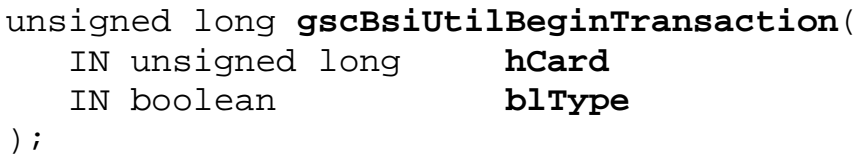

Card communication handle returned from gscBsiutilconnect ()

Boolean specifying the type of transaction call ( bl Type set to "true" in blocking mode. bl Type set to "false" in non-blocking mode). 


\subsection{7 gscBsiUtilEndTransaction()}

Purpose: $\quad$ This function ends a previously started transaction, allowing other blocked applications to begin or resume interactions with the card.

If this function is called by a thread that has not yet called gscBsiutilBeginTransaction(), it will return the error BSI_NOT_TRANSACTED.

If the SPS does not support transaction locking, it should return the error code BSI_NO_SPSSERVICE in response to a call to gscBsiUtilEndTransaction().

Prototype: unsigned long gscBsiutilendTransaction(
IN unsigned long hCard

Parameters: hCard: $\quad$ Card communication handle returned from gscBsiutilConnect ().

Return Codes: BSI_OK

BSI_BAD_HANDLE

BSI_UNKNOWN_ERROR

BSI_NOT_TRANSACTED

BSI_NO_SPSSERVICE 


\subsection{8 gscBsiUtilGetVersion()}

Purpose: Returns the BSI implementation version.

Prototype: unsigned long gscBsiutilgetversion( INOUT string version

) ;

Parameters: version: The BSI and SPS version formatted as "major,minor,revision,build_number". The value for an SPS conformant with this version of the GSC-IS is " $2,1,0,<$ build number>". The build number field is vendor/implementation dependent. The version name string shall be stored as ASCII encoded String. (See Section 4.2)

Return Codes: $\quad$ BSI_OK

BSI_INSUFFICIENT_BUFFER

BSI_UNKNOWN_ERROR 


\subsection{9 gscBsiUtilGetCardProperties()}

Purpose: $\quad$ Retrieves Card Capability Container ID and capability information for the smart card.

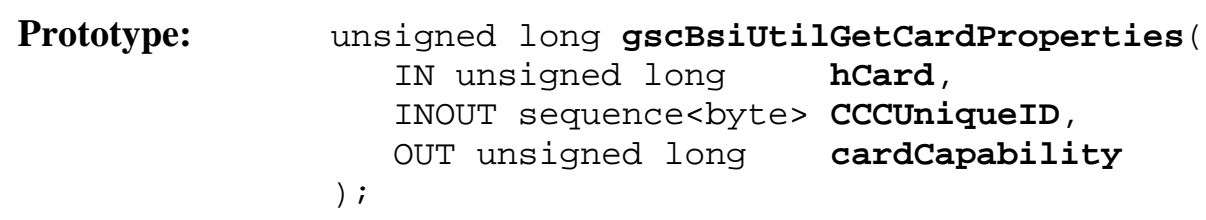

Parameters: hCard: Card connection handle from gscBsiutilconnect () .

CCCUniqueID: $\quad$ Buffer for the Card Capability Container ID.

cardCapability: $\quad$ Bit mask value defining the providers supported by the smart card. The bit masks represent the Generic Container Data Model, the Symmetric Key Interface, and the Public Key Interface providers respectively:

$\begin{array}{ll}\text { \#define BSI_GCCDM } & \text { 0x00000001 } \\ \text { \#define BSI_SKI } & 0 \times 00000002 \\ \text { \#define BSI_PKI } & \text { 0x00000004 }\end{array}$

Return Codes: $\quad$ BSI_OK

BSI_BAD_HANDLE

BSI_CARD_REMOVED

BSI_INSUFFICIENT_BUFFER

BSI_NO_CARDSERVICE

BSI_UNKNOWN_ERROR

BSI_SC_LOCKED 


\subsubsection{0 gscBsiUtilGetCardStatus()}

Purpose: $\quad$ Checks whether a given card handle is associated with a smart card that is inserted into a powered up reader.

Prototype: $\quad$ unsigned long gscBsiutilgetCardStatus (

IN unsigned long hCard

) ;

Parameters: hCard: $\quad$ Card connection handle from gscBsiutilconnect ().

Return Codes: $\quad$ BSI_OK

BSI_BAD_HANDLE

BSI_CARD_REMOVED

BSI_UNKNOWN_ERROR 


\subsubsection{1 gscBsiUtilGetExtendedErrorText()}

Purpose: $\quad$ When a BSI function call returns an error, an application can make a subsequent call gscBsiUtilGetExtendedErrorText to receive additional error information from the card reader driver layer, if available. Since the GSC-IS architecture accommodates different card reader driver layers, the error text information will be dependent on the card reader driver layer used in a particular implementation. This function must be called immediately after the error has occurred.

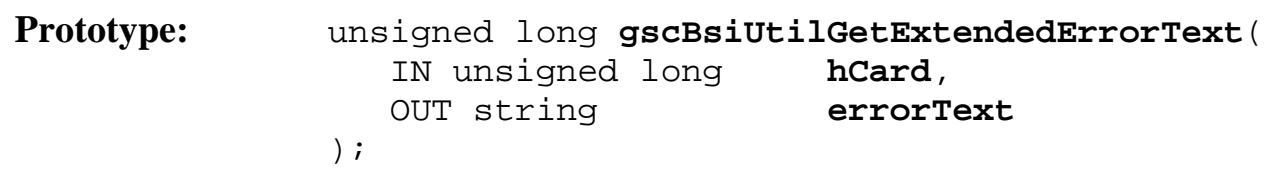

Parameters: hCard: $\quad$ Card connection handle from gscBsiutilconnect ().

errorText: $\quad$ A fixed length buffer containing an implementation specific error text string. The text string has a maximum length of 255 characters. The calling application must allocate a buffer of 255 bytes. If an extended error text string is not available, this function returns a NULL string and the return code BSI_NO_TEXT_AVAILABLE. The error text string shall be stored as ASCII encoded String. (See Section 4.2)

$\begin{array}{ll}\text { Return Codes: } & \text { BSI_OK } \\ & \text { BSI_BAD_HANDLE } \\ & \text { BSI_NO_TEXT_AVAILABLE } \\ & \text { BSI_UNKNOWN_ERROR }\end{array}$




\subsubsection{2 gscBsiUtilGetReaderList()}

Purpose: $\quad$ Retrieves the list of available readers.

Prototype: unsigned long gscBsiutilgetReaderlist ( INOUT sequence<string readerList

) ;

Parameters: readerList: $\quad$ Reader list buffer. The reader list is returned as a multistring. The list of available readers shall be stored as ASCII encoded String. (See Section 4.2)

Return Codes: $\quad$ BSI_OK

BSI_INSUFFICIENT_BUFFER

BSI_UNKNOWN_ERROR 


\subsubsection{3 gscBsiUtilPassthru()}

Purpose: $\quad$ Allows a client application to send a "raw" ISO 7816-4 [ISO4] APDU through the BSI directly to the smart card and receive the APDU-level response.

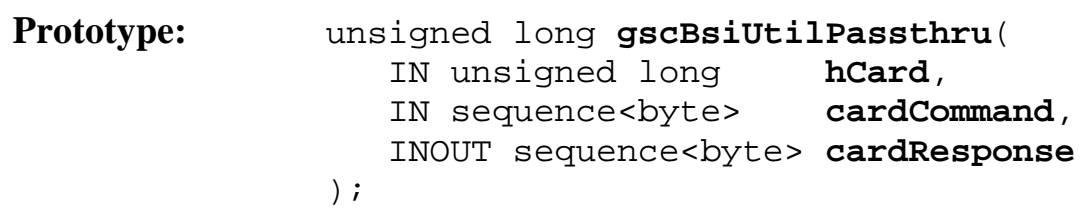

Parameters: hCard: $\quad$ Card connection handle from gscBsiutilConnect ().

cardCommand: $\quad$ The APDU to be sent to the smart card. That parameter must be in ASCII hexadecimal format.

cardResponse: $\quad$ Pre-allocated buffer for the APDU response from the smart card. The response must include the status bytes SW1 and SW2 returned by the smart card. If the size of the buffer is insufficient, the SPS shall return truncated response data and the return code

BSI_INSUFFICIENT_BUFFER. That parameter must be in ASCII hexadecimal format.

$\begin{array}{ll}\text { Return Codes: } & \text { BSI_OK } \\ & \text { BSI_BAD_HANDLE } \\ & \text { BSI_BAD_PARAM } \\ & \text { BSI_INSUFFICIENT_BUFFER } \\ & \text { BSI_CARD_REMOVED } \\ & \text { BSI_UNKNOWN_ERROR } \\ & \text { BSI_SC_LOCKED }\end{array}$




\subsubsection{4 gscBsiUtilReleaseContext()}

Purpose: $\quad$ Terminate a session with the target container on the smart card.

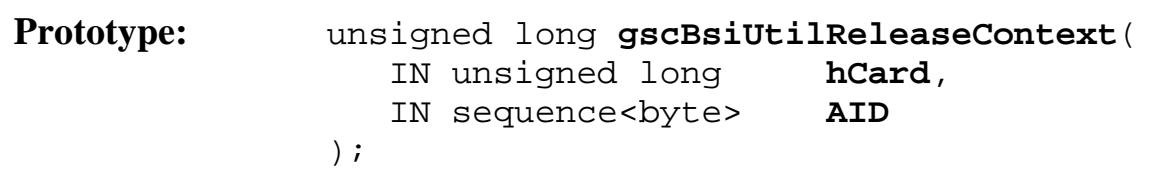

Parameters: hCard: $\quad$ Card connection handle from gscBsiutilconnect ().

AID :

Target container AID value. The AID shall be stored as an ASCII hexadecimal string.

$\begin{array}{ll}\text { Return Codes: } & \text { BSI_OK } \\ & \text { BSI_BAD_HANDLE } \\ & \text { BSI_BAD_AID } \\ & \text { BSI_CARD_REMOVED } \\ & \text { BSI_UNKNOWN_ERROR } \\ & \text { BSI_SC_LOCKED }\end{array}$




\subsection{Smart Card Generic Container Provider Module Interface Definition}

\subsection{1 gscBsiGcDataCreate()}

Purpose: $\quad$ Create a new data item in $\{$ Tag, Length, Value $\}$ format in the selected container.

\begin{tabular}{lll} 
Prototype: & \multicolumn{3}{c}{ unsigned long gscBsiGcDataCreate ( } \\
IN unsigned long & hCard, \\
IN string & AID, \\
IN octet & tag, \\
& IN sequence<byte> & value \\
); &
\end{tabular}

\begin{tabular}{|c|c|c|}
\hline \multirow[t]{3}{*}{ Parameters: } & hCard: & Card connection handle from gscBsiutilconnect () \\
\hline & AID : & $\begin{array}{l}\text { Target container AID value. The parameter shall be in } \\
\text { ASCII hexadecimal format. }\end{array}$ \\
\hline & tag: & Tag of data item to store. \\
\hline & value: & Data value to store. \\
\hline
\end{tabular}

Return Codes: $\quad$ BSI_OK

BSI_BAD_HANDLE

BSI_BAD_AID

BSI_BAD_PARAM

BSI_CARD_REMOVED

BSI_NO_CARDSERVICE

BSI_ACCESS_DENIED

BSI_NO_MORE_SPACE

BSI_TAG_EXISTS

BSI_IO_ERROR

BSI_UNKNOWN_ERROR

BSI_SC_LOCKED 


\subsection{2 gscBsiGcDataDelete()}

Purpose: $\quad$ Delete the data item associated with the tag value in the specified container.

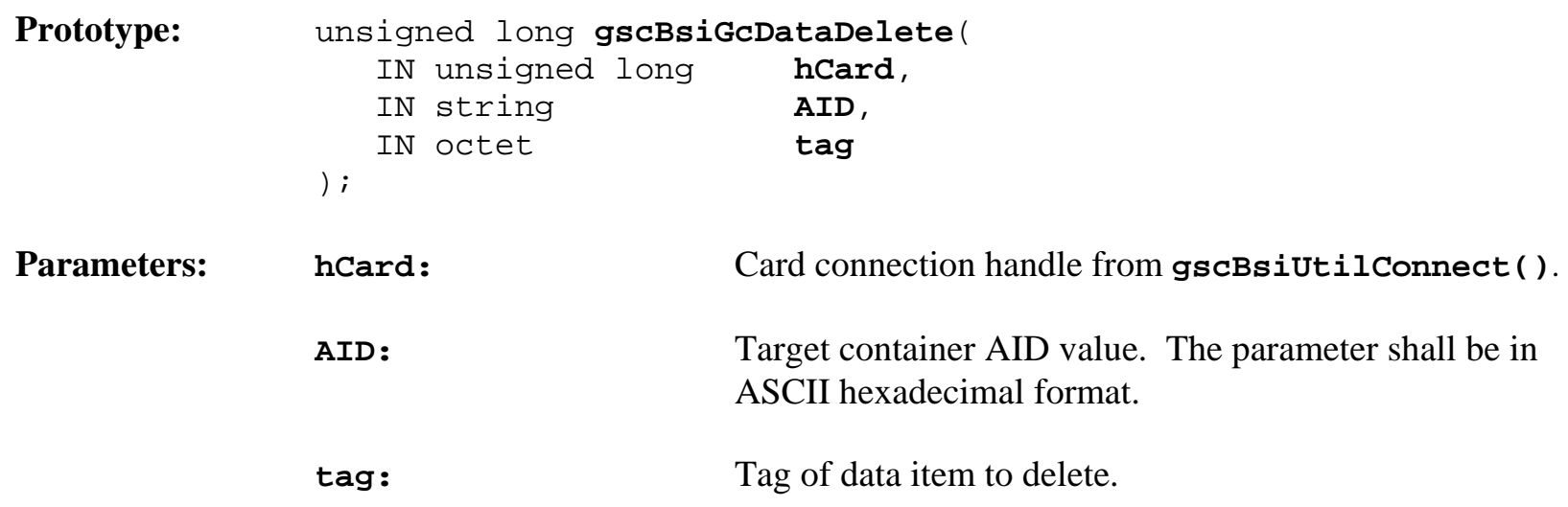

Return Codes: $\quad$ BSI_OK
BSI_BAD_HANDLE
BSI_BAD_AID
BSI_BAD_TAG
BSI_CARD_REMOVED
BSI_NO_CARDSERVICE
BSI_SC_LOCKED
BSI_ACCESS_DENIED
BSI_IO_ERROR
BSI_UNKNOWN_ERROR 
\}

containerVersion: Version of the container. The format of this value is application dependent. In cases where the smart card cannot return a container version, this byte sequence will be empty.

$\begin{array}{ll}\text { Return Codes: } & \text { BSI_OK } \\ & \text { BSI_BAD_HANDLE } \\ & \text { BSI_BAD_AID } \\ & \text { BSI_CARD_REMOVED } \\ & \text { BSI_SC_LOCKED } \\ & \text { BSI_NO_CARDSERVICE } \\ & \text { BSI_UNKNOWN_ERROR }\end{array}$




\subsection{4 gscBsiGcReadTagList()}

Purpose: $\quad$ Return the list of tags in the selected container.

$\begin{array}{lll}\text { Prototype: } & \text { unsigned long gscBsiGcReadTagList ( } \\ & \text { IN unsigned long } & \text { hCard, } \\ \text { IN string } & \text { AID, } \\ & \text { INOUT sequence<octet> } & \text { tagArray } \\ \text { ); } & \end{array}$

Parameters: hCard: $\quad$ Card connection handle from gscBsiutilconnect ().

AID: $\quad$ Target container AID value. The parameter shall be in ASCII hexadecimal format.

tagArray: An array containing the list of tags for the selected container.

$\begin{array}{ll}\text { Return Codes: } & \text { BSI_OK } \\ & \text { BSI_BAD_HANDLE } \\ & \text { BSI_BAD_AID } \\ & \text { BSI_CARD_REMOVED } \\ & \text { BSI_SC_LOCKED } \\ & \text { BSI_NO_CARDSERVICE } \\ & \text { BSI_ACCESS_DENIED } \\ & \text { BSI_INSUFFICIENT_BUFFER } \\ & \text { BSI_UNKNOWN_ERROR }\end{array}$




\subsection{5 gscBsiGcReadValue()}

Purpose: $\quad$ Returns the Value associated with the specified Tag.

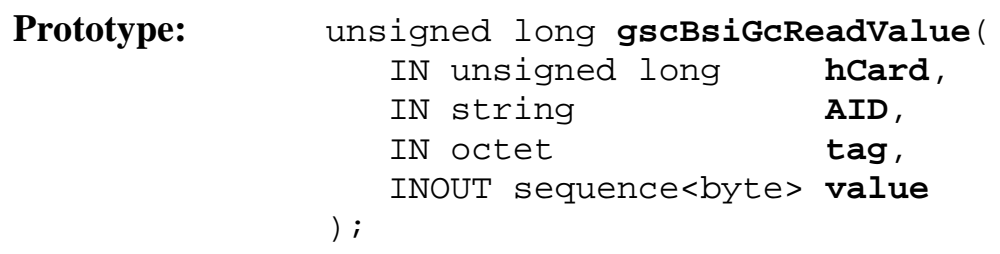

Parameters: hCard: $\quad$ Card connection handle from gscBsiutilConnect ().

AID: $\quad$ Target container AID value. The parameter shall be in ASCII hexadecimal format.

tag: $\quad$ Tag value of data item to read.

value: $\quad$ Value associated with the specified tag. The client application must allocate the buffer.

$\begin{array}{ll}\text { Return Codes: } & \text { BSI_OK } \\ & \text { BSI_BAD_HANDLE } \\ & \text { BSI_BAD_AID } \\ & \text { BSI_BAD_TAG } \\ & \text { BSI_CARD_REMOVED } \\ & \text { BSI_SC_LOCKED } \\ & \text { BSI_NO_CARDSERVICE } \\ & \text { BSI_ACCESS_DENIED } \\ & \text { BSI_INSUFFICIENT_BUFFER } \\ & \text { BSI_IO_ERROR } \\ & \text { BSI_UNKNOWN_ERROR }\end{array}$




\subsection{6 gscBsiGcUpdateValue()}

Purpose: $\quad$ Updates the Value associated with the specified Tag.

\begin{tabular}{|c|c|c|c|}
\hline \multirow[t]{5}{*}{ Prototype: } & \multicolumn{3}{|c|}{ unsigned long gscBsiGcUpdatevalue } \\
\hline & IN & unsigned long & hCard, \\
\hline & IN & string & AID, \\
\hline & IN & octet & tag, \\
\hline & IN & sequence $<$ byte $>$ & value \\
\hline
\end{tabular}

\begin{tabular}{|c|c|c|}
\hline \multirow[t]{3}{*}{ Parameters: } & hCard: & Card connection handle from gscBsiutilconnect () \\
\hline & AID : & $\begin{array}{l}\text { Target container AID value. The parameter shall be in } \\
\text { ASCII hexadecimal format. }\end{array}$ \\
\hline & tag: & Tag of data item to update. \\
\hline & value: & New Value of the data item. \\
\hline
\end{tabular}

Return Codes: $\quad$ BSI_OK

BSI_BAD_HANDLE

BSI_BAD_AID

BSI_BAD_PARAM

BSI_BAD_TAG

BSI_CARD_REMOVED

BSI_SC_LOCKED

BSI_NO_CARDSERVICE

BSI_ACCESS_DENIED

BSI_NO_MORE_SPACE

BSI_IO_ERROR

BSI_UNKNOWN_ERROR 


\subsection{Smart Card Cryptographic Provider Module Interface Definition}

\subsection{1 gscBsiGetChallenge()}

Purpose: $\quad$ Retrieves a randomly generated challenge from the smart card as the first step of a challenge-response authentication protocol between the client application and the smart card. The client subsequently encrypts the challenge using a symmetric key and returns the encrypted random challenge to the smart card through a call to gscBsiutilAcquireContext () in the authValue field of a BSIAuthenticator structure.

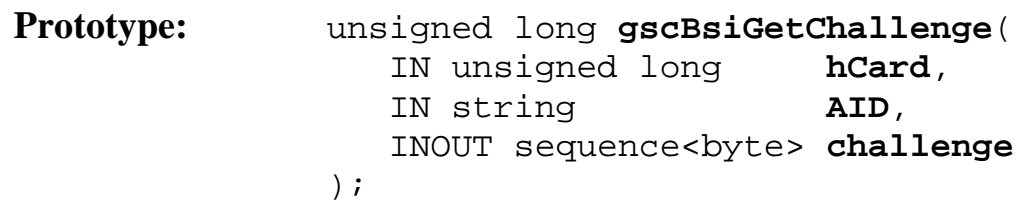

Parameters: hCard: $\quad$ Card connection handle from gscBsiutilconnect (). AID: $\quad$ Target container AID value. The parameter shall be in ASCII hexadecimal format.

challenge: $\quad$ Random challenge returned from the smart card.

$\begin{array}{ll}\text { Return Codes: } & \text { BSI_OK } \\ & \text { BSI_BAD_HANDLE } \\ & \text { BSI_BAD_AID } \\ & \text { BSI_CARD_REMOVED } \\ & \text { BSI_SC_LOCKED } \\ & \text { BSI_NO_CARDSERVICE } \\ & \text { BSI_INSUFFICIENT_BUFFER } \\ & \text { BSI_UNKNOWN_ERROR }\end{array}$




\subsection{2 gscBsiSkilnternalAuthenticate()}

Purpose: Computes a symmetric key cryptogram in response to a challenge. In cases where the card reader authenticates the smart card, this function does not return a cryptogram. In these cases a BSI_TERMINAL_AUTH will be returned if the card reader successfully authenticates the smart card. BSI_ACCESS_DENIED is returned if the card reader fails to authenticate the smart card.

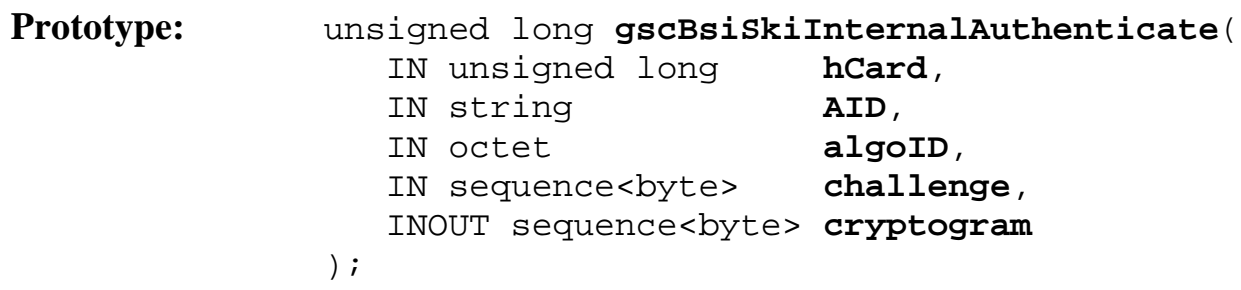

\section{Parameters: hCard:}

AID :

algoID:

challenge:

cryptogram :
Card connection handle from gscBsiutilconnect () .

SKI provider module AID value. The parameter shall be in ASCII hexadecimal format.

Identifies the cryptographic algorithm that the smart card must use to encrypt the challenge. All conformant implementations shall, at a minimum, support DES3ECB (Algorithm Identifier 0x81) and DES3-CBC (Algorithm Identifier 0x82). Implementations may optionally support other cryptographic algorithms.

Challenge generated by the client application and submitted to the smart card.

The cryptogram computed by the smart card.

\footnotetext{
Return Codes: $\quad$ BSI_OK

BSI_BAD_HANDLE

BSI_BAD_AID

BSI_BAD_PARAM

BSI_BAD_ALGO_ID

BSI_CARD_REMOVED

BSI_SC_LOCKED

BSI_NO_CARDSERVICE

BSI_ACCESS_DENIED

BSI_TERMINAL_AUTH

BSI_INSUFFICIENT_BUFFER

BSI_UNKNOWN_ERROR
} 


\subsection{3 gscBsiPkiCompute()}

Purpose: $\quad$ Performs a private key computation on the message digest using the private key associated with the specified AID.

Prototype:

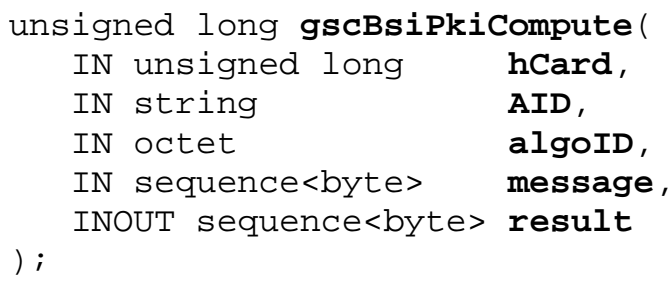

Parameters: hCard: $\quad$ Card connection handle from gscBsiutilconnect () .

AID :

algoID :

message :

result :
PKI provider module AID value. The parameter shall be in ASCII hexadecimal format.

Identifies the cryptographic algorithm that will be used to generate the signature. All conformant implementations shall, at a minimum, support RSA_NO_PAD (Algorithm Identifier 0xA3). Implementations may optionally support other algorithms.

The message digest to be signed.

Buffer containing the signature.

Return Codes: $\quad$ BSI_OK

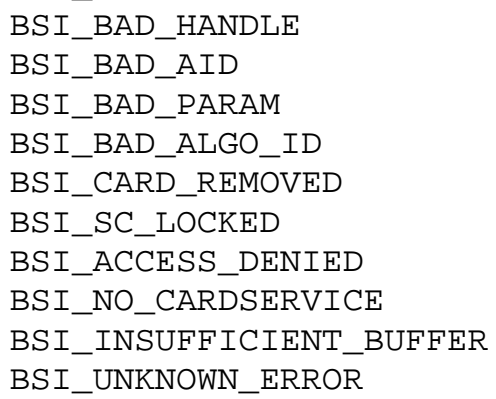




\subsection{4 gscBsiPkiGetCertificate()}

Purpose: Reads the certificate from the smart card.

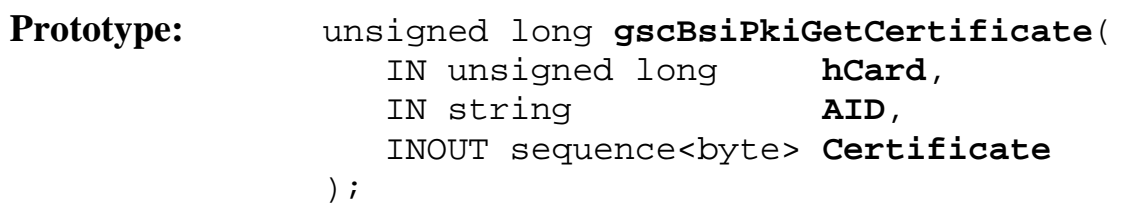

Parameters: hCard: $\quad$ Card connection handle from gscBsiutilconnect ().

AID: $\quad$ PKI provider module AID value. The parameter shall be in ASCII hexadecimal format.

certificate: $\quad$ Buffer containing the certificate.

$\begin{array}{ll}\text { Return Codes: } & \text { BSI_OK } \\ & \text { BSI_BAD_HANDLE } \\ & \text { BSI_BAD_AID } \\ & \text { BSI_CARD_REMOVED } \\ & \text { BSI_SC_LOCKED } \\ & \text { BSI_NO_CARDSERVICE } \\ & \text { BSI_ACCESS_DENIED } \\ & \text { BSI_IO_ERROR } \\ & \text { BSI_INSUFFICIENT_BUFFER } \\ \text { BSI_UNKNOWN_ERROR }\end{array}$




\subsection{5 gscBsiGetCryptoProperties()}

Purpose: $\quad$ Retrieves the Access Control Rules associated with the PKI provider module.

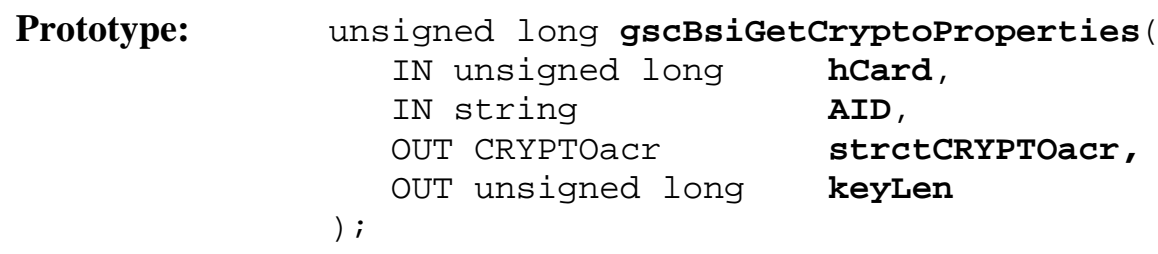

Parameters: hCard:

AID :

strctCRYPTOacr:
Card connection handle from gscBsiutilConnect () .

AID of the PKI provider. The parameter shall be in ASCII hexadecimal format.

Structure indicating access control conditions for all operations. The BSIAcr structure is defined in Section 4.6.3. The range of possible values for the members of this structure are defined in Table 3-2 (Section 3.1), and the allowable ACRs for each function in Table 3-4 (Section 3.2). keyIDOrReference contains the key identifier or reference for each access method contained in the ACR in order of appearance. authNb is the number of access methods logically combined in the ACR. ACRID is RFU and must be NULL (0x00) in this version. Note that the readValueACR member maps to the gscBsipkiGetCertificate() function.

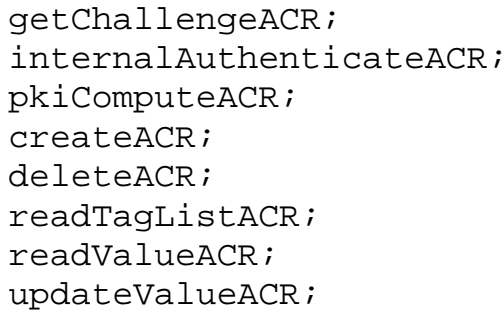

Length in bits of the private key managed by the PKI provider.
Return Codes: $\quad$ BSI_OK
BSI_BAD_HANDLE
BSI_BAD_AID
BSI_CARD_REMOVED
BSI_SC_LOCKED
BSI_NO_CARDSERVICE
BSI_UNKNOWN_ERROR


NIST IR 6887 - 2003 EDITION, GSC-IS VERSION 2.1

\section{THIS PAGE INTENTIONALLY LEFT BLANK.}




\section{Virtual Card Edge Interface}

The Virtual Card Edge Interface includes two sets of APDU commands: (1) an ISO 7816-4 [ISO4] and 7816-8 [ISO8] conformant GSC-IS APDU set for use in conformant file system cards, and (2) a set of VM APDUs for use in VM cards. The card edge also consists of the CCC, which is a file located on each conformant smart card, and the GSC-IS APDU mapping mechanism.

The GSC-IS ISO-conformant APDU set can be implemented directly by conformant cards (such as in a conformant file system card or as a VM card applet). It is expected that some file system smart cards may use native APDU instruction sets that will differ from the GSC-IS APDU set. In those cases, an SPS must modify the ADPU set such that it conforms to the smart card's native APDU set. This is done using the GSC-IS APDU mapping mechanism described in Section 5.2 and in Chapter 6.

Sections 5.1 through 5.3 describe the GSC-IS APDU set, overview information on the procedures for mapping this APDU set to smart card-specific APDU sets, and the APDUs for VM cards only. Chapter 6 provides details on the rules and procedures for APDU translations according to the CCC grammar.

\subsection{GSC-IS ISO Conformant APDUs}

Table 5-1 shows the GSC-IS APDU set for file system and VM cards. The APDUs are conformant with ISO 7816-4 [ISO4] and 7816-8 [ISO8], however some values have been defined for cryptogram lengths and cryptographic algorithm identifiers. Additional behavior for the APDUs would be described in a smart card's CCC tuples using the descriptor code mechanisms. Support for secure messaging is not provided in this APDU set; as described in Section 3.3.3, secure messaging is implemented via the gscBsiUtilpassthru () mechanism in accordance with the Global Platform [GLOB] or ISO 7816-4 [ISO4].

Table 5-1: GSC-IS APDU Set

\begin{tabular}{|l|l|}
\hline \multicolumn{1}{|c|}{ GSC-IS APDU Set } \\
\hline \multirow{4}{*}{$\begin{array}{c}\text { Generic File Access } \\
\text { APDUs }\end{array}$} & GET RESPONSE \\
\cline { 2 - 2 } & READ BINARY \\
\cline { 2 - 3 } & SELECT DF \\
\cline { 2 - 3 } & SELECT EF UNDER SELECTED DF \\
\cline { 2 - 3 } & SELECT FILE \\
\cline { 2 - 3 } & SELECT MASTER FILE (Root) \\
\cline { 2 - 3 } & UPDATE BINARY \\
\hline \multirow{5}{*}{ Access Control APDUs } & GET CHALLENGE \\
\cline { 2 - 3 } & INTERNAL AUTHENTICATE \\
\cline { 2 - 3 } & VERIFY \\
\hline
\end{tabular}




\begin{tabular}{|c|l|}
\hline \multicolumn{2}{|c|}{ GSC-IS APDU Set } \\
\hline \multirow{2}{*}{$\begin{array}{c}\text { Public Key Operations } \\
\text { APDUs }\end{array}$} & MANAGE SECURITY ENVIRONMENT \\
\cline { 2 - 2 } & PERFORM SECURITY OPERATION \\
\hline
\end{tabular}

The APDUs are divided into three categories: Generic File Access, Access Control, and Public Key Operations. The ADPU commands and responses are structured as follows:

Table 5-2: APDU Command and Response Structure

\begin{tabular}{|l|l|l|l|l|l|l|}
\hline \multicolumn{7}{|c|}{ Command APDU } \\
\hline CLA & INS & P1 & P2 & $L_{c}$ & Data Field & $L_{e}$ \\
\hline
\end{tabular}

\begin{tabular}{|l|c|c|}
\hline \multicolumn{3}{|c|}{ Response APDU } \\
\hline Response & SW1 & SW2 \\
\hline
\end{tabular}

The terms described in Table 5-3 are used throughout this section.

Table 5-3: APDU Command and Response Structure

\begin{tabular}{|l|l|}
\hline \multicolumn{1}{|c|}{ APDU Term } & \multicolumn{1}{c|}{ Description } \\
\hline CLA & Class byte \\
\hline Data Field & String of bytes sent in the data field of the command \\
\hline FC & $\begin{array}{l}\text { Function code, used in the CCC grammar to identify the } \\
\text { default APDU that is being mapped (see Chapter 6 for } \\
\text { detailed information) }\end{array}$ \\
\hline $\mathrm{L}_{c}$ & Number of bytes present in data field of the command \\
\hline $\mathrm{L}_{e}$ & $\begin{array}{l}\text { Maximum number of bytes expected in the data field of the } \\
\text { response to the command }\end{array}$ \\
\hline INS & $\begin{array}{l}\text { Instruction byte; ISO 7816 defines a set of common } \\
\text { commands, e.g., 'B0' is Read Binary }\end{array}$ \\
\hline P1-P2 & Instruction parameter 1 and 2 \\
\hline Response & String of bytes received in the data field of the response \\
\hline SW1 & $\begin{array}{l}\text { Command processing status, i.e., the return code from the } \\
\text { smart card }\end{array}$ \\
\hline SW2 & $\begin{array}{l}\text { Command processing qualifier, supplies further information on } \\
\text { SW1 }\end{array}$ \\
\hline
\end{tabular}

\subsubsection{Generic File Access APDUs}

The APDUs in Table 5-4 are used to perform basic file access functions. 
Table 5-4: Generic File Access APDUs

\begin{tabular}{|c|c|c|c|c|c|c|c|c|}
\hline \multicolumn{9}{|c|}{ Generic File Access APDUs } \\
\hline FC & Card Function & CLA & INS & P1 & P2 & $\mathrm{L}_{\mathrm{c}}$ & Data & $\mathbf{L}_{e}$ \\
\hline $0 \times 07$ & GET RESPONSE & $0 \times 00$ & $0 \times \mathrm{CO}$ & $0 \times 00$ & $0 \times 00$ & - & - & $\mathrm{L}_{\mathrm{e}}$ \\
\hline $0 \times 02$ & READ BINARY & $0 \times 00$ & $0 \times B 0$ & $\mathrm{Off} / \mathrm{H}$ & Off/L & - & - & $\mathrm{L}_{\mathrm{e}}$ \\
\hline $0 \times 01$ & SELECT DF & $0 \times 00$ & $0 \times A 4$ & $0 \times 01$ & $\begin{array}{l}0 \times 00 \\
\text { or } \\
0 \times 0 C\end{array}$ & $0 \times 02$ & File ID ( 2 bytes) & - \\
\hline $0 \times 0 D$ & $\begin{array}{l}\text { SELECT EF FILE } \\
\text { UNDER } \\
\text { SELECTED DF }\end{array}$ & $0 \times 00$ & $0 \times A 4$ & $0 \times 02$ & $\begin{array}{l}0 \times 00 \\
\text { or } \\
0 \times 0 C\end{array}$ & $0 \times 02$ & File ID ( 2 bytes) & - \\
\hline $0 \times 0 C$ & SELECT FILE & $0 \times 00$ & $0 \times A 4$ & $\begin{array}{l}0 \times 00- \\
0 \times 03\end{array}$ & $\begin{array}{l}0 \times 00 \\
\text { or } \\
0 \times 0 C\end{array}$ & $0 \times 02$ & File ID ( 2 bytes) & - \\
\hline $0 \times 0 E$ & $\begin{array}{l}\text { SELECT MASTER } \\
\text { FILE (Root) }\end{array}$ & $0 \times 00$ & $0 \times A 4$ & $0 \times 03$ & $\begin{array}{l}0 \times 00 \\
\text { or } \\
0 \times 0 C\end{array}$ & $0 \times 02$ & File ID ( 2 bytes) & - \\
\hline $0 \times 03$ & UPDATE BINARY & $0 \times 00$ & $0 \times \mathrm{D} 6$ & $\mathrm{Off} / \mathrm{H}$ & Off/L & $L_{c}$ & Data to Update & - \\
\hline
\end{tabular}




\subsubsection{Get Response APDU}

This APDU is used to read smart card results available from the completion of the previously executed APDU.

\section{Command Message}

\begin{tabular}{|l|l|}
\hline Function Code & $0 \times 07$ \\
\hline CLA & $0 \times 00$ \\
\hline INS & $0 \times C 0$ \\
\hline P1 & $0 \times 00$ \\
\hline P2 & $0 \times 00$ \\
\hline $\mathbf{L}_{c}$ & Empty \\
\hline Data Field & Empty \\
\hline $\mathbf{L}_{\mathbf{e}}$ & Number of bytes to read in response \\
\hline
\end{tabular}

\section{Response Message}

\section{Data Field returned in the Response Message}

If the immediately preceding APDU has indicated that additional data is available, the data field of an immediately following Get Response APDU will contain this data.

\section{Processing State returned in the Response Message}

\begin{tabular}{|c|c|l|}
\hline SW1 & SW2 & \multicolumn{1}{c|}{ Meaning } \\
\hline 61 & $\mathrm{XX}$ & $\begin{array}{l}\text { Normal processing, } \mathrm{XX} \text { still available to read with subsequent Get } \\
\text { Response }\end{array}$ \\
\hline 62 & 81 & Part of returned data may be corrupted \\
\hline 67 & 00 & Wrong length (incorrect $\mathrm{L}_{\mathrm{e}}$ field) \\
\hline $6 \mathrm{~A}$ & 86 & Incorrect parameters $\mathrm{P1}-\mathrm{P} 2$ \\
\hline $6 \mathrm{C}$ & $\mathrm{XX}$ & Wrong length (wrong $\mathrm{L}_{\mathrm{e}}$ field; $\mathrm{XX}$ indicates the exact length) \\
\hline 90 & 00 & Successful execution \\
\hline
\end{tabular}




\subsubsection{Read Binary APDU}

This APDU is used to read the currently selected transparent file. All access control operations necessary for reading the file must be completed before using this APDU.

\section{Command Message}

\begin{tabular}{|l|l|}
\hline Function Code & 0x02 \\
\hline CLA & 0x00 \\
\hline INS & 0xB0 \\
\hline P1 & High-order byte of 2-byte offset \\
\hline P2 & Low-order byte of 2-byte offset \\
\hline $\mathbf{L}_{c}$ & Empty \\
\hline Data Field & Empty \\
\hline $\mathbf{L}_{\mathbf{e}}$ & Number of bytes to read \\
\hline
\end{tabular}

\section{Response Message}

\section{Data Field returned in the Response Message}

$\mathrm{L}_{\mathrm{e}}$ number of bytes followed by the two-byte processing state.

\section{Processing State returned in the Response Message}

\begin{tabular}{|c|c|l|}
\hline SW1 & SW2 & \multicolumn{1}{|c|}{ Meaning } \\
\hline 62 & 81 & Part of returned data may be corrupted \\
\hline 62 & 82 & End of file reached before reading $L_{e}$ bytes \\
\hline 67 & 00 & Wrong length (wrong $L_{e}$ field) \\
\hline 69 & 81 & Command incompatible with file structure \\
\hline 69 & 82 & Security status not satisfied \\
\hline 69 & 86 & Command not allowed (no current EF) \\
\hline $6 \mathrm{~A}$ & 81 & Function not supported \\
\hline $6 \mathrm{~A}$ & 82 & File not found \\
\hline $6 \mathrm{~B}$ & 00 & Wrong parameters (offset outside the $E F$ ) \\
\hline $6 \mathrm{C}$ & $\mathrm{XX}$ & Wrong length (wrong $\mathrm{L}_{\mathrm{e}}$ field; $X X$ indicates the exact length) \\
\hline 90 & 00 & Successful execution \\
\hline
\end{tabular}




\subsubsection{SELECT DF APDU}

Sets the currently selected dedicated file to a dedicated file contained in the currently selected dedicated file.

Command Message

\begin{tabular}{|l|l|}
\hline Function Code & 0x01 \\
\hline CLA & 0x00 \\
\hline INS & 0xA4 \\
\hline P1 & 0x01 - Select child DF of current DF \\
\hline P2 & 0x00 for response required, 0x0C for no response required \\
\hline$L_{c}$ & $0 \times 02$ \\
\hline Data Field & 2-byte File Identifier \\
\hline$L_{e}$ & Number of bytes returned \\
\hline
\end{tabular}

\section{Response Message}

\section{Data Field returned in the Response Message}

If $\mathrm{P} 2$ is set to 0x00, data is returned as per ISO 7816-4 [ISO4]. If $\mathrm{P} 2$ is set to 0x0C, no data is returned.

\section{Processing State returned in the Response Message}

\begin{tabular}{|c|c|l|}
\hline SW1 & SW2 & \multicolumn{1}{|c|}{ Meaning } \\
\hline 62 & 83 & Selected file deactivated \\
\hline 62 & 84 & File control information not formatted according to ISO 7816-4. \\
\hline $6 \mathrm{~A}$ & 81 & Function not supported \\
\hline $6 \mathrm{~A}$ & 82 & File not found \\
\hline $6 \mathrm{~A}$ & 86 & Incorrect parameters P1-P2 \\
\hline $6 \mathrm{~A}$ & 87 & L $_{\mathrm{c}}$ inconsistent with P1-P2 \\
\hline 90 & 00 & Successful execution \\
\hline
\end{tabular}




\subsection{Select EF Under Selected DF APDU}

This APDU selects an Elementary File under the currently selected DF.

\section{Command Message}

\begin{tabular}{|l|l|}
\hline Function Code & 0x0D \\
\hline CLA & 0x00 \\
\hline INS & 0xA4 \\
\hline P1 & 0x02 - Select child EF of current DF \\
\hline P2 & 0x00 for response required, 0x0C for no response required \\
\hline$L_{c}$ & 0x02 \\
\hline Data Field & 2-byte File Identifier \\
\hline$L_{e}$ & Number of bytes returned \\
\hline
\end{tabular}

\section{Response Message}

\section{Data Field returned in the Response Message}

If $\mathrm{P} 2$ is set to 0x00, data is returned as per ISO 7816-4 [ISO4]. If $\mathrm{P} 2$ is set to $0 \mathrm{x} 0 \mathrm{C}$, no data is returned.

Processing State returned in the Response Message

\begin{tabular}{|c|c|l|}
\hline SW1 & SW2 & \multicolumn{1}{|c|}{ Meaning } \\
\hline 62 & 83 & Selected file deactivated \\
\hline 62 & 84 & $\begin{array}{l}\text { File control information not formatted according to ISO 7816-4, } \\
\text { Section 5.1.5 }\end{array}$ \\
\hline $6 \mathrm{~A}$ & 81 & Function not supported \\
\hline $6 \mathrm{~A}$ & 82 & File not found \\
\hline $6 \mathrm{~A}$ & 86 & Incorrect parameters P1-P2 \\
\hline $6 \mathrm{~A}$ & 87 & $\mathrm{~L}_{\mathrm{c}}$ inconsistent with P1-P2 \\
\hline 90 & 00 & Successful execution \\
\hline
\end{tabular}




\subsubsection{Select File APDU}

This APDU works as described in ISO 7816-4 [ISO4] to select the master file, a DF, or an EF.

\section{Command Message}

\begin{tabular}{|l|l|}
\hline Function Code & 0x0C \\
\hline CLA & 0x00 \\
\hline INS & 0xA4 \\
\hline P1 & See below \\
\hline P2 & 0x00 for response required, 0x0C for no response required \\
\hline$L_{c}$ & Number of bytes in File Identifier, i.e., 2 \\
\hline Data Field & File Identifier \\
\hline$L_{e}$ & Empty \\
\hline
\end{tabular}

P1: $\quad$ 0x00 Explicit selection with Data Field; Data field must contain a valid File Identifier

0x01 Select child DF of current DF; Data Field must contain a valid File Identifier

0x02 Select child EF of current DF; Data Field must contain a valid File Identifier

0x03 Select parent DF of current DF; empty Data Field

\section{Response Message}

\section{Data Field returned in the Response Message}

If $\mathrm{P} 2$ is set to 0x00, data is returned as per ISO 7816-4 [ISO4]. If P2 is set to 0x0C, no data is returned.

\section{Processing State returned in the Response Message}

\begin{tabular}{|c|c|l|}
\hline SW1 & SW2 & \multicolumn{1}{|c|}{ Meaning } \\
\hline 62 & 83 & Selected file deactivated \\
\hline 62 & 84 & FCl not formatted according to ISO 7816-4 Section 5.1.5 \\
\hline $6 \mathrm{~A}$ & 81 & Function not supported \\
\hline $6 \mathrm{~A}$ & 82 & File not found \\
\hline $6 \mathrm{~A}$ & 86 & Incorrect parameters P1-P2 \\
\hline $6 \mathrm{~A}$ & 87 & $\mathrm{~L}_{\mathrm{c}}$ inconsistent with P1-P2 \\
\hline 90 & 00 & Successful execution \\
\hline
\end{tabular}




\subsubsection{Select Master File APDU}

This APDU selects the Master File or the root of a file system card directory structure.

\section{Command Message}

\begin{tabular}{|l|l|}
\hline Function Code & 0x0E \\
\hline CLA & 0x00 \\
\hline INS & 0xA4 \\
\hline P1 & 0x03 - Select MF \\
\hline P2 & 0x00 for response required, 0x0C for no response required \\
\hline$L_{c}$ & 0x02 \\
\hline Data Field & File Identifier \\
\hline$L_{e}$ & Empty \\
\hline
\end{tabular}

\section{Response Message}

\section{Data Field returned in the Response Message}

If $\mathrm{P} 2$ is set to 0x00, data is returned as per ISO 7816-4 [ISO4]. If $\mathrm{P} 2$ is set to 0x0C, no data is returned.

\section{Processing State returned in the Response Message}

\begin{tabular}{|c|c|l|}
\hline SW1 & SW2 & \multicolumn{1}{|c|}{ Meaning } \\
\hline 62 & 83 & Selected file deactivated \\
\hline 62 & 84 & FCl not formatted according to ISO 7816-4 Section 5.1.5 \\
\hline $6 \mathrm{~A}$ & 81 & Function not supported \\
\hline $6 \mathrm{~A}$ & 82 & File not found \\
\hline $6 \mathrm{~A}$ & 86 & Incorrect parameters P1-P2 \\
\hline $6 \mathrm{~A}$ & 87 & $\mathrm{~L}_{\mathrm{c}}$ inconsistent with P1-P2 \\
\hline 90 & 00 & Successful execution \\
\hline
\end{tabular}




\subsubsection{Update Binary APDU}

This APDU is used to update the currently selected transparent file. All access control operations necessary for writing to the selected file must be completed before using this APDU.

\section{Command Message}

\begin{tabular}{|l|l|}
\hline Function Code & $0 \times 03$ \\
\hline CLA & $0 \times 00$ \\
\hline INS & 0xD6 \\
\hline P1 & High-order byte of 2-byte offset \\
\hline P2 & Low-order byte of 2-byte offset \\
\hline $\mathbf{L}_{c}$ & Number of bytes to update \\
\hline Data Field & New data to be used to replace existing data \\
\hline $\mathbf{L}_{e}$ & Empty \\
\hline
\end{tabular}

\section{Response Message}

\section{Data Field returned in the Response Message}

Empty.

Processing State returned in the Response Message

\begin{tabular}{|c|c|l|}
\hline SW1 & SW2 & \multicolumn{1}{|c|}{ Meaning } \\
\hline 63 & CX & Successful updating after $X$ retries, X=0 means no counter provided \\
\hline 65 & 81 & Memory failure (unsuccessful updating) \\
\hline 67 & 00 & Wrong length (wrong Lc field) \\
\hline 69 & 81 & Command incompatible with file structure \\
\hline 69 & 82 & Security status not satisfied \\
\hline 69 & 86 & Command not allowed (no current EF) \\
\hline $6 \mathrm{~A}$ & 81 & Function not supported \\
\hline $6 \mathrm{~A}$ & 82 & File not found \\
\hline $6 \mathrm{~B}$ & 00 & Wrong parameters (offset outside the EF) \\
\hline 90 & 00 & Successful execution \\
\hline
\end{tabular}




\subsubsection{Access Control APDUs}

Table 5-5 shows the Access Control APDU set for file system and VM cards. The Access Control APDUs assume that the default cryptographic algorithm is DES3-ECB, with a double length key-size, 16 bytes.

Table 5-5: Access Control APDUs

\begin{tabular}{|c|l|c|c|c|c|c|c|c|}
\hline \multicolumn{10}{|c|}{ Access Control APDUs } \\
\hline FC & Card Function & CLA & INS & P1 & P2 & $L_{c}$ & Data & $L_{e}$ \\
\hline 0x0A & $\begin{array}{l}\text { EXTERNAL } \\
\text { AUTHENTICATE }\end{array}$ & $0 \times 00$ & $0 \times 82$ & AlgID & Key \# & $L_{c}$ & Cryptogram & - \\
\hline 0x05 & GET CHALLENGE & $0 \times 00$ & $0 \times 84$ & $0 \times 00$ & $0 \times 00$ & - & - & $L_{e}$ \\
\hline 0x09 & $\begin{array}{l}\text { INTERNAL } \\
\text { AUTHENTICATE }\end{array}$ & $0 \times 00$ & $0 \times 88$ & AlgID & Key \# & $\mathrm{L}_{c}$ & Challenge & $\mathrm{L}_{e}$ \\
\hline 0x08 & VERIFY & $0 \times 00$ & $0 \times 20$ & $0 \times 00$ & $\mathrm{CHV}$ & $\mathrm{L}_{c}$ & $\begin{array}{c}\text { Authentication } \\
\text { data }\end{array}$ & - \\
\hline
\end{tabular}

Various smart cards perform external and internal authentication in similar but slightly different ways. The general methods used by the default GSC-IS APDU set are described below. To change the syntax and behavior of the default APDUs, the appropriate descriptor codes can be used in conjunction with command and response code tuples in the $\mathrm{CCC}$ as described in $\underline{\text { Chapter } 6 .}$

\section{External Authentication Method:}

1. The client application and the smart card share a secret key; the smart card may store the key in a key file.

2. The SPS instructs the smart card to issue an 8-byte challenge via the GET CHALLENGE APDU; the smart card returns the challenge to the SPS.

3. The client application encrypts the challenge with its secret key to produce a cryptogram.

4. The SPS sends the cryptogram to the smart card and possibly the key number via the EXTERNAL AUTHENTICATE APDU.

5. The smart card accesses the specified secret key, its saved copy of the challenge, and computes the same cryptogram and returns a status code to the SPS.

6. If the status code indicates that the cryptograms match, external authentication is successful.

\section{Internal Authentication Method:}

Step 1: PIN authentication

1. The client application and the smart card share a PIN; the smart card may store the PIN in a PIN file.

2. The SPS sends the PIN and the PIN number to the smart card via the VERIFY APDU. 
3. The smart card accesses the specified PIN, compares it to the client application's PIN, and returns a status code to the SPS.

4. If the status code indicates that the PINs match, the smart card will permit the internal authentication to proceed.

Step 2: Internal Authentication

1. The client application and the smart card share a secret key; the smart card may store the key in a key file.

2. The client application computes an 8-byte challenge and sends this to the smart card along with the key number via the INTERNAL AUTHENTICATION APDU.

3. The smart card accesses the specified secret key, the challenge, and computes the same cryptogram.

4. The SPS retrieves the cryptogram in the response to the INTERNAL AUTHENTICATION APDU.

5. If the cryptograms match, internal authentication is successful.

\section{Algorithm Identifiers for EXTERNAL and INTERNAL AUTHENTICATE APDUs:}

ISO 7816-4 [ISO4] does not define algorithm identifiers for EXTERNAL and INTERNAL AUTHENTICATE, therefore this specification defines them in Table 5-6. If a smart card does not use the algorithm identifiers defined in Table 5-6, then the appropriate definitions of the EXTERNAL and INTERNAL AUTHENTICATE APDUs in the CCC command tuples will be required. If the smart card supports multiple cryptographic algorithms for this command, then successive tuples can be used to identify all the possible cryptographic algorithms and their corresponding P1 values.

Table 5-6: Algorithm Identifiers for Authentication APDUs

\begin{tabular}{|c|c|c|}
\hline Algorithm Identifier & Algorithm-Mode & Key Length in Bits \\
\hline 0x00 & Triple DES-ECB & 128 \\
\hline 0x01 & Triple DES-CBC & 128 \\
\hline 0x02 & DES-ECB & 64 \\
\hline 0x03 & DES-CBC & 64 \\
\hline $0 \times 04$ & RSA & 512 \\
\hline 0x05 & RSA & 768 \\
\hline 0x06 & RSA & 1024 \\
\hline 0x07 & (Reserved for RSA 2048) & $(2048)$ \\
\hline 0x08 & AES-ECB & 128 \\
\hline 0x09 & AES-CBC & 128 \\
\hline 0x0A & AES-ECB & 192 \\
\hline 0x0B & AES-CBC & 192 \\
\hline 0x0C & AES-ECB & 256 \\
\hline 0x0D & AES-CBC & 256 \\
\hline
\end{tabular}


NIST IR 6887 - 2003 EDITION, GSC-IS VERSION 2.1

\begin{tabular}{|c|c|c|}
\hline Algorithm Identifier & Algorithm-Mode & Key Length in Bits \\
\hline $0 \times 0 \mathrm{E}$ & RFU & - \\
\hline $0 \times 0 \mathrm{~F}$ & RFU & - \\
\hline
\end{tabular}

NOTE: High nibble of the Algorithm Identifier shall be zero. 


\subsubsection{External Authenticate APDU}

This APDU is used in conjunction with the GET CHALLENGE APDU to authenticate a client application to the smart card. GET CHALLENGE would be issued first to cause the smart card to issue a random number, i.e., the challenge. The client application would encrypt the challenge and send the resultant cryptogram to the smart card via the EXTERNAL AUTHENTICATE APDU. The smart card would then decrypt it using the same algorithm as the client application and compare it to its internally stored copy of the challenge. If the cryptograms match, the client application is authenticated to the smart card. If the cryptograms do not match, the challenge is no longer valid.

\section{Command Message}

\begin{tabular}{|l|l|}
\hline Function Code & 0x0A \\
\hline CLA & 0x00 \\
\hline INS & 0x82 \\
\hline P1 & Algorithm Identifier - see Table 5-6 \\
\hline P2 & 0x00 for default key, 0x01 to 0x30 for key number \\
\hline $\mathbf{L}_{c}$ & Length of data field \\
\hline Data Field & Cryptogram \\
\hline $\mathbf{L}_{e}$ & Empty \\
\hline
\end{tabular}

\section{Response Message}

\section{Data Field returned in the Response Message}

Empty.

Processing State returned in the Response Message

\begin{tabular}{|c|c|l|}
\hline SW1 & SW2 & \multicolumn{1}{c|}{ Meaning } \\
\hline 63 & 00 & No information given (Authentication failed) \\
\hline 63 & $\mathrm{CX}$ & Authentication failed; X indicated number of further allowed retries \\
\hline 67 & 00 & Wrong length (the Lc field is incorrect) \\
\hline 69 & 83 & Authentication method blocked \\
\hline 69 & 84 & Referenced data deactivated \\
\hline 69 & 85 & $\begin{array}{l}\text { Conditions of use not satisfied (the command is not allowed in this } \\
\text { context) }\end{array}$ \\
\hline $6 \mathrm{~A}$ & 86 & Incorrect parameters P1-P2 \\
\hline $6 \mathrm{~A}$ & 88 & Referenced data not found \\
\hline 90 & 00 & Successful execution \\
\hline
\end{tabular}




\subsubsection{Get Challenge APDU}

This APDU is used to cause the smart card to generate a cryptographic challenge, e.g., a random number, for use in the subsequent security related procedure such as EXTERNAL AUTHENTICATE. The smart card saves a copy of the challenge internally until the completion of the security related procedure or an error occurs.

The challenge is valid only for the next APDU in the same card session.

\section{Command Message}

\begin{tabular}{|l|l|}
\hline Function Code & 0x05 \\
\hline CLA & $0 \times 00$ \\
\hline INS & $0 \times 84$ \\
\hline P1 & $0 \times 00$ \\
\hline P2 & $0 \times 00$ \\
\hline $\mathbf{L}_{c}$ & Empty \\
\hline Data Field & Empty \\
\hline $\mathbf{L}_{\mathbf{e}}$ & Length in bytes of expected random challenge \\
\hline
\end{tabular}

\section{Response Message}

\section{Data Field returned in the Response Message}

If the APDU result indicates success, $\mathrm{L}_{\mathrm{e}}$ number of bytes will be available to read from the smart card, i.e., the 8-byte challenge.

Processing State returned in the Response Message

\begin{tabular}{|c|c|l|}
\hline SW1 & SW2 & \multicolumn{1}{|c|}{ Meaning } \\
\hline $6 \mathrm{~A}$ & 81 & Function not supported \\
\hline $6 \mathrm{~A}$ & 86 & Incorrect parameters P1-P2 \\
\hline 90 & 00 & Successful execution \\
\hline
\end{tabular}




\subsubsection{Internal Authenticate APDU}

This APDU is used to authenticate the smart card to the client application. An 8-byte challenge is computed by the client application and then passed to the smart card via this command. Also passed are a key number and the cryptographic algorithm the smart card uses when encrypting the challenge. The smart card takes this information and encrypts the challenge according to the algorithm specified and the specified key and returns the resultant cryptogram. If the decrypted cryptogram from the smart card matches the initial challenge computed by the client application, the smart card is authenticated to the client application.

\section{Command Message}

\begin{tabular}{|l|l|}
\hline Function Code & 0x09 \\
\hline CLA & 0x00 \\
\hline INS & 0x88 \\
\hline P1 & Algorithm Identifier - see Table 5-6 \\
\hline P2 & 0x00 for default key, 0x01 to 0x30 for key number \\
\hline $\mathbf{L}_{c}$ & Length of data field \\
\hline Data Field & Challenge \\
\hline $\mathrm{L}_{e}$ & Length of expected cryptogram \\
\hline
\end{tabular}

\section{Response Message}

\section{Data Field returned in the Response Message}

The cryptogram.

\section{Processing State returned in the Response Message}

\begin{tabular}{|c|c|l|}
\hline SW1 & sW2 & \multicolumn{1}{|c|}{ Meaning } \\
\hline 69 & 84 & Referenced data deactivated \\
\hline 69 & 85 & Conditions of use not satisfied \\
\hline $6 \mathrm{~A}$ & 86 & Incorrect parameters P1-P2 \\
\hline $6 \mathrm{~A}$ & 88 & Reference data not found \\
\hline 90 & 00 & Successful execution \\
\hline
\end{tabular}




\subsubsection{Verify APDU}

This APDU is used to compare authentication data such as a password, key or PIN with corresponding authentication data on the smart card. The SPS sends the authentication data in this APDU and directs the smart card to compare it with authentication data on the smart card. The authentication data is passed unencrypted.

\section{Command Message}

\begin{tabular}{|l|l|}
\hline Function Code & 0x08 \\
\hline CLA & 0x00 \\
\hline INS & $0 \times 20$ \\
\hline P1 & $0 \times 00$ \\
\hline P2 & 0x00 for default key, 0x01 to 0x30 for key number \\
\hline$L_{c}$ & Length of data field \\
\hline Data Field & Authentication data (i.e., password or PIN) \\
\hline$L_{e}$ & Empty \\
\hline
\end{tabular}

Note: If the Lc is 0x00 and the Data Field is empty, VERIFY returns the number of tries remaining on the referenced PIN.

\section{Response Message}

Data Field returned in the Response Message

Empty.

\section{Processing State returned in the Response Message}

\begin{tabular}{|c|c|l|}
\hline SW1 & SW2 & \multicolumn{1}{|c|}{ Meaning } \\
\hline 63 & 00 & Verification failed \\
\hline 63 & $\mathrm{CX}$ & Verification failed, X indicates the number of further allowed retries \\
\hline 69 & 83 & Authentication method blocked \\
\hline 69 & 84 & Referenced data deactivated \\
\hline $6 \mathrm{~A}$ & 86 & Incorrect parameters P1-P2 \\
\hline $6 \mathrm{~A}$ & 88 & Reference data not found \\
\hline 90 & 00 & Successful execution \\
\hline
\end{tabular}




\subsubsection{Public Key Operations APDUs}

Table 5-7 shows the public key operations APDUs for file system and VM cards. The default padding scheme for RSA is assumed to be RSA_NO_PAD. The computation is performed with the private key.

Table 5-7: Public Key Operations APDUs

\begin{tabular}{|c|l|c|c|c|c|c|c|c|}
\hline \multicolumn{1}{|c|}{ Public Key Operations APDU } \\
\hline FC & Card Function & CLA & INS & P1 & P2 & $L_{c}$ & Data & $L_{e}$ \\
\hline 0x05 & $\begin{array}{l}\text { MANAGE } \\
\text { SECURITY } \\
\text { ENVIRONMENT }\end{array}$ & $0 \times 00$ & $0 \times 22$ & $0 \times 41$ & $0 \times B 6$ & $L_{c}$ & $\begin{array}{l}\text { Key Reference } \\
\text { information }\end{array}$ & - \\
\hline 0x0B & $\begin{array}{l}\text { PERFORM } \\
\text { SECURITY } \\
\text { OPERATION }\end{array}$ & 0x00 & 0x2A & 0x9E & 0x9A & $L_{c}$ & $\begin{array}{l}\text { Message digest } \\
\text { to sign }\end{array}$ & $L_{e}$ \\
\hline
\end{tabular}




\subsubsection{Manage Security Environment APDU}

This APDU is used to initiate the computation of a digital signature on a message by setting a digital signature template to be used by a subsequent PERFORM SECURITY OPERATION APDU.

\section{Command Message}

\begin{tabular}{|l|l|}
\hline Function Code & 0x05 \\
\hline CLA & $0 \times 00$ \\
\hline INS & $0 \times 22$ \\
\hline P1 & $0 \times 41$ \\
\hline P2 & 0xB6 \\
\hline$L_{c}$ & $L_{c}=$ Message length in bytes \\
\hline Data Field & Key Reference information \\
\hline$L_{e}$ & Empty \\
\hline
\end{tabular}

Data Field: $\quad$ Key reference information, formatted as per ISO 7816-8 [ISO8].

\section{Response Message}

Data Field returned in the Response Message

Empty.

Processing State returned in the Response Message

\begin{tabular}{|c|c|l|}
\hline SW1 & SW2 & \multicolumn{1}{c|}{ Meaning } \\
\hline 66 & 00 & The Security Environment cannot be set \\
\hline 67 & 00 & Wrong length (the Lc field incorrect) \\
\hline $6 \mathrm{~A}$ & 80 & $\begin{array}{l}\text { Invalid or missing tag, length or value in a Control Reference Data } \\
\text { Object (CRDO) }\end{array}$ \\
\hline $6 \mathrm{~A}$ & 86 & Incorrect parameters P1-P2 \\
\hline 90 & 00 & Successful execution \\
\hline
\end{tabular}




\subsubsection{Perform Security Operation APDU}

This APDU is used to initiate the computation of a digital signature on a message digest. This APDU responds with the computed signature.

\section{Command Message}

\begin{tabular}{|l|l|}
\hline Function Code & 0x0B \\
\hline CLA & $0 \times 00$ \\
\hline INS & 0x2A \\
\hline P1 & 0x9E \\
\hline P2 & 0x9A \\
\hline$L_{c}$ & Length in bytes of message digest \\
\hline Data Field & Message digest to sign \\
\hline$L_{e}$ & Length of response \\
\hline
\end{tabular}

\section{Response Message}

\section{Data Field returned in the Response Message}

The signed message digest.

\section{Processing State returned in the Response Message}

\begin{tabular}{|c|c|l|}
\hline SW1 & SW2 & \multicolumn{1}{c|}{ Meaning } \\
\hline 67 & 00 & Wrong length (the Lc field is incorrect) \\
\hline 69 & 81 & Invalid file type \\
\hline 69 & 85 & No preceding MSE-Set or previously specified key file is missing \\
\hline 69 & 87 & Missing Secure Messaging Data Object \\
\hline 69 & 88 & Incorrect Secure Message Data Object \\
\hline $6 \mathrm{~A}$ & 86 & Incorrect parameters P1-P2 \\
\hline 90 & 00 & Successful execution \\
\hline $6 \mathrm{C}$ & $\mathrm{XX}$ & Wrong length (wrong $\mathrm{L}_{\mathrm{e}}$ field; $\mathrm{XX}$ indicates the exact length) \\
\hline
\end{tabular}




\subsection{Mapping Default APDUs to Native APDU Sets}

For file system cards that contain a native APDU instruction set that differs from the GSC-IS default set, the SPS must implement a mapping mechanism to translate the default APDUs into the native APDUs in accordance with the information obtained from the CCC.

\subsubsection{The CCC Command and Response Tuples}

The CCC is a file that must be present on each conformant GSC-IS smart card. The CCC includes a set of tuples, which are 2-byte values that describe the differences in syntax between a file system card's native APDU set and the GSC-IS APDU set. Chapter 6 describes the contents of the CCC in more detail. Besides syntactical differences, the tuples also describe differences in APDU execution and data format. The codes used in the tuples to describe these differences are called Descriptor Codes.

As an example, Descriptor Codes can be used to indicate that a smart card's native READ BINARY APDU requires that offsets be on word boundaries as opposed to byte boundaries. Or, a smart card's native EXTERNAL AUTHENTICATE APDU may require 4 bytes of a cryptographic challenge whereas the default APDU requires 8 bytes. A descriptor code can be used to indicate that the SPS must build and send an APDU using a 4-byte cryptographic challenge.

A smart card with a native APDU instruction set identical to the GSC-IS APDU set would still contain a CCC, however the CCC would contain no tuples (and descriptor codes), since no APDU mapping would be necessary.

\subsubsection{Native APDU Mapping and CCC Grammar}

Each conformant SPS for file system cards must implement the translation or mapping mechanism to translate the default GSC-IS APDU set into a smart card's native APDU set both in syntax and in operation. The SPS performs this translation according to the rules of a CCC grammar associated with the set of tuples located in the smart card's CCC, described in more detail in Chapter 6.

The card edge interface for file system cards operates as follows:

1. A smart card vendor creates a CCC and loads it onto a smart card.

2. The SPS has knowledge of the default GSC-IS APDUs and how to translate them into a conformant card's native APDU set using the CCC grammar.

3. The smart card, when ready for use, is inserted into a reader.

4. The SPS's card edge locates and reads the contents of the CCC.

5. The SPS's card edge maps the default APDU set into the card's native set using the tuples in the $\mathrm{CCC}$ and the associated $\mathrm{CCC}$ grammar.

6. The SPS, when sending APDUs to the smart card, then uses the smart card's native ADPU set according to its rules of operation. 


\subsubsection{Detecting Card APDUs}

The SPS can detect which of the default GSC-IS APDUs are available on a smart card according to the following rules:

1. If the APDU is defined in a capability tuple as not implemented (via Descriptor Code 0xFE, see Table 6-10), then the APDU is not available.

2. If the APDU is defined otherwise in one or more capability tuples, the APDU is available as defined.

3. If the APDU is not defined in any capability tuple, the APDU is assumed to be available and operates as described in this specification and in ISO 7816-4 [ISO4] and 7816-8 [ISO8].

The CCC optionally may contain a six-byte CARD APDUs bit-string for the purposes of informing the SPS which ISO 7816-4 [ISO4] and 7816-8 [ISO8] APDUs are available on the smart card. Each bit in the string, if set to 1, would indicate the presence of a corresponding APDU; a ' 0 ' would indicate the corresponding APDU is not present or is not to be used. The CARD APDUs string does not override any command tuples; however, if an APDU is described in command tuples but not in the CARD APDUs field, the command tuples are to be used. Table 5-8 shows bit positions and corresponding APDUs.

Table 5-8: CARD APDUs Values

\begin{tabular}{|c|c|}
\hline $\begin{array}{l}\text { Bit } \\
\text { Position }\end{array}$ & 7816-4 APDU \\
\hline 0 & Reserved, Used for Shift Operation (see Section 6.4.2) \\
\hline 1 & Select DF \\
\hline 2 & Transparent Read (Binary) \\
\hline 3 & Update Binary File \\
\hline 4 & RFU \\
\hline 5 & Manage Security Environment \\
\hline 6 & Get Challenge \\
\hline 7 & Get Response \\
\hline 8 & Verify (CHV) \\
\hline 9 & Internal Authenticate \\
\hline 10 & External Authenticate \\
\hline 11 & Perform Security Operation \\
\hline 12 & Select File \\
\hline 13 & Select EF (under current DF) \\
\hline 14 & Select MF (root) \\
\hline 15 & RFU \\
\hline
\end{tabular}




\subsubsection{Default Status Code Responses}

The default APDUs return status codes according to ISO 7816-4 [ISO4]. Non-ISO card-specific status codes can be mapped into a GSC-IS set of status code responses, shown in Table 5-9. As described in

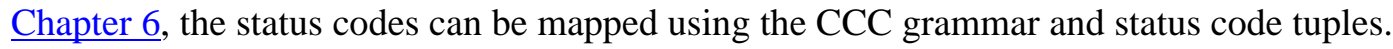

Table 5-9: GSC-IS Status Code Responses

\begin{tabular}{|l|l|}
\hline \multicolumn{2}{|c|}{ Status Conditions } \\
\hline $0 \times 00$ & Successful Completion \\
\hline $0 \times 01$ & Successful Completion - Warning 1 \\
\hline 0x02 & Successful Completion - Warning 2 \\
\hline 0x03 & Reserved \\
\hline 0x04 & Reserved \\
\hline 0x05 & Reserved \\
\hline 0x06 & Reserved \\
\hline 0x07 & Reserved \\
\hline 0x08 & Access Condition not Satisfied \\
\hline 0x09 & Function not Allowed \\
\hline 0x0A & Inconsistent Parameter \\
\hline 0x0B & Data Error \\
\hline 0x0C & Wrong Length \\
\hline 0x0D & Function not compatible with file structure \\
\hline 0x0E & File/Record not Found \\
\hline 0x0F & Function Not Supported \\
\hline
\end{tabular}

\subsection{Card Edge Interface for VM Cards}

The Card Edge Interface for VM Cards is made up of provider modules that provide three classes of services: generic container management services, symmetric key cryptographic services, and public (asymmetric) key cryptographic services. Each provider module may provide one or more class of service. These provider modules are implemented as on-card applets. For virtual machine cards, the terms "provider" and "applet" are synonymous.

Common interface methods that must be implemented by all providers are described first. The six APDUs listed in Table 5-12 must be implemented by all providers. The methods unique to each provider class are described in subsequent sections. Table 5-10 provides a summary of the APDUs implemented for the virtual machine card edge.

Table 5-10: Virtual Machine Card Edge APDUs

\begin{tabular}{|l|l|}
\hline \multicolumn{2}{|c|}{ Virtual Machine APDU Set } \\
\hline $\begin{array}{l}\text { Common Interface Methods } \\
\text { VM APDUs }\end{array}$ & SELECT APPLET \\
\cline { 2 - 2 } & SELECT OBJECT
\end{tabular}




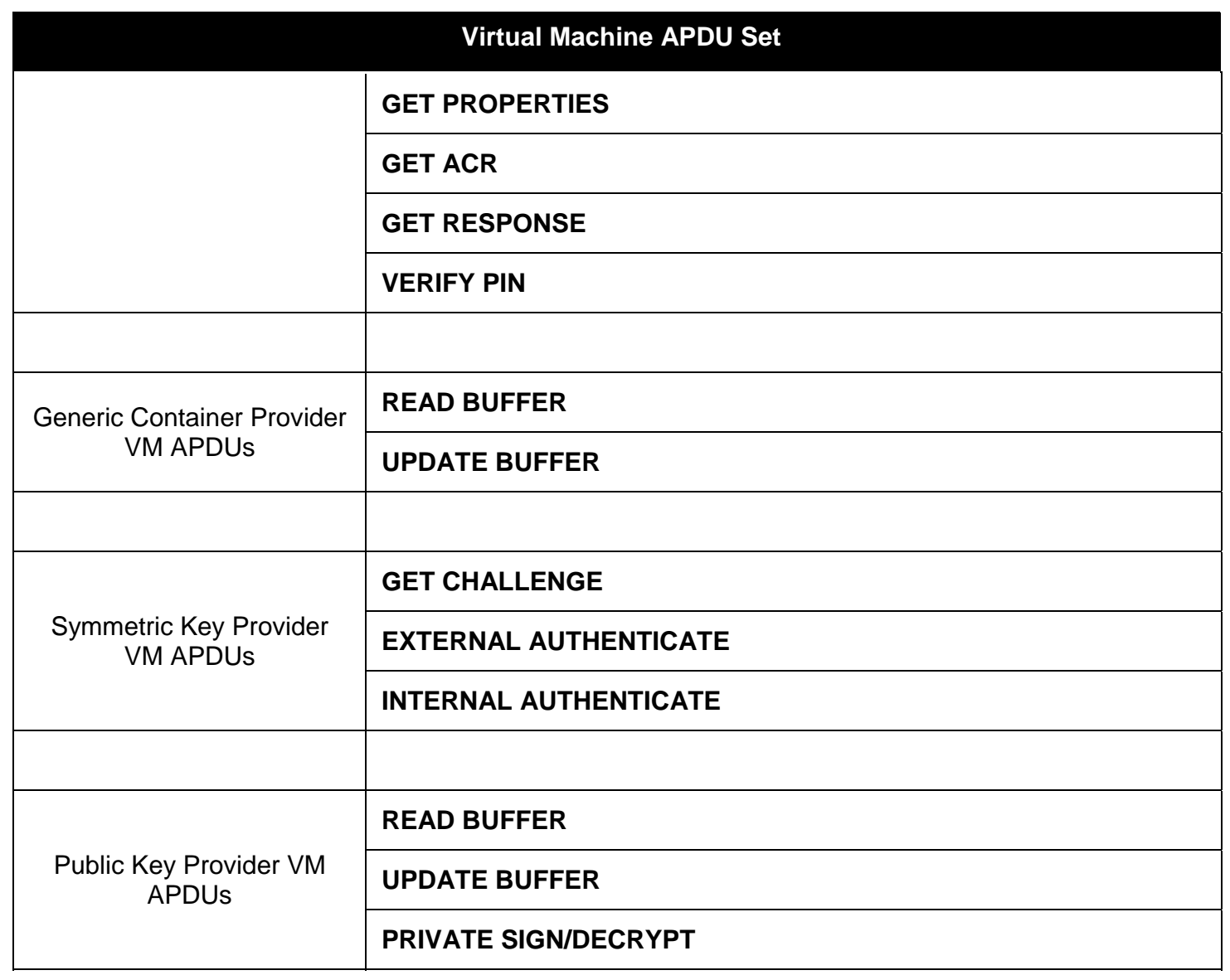

\subsubsection{Virtual Machine Card Access Control Rule Configuration}

Each smart card service provider shall present its services through a set of APDUs implemented and managed by the provider. The ACRs associated with card level services vary depending on the application.

ACRs shall be coded as a single byte value (range 0x00 - 0xFF) as defined in Table 3-2.

\subsubsection{Virtual Machine Card Edge General Error Conditions}

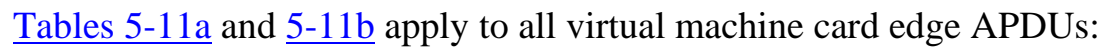

Table 5-11a: Successful Conditions

\begin{tabular}{|c|c|}
\hline $\begin{array}{c}\text { Status bytes } \\
\text { SW1 SW2 }\end{array}$ & Meaning \\
\hline $61 \mathrm{LL}$ & SW2 indicates the number of response bytes available \\
\hline 9000 & Normal ending of the command \\
\hline
\end{tabular}


Table 5-11b: General Error Conditions

\begin{tabular}{|c|l|}
\hline $\begin{array}{c}\text { Status bytes } \\
\text { SW1 SW2 }\end{array}$ & \\
\hline 62 00 & Applet or instance logically deleted \\
\hline 63 CX & Authentication failed, X indicates the remaining tries \\
\hline 6581 & Memory failure \\
\hline 6700 & Incorrect parameter Lc \\
\hline 6 C XX & Wrong length in Le parameter, SW2 indicates the exact length \\
\hline 6982 & Security status not satisfied \\
\hline 6983 & Authentication method blocked (ie. PIN code blocked) \\
\hline 6985 & Conditions of use not satisfied \\
\hline 6999 & Applet select failed \\
\hline $6 \mathrm{~A} 80$ & Invalid parameters in command Data Field \\
\hline $6 \mathrm{~A} 82$ & Applet or file not found \\
\hline $6 \mathrm{~A} 84$ & Insufficient memory space to complete command \\
\hline $6 \mathrm{~A} 86$ & Incorrect P1 or P2 parameter \\
\hline $6 \mathrm{~A} 88$ & Referenced data not found \\
\hline $6 \mathrm{D} 00$ & Unknown instruction given in the command \\
\hline $6 \mathrm{E} 00$ & Wrong class given in the command \\
\hline $6 \mathrm{~F} 00$ & Technical problem with no diagnostic given \\
\hline
\end{tabular}

\subsubsection{Common Virtual Machine Card Edge Interface Methods}

The common virtual machine APDUs are shown in Table 5-12.

Table 5-12: Common VM APDUs

\begin{tabular}{|l|c|c|c|c|c|c|c|}
\multicolumn{1}{|c|}{ Card Function } & CLA & INS & P1 & P2 & $\mathbf{L}_{\mathbf{c}}$ & Data & $\mathbf{L}_{\mathbf{e}}$ \\
\hline SELECT APPLET & $0 \times 00$ & 0xA4 & $0 \times 04$ & $0 \times 00$ & $\mathrm{~L}_{c}$ & AID & - \\
\hline SELECT OBJECT & $0 \times 00$ & 0xA4 & $0 \times 02$ & $0 \times 00$ & $\mathrm{~L}_{c}$ & File ID & - \\
\hline GET PROPERTIES & $0 \times 00$ & $0 \times 56$ & $\mathrm{P} 1$ & $0 \times 00$ & $\mathrm{~L}_{c}$ & Requested Tags & - \\
\hline GET ACR & $0 \times 80$ & $0 \times 4 C$ & $\mathrm{P} 1$ & $0 \times 00$ & $\mathrm{~L}_{c}$ & AID or Object ID & - \\
\hline GET RESPONSE & $0 \times 00$ & $0 \times C 0$ & $0 \times 00$ & $0 \times 00$ & - & - & $\mathrm{L}_{e}$ \\
\hline VERIFY PIN & $0 \times 00$ & $0 \times 20$ & $0 \times 00$ & $0 \times 00$ & $\mathrm{~L}_{c}$ & PIN & - \\
\hline
\end{tabular}

\subsubsection{Access Control}

A fixed set of Access Control Rules (ACR) are assigned to the Common Virtual Machine Card Edge Interface APDU commands as defined in Table 5-13: 
NIST IR 6887 - 2003 EDITION, GSC-IS VERSION 2.1

Table 5-13: ACRs assigned to the Common VM CEI

\begin{tabular}{|l|c|}
\multicolumn{1}{|c|}{ APDU } & ACR \\
\hline Get Properties & BSI_ACR_ALWAYS \\
\hline Get ACR & BSI_ACR_ALWAYS \\
\hline Get Challenge & BSI_ACR_ALWAYS \\
\hline External Authenticate & BSI_ACR_ALWAYS \\
\hline Get Response & - \\
\hline Verify PIN & BSI_ACR_ALWAYS \\
\hline
\end{tabular}




\subsubsection{Select Applet APDU}

The command is used to select the instance of an applet using its AID.

Command Message

\begin{tabular}{|l|l|}
\hline CLA & 0x00 \\
\hline INS & 0xA4 \\
\hline P1 & 0x04 \\
\hline P2 & 0x00 \\
\hline LC & Length of the applet AID \\
\hline Data Field & Applet AID (between 5 and 16 bytes in length). \\
\hline Le & Empty \\
\hline
\end{tabular}

\section{Response Message}

Data field returned in the response message

Empty.

Processing state returned in the response message

If the applet is not found on the smart card, the ISO 7816-4 [ISO4] status condition: '6A82' is returned (status bytes $\mathrm{SW} 1, \mathrm{SW} 2=0 \times 6 \mathrm{~A}, 0 \mathrm{x} 82$ ). For other status conditions see section General Error Conditions in Section 5.3.2. 


\subsubsection{Select Object APDU}

The command is used to select a container managed by an applet.

\section{Command Message}

\begin{tabular}{|l|l|}
\hline CLA & 0x00 \\
\hline INS & 0xA4 \\
\hline P1 & 0x02 \\
\hline P2 & 0x00 \\
\hline$L_{c}$ & Length of the object ID, 2 bytes. \\
\hline Data Field & Object ID. \\
\hline$L_{e}$ & Empty \\
\hline
\end{tabular}

\section{Response Message}

Data field returned in the response message

Empty.

\section{Status bytes returned in the response message}

If the object is not found, the ISO 7816-4 [ISO4] status condition: '6A82' is returned (status bytes $\mathrm{SW} 1=0 \mathrm{x} 6 \mathrm{~A}, \mathrm{SW} 2=0 \mathrm{x} 82$ ). For other status conditions see section General Error Conditions in Section 5.3.2. 


\subsubsection{Get Properties APDU}

This command is used to retrieve applet instance properties of a currently selected applet.

\section{Command Message}

\begin{tabular}{|l|l|}
\hline CLA & 0x00 \\
\hline INS & 0x56 \\
\hline P1 & Requested properties information type \\
\hline P2 & 0x00 \\
\hline LC & If $\mathrm{P} 1=0 \times 02$ then length of list of requested tags, else empty. \\
\hline Data Field & If $\mathrm{P} 1=0 \times 02$ then list of requested tags, else empty. \\
\hline Le & Expected applet instance properties length \\
\hline
\end{tabular}

\section{Reference control parameter P1}

The reference control parameter P1 shall be used to indicate the type of requested properties information. The following P1 values are possible:

0x00: Get a GSC-IS v2.0 compatible properties response message. If this response cannot be supported by the smart card then an error (0x6A86) shall be returned.

0x01: Get all the properties.

0x02: Get the properties of the tags provided in list of tags in the command data field.

\section{Data field sent in the command message}

This field is present only when P1 is $0 \mathrm{x} 02$. In that case, this data field is composed of the list of tags to be requested from the applet instance (the tag values, 1 byte each, are chained).

\section{Response Message}

\section{Data field returned in the response message when $\mathrm{P1}$ is $0 \times 00$}

The Data field returned in the response message contains the values of the following properties:

- Applet family (1 byte)

- Applet version (4 bytes)

- RFU byte

- RFU byte

- ID/CHV-applet AID length (1 byte)

- ID/CHV-applet AID (always 16 bytes padded with 0 if necessary) - AID of the ID/CHV applet instance that shall be used for Card Holder Verification (CHV) 
- Key Set Version (1 byte) 1

- Key Set Id (1 byte) 2

- T-Buffer length (2 bytes)

- V-Buffer length (2 bytes)

- X bytes of applet specific information and RFU to complement to 64 bytes.

\section{Data field returned in the response message when $\mathrm{P1}$ is $0 \mathrm{x01}$ or $0 \mathrm{x} 02$}

The data field returned in the response message contains the current value of all the properties when $\mathrm{P} 1$ is $0 \mathrm{x} 01$ or the current value of the requested properties when $\mathrm{P} 1$ is $0 \mathrm{x} 02$. The properties are returned in a single buffer containing a list of TLVs packed end-to-end according to the table below. The scope of these tags is specific to the properties object and should not be confused with the GSC and CAC data model tags.

\begin{tabular}{|c|c|c|}
\hline Tag & Length & Value \\
\hline \multirow{3}{*}{$0 \times 01$} & \multirow{3}{*}{5} & Applet Information \\
\hline & & Applet Family ( 1 byte) \\
\hline & & Applet version (4 bytes) \\
\hline $0 \times 40$ & 1 & Number of objects managed by this instance \\
\hline $0 \times 50$ & 11 & First TV-Buffer Object \\
\hline $0 \times 41$ & 2 & ObjectID (2 bytes) \\
\hline \multirow{5}{*}{$0 \times 42$} & \multirow{5}{*}{5} & Buffer Properties ( 5 bytes) \\
\hline & & Type of Tag Supported (1 byte) \\
\hline & & T-Buffer length ( 2 bytes): LSB, MSB \\
\hline & & V-Buffer length ( 2 bytes): LSB, MSB \\
\hline & & (Next TV-Buffer Object...) \\
\hline $0 \times 50$ & 11 & Last TV-Buffer Object \\
\hline $0 \times 41$ & 2 & ObjectID (2 bytes) \\
\hline \multirow{4}{*}{$0 \times 42$} & \multirow{4}{*}{5} & Buffer Properties ( 5 bytes) \\
\hline & & Type of Tag Supported (1 byte) \\
\hline & & T-Buffer length ( 2 bytes): LSB, MSB \\
\hline & & V-Buffer length ( 2 bytes): LSB, MSB \\
\hline $0 \times 51$ & 17 & First PKI Object \\
\hline $0 \times 41$ & 2 & ObjectID (2 bytes) \\
\hline \multirow{4}{*}{$0 \times 42$} & \multirow{4}{*}{5} & Buffer Properties \\
\hline & & Type of Tag Supported (1 byte) \\
\hline & & T-Buffer length (2 bytes): LSB, MSB \\
\hline & & V-Buffer length (2 bytes): LSB, MSB \\
\hline \multirow[t]{2}{*}{$0 \times 43$} & \multirow[t]{2}{*}{4} & PKI Properties \\
\hline & & Algorithm ID (1 byte) \\
\hline
\end{tabular}

${ }^{1}$ Key Set and Key Levels are applicable to v2.0 for backward compatibility.

${ }^{2}$ Key Set ID refers to the key number and the Key Level is used to indicate whether the referenced key is part of the READ or WRITE Key Set. 


\begin{tabular}{|c|c|c|}
\hline Tag & Length & Value \\
\hline & & Key Length Bytes / 8 (1024 bits -> 128 bytes-> 0x10) ( 1 byte) \\
\hline & & Private Key Initialized ( 1 byte) \\
\hline & & Public Key Initialized (1 byte) \\
\hline
\end{tabular}

\section{Processing state returned in the response message}

If the properties retrieval succeeds, SW1 $=0 \times 61$ and SW2 $=$ size of next block of data available to read.

If $\mathrm{P} 1=0 \mathrm{x} 00$ cannot be supported by the smart card, SW1 = 0x6A and SW2 $=86$.

\begin{tabular}{|c|c|l|}
\hline SW1 & SW2 & \multicolumn{1}{c|}{ Meaning } \\
\hline 61 & LL & More data available, 0xLL specifying the size of next block to read. \\
\hline $6 \mathrm{~A}$ & 86 & P1 or P2 parameter not supported. \\
\hline
\end{tabular}

For other status conditions see Table 5-11b. 


\subsubsection{Get ACR APDU}

This command is used to retrieve Access Control Rule properties.

\section{Command Message}

\begin{tabular}{|c|c|}
\hline CLA & $0 \times 80$ \\
\hline INS & $0 \times 4 C$ \\
\hline P1 & Reference Control Parameter P1 \\
\hline P2 & $0 \times 00$ \\
\hline Lc & $\begin{array}{l}\text { If } P 1=0 \times 00,0 \times 10,0 \times 20 \text {, or } 0 \times 21 \text { then empty. If } P 1=0 \times 01 \text { then the } \\
\text { length of the } A C R I D(0 \times 01) \text {. If } P 1=0 \times 11 \text { then the length of the AID } \\
(<=0 \times 10) \text {. If } P 1=0 \times 12 \text { then the length of object ID }(0 \times 02)\end{array}$ \\
\hline Data Field & $\begin{array}{l}\text { If } P 1=0 \times 00,0 \times 10,0 \times 20,0 \times 21 \text { then empty. If } P 1=0 \times 01 \text { then the } \\
\text { value of the ACRID. If } P 1=0 \times 11 \text { then the value of the AID. If } \\
P 1=0 \times 12 \text { then the value of the object ID. }\end{array}$ \\
\hline Le & Empty. \\
\hline
\end{tabular}

\section{Reference control parameter P1}

The reference control parameter P1 shall be used to indicate the type of requested ACR properties information. The following P1 values are possible:

0x00: All ACR table entries are to be extracted.

0x01: Only one entry of the ACR table is extracted based on ACRID.

0x10: All Applet/Object ACR table entries are to be extracted.

0x11: Only the entries of the Applet/Object ACR table for one applet are extracted based on applet AID.

0x12: Only one entry of the Applet/Object ACR table for an object is extracted based on object ID.

0x20: The Access Method Provider table is extracted.

0x21: The Service Applet table is extracted.

\section{Data field sent in the command message}

This field is present only when $\mathrm{P} 1$ is $0 \mathrm{x} 11$ or $0 \mathrm{x} 12$. If $\mathrm{P} 1$ equals $0 \mathrm{x} 11$, it contains the AID value of the applet for which the Applet/Object ACR table is to be extracted. If P1 equals 0x12, it contains the Object ID value of the object for which the Applet/Object ACR table is to be extracted.

\section{Response Message}

\section{Data field returned in the response message}

The following tables may be retrieved: 
- ACR table: This table maps the Access Control Rule Type (ACRType) and Access Method information to the Access Control Rule Identifier (ACRID) for each Access Control Rule.

- Applet/Object ACR table: This table maps the service (INS code/P1 byte/P2 byte/1 ${ }^{\text {st }}$ data byte) to the ACRID for each container.

- Access Method Provider table: This table maps the Access Method Provider ID to the full AID for each Access Method Provider.

- Service Applet table: This table maps the Service Applet ID to the full AID for each Service Applet.

The data fields returned in the response message may contain all the entries for a table or only the requested ones depending on the command parameters.

The following entry is always returned and precedes any ACR table, Applet/Object ACR table or Authentication Method Provider table.

Table 5-14: Applet Information String

\begin{tabular}{|c|c|c|}
\hline Tag & Length & Value \\
\hline $0 \times 01$ & 5 & Applet Family of Access Control Applet (ACA) (1 byte) \\
\cline { 2 - 3 } & & Applet version of ACA (4 bytes) \\
\hline
\end{tabular}

In addition to the common Applet Information entry the following entries are conditionally returned depending on the reference control parameter $\mathrm{P} 1$.

\section{Data field returned in the response message when P1 is 0x00}

The data field returned in the response message contains all the entries of the ACR table.

Table 5-15: ACR Table

\begin{tabular}{|c|c|c|}
\hline Tag & Length & Value \\
\hline $0 \times A 1$ & 1 & Number of ACR entries (unique ACRID) \\
\hline \multirow{9}{*}{$0 \times A 0$} & \multirow{9}{*}{ * } & First ACR entry (structured as follows) \\
\hline & & ACRID of ACR entry (1 byte) \\
\hline & & ACRType (as defined in Table 3-2) (1 byte) \\
\hline & & Number of AccessMethods in this ACR ( 1 byte) \\
\hline & & First AccessMethodProviderID (1 byte) \\
\hline & & First keyIDOrReference (1 byte) \\
\hline & & (Next AccessMethod...) \\
\hline & & Last AccessMethodProviderID (1 byte) \\
\hline & & Last keyIDOrReference (1 byte) \\
\hline $0 \times A 0$ & * & (Next ACR entry ...) \\
\hline
\end{tabular}

* Denotes Variable length field 


\section{Data field returned in the response message when P1 is 0x01}

The data field returned in the response message a single entry of the ACR table based on ACRID.

Table 5-16: Applet/Object ACR Table

\begin{tabular}{|c|c|c|}
\hline Tag & Length & Value \\
\hline \multirow{9}{*}{ OXA0 } & \multirow{9}{*}{ * } & ACR entry corresponding to ACRID sent \\
\hline & & ACRID of ACR entry ( 1 byte) \\
\hline & & ACRType (as defined in Table 3-2) (1 byte) \\
\hline & & Number of AccessMethods in this ACR ( 1 byte) \\
\hline & & First AccessMethodProviderID (1 byte) \\
\hline & & First keyIDOrReference ( 1 byte) \\
\hline & & (Next AccessMethod...) \\
\hline & & Last AccessMethodProviderID (1 byte) \\
\hline & & Last keyIDOrReference ( 1 byte) \\
\hline
\end{tabular}

* Denotes Variable length field

\section{Data field returned in the response message when $\mathrm{P1}$ is $0 \times 10$}

The data field returned in the response message contains all entries of the Applet/Object ACR table.

Table 5-17: Access Method Provider Table

\begin{tabular}{|c|c|c|}
\hline Tag & Length & Value \\
\hline $0 \times 81$ & 1 & Number of applets managed by this ACA \\
\hline \multirow[t]{3}{*}{$0 \times 80$} & \multirow{3}{*}{$\begin{array}{l}\text { Length is } 2 \\
\text { plus length } \\
\text { of nested } \\
\text { TLV fields } \\
0 \times 82\end{array}$} & Card Applet ACR structured as follows \\
\hline & & Applet ID (1 byte) \\
\hline & & Number of objects managed by this applet ( 1 byte) \\
\hline \multirow{12}{*}{$0 \times 82$} & \multirow{12}{*}{ * } & Card Object ACR structured as follows \\
\hline & & Card Object ID (2 bytes) \\
\hline & & INS1 Code (1 byte) \\
\hline & & INS1 Configuration Definition - $00000 b_{2} b_{1} b_{0}(1$ byte $)$ \\
\hline & & If $\mathrm{b}_{0}=1$ then $\mathrm{P} 1$ byte is present. \\
\hline & & If $b_{1}=1$ then $P 2$ byte is present. \\
\hline & & If $b_{2}=1$ then first data field byte is present. \\
\hline & & P1 Value - OPTIONAL (1 byte) \\
\hline & & P2 Value - OPTIONAL (1 byte) \\
\hline & & First Data Byte Value - OPTIONAL (1 byte) \\
\hline & & ACRID (1 byte) \\
\hline & & INSx ... \\
\hline $0 \times 82$ & * & (Next Card Object ACR...) \\
\hline $0 \times 80$ & * & (Next Card Applet ACR...) \\
\hline
\end{tabular}


* Denotes Variable length field

\section{Data field returned in the response message when P1 is 0x11}

The data field returned in the response message contains the entries of the Applet/Object ACR table for a single applet based on AID. 
Table 5-18: Service Applet Table

\begin{tabular}{|c|c|c|}
\hline Tag & Length & Value \\
\hline \multirow{3}{*}{$0 \times 80$} & \multirow{3}{*}{$\begin{array}{c}\text { Length is } 2 \\
\text { plus length } \\
\text { of nested } \\
\text { TLV fields } \\
0 \times 82\end{array}$} & Applet ACR table based on applet AID entered \\
\hline & & Applet ID (1 byte) \\
\hline & & Number of objects managed by this applet ( 1 byte) \\
\hline \multirow{12}{*}{$0 \times 82$} & \multirow{12}{*}{ 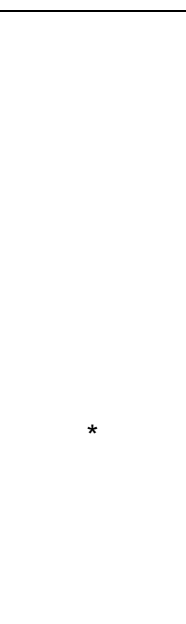 } & Card Object ACR structured as follows \\
\hline & & Card Object ID (2 bytes) \\
\hline & & INS1 Code (1 byte) \\
\hline & & INS1 Configuration Definition - $00000 b_{2} b_{1} b_{0}(1$ byte $)$ \\
\hline & & If $b_{0}=1$ then $P 1$ byte is present. \\
\hline & & If $b_{1}=1$ then $P 2$ byte is present. \\
\hline & & If $b_{2}=1$ then first data field byte is present. \\
\hline & & P1 Value - OPTIONAL (1 byte) \\
\hline & & P2 Value - OPTIONAL (1 byte) \\
\hline & & First Data Byte Value - OPTIONAL (1 byte) \\
\hline & & ACRID (1 byte) \\
\hline & & $(I N S x \ldots)$ \\
\hline $0 \times 82$ & * & (Next Card Object ACR...) \\
\hline
\end{tabular}

* Denotes Variable length field

\section{Data field returned in the response message when $\mathrm{P1}$ is $0 \times 12$}

The data field returned in the response message contains the entry of the Applet/Object ACR table for a single object based on OID.

Table 5-19: Applet/Object ACR table for a Single Object

\begin{tabular}{|c|c|c|}
\hline Tag & Length & Value \\
\hline \multirow{12}{*}{$0 \times 82$} & \multirow{12}{*}{ * } & Card Object ACR (structured as follows) \\
\hline & & Card Object ID (2 bytes) \\
\hline & & INS1 Code (1 byte) \\
\hline & & INS1 Configuration Definition - $00000 b_{2} b_{1} b_{0}(1$ byte $)$ \\
\hline & & If $\mathrm{b}_{0}=1$ then $\mathrm{P} 1$ byte is present. \\
\hline & & If $b_{1}=1$ then $P 2$ byte is present. \\
\hline & & If $b_{2}=1$ then first data field byte is present. \\
\hline & & P1 Value - OPTIONAL (1 byte) \\
\hline & & P2 Value - OPTIONAL (1 byte) \\
\hline & & First Data Byte Value - OPTIONAL (1 byte) \\
\hline & & ACRID (1 byte) \\
\hline & & $($ INSx ...) \\
\hline
\end{tabular}

* Denotes Variable length field 


\section{Data field returned in the response message when $P 1$ is $0 \times 20$}

The data field returned in the response message contains all the entries of the Access Method Provider table.

Table 5-20: Access Method Provider Table

\begin{tabular}{|c|c|l|}
\hline Tag & Length & \multicolumn{1}{c|}{ Value } \\
\hline $0 \times 91$ & 1 & Number of AMP entries \\
\hline $0 \times 90$ & $\begin{array}{c}\text { Length } \\
\text { includes } \\
\text { nested TLV } \\
\text { structure } \\
0 \times 92\end{array}$ & AMP entry (structured as follows) \\
\hline & $*$ & Access Method provider AID \\
\hline $0 \times 92$ & $*$ & (Next AMP entry...) \\
\hline $0 \times 90$ & & \\
\hline
\end{tabular}

\section{Data field returned in the response message when $\mathrm{P1}$ is $0 \times 21$}

The data field returned in the response message contains all the entries of the Service Applet table.

Table 5-21: Service Applet Table

\begin{tabular}{|c|c|c|}
\hline Tag & Length & Value \\
\hline $0 \times 94$ & 1 & Number of Applet entries \\
\hline \multirow[t]{2}{*}{$0 \times 93$} & \multirow[t]{2}{*}{ * } & Applet entry (structured as follows) \\
\hline & & Applet ID (short form) (1 byte) \\
\hline $0 \times 92$ & * & Applet AID \\
\hline $0 \times 93$ & * & (Next Applet entry) \\
\hline
\end{tabular}

* Denotes Variable length field

Processing state returned in the response message

If properties retrieval succeeds, SW1 = 0x61 and SW2 = size of next block of data available to read.

\begin{tabular}{|c|c|l|}
\hline SW1 & SW2 & Meaning \\
\hline 61 & LL & More data available, 0xLL specifying the size of next block to read. \\
\hline
\end{tabular}

For other status conditions see Table 5-11b. 


\subsubsection{Get Response APDU}

The GET RESPONSE APDU is used to retrieve from the smart card the response message of the immediately preceding APDU in the case that this APDU has returned a processing state of $61 \mathrm{xx}$ indicating that a response message of $\mathrm{xx}$ bytes is available.

\section{Command Message}

\begin{tabular}{|l|l|}
\hline CLA & $0 \times 00$ \\
\hline INS & $0 \times C 0$ \\
\hline P1 & $0 \times 00$ \\
\hline P2 & $0 \times 00$ \\
\hline $\mathbf{L}_{c}$ & Empty \\
\hline Data Field & Empty \\
\hline $\mathbf{L}_{e}$ & Number of bytes to read in response \\
\hline
\end{tabular}

\section{Response Message}

Data field returned in the response message

If the APDU result indicates success, $\mathrm{L}_{\mathrm{e}}$ number of bytes will be available to read from the smart card.

Processing state returned in the response message

See Table 5-11b. 


\subsubsection{Verify PIN APDU}

The VERIFY command is used to verify the global PIN code, or to check if the PIN code verification is required, or to check whether or not the PIN code has been already verified. The global PIN is a root level key.

\section{Command Message}

\begin{tabular}{|l|l|}
\hline CLA & 0x00 \\
\hline INS & 0x20 \\
\hline P1 & $0 \times 00$ \\
\hline P2 & 0x00 \\
\hline Lc & 0xNN (Effective PIN length, 0x00 indicates no PIN present) \\
\hline Data Field & PIN code to be verified \\
\hline Le & Empty \\
\hline
\end{tabular}

Note: The maximum effective PIN length is dependent on the card platform.

\section{Data field sent in the command message}

If the data length and the data field sent in the command message are empty (data field does not include a PIN code), the command corresponds to a PIN verify check command, and it is used to determine if the PIN code verification is necessary and whether or not the PIN code has been already verified.

If the verification fails, the PIN-tries-remaining flag is decremented, and the PIN-verified flag value does not change. The PIN-always flag value is set to 0x00. If the PIN-tries-remaining flag value is $0 \mathrm{x} 00$, the PIN code is considered blocked. If the verification succeeds, the PIN-verified flag value and the PIN-always flag value are both set to $0 \mathrm{x} 01$.

\section{Response Message}

\section{Data field returned in the response message}

The data field in the response message is always empty.

Processing state returned in the response message

If PIN verification succeeds, $\mathrm{SW} 1=0 \times 90$ and $\mathrm{SW} 2=0 \mathrm{x} 00$.

If $\mathrm{PIN}$ verification fails, the status returned is $\mathrm{SW} 1=0 \mathrm{x} 63, \mathrm{SW} 2=0 \mathrm{xCX}$ where $\mathrm{X}$ is number of remaining PIN tries.

If PIN verify check command is submitted and PIN is already verified, SW $1=0 \times 90$ and $\mathrm{SW} 2=0 \mathrm{x} 00$, otherwise $\mathrm{SW} 1=0 \mathrm{x} 63, \mathrm{SW} 2=0 \mathrm{xCX}$, where $\mathrm{X}=$ number of remaining PIN tries. 
NIST IR 6887 - 2003 EDITION, GSC-IS VERSION 2.1

\begin{tabular}{|c|c|l|}
\hline SW1 & SW2 & \multicolumn{1}{|c|}{ Meaning } \\
\hline 90 & 00 & PIN verification succeeds \\
\hline 63 & CX & PIN not verified and X indicates the remaining tries \\
\hline 69 & 83 & PIN code blocked \\
\hline $6 \mathrm{~A}$ & 88 & No PIN code defined \\
\hline
\end{tabular}




\subsubsection{Generic Container Provider Virtual Machine Card Edge Interface}

Table 5-22 shows the Generic Container Provider VM APDUs. As described in Chapter 8, containers accessed by these APDUs are split into two buffers: a TL buffer containing Tag and associated Length values, and a $\mathrm{V}$ buffer containing the values identified by the corresponding Tags and Lengths.

Table 5-22: Generic Container VM APDUs

\begin{tabular}{|l|c|c|c|c|c|c|c|}
\hline \multicolumn{1}{|c|}{ Card Function } & CLA & INS & P1 & P2 & $\mathbf{L}_{\mathbf{c}}$ & Data & $\mathbf{L}_{\mathbf{e}}$ \\
\hline READ BUFFER & $0 \times 80$ & $0 \times 52$ & Off/H & Off/L & $0 \times 02$ & $\begin{array}{c}\text { Buffer and } \\
\text { number bytes to } \\
\text { read }\end{array}$ & - \\
\hline UPDATE BUFFER & $\begin{array}{c}0 \times 80, \\
0 \times 84\end{array}$ & $0 \times 58$ & Off/H & Off/L & $L_{c}$ & $\begin{array}{c}\text { Buffer and data } \\
\text { to update }\end{array}$ & - \\
\hline
\end{tabular}




\subsubsection{Update Buffer APDU}

This command allows updating all or part of a buffer.

\section{Command Message}

\begin{tabular}{|l|l|}
\hline CLA & 0x80 \\
\hline INS & 0x58 \\
\hline P1 & Reference Control Parameter P1 \\
\hline P2 & Reference Control Parameter P2 \\
\hline Lc & 1+ Length of data to be updated \\
\hline Data Field & Buffer (1 byte) + data to be updated \\
\hline Le & Empty \\
\hline
\end{tabular}

\section{Reference control parameter P1/P2}

The reference control parameters $\mathrm{P} 1$ and $\mathrm{P} 2$ shall be used to store the offset from which data are to be written. This offset is calculated by concatenating the $\mathrm{P} 1$ and $\mathrm{P} 2$ parameters $(\mathrm{P} 1=\mathrm{MSB}, \mathrm{P} 2$ $=$ LSB).

\section{Data field sent in the command message}

The first byte of the data field shall be used to indicate which buffer is to be updated.

The possible values are:

0x01: $\quad$ T-buffer

0x02: V-buffer

The other bytes correspond to the data to be updated.

\section{Response Message}

\section{Data field returned in the response message}

The data field in the response message is always empty.

Processing state returned in the response message

\begin{tabular}{|c|c|l|}
\hline SW1 & SW2 & \multicolumn{1}{|c|}{ Meaning } \\
\hline 67 & 00 & Invalid command data length \\
\hline $6 \mathrm{~A}$ & 86 & Wrong P1/P2 (Try to update data out of the buffer) \\
\hline $6 \mathrm{~A}$ & 88 & No corresponding buffer (invalid Buffer Type) \\
\hline
\end{tabular}




\subsubsection{Read Buffer APDU}

This command allows reading all or part of a buffer.

\section{Command Message}

\begin{tabular}{|l|l|}
\hline CLA & 0x80 \\
\hline INS & 0x52 \\
\hline P1 & Reference Control Parameter P1 \\
\hline P2 & Reference Control Parameter P2 \\
\hline LC & 0x01 + 0x01 = 0x02 \\
\hline Data Field & $\begin{array}{l}\text { Buffer type (1 byte value) followed by the data length to read (1 byte } \\
\text { value) }\end{array}$ \\
\hline Le & Empty \\
\hline
\end{tabular}

\section{Reference control parameter P1/P2}

The reference control parameters P1 and P2 shall be used to store the offset from which data are to be read. This offset is calculated by concatenating the $\mathrm{P} 1$ and $\mathrm{P} 2$ parameters $(\mathrm{P} 1=\mathrm{MSB}, \mathrm{P} 2=$ LSB).

\section{Data field sent in the command message}

The data field shall be used to indicate which buffer is to be read.

The possible values are:

0x01: T-buffer

0x02: V-buffer

\section{Response Message}

\section{Data field returned in the response message}

The data field in the response message corresponds to the data read from the smart card, according to the P1, P2 parameters (offset indicating from where to read data) or empty if GET RESPONSE command is required to receive data read from the smart card.

\section{Processing state returned in the response message}

If READ BUFFER command was successful, $S W 1=0 \times 90$ and $S W 2=0 x 00$, any available data is returned in the data field of the response message. If command is successful and SW1=0x61, SW2 contains bytes remaining to be read from the smart card with subsequent GET RESPONSE commands.

\begin{tabular}{|c|c|l|}
\hline SW1 & SW2 & \multicolumn{1}{|c|}{ Meaning } \\
\hline 67 & 00 & Invalid command data length \\
\hline $6 \mathrm{~A}$ & 86 & Wrong P1/P2 (Try to update data out of the buffer) \\
\hline $6 \mathrm{~A}$ & 88 & No corresponding buffer (invalid Buffer Type) \\
\hline
\end{tabular}




\subsubsection{Symmetric Key Provider Virtual Machine Card Edge Interface}

Table 5-23 shows the Symmetric Key Provider VM APDUs.

Table 5-23: Symmetric Key VM APDUs

\begin{tabular}{|l|c|c|c|c|c|c|c|}
\hline \multicolumn{1}{|c|}{ Card Function } & CLA & INS & P1 & P2 & $\mathrm{L}_{c}$ & Data & $\mathrm{L}_{\mathbf{e}}$ \\
\hline GET CHALLENGE & $0 \times 00$ & $0 \times 84$ & $0 \times 00$ & $0 \times 00$ & - & - & $\mathrm{L}_{e}$ \\
\hline $\begin{array}{l}\text { EXTERNAL } \\
\text { AUTHENTICATE }\end{array}$ & $0 \times 00$ & $0 \times 82$ & AlgID & Key \# & $\mathrm{L}_{c}$ & Cryptogram & - \\
\hline $\begin{array}{l}\text { INTERNAL } \\
\text { AUTHENTICATE }\end{array}$ & $0 \times 00$ & $0 \times 88$ & AlgID & Key \# & $\mathrm{L}_{c}$ & Challenge & $\mathrm{L}_{e}$ \\
\hline
\end{tabular}

\subsubsection{Get Challenge APDU}

The GET CHALLENGE command is the first step of the host authentication process and is followed immediately by the EXTERNAL AUTHENTICATE command. The computed challenge is valid only for the following EXTERNAL AUTHENTICATE APDU.

\section{Command Message}

\begin{tabular}{|l|l|}
\hline CLA & 0x00 \\
\hline INS & 0x84 \\
\hline P1 & 0x00 \\
\hline P2 & 0x00 \\
\hline LC & Empty \\
\hline Data Field & Empty \\
\hline Le & Challenge length \\
\hline
\end{tabular}

\section{Response Message}

\section{Data field returned in the response message}

The response message contains the challenge used later for authentication.

\section{Processing state returned in the response message}

See Table 5-11b.

Note: The computed challenge must be stored within the applet instance in order to evaluate the expected EXTERNAL AUTHENTICATE command. The client application shall encrypt the challenge received from the smart card using a cryptographic algorithm known by the smart card and the corresponding shared key. The cryptographic algorithm is DES3-ECB with a 16-byte key. The encrypted challenge shall then be submitted to the smart card using the EXTERNAL AUTHENTICATE command. 


\subsubsection{External Authenticate APDU}

This EXTERNAL AUTHENTICATE command is a subset of the ISO 7816-4 [ISO4] standard command. The default cryptographic algorithm is DES3-ECB with double length key size (16 bytes) and an 8-byte challenge requested from the smart card using the GET CHALLENGE command just before the authentication command is submitted. This command is introduced to allow external authentication with different cryptographic algorithms selected through the P1 parameter and multiple key sets if same data is updated by different applications that do not desire to share their keys.

\section{Command Message}

\begin{tabular}{|l|l|}
\hline CLA & 0x00 \\
\hline INS & 0x82 \\
\hline P1 & Algorithm identifier and security level \\
\hline P2 & 0x00 for default key, 0x01 to 0x30 for key number \\
\hline $\mathbf{L}_{c}$ & Length of the cryptogram \\
\hline Data Field & Cryptogram \\
\hline $\mathbf{L}_{e}$ & Empty \\
\hline
\end{tabular}

P1: $\quad$ 0xAS where A specifies the algorithm identifier using the 4-MSb of $\mathbf{P 1}$ and $\mathbf{S}$ defines the secure messaging and command encryption as described in the table below, using the 4-LSb of the parameter

$\underline{\text { Table 5-6 }}$ contains the algorithm identifiers.

\begin{tabular}{|l|l|l|l|l|l|l|l|l|l|}
\multicolumn{7}{|c|}{ P1 } & \multicolumn{1}{c|}{ Meaning of } \\
\hline b8 & b7 & B6 & b5 & b4 & b3 & b2 & b1 & \multicolumn{1}{|c|}{ A (b8 b5) } & \multicolumn{1}{|c|}{ S(b4-b1) } \\
\hline 0 & 0 & 0 & 0 & 0 & 0 & 0 & 0 & $\begin{array}{l}\text { Default algorithm or } \\
\text { already known }\end{array}$ & $\begin{array}{l}\text { No secure } \\
\text { messaging expected }\end{array}$ \\
\hline 0 & 0 & 0 & 0 & 0 & 0 & 0 & 1 & $\begin{array}{l}\text { Default algorithm or } \\
\text { already known }\end{array}$ & $\begin{array}{l}\text { Secure messaging } \\
\text { C-MAC (Global } \\
\text { Platform) }\end{array}$ \\
\hline 0 & 0 & 0 & 0 & 0 & 0 & 1 & 1 & $\begin{array}{l}\text { Default algorithm or } \\
\text { already known }\end{array}$ & $\begin{array}{l}\text { Command } \\
\text { encryption and C- } \\
\text { MAC (Global } \\
\text { Platform) }\end{array}$ \\
\hline- & - & - & - & 0 & 0 & 0 & 0 & Algorithm Identifier & $\begin{array}{l}\text { No secure } \\
\text { messaging expected }\end{array}$ \\
\hline- & - & - & - & 0 & 0 & 0 & 1 & Algorithm Identifier & $\begin{array}{l}\text { Secure messaging } \\
\text { C-MAC (Global } \\
\text { Platform) }\end{array}$ \\
\hline- & - & - & - & 0 & 0 & 1 & 1 & Algorithm Identifier & $\begin{array}{l}\text { Command } \\
\text { encryption and C- } \\
\text { MAC (Global } \\
\text { Platform) }\end{array}$ \\
\hline
\end{tabular}

\section{Response Message}


NIST IR 6887 - 2003 EDITION, GSC-IS VERSION 2.1

\section{Data field returned in the response message}

Empty.

Processing state returned in the response message:

For specific status conditions see Table 5-11b. 


\subsubsection{Internal Authenticate APDU}

This command is used to perform a challenge-response authentication.

\section{Command Message}

\begin{tabular}{|l|l|}
\hline CLA & 0x00 \\
\hline INS & 0x88 \\
\hline P1 & $\begin{array}{l}\text { 0x00 for the default DES3-ECB or Algorithm ID as defined in the } \\
\text { CCC }\end{array}$ \\
\hline P2 & 0x00 for default key, 0x01 to 0x30 for key number \\
\hline Lc & Length of the subsequent data field \\
\hline Data Field & Authentication related data (e.g. Challenge) \\
\hline Le & 0xLL Maximum number of bytes expected in response \\
\hline
\end{tabular}

\section{Data field sent in the command message}

The data field contains the data to be encrypted by the smart card using the selected key.

\section{Response Message}

\section{Data field returned in the response message}

The data field in the response message contains the data encrypted. The length of the response may vary and depends on the configuration of the applet.

\section{Processing state returned in the response message}

See Table 5-11b. 


\subsubsection{Public Key Provider Virtual Machine Card Edge Interface}

The Public Key Provider VM APDU set consists of one APDU, the PRIVATE SIGN/DECRYPT APDU as detailed in Section 5.3.6.1.

\subsubsection{Private Sign/Decrypt APDU}

This command is used to perform an RSA signature or data decryption.

\section{Command Message}

\begin{tabular}{|l|l|}
\hline CLA & 0x80 \\
\hline INS & 0x42 \\
\hline P1 & 0x00 \\
\hline P2 & 0x00 \\
\hline Lc & Data Field length \\
\hline Data Field & Data to sign or decrypt \\
\hline Le & Expected length of the signature/decryption \\
\hline
\end{tabular}

\section{Data field sent in the command message}

The data field contains the data to be signed using the selected RSA key pair.

The data must be already padded before the message is sent.

\section{Response Message}

\section{Data field returned in the response message}

The data field in the response message contains the data signed or decrypted. The client application is responsible for any data padding.

Processing state returned in the response message

See Table 5-11b. 


\section{Card Capabilities Container}

\subsection{Overview}

To accommodate variations in smart card APDU set implementations, the GSC-IS defines a VCEI and a general mechanism for mapping a smart card's native APDU set to the VCEI. This mechanism is based on the GSC-IS Card Capability grammar. The differences between a smart card's APDU set and the standard APDU set defined by the VCEI are carried on the smart card in the CCC.

Each GSC-IS conformant smart card shall contain a CCC and support a standard procedure for accessing it as defined in Section 6.2. The contents of a CCC shall conform with the formal card capabilities grammar defined in this chapter.

Virtual Machine cards can be programmed to directly implement the VCEI APDU set. However, Virtual Machine cards shall still contain a CCC.

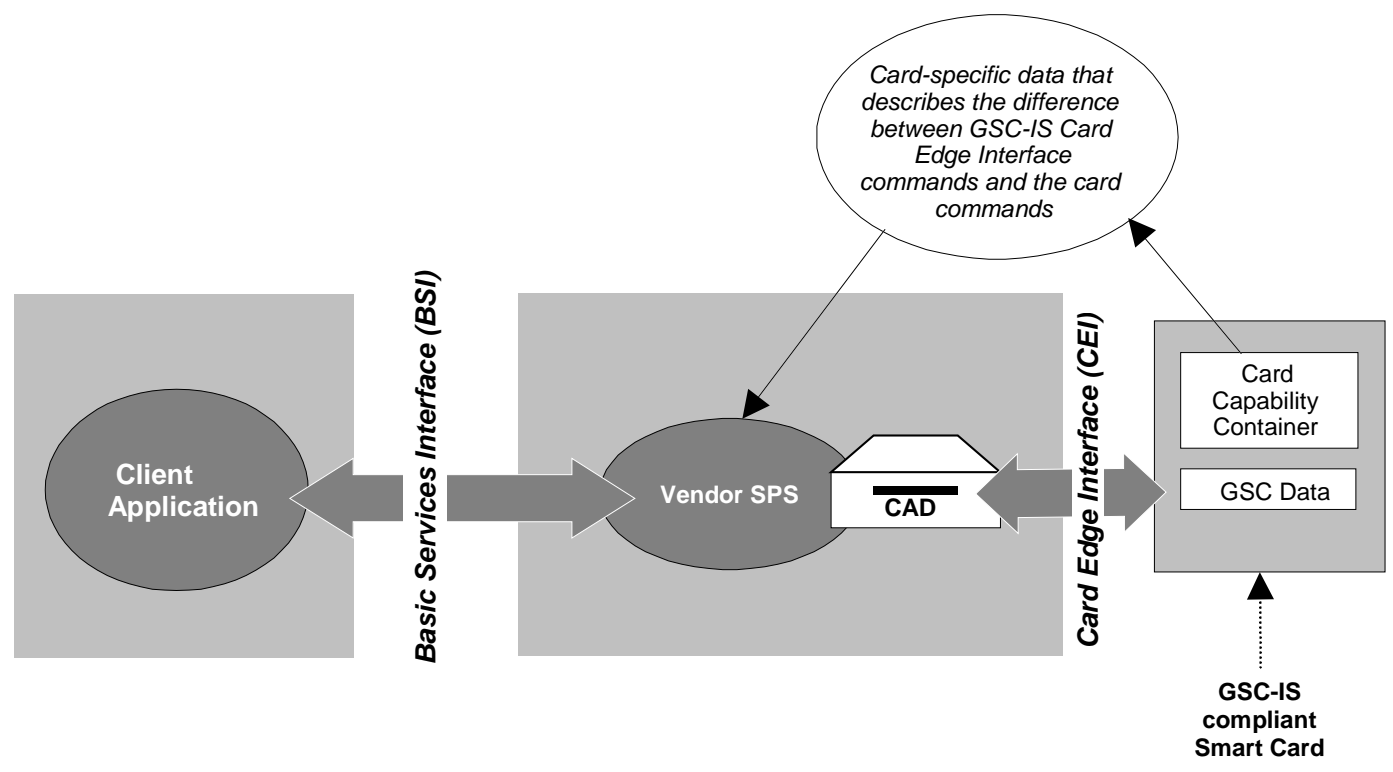

Figure 6-1: The Card Capability Container

Before the card-specific APDU definitions can be used to communicate with the smart card, the CCC must be read. 


\subsection{Procedure for Accessing the CCC}

The CCC is designated by the Capabilities Application Identifier (AID: GSC-RID\|DB00). The Universal AID of the smart card CCC shall be 0xA000000116DB00. The CCC shall be the default container of a CCC applet on a VM card. This container shall be selected by default when the CCC applet is selected.

The CCC is implemented as a transparent (binary) file on file system cards. The GSC CCC Elementary File (EF) shall be contained in the Master Directory (FID: "0x3F00") and is designated by the Capabilities Application Identifier (AID: GSC-RID\|DB00) as well as the FID: “0xDB00”.

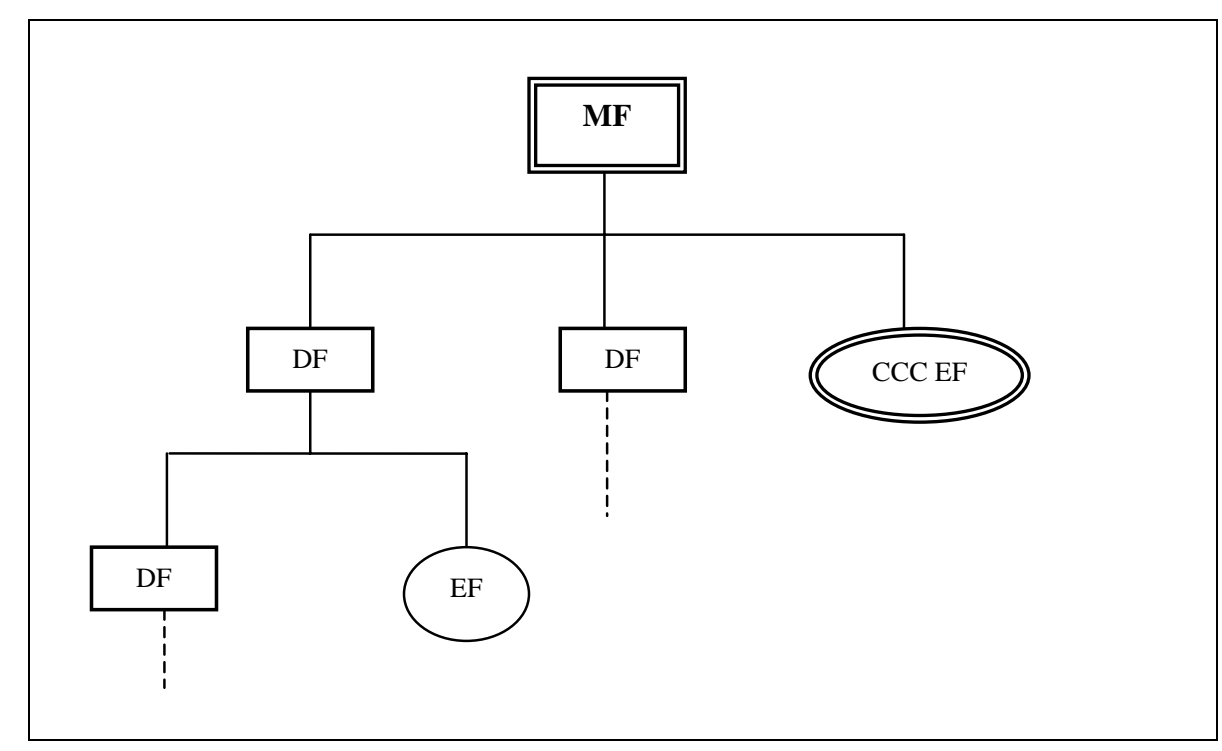

Figure 6-2: Location of the CCC Elementary File in a file system card

\subsubsection{General CCC Retrieval Sequence}

The CCC shall be stored under a known AID on Virtual Machine cards and a known FID under File system cards. The following CCC retrieval sequence shall be executed after an ATR (Answer-To-Reset) 
to the smart card. The retrieval sequence is used to determine which card edge interface is implemented, virtual machine card edge or File system card edge and then to read the CCC. Once the ATR is successful, the SPS first attempts to retrieve the CCC using the procedure for Virtual machine cards. If this fails, the SPS then attempts to read the CCC using the file system card procedure. If that also fails, the SPS assumes that the smart card does not contain a CCC and is not GSC-IS conformant.

The procedure for the retrieval of the CCC is as follows:

1. The SPS sends a SELECT APPLET APDU to the smart card as shown in the following table:

\begin{tabular}{|c|c|c|c|c|c|}
\hline CLA & INS & P1 & P2 & Lc & DATA \\
\hline $0 \times 00$ & 0xA4 & $0 \times 04$ & $0 \times 00$ & $\begin{array}{c}\text { Length } \\
\text { of AID }\end{array}$ & AID \\
\hline
\end{tabular}

2. The CCC applet is selected on a VM card if the smart card returns the status bytes "0x9000" or "0x61LL" ("LL" indicates more data available). If not, the SPS then attempts to use the File system procedure to access the CCC as defined in steps 4-8.

3. A successful applet selection is followed by an attempt to read the CCC by sending a READ BUFFER APDU command as specified in the Card Edge Interface for VM cards. The READ BUFFER APDU is sent as follows:

\begin{tabular}{|c|c|c|c|c|c|}
\hline CLA & INS & P1 & P2 & Lc & DATA \\
\hline 0x80 & 0x52 & P1 & P2 & 0x02 & $\begin{array}{c}\text { Buffer type +data } \\
\text { length to read }\end{array}$ \\
\hline
\end{tabular}

Note 1 : Reference Control Parameter P1/P2 : See Card Edge interface for VM (hapter 5 , Section 5-43)

Note 2 : The first buffer to be read is the TL-Buffer (Buffer type = "0x01"), the second buffer to be read is the V-Buffer (Buffer type = "0x02).

Note 3 : The "data length to read" is application/vendor specific, but in practice it is advisable to set it to 64 .

If no error status bytes are returned, the smart card will return the data read from the card with "0x9000" status byte to indicate complete completion or "0x61LL" to indicate that "LL" bytes are still available to read. The TL-Buffer and the V-Buffer shall be entirely read.

If an error status byte is returned and the card does not support the READ BUFFER APDU command, the SPS attempts to use the File system card edge by sending a READ BINARY APDU with CLA="0x00" as defined in step 5. If this succeeds, the VM card is using the File system card edge APDUs. If this fails and the smart card does not support READ BINARY either, the smart card is not GSC-IS compliant.

4. For the file system card, the SPS sends a sequence of APDUs to the smart card until the CCC is successfully read. This sequence selects the Master File (MF) using its reserved FID value "0x3F00", then the CCC Elementary File (EF) using its reserved FID value "0xDB00", and then performs a binary read operation on that CCC Elementary File. 
The SPS sends a SELECT MF APDU command as follows:

\begin{tabular}{|c|c|c|c|c|c|}
\hline CLA & INS & P1 & P2 & LC & DATA \\
\hline TEST CLA & $0 \times A 4$ & $0 \times 03$ & $0 \times 00$ & $0 \times 02$ & $0 \times 3 F 00$ \\
\hline
\end{tabular}

Note 1 : The default TEST CLA values are: 0x00, 0xC0, 0xF0, 0x80, 0xBC, 0x01. The CLA value "0x00" is ISO 7816-4 [ISO4] conformant. The value "0x00" shall be the first to be tested. (Additional test values for CLA are: 0x90, 0xA0, 0xB0-0xCF.)

5. If the returned status byte is "0x6E00", the tested Class byte is not supported. The SPS loops back to step 4 and attempts the next CLA value.

6. If the returned status byte is "0x9000" or "0x61LL" ("LL" indicates more data available), then the command structure and CLA value are correct.

7. Once CLA has been determined, the SPS selects (CCC) EF under MF as follows:

\begin{tabular}{|c|c|c|c|c|c|}
\hline CLA & INS & P1 & P2 & Lc & DATA \\
\hline Determined CLA & 0xA4 & 0x02 & 0x00 & 0x02 & 0xDB00 \\
\hline
\end{tabular}

The CCC EF is selected if no error codes are returned.

8. Then to Read a binary file (with no secure messaging), the SPS uses the following READ BINARY APDU on the selected CCC EF:

\begin{tabular}{|c|c|c|c|c|}
\hline CLA & INS & P1 & P2 & Le \\
\hline Determined CLA & OxB0 & Off/H & Off/L & Le \\
\hline
\end{tabular}

Note 2: P1, P2 and Le are as defined in Section 5.1.1.2

Note 3: SPS implementations should define a timeout value to avoid an infinite wait for a response from the smart card. The timeout mechanism and value are application specific, since in some cases the card reader driver layer may provide this. The SPS will return BSI_TIMEOUT_ERROR in response to a gscBsiUtilconnect () if a connection cannot be established before the timeout value expires.

\subsubsection{Card Capabilities Container Structure}

For a file system card, the Card Capability Container shall be an elementary file. The file consists of a string of SIMPLE Tag-Length-Value (TLV) data objects with no encoding, with the exception of fields that use structured SIMPLE TLV ("Application CardURL" and "Access Control Rule Table" fields).

For a VM card, the Card Capability Container shall be the default container (buffer) managed by the CCC applet. The internal format of that CCC container is defined in Section 8.2.

For both card types, the CCC is configured for ALWAYS READ. However, it is up to each implementer to define write/modify rules. 
Table 6-1: ccc Fields

\begin{tabular}{|c|c|c|c|}
\hline \multicolumn{2}{|l|}{ Card Capabilities Container } & FID: 0xDB00 & Always Read \\
\hline Data Element (TLV) & Tag & \multicolumn{2}{|c|}{ Type } \\
\hline Card Identifier & 0xF0 & Variable & \\
\hline Capability Container version number & 0xF1 & Fixed: 1 byte & \\
\hline Capability Grammar version number & $0 x F 2$ & Fixed: 1 byte & \\
\hline Applications CardURL & $0 x F 3$ & Variable - Multip & \\
\hline PKCS\#15 & 0xF4 & Fixed: 1 byte & \\
\hline Registered Data Model number & $0 x F 5$ & Fixed: 1 byte & \\
\hline Access Control Rule Table & $0 x F 6$ & Variable - Multip & \\
\hline CARD APDUs & $0 x F 7$ & Fixed: 6 bytes & \\
\hline Redirection Tag & $0 x F A$ & Variable & \\
\hline Capability Tuples (CTs) & $0 x F B$ & Variable: Collect & oyte Tuples \\
\hline Status Tuples (STs) & $0 x \mathrm{FC}$ & Variable: Collect & oyte Tuples \\
\hline Next CCC & 0xFD & Application Card & bytes or greater \\
\hline Optional Issuer Defined Objects & $\begin{array}{l}\text { Issuer } \\
\text { Defined }\end{array}$ & Variable & \\
\hline Error Detection Code & 0xFE & LRC & \\
\hline
\end{tabular}

\subsection{Fields}

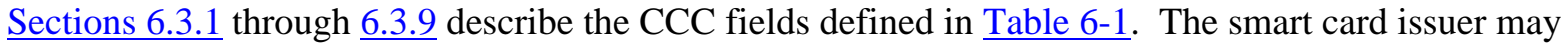
include additional TLV objects in the Card Capabilities Container for application specific purposes. These are not needed for interoperability but may be used to facilitate extended applications. They may be ignored by any implementation without affecting interoperability. Any optional objects that are not recognized shall be ignored.

\subsubsection{Card Identifier Description}

The Card Identifier shall be specified by each issuing organization for each card type. Among other things, the Card Identifier allows a client application to determine the type of card it is communicating with. This identifer is defined by the following ASN.1 sequence:

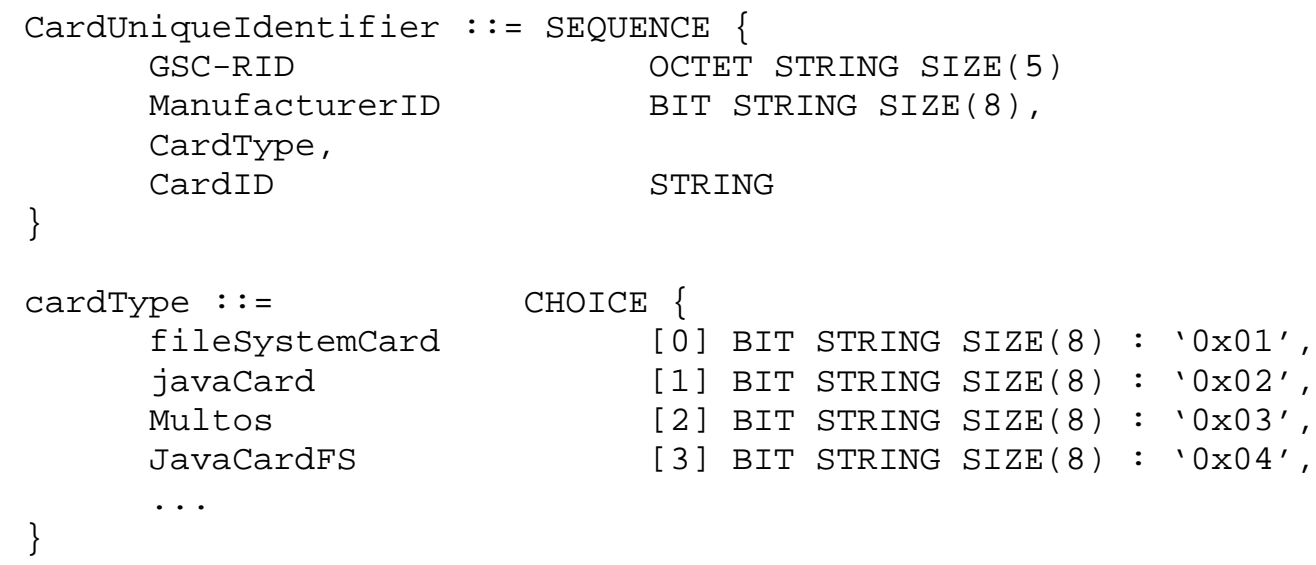




\subsubsection{Capability Container Version Number}

The Capability Container Version Number field describes the version of the card capability container. The field is of length one byte; the high order nibble of the byte describes the major version number, and the low order nibble of the byte describes the minor version number.

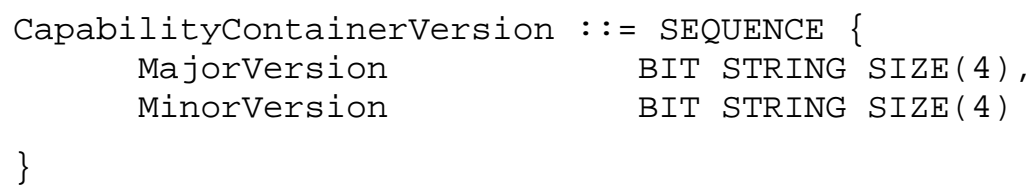

For instance, for this version of the CCC, the high order nibble would contain the number 2, and the low order nibble would contain the number 1, to correspond to version 2.1.

\subsubsection{Capability Grammar Version Number}

The Capability Grammar Version Number field describes the version of the Card Capability Container grammar. The field is of length one byte; the high order nibble of the byte describes the major version number, and the low order nibble of the byte describes the minor version number.

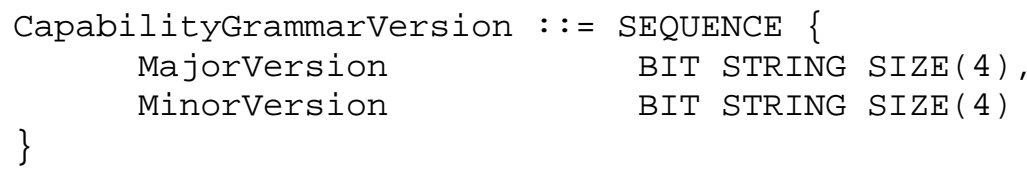

For instance, for this version of the Card Capability Container grammar, the high order nibble would contain the number 2 , and the low order nibble would contain the number 1 , to correspond to version 2.1.

\subsubsection{Applications CardURL Structure}

The Card Capabilities Container may contain multiple instances of ApplicationsCardURL structures, each denoted by the tag value " $0 x F 3$ ". They can be assembled into a list of the applications, including FIDs and paths, Key Identifiers and Access Control Methods, which are supported by the card (see Section 7.1).

The structure of the ApplicationsCardURL is denoted $\{$ T-L- $\{$ T1-L1-V1\} ... TTn-Ln-Vn\}\} with a tag field followed by a length field encoding a number. If the number is not zero, then the value field of the constructed data object, called "template" in ISO/IEC 7816, consists of one or more SIMPLE TLV data objects, each one consisting of a tag field, a length field encoding a number and if the number is not zero, a value field.

\subsubsection{PKCS\#15}

The PKCS\#15 field, if non-zero, indicates that the smart card conforms to PKCS\#15. If the field is nonzero, shall indicate the version of PKCS\#15.

\subsubsection{Registered Daa Model Number}

The Registered Data Model Number indicates the registered Data Model in use by the smart card. 


\subsubsection{Access Cntrol Rules Table}

The Access Control Rules Table allows Access Control Rules to be recorded only once in the card. The table definition is either stored directly in the CCC or in the Access Control Applet (ACA) of a VM card in which case the CCC has a reference to the AID of the Access Control Applet.

For additional information on structure format, see Section 6.3.4.

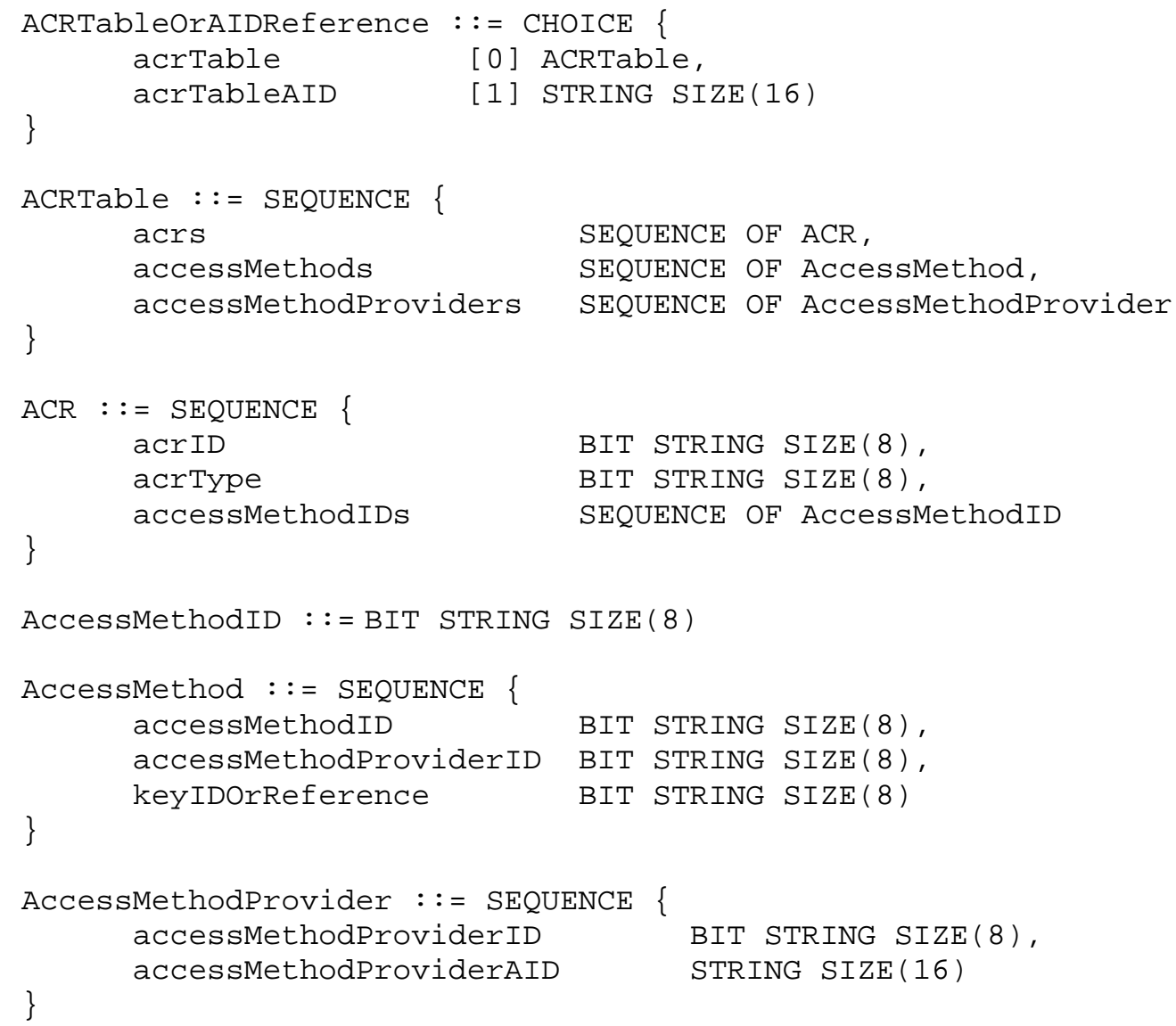

\subsubsection{Card APDUs}

The card capability container optionally may contain a 6-byte Card APDUs field for the purposes of informing the SPS which ISO 7816-4 [ISO4] and 7816-8 [ISO8] APDUs are available on the smart card. Each bit in the string, if set to 1, would indicate the presence of a corresponding APDU. The Card APDUs field is described in more detail in Section 5.2.3.

\subsubsection{Reirection Tag}

In the case an implementer decides that a specific subset of Tags need a particular Security Context and that a specific access control rule should be enforced, it is possible to create a Container for this set of Tags.

The Redirection Tag can be used to indicate to the BSI Provider, Data Model Tags are being "redirected" to the Container. 
The "value" part of the TLV for this redirection Tag can be described as follows:

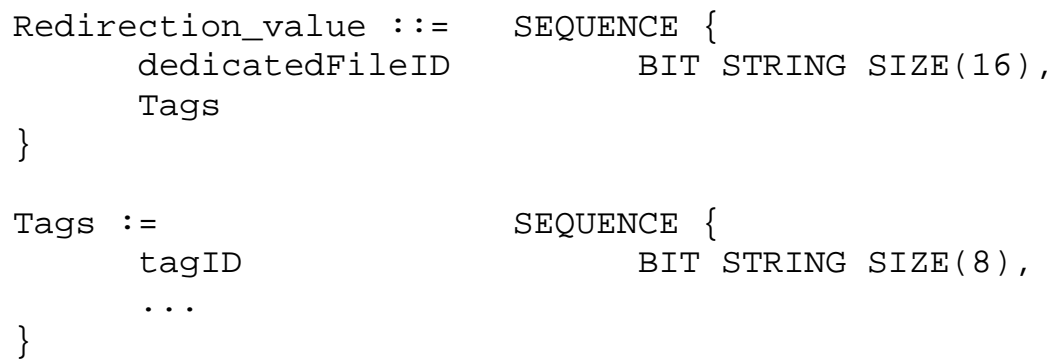

where each "tagID" is a redirected tag.

A DM can have any number of "redirection flags" to handle Tag level exceptions to the nominal DM.

\subsubsection{Capability and Status Tuples}

The CCC shall contain a single Capability Tuple (CT) object, which consists of a collection of two byte tuples defining the capabilities, formats and procedures supported by the smart card. The VCEI defines a default set of APDUs that represent a generic implementation of the ISO 7816 standard. It is only necessary to include CT's to indicate a variance between a given smart card's capabilities and the default set.

The CCC may contain a single Status Tuple (ST) object, consisting of a collection of three byte tuples that define the possible status codes for each function. It is only necessary to include STs that differ from the VCEI's status codes and the status codes defined in ISO 7816-4 [ISO4].

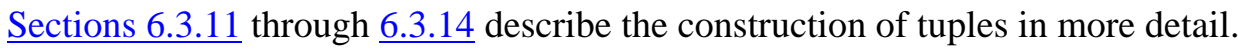

\subsubsection{Capability Tuples}

The CCC shall contain a sequence of two-byte elements called tuples. Each tuple comprises a C-byte and a V-byte as shown in Table 6-2. Each tuple describes one piece of an APDU for a particular command. For example, one tuple may define the value of the CLA byte for a SELECT FILE APDU, while another tuple may define the value of $\mathrm{P} 1$ for the same command.

Table 6-2: Tuple Byte Descriptions

\begin{tabular}{|c|c|c|c|c|c|c|c|}
\hline \multicolumn{7}{|c|}{ C - Code Byte } \\
\hline 7 & 6 & 5 & 4 & 3 & 2 & 1 & 0 \\
\hline $0=$ Const & Parameter & \multicolumn{3}{|l|}{ Function Code } \\
\cline { 1 - 1 } 1= Desc & \multicolumn{1}{|c|}{} \\
\hline
\end{tabular}

\begin{tabular}{|c|c|c|c|c|c|c|c|}
\hline \multicolumn{7}{|c|}{ V - Value/Descriptor Byte } \\
\hline 7 & 6 & 5 & 4 & 3 & 2 & 1 & 0 \\
\hline If $\mathrm{C}$ bit $7=0$ Then $\mathrm{V}$ contains a constant value \\
\hline If $\mathrm{C}$ bit $7=1$ Then $\mathrm{V}$ contains a Descriptor code \\
\hline
\end{tabular}

The C-byte of the tuple is the Code Byte. It identifies the particular command and parameter that is being defined. The V-byte is the Value Byte, which provides either the value to be used for the parameter or a descriptor code that represents the definition of the parameter, that is, what the parameter is in the APDU. This could be, for example, the most-significant byte of the offset for a READ BINARY APDU, or the CHV level for a VERIFY PIN APDU. Whether the V-byte is a constant value or a descriptor code is determined by the $7^{\text {th }}$ bit (most significant bit) of the $\mathrm{C}$-byte. If this bit is 0 , the $\mathrm{V}$-byte contains a value 
while, if it is 1 , the V-byte contains a descriptor code. Bits 6 through 4 of the C-byte identify the parameter and bits 3 through 0 identify the particular command.

The possible values for the codes used in the $\mathrm{C}$ and V-bytes are summarized in Table 6-3.

Table 6-3: Parameter and Function Codes

\begin{tabular}{|c|c|c|c|}
\hline \multicolumn{2}{|c|}{$\begin{array}{l}\text { Parameter } \\
\text { Codes }\end{array}$} & \multicolumn{2}{|r|}{ Function Codes } \\
\hline $0 \times 00$ & DATA & $0 \times 00$ & Reserved, Used for Shift Operation (see Section 6.4.2) \\
\hline $0 \times 01$ & CLA & $0 \times 01$ & Select DF \\
\hline $0 \times 02$ & INS & $0 \times 02$ & Transparent Read (Binary) \\
\hline $0 \times 03$ & P1 & $0 \times 03$ & Update Binary File \\
\hline $0 \times 04$ & P2 & $0 \times 04$ & RFU \\
\hline $0 \times 05$ & P3* & $0 \times 05$ & Manage Security Environment \\
\hline $0 \times 06$ & Prefix & $0 \times 06$ & Get Challenge \\
\hline $0 \times 07$ & Suffix & $0 \times 07$ & Get Response \\
\hline & & $0 \times 08$ & Verify (CHV) \\
\hline & & $0 \times 09$ & Internal Authenticate \\
\hline & & $0 \times 0 \mathrm{~A}$ & External Authenticate \\
\hline & & $0 \times 0 \mathrm{~B}$ & Perform Security Operation \\
\hline & & $0 \times 0 \mathrm{C}$ & Select File \\
\hline & & $0 \times 0 D$ & Select EF (under current DF) \\
\hline & & $0 \times 0 E$ & Select MF (root) \\
\hline & & $0 \times 0 F$ & RFU \\
\hline
\end{tabular}

* Note $:$ P3 is a Length (Lc or Le)

\subsubsection{Prefix and Suffix Codes}

Parameter codes 06 (hexadecimal) and 07 represent prefix and suffix commands respectively. These are commands (function codes) that must execute before or after the specified function code. For example, on some smart cards, a GET RESPONSE must succeed a cryptographic function, or a VERIFY must precede a READ BINARY with secure messaging.

\subsubsection{Descriptor Codes}

The descriptor codes are used to add processing information for data values or parameters. Parameters can be described by at most one descriptor code, whereas data values can be described by multiple, successive descriptor codes. Table 6-10 presents a summary of all descriptor codes.

\subsubsection{Status Tuples}

The purpose of the Status Tuples is to map a smart card's non-standard status response SW1 \& SW2 into a common set of status conditions for a given function. It is not mandatory to list any status conditions that conform to ISO-7816. Status Tuples shall consist of three bytes, labeled S, SW1 and SW2, which describe the possible status conditions for each function. Multiple sets of SW1 and SW2 may translate 
into a single Status Condition. Tables 6-4 through $\underline{6-6}$ describe the status tuple construction and status condition codes.

Table 6-4: Status Tuples

\begin{tabular}{|l|l|l|l|l|l|l|l|}
\hline \multicolumn{10}{|c|}{ S } \\
\hline 7 & 6 & 5 & 4 & 3 & 2 & 1 & 0 \\
\hline \multicolumn{1}{|l|}{ Status Condition } & \multicolumn{6}{|c|}{ Function Code } \\
\hline
\end{tabular}

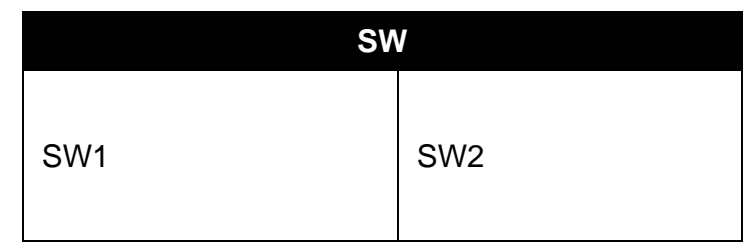

Table 6-5: Standard Status Code Responses

\begin{tabular}{|l|l|}
\hline \multicolumn{2}{|c|}{ Status Conditions } \\
\hline 0x00 & Successful Completion \\
\hline 0x01 & Successful Completion - Warning 1 \\
\hline 0x02 & Successful Completion - Warning 2 \\
\hline 0x03 & Reserved \\
\hline 0x04 & Reserved \\
\hline 0x05 & Reserved \\
\hline 0x06 & Reserved \\
\hline 0x07 & Reserved \\
\hline 0x08 & Access Condition not Satisfied \\
\hline 0x09 & Function not Allowed \\
\hline 0x0A & Inconsistent Parameter \\
\hline 0x0B & Data Error \\
\hline 0x0C & Wrong Length \\
\hline 0x0D & Function not compatible with file structure \\
\hline 0x0E & File/Record not Found \\
\hline 0x0F & Function Not Supported \\
\hline
\end{tabular}

\subsubsection{Next CCC Description}

This field, if included, is used to point to another CCC container. The values in this next CCC container will override values in the current CCC or define new values and fields. The Next CCC field contains an ApplicationsCardURL structure, with minimum length of 20 bytes.

\subsection{Formal Grammar Definition}

Using a modified Backus-Naur notation, a definition for the Card Capability Grammar is presented as follows:

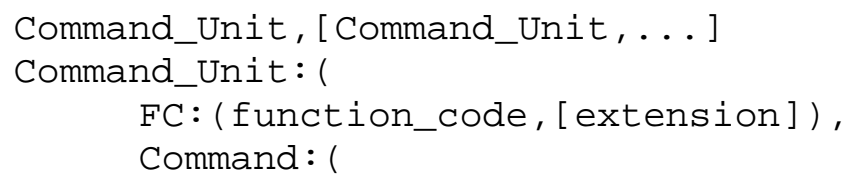




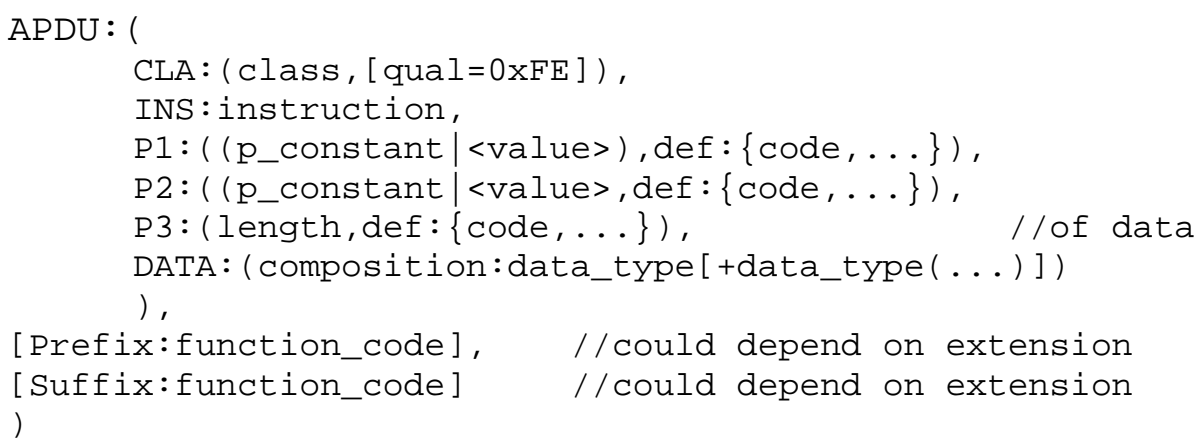

\subsubsection{Grammar Rules}

A description of the symbols follows:

\begin{tabular}{|l|l|}
\hline \multicolumn{1}{|c|}{ Symbol } & \multicolumn{1}{c|}{ Meaning } \\
\hline$:$ & is composed of \\
\hline[] & optional element \\
\hline() & includes or included in \\
\hline, & separates elements \\
\hline$\ldots$ & element repeats unspecified number of times \\
\hline\{\} & choose one from list \\
\hline$<>$ & element value must be given at execution time \\
\hline $\mid$ & or, indicates choice of possibilities for element value \\
\hline+ & element is combined with preceding element \\
\hline$/ /$ & remainder of line contains comments \\
\hline
\end{tabular}

In general, the word immediately preceding a colon is the name of the element, while the word to the right of the colon is the name of an element value that may be expected. A description of the element values is given as follows:

\begin{tabular}{|l|l|}
\multicolumn{1}{|c|}{ Element } & \multicolumn{1}{c|}{ Meaning } \\
\hline Function_code & $\begin{array}{l}\text { value from function code table, always required when other elements } \\
\text { are present }\end{array}$ \\
\hline Class & value for the APDU CLA byte, when entered this is a constant \\
\hline Instruction & value for APDU INS byte, when entered this is a constant \\
\hline Extension & (see discussion about extended function code) \\
\hline P_constant & value for the APDU P1 or P2 byte, when entered this is a constant \\
\hline Code & code for parameter definition, the code must be in the descriptor table \\
\hline Length & length of data element, when entered this is a constant \\
\hline Data_type & $\begin{array}{l}\text { code for the composition of the APDU Data field, must be in the } \\
\text { descriptor table }\end{array}$ \\
\hline Qual & $\begin{array}{l}\text { Qualifier for CLA; only possible value is 0xFE to indicate command is } \\
\text { not available }\end{array}$ \\
\hline
\end{tabular}


Note that all elements except function_code are essentially optional in a command_unit. The square brackets [ ] are used to emphasize that the enclosed optional elements can only be present if the preceding element is present.

The rules for building and APDU definition according to the formal grammar are as follows:

- The sequence of tuples is organized in groups called command units; all tuples pertaining to a single command unit must be presented in contiguous sequence.

- The sequence of tuples is important and must be presented in the order defined by the formal grammar.

- Each command unit consists of a required function code and optional APDU elements.

- When present, the CLA element may have a constant value (and/or one qualifier code equal to 0xFE, which indicates the command is not available on the smart card).

- When present, the INS element must have a constant value.

- When present, the P1 element may optionally have a constant value and/or one/multiple definition code.

- When present, the P2 element may optionally have a constant value and/or one/multiple definition code.

- When present, the P3 element may have a constant value; P3 always refers to the length of the DATA element in the Command APDU or the length of the expected DATA element in the Response APDU (respectively Lc or Le).

- The DATA element may have multiple data type codes; when combined the data type codes define the composition of the value to be placed in the APDU data field.

As an example of using the Card Capability Grammar, consider the following GSC-IS-default APDU for a Select Dedicated File command along with the same command for the Schlumberger [CCPG] card:

Table 6-6: Default vs. Schlumberger DF APDU

\begin{tabular}{|l|l|l|l|l|l|c|}
\hline \multicolumn{7}{|c|}{ Select Dedicated File (DF) } \\
\hline \multicolumn{1}{|c|}{ Card Type } & CLA & INS & P1 & P2 & P3 & Data \\
\hline GSC-IS Default & 00 & A4 & 01 & 00 & L (02) & File ID (2 bytes) \\
\hline Schlumberger Cryptoflex & C0 & A4 & 00 & 00 & L (02) & File ID (2 bytes) \\
\hline
\end{tabular}

The formal grammar definition of the Cryptoflex command is as follows:

$$
\text { FC:01, CLA:C0, INS:A4, P1:00, P2:00, P3:(02,def:15), DATA:21 }
$$

which translates into the following tuple sequence:

$$
\text { 11C0 21A4 } 310041005102 \text { D115 } 8121
$$


The method for creating the tuple sequence is shown in the Table 6-7, where the C-Byte and V-Byte are built from the parameter, function, and descriptor codes given in the Table 6-3 and Table $\underline{6-10}$.

Table 6-7: Tuple Creation Sequence

\begin{tabular}{|c|c|c|c|c|c|c|c|c|}
\hline$\#$ & \multicolumn{3}{|c|}{ C-Byte } & V-Byte & \multicolumn{3}{|c|}{ Description } & Tuple \\
\hline & $\mathbf{s}$ & $\mathbf{P}$ & FC & & Function, Parm & $\mathbf{V} / \mathbf{D}$ & Value/Descriptor & \\
\hline 1 & 0 & 1 & 1 & $\mathrm{CO}$ & Select File, CLA & $\mathrm{V}$ & "CO" & $11 \mathrm{CO}$ \\
\hline 2 & 0 & 2 & 1 & A4 & Select File, INS & $\mathrm{V}$ & "A4" & $21 \mathrm{~A} 4$ \\
\hline 3 & 0 & 3 & 1 & 00 & Select File, P1 & V & "00" & 3100 \\
\hline 4 & 0 & 4 & 1 & 00 & Select File, P2 & $\mathrm{V}$ & "00" & 4100 \\
\hline 5 & 0 & 5 & 1 & 02 & Select File, P3 & $\mathrm{V}$ & "02" & 5102 \\
\hline 6 & 1 & 5 & 1 & 15 & Select File, P3 & $\mathrm{D}$ & Length & D115 \\
\hline 7 & 1 & 0 & 1 & 21 & Select File, Data & D & 2 byte FID & 8121 \\
\hline
\end{tabular}

Table 6-7 shows the complete tuple sequence to define the SELECT DF command for the Cryptoflex[CCPG] card according to the CC Grammar; however, the only differences in the APDU between the GSC-IS Default and the Cryptoflex card are the CLA byte and the P1 parameter. Therefore, only two tuples are necessary since the rest of the APDU is defined by the GSC-IS VCEI. The tuples required to define this SELECT DF command for the Cryptoflex card would be:

Table 6-8: Derived Select DF Tuple

\begin{tabular}{|c|c|c|c|c|c|c|c|c|}
\hline$\#$ & \multicolumn{3}{|c|}{ C-Byte } & V-Byte & \multicolumn{3}{|c|}{ Description } & Tuple \\
\hline & $\mathbf{s}$ & $\mathbf{P}$ & FC & & Function, Parameter & V/D & Value/Descriptor & \\
\hline 1 & 0 & 1 & 1 & $\mathrm{CO}$ & Select File, CLA & V & "CO" & $11 \mathrm{CO}$ \\
\hline 2 & 0 & 3 & 1 & 00 & Select File, P1 & $\mathrm{V}$ & "00" & 3100 \\
\hline
\end{tabular}

\subsubsection{Extended Function Codes}

The construction of the Code Byte allows only four bits for the designation of the function code; however, it may, at times, be necessary to use more than the allocated commands. For example, prefix or suffix commands that are card specific may be required to fulfill the processing for the GSC-IS command on a particular smart card.

The reserve function code " $0 \mathrm{x} 00$ " is used to define a shift tuple. This tuple is used in the sequence of tuples to place all following function codes in a shift state defined by the high-order four bits of the shift key. The function codes are logically or'ed with the current shift tuple to create an extended function code. Placing another shift tuple in the tuple stream places function codes in an un-shift or other shift state. A diagram illustrating the mechanics is given in Figure 6-3. 


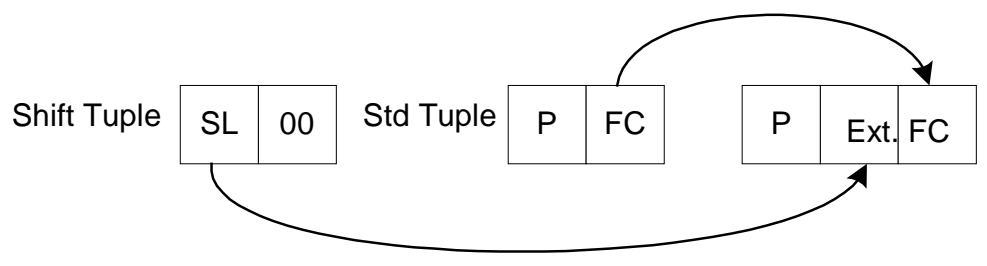

SL: Shift Level; FC: Function Code; P:Parameter; Ext.: Extension

Figure 6-3: Shift Tuple Sequence (SL: shift level)

As an example of using the shift tuple, consider the following sequence of tuples in Table 6-9:

Table 6-9: Example of Extended Function Code

\begin{tabular}{|c|c|c|c|c|c|c|c|c|}
\hline$\#$ & \multicolumn{3}{|c|}{ C-Byte } & V-Byte & \multicolumn{3}{|c|}{ Description } & Tuple \\
\hline & $\mathbf{s}$ & $\mathbf{P}$ & FC & & Function, Parm & V/D & Value/Descriptor & \\
\hline 1 & 0 & 7 & 8 & $1 \mathrm{C}$ & Verify, Suffix & V & $" 1 C "$ & 7817 \\
\hline 2 & 0 & 1 & 0 & 00 & Shift up 1 & V & "00" & 1000 \\
\hline 3 & 0 & 1 & 7 & 00 & Get Response, CLA & $\mathrm{V}$ & "00" & 1700 \\
\hline 4 & 0 & 2 & 7 & $\mathrm{CO}$ & Get Response, INS & V & "CO" & $27 \mathrm{CO}$ \\
\hline 5 & 0 & 5 & 7 & 12 & Get Response, P3 & V & "12" & 5712 \\
\hline 6 & 1 & 5 & 7 & 15 & Get Response, P3 & $\mathrm{D}$ & 15 & D715 \\
\hline 7 & 1 & 0 & 7 & FD & Get Response, Data & D & FD & 87FD \\
\hline 8 & 1 & 0 & 7 & 38 & Get Response, Data & $\mathrm{D}$ & 38 & 8738 \\
\hline 9 & 1 & 0 & 7 & $2 \mathrm{~F}$ & Get Response, Data & D & $2 \mathrm{~F}$ & $872 \mathrm{~F}$ \\
\hline
\end{tabular}

The first two tuples have function code $08 \mathrm{~h}$ indicating a VERIFY command, and give the value for the Data and Suffix parameters. In this case the suffix is a GET RESPONSE with an extended function code. The third tuple is used to set the current shift state. The function codes in the following tuples are logically or'ed with the shift tuple key, which is the C-byte of the shift tuple ("10" in the previous table) to create the extended function code $17 \mathrm{~h}$ (result of $10 \mathrm{~h}$ logically or'ed with $07 \mathrm{~h}$ ). This extended function code is then used to identify a new command that completely specifies a GET RESPONSE using the constant value " 12 " for P3. In this way a card and command-specific length can be specified for the GET RESPONSE. 
Table 6-10: Descriptor Codes

\begin{tabular}{|c|c|c|}
\hline Code & Meaning & Comments \\
\hline $0 \times 00-0 \times 0 F$ & Execute Function Code & \\
\hline $0 \times 11$ & Challenge & $\begin{array}{l}\text { Card Random Number: a designated number of random byte values generated } \\
\text { by the smart card. }\end{array}$ \\
\hline $0 \times 12$ & Algorithm Identifier & \\
\hline $0 \times 13$ & RFU & \\
\hline $0 \times 14$ & RFU & \\
\hline $0 \times 15$ & Length & \\
\hline $0 \times 16$ & MSB of Offset & The most significant byte of the file offset in bytes. \\
\hline $0 \times 17$ & LSB of Offset & The least significant byte of the file offset in bytes. \\
\hline $0 \times 18$ & Key Level & $\begin{array}{l}\text { If the designated key is at the current level (local) insert the byte 0x80; } \\
\text { otherwise, if the key is at the root level (global) insert the byte 0x00. }\end{array}$ \\
\hline $0 \times 19$ & Key Identifier & Key number \\
\hline $0 \times 1 A$ & CHV Level & \\
\hline $0 \times 1 B$ & CHV Identifier & CHV number on smart card \\
\hline $0 \times 1 C$ & AID & Application Identifier \\
\hline $0 \times 1 \mathrm{D}$ & EF & The File ID of an Elementary File \\
\hline $0 \times 1 E$ & SID & The Security Identifier value used by Microsoft Windows. ${ }^{T M}$ \\
\hline $0 \times 1 F$ & Parameter is not used & \\
\hline $0 \times 20$ & RFU & Reserved for future use \\
\hline $0 \times 21$ & 2 Byte FID & The 2-byte File Identifier of the file being accessed. \\
\hline $0 \times 22$ & Short FID & The 5 least significant bits of the 2-byte File Identifier of the file being accessed. \\
\hline $0 \times 23$ & File Name & \\
\hline $0 \times 24$ & AES-ECB & AES algorithm, mode ECB \\
\hline $0 \times 25$ & AES-CBC & AES algorithm, mode $C B C$ \\
\hline $0 \times 26$ & DES & DES algorithm \\
\hline $0 \times 27$ & DES3_16 & Triple DES algorithm \\
\hline $0 \times 28$ & Plain Text & un-encrypted ANSI text \\
\hline $0 \times 29$ & RFU & \\
\hline $0 \times 2 A$ & Pad Data with 0s & The Data is padded at the end with low values to length of $P 3$ \\
\hline $0 \times 2 B$ & PIN & PIN value \\
\hline $0 \times 2 \mathrm{C}$ & 2-byte Key File Identifier & The 2-byte File Identifier of the file of the key being referenced. \\
\hline $0 \times 2 D$ & PIN Type & Pin Type \\
\hline $0 \times 2 E$ & RFU & \\
\hline $0 \times 2 F$ & 8 Byte Random Number & \\
\hline $0 \times 30$ & Length +6 & Length of data plus 6 bytes \\
\hline $0 \times 31$ & Length +3 & Length of data plus 3 bytes \\
\hline $0 \times 32$ & Max Buffer Size & Maximum buffer size in preceding data bytes \\
\hline $0 \times 33$ & n (modulus length) & Used in the RSA algorithm \\
\hline $0 \times 34$ & Message & Plain text message to be encrypted \\
\hline $0 \times 35$ & 4 Byte Word & Length or offset is given in words (one word $=4$ bytes) \\
\hline $0 \times 36$ & Pad Data with FF & Data padded at end with high values \\
\hline
\end{tabular}




\begin{tabular}{|c|c|c|}
\hline Code & Meaning & Comments \\
\hline $0 \times 37$ & $\begin{array}{l}\text { Length = SW2 (with SW1 } \\
=61)\end{array}$ & Length = low nibble of SW1-SW2 (61nn) from last response \\
\hline $0 \times 38$ & RFU & \\
\hline $0 \times 39$ & $\mathrm{RSA}=512$ & RSA 512 bit algorithm using Chinese Reminder Theorem \\
\hline $0 \times 3 A$ & $\mathrm{RSA}=768$ & RSA 768 bit algorithm using Chinese Reminder Theorem \\
\hline $0 \times 3 B$ & $\mathrm{RSA}=1024$ & RSA 1024 bit algorithm using Chinese Reminder Theorem \\
\hline $0 \times 3 C$ & Pad $=$ FF at beginning & Padding (FF) put at the beginning for the length of key to be 128 bytes \\
\hline $0 \times 3 D$ & ANSI X9.31 Padding & \\
\hline $0 \times 3 E$ & Pad $=00(8)$ & $\begin{array}{l}\text { Data padded at the end with low values to the 8-byte boundary (ISO } 9797.2 \\
\text { paragraph } 5.1 \text { method 1). }\end{array}$ \\
\hline $0 \times 3 \mathrm{~F}$ & $\mathrm{Pad}=\mathrm{FF}(128)$ & Data padded at end with high values to total length of 128 bytes (PKCS\#1) \\
\hline $0 \times 40$ & Pad = FF(Front) & \\
\hline $0 \times 41$ & MD5 Header & \\
\hline $0 \times 42$ & LSN Key Encoding & $\begin{array}{l}\text { Concatenate least significant nibbles of key. For example } 8 \text { byte key can be } \\
\text { represented by } 4 \text { bytes. }\end{array}$ \\
\hline $0 \times 43$ & Terminal Random Number & $\begin{array}{l}\text { A designated number of random byte values generated on the terminal by the } \\
\text { BSI. }\end{array}$ \\
\hline $0 \times 44$ & Key Level + Key & Most significant bit is global/local flag \\
\hline $0 \times 45$ & Key File Short ID & $\begin{array}{l}\text { The } 5 \text { least significant bits of the 2-byte File Identifier of the file of the key being } \\
\text { referenced. }\end{array}$ \\
\hline $0 \times 46$ & MSB of Offset in Words & The most significant byte of the file offset in 4 byte words. \\
\hline $0 \times 47$ & LSB of Offset in Words & The least significant byte of the file offset in 4 byte words. \\
\hline $0 \times 48$ & RFU & \\
\hline $0 \times 49$ & Block Length & \\
\hline $0 \times 4 \mathrm{~A}$ & TLV Format & \\
\hline $0 \times 4 \mathrm{~B}$ & Operation Mode & Cryptographic operation modes \\
\hline $0 \times 4 C$ & LOUD & $\begin{array}{l}\text { Length of useful data: the number of bytes in the data transmitted, without } \\
\text { counting any padding or added bytes. }\end{array}$ \\
\hline $0 \times 4 \mathrm{D}$ & RFU & \\
\hline $0 \times 4 E$ & 8 byte Cryptogram & $\begin{array}{l}\text { The cryptogram is generated by encryption of an 8-byte random number with a } \\
\text { designated key, with DES encryption for an 8-byte key and DES3 encryption for } \\
\text { a 16-byte key. }\end{array}$ \\
\hline $0 \times 4 \mathrm{~F}$ & RFU & \\
\hline $0 \times 50$ & Length $+X$ & $\begin{array}{l}\text { The number of bytes to be read or written plus } X \text {, where } X \text { is the smallest value } \\
\text { such that Length }+3+X \text { is evenly divisible by } 8 .\end{array}$ \\
\hline $0 \times 51$ & Pad with X 0xFF Bytes & $\begin{array}{l}\text { Pad data to be read or written with } X 0 \times F F \text { bytes where } X \text { is defined in } \\
\text { descriptor code } 0 \times 50 .\end{array}$ \\
\hline $0 \times 52$ & $\begin{array}{l}\text { Select child DF of current } \\
\text { DF }\end{array}$ & $\begin{array}{l}\text { Descriptor code used to describe variation of the ISO Select file command for } \\
\text { P1 (Function code "OxOC") See section 5.1.1.4 }\end{array}$ \\
\hline $0 \times 53$ & Length + 8 & The number of bytes of data to be read or written plus 8. \\
\hline $0 \times 54$ & Select EF of current DF & $\begin{array}{l}\text { Descriptor code used to describe variation of the ISO Select file command for } \\
\text { P1 (Function code "OxOC") See section 5.1.1.4 }\end{array}$ \\
\hline $0 \times 55$ & $\begin{array}{l}\text { Select parent DF of current } \\
\text { DF }\end{array}$ & $\begin{array}{l}\text { Descriptor code used to describe variation of the ISO Select file command for } \\
\text { P1 (Function code "OxOC") See section 5.1.1.4 }\end{array}$ \\
\hline $0 \times 56$ & $\begin{array}{l}\text { TLV Command Data for } \\
\text { Update Binary }\end{array}$ & $\begin{array}{l}\text { Insert the tag byte } 0 \times 81, \text { the length byte representing the number of data bytes } \\
\text { to be written to the smart card, and the data bytes to be written. }\end{array}$ \\
\hline
\end{tabular}




\begin{tabular}{|c|c|c|}
\hline Code & Meaning & Comments \\
\hline $0 \times 57$ & $\begin{array}{l}\text { TLV Response for Update } \\
\text { Binary }\end{array}$ & $\begin{array}{l}\text { Interpret as the tag byte 0x99, the length byte 0x02, and two data bytes } \\
\text { representing ISO 7816-4 status bytes SW1 and SW2. }\end{array}$ \\
\hline $0 \times 58$ & $\begin{array}{l}\text { TLV Command Data for } \\
\text { Read Binary }\end{array}$ & $\begin{array}{l}\text { Insert the tag byte } 0 \times 97 \text {, the length byte } 0 \times 01 \text {, and a byte representing the } \\
\text { number of bytes to be read from the smart card. }\end{array}$ \\
\hline $0 \times 59$ & $\begin{array}{l}\text { TLV Response Data for } \\
\text { Read Binary }\end{array}$ & $\begin{array}{l}\text { Interpret as the tag byte } 0 \times 81 \text {, the length byte representing the number of data } \\
\text { byte read from the smart card, and the data bytes read. }\end{array}$ \\
\hline $0 \times 5 A$ & DES3_16-ECB & Triple DES algorithm, 16 bytes key, ECB mode, \\
\hline $0 \times 5 B$ & DES3_16-CBC & Triple DES algorithm, 16 bytes key, CBC mode, \\
\hline $0 \times 5 C$ & DES-ECB & DES algorithm, mode ECB \\
\hline $0 \times 5 D$ & DES-CBC & $D E S$ algorithm, mode $C B C$ \\
\hline $0 \times 5 E$ & RSA $=2048$ & RSA 2048 bit algorithm using Chinese Reminder Theorem \\
\hline $0 \times 5 \mathrm{~F}$ & Key Number $<<1$ & $\begin{array}{l}\text { The number of the designated key is shifted } 1 \text { bit to the left (equal to multiplying } \\
\text { the key number by 2). }\end{array}$ \\
\hline $0 \times 60$ & Key Level Flag & $\begin{array}{l}\text { If the designated key is at the current level (local) insert the byte } 0 \times 80 \text {; } \\
\text { otherwise, if the key is at the root level (global) insert the byte } 0 \times 00 .\end{array}$ \\
\hline $0 \times 61$ & Length + \#Padding & $\begin{array}{l}\text { The length of the data transmitted plus the number of padding bytes required to } \\
\text { fill the designate block size: } 64 \text { bytes for an RSA 512-bit key, } 96 \text { bytes for an } \\
R S A \text { 768-bit key, and } 128 \text { bytes for an RSA 1024-bit key }\end{array}$ \\
\hline $0 \times 62$ & Length of RSA Response & $\begin{array}{l}\text { The response length is the same as the padded length of data sent to the smart } \\
\text { card in an RSA Compute command. }\end{array}$ \\
\hline $0 \times 63$ & RSA Response Data & $\begin{array}{l}\text { Interpret as the return data from an RSA Compute command: a digital signature } \\
\text { computed for a padded hash sent to the smart card, or a decrypted padded } \\
\text { hash for a digital signature sent to the smart card. }\end{array}$ \\
\hline $0 \times 64$ & $\begin{array}{l}\text { Pad Hashed Data } \\
\text { (PKCS\#1) }\end{array}$ & 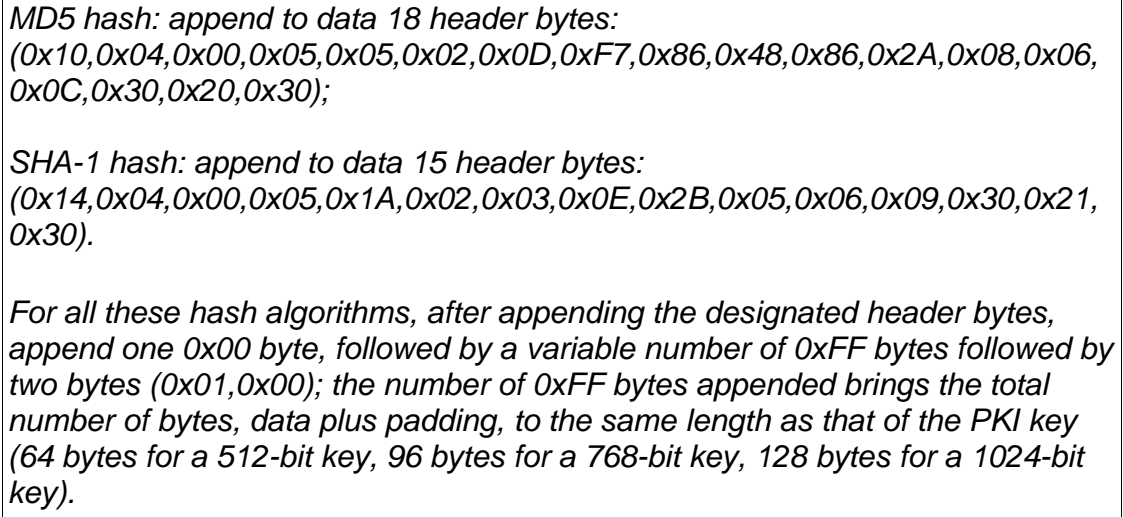 \\
\hline $0 \times 65$ & Swap Data Bytes & $\begin{array}{l}\text { The data bytes (either command data sent to the smart card or response data } \\
\text { received from the smart card) are swapped, so that for } N \text { bytes, the } 1 \text { st } \\
\text { swapped byte is the Nth data byte, the } 2 \text { nd swapped byte is the N-1st and so } \\
\text { forth, until the Nth swapped byte is the } 1 \text { st data byte. }\end{array}$ \\
\hline $0 \times 66$ & TLV Key ID & $\begin{array}{l}\text { Insert the tag byte 0x84, the length byte 0x01, and a byte representing the key } \\
\text { identifier of the key used in the PKI computation. }\end{array}$ \\
\hline $0 \times 67$ & TLV Hash Algorithm ID & $\begin{array}{l}\text { Insert the tag byte } 0 \times 80, \text { the length byte } 0 \times 01, \text { and a byte representing the } \\
\text { algorithm used to hash the data being signed: } 0 \times 32 \text { for MD5 or } 0 \times 12 \text { for SHA-1. }\end{array}$ \\
\hline $0 \times 68$ & $\begin{array}{l}\text { Key Length Padded Hash } \\
\text { Data }\end{array}$ & $\begin{array}{l}\text { The first byte of the data is a value equal to the length of the PKI key being } \\
\text { used, followed by the } 0 \times 00 \text { byte, followed by the swapped padded hashed data } \\
\text { bytes, with padding per descriptor byte 0x65 and swapping per descriptor byte } \\
0 \times 64 .\end{array}$ \\
\hline $0 \times 69$ & Key Length +2 & The value is the length of the PKI key being used plus 2. \\
\hline $0 \times 70-0 \times 99$ & RFU & \\
\hline $0 \times A 0-0 \times D F$ & Implementation Dependent & \\
\hline
\end{tabular}


NIST IR 6887 - 2003 EDITION, GSC-IS VERSION 2.1

\begin{tabular}{|c|l|l|}
\hline Code & \multicolumn{1}{|c|}{ Meaning } & \multicolumn{1}{c|}{ Comments } \\
\hline 0xE0 & Put Data Bytes & Place Data Bytes (En) in data stream output to smart card \\
\hline 0xE1-0xEF & En N Data Bytes & En: Next $n$ bytes are Data Bytes \\
\hline 0xF0-0xFC & Reserved & \\
\hline 0xFD & Interpret Response & Following descriptor bytes are used to interpret response \\
\hline 0xFE & Command not available & Command is not available on smart card \\
\hline 0xFF & User Input Required & Parameter value must be supplied by use/program \\
\hline
\end{tabular}




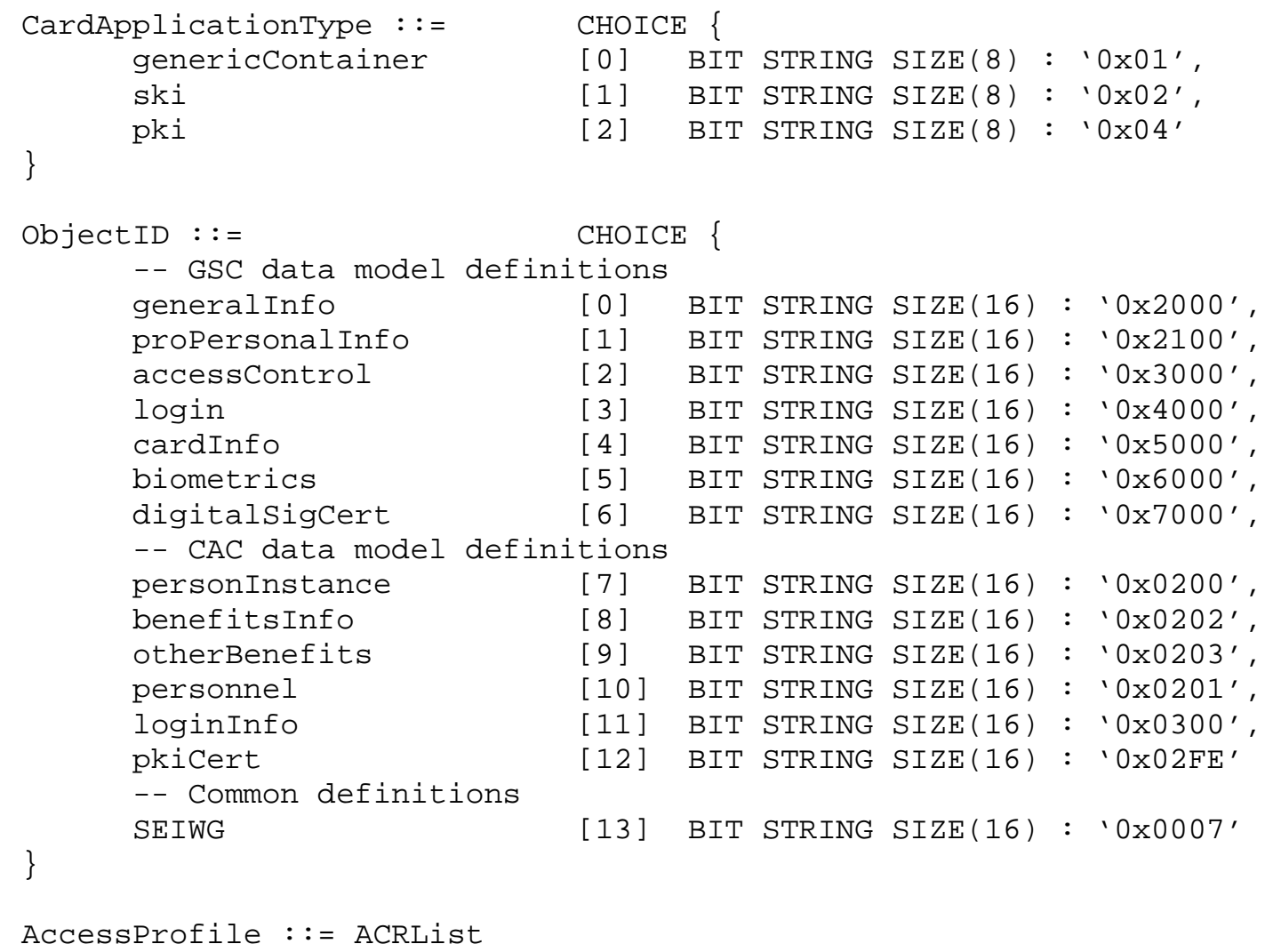




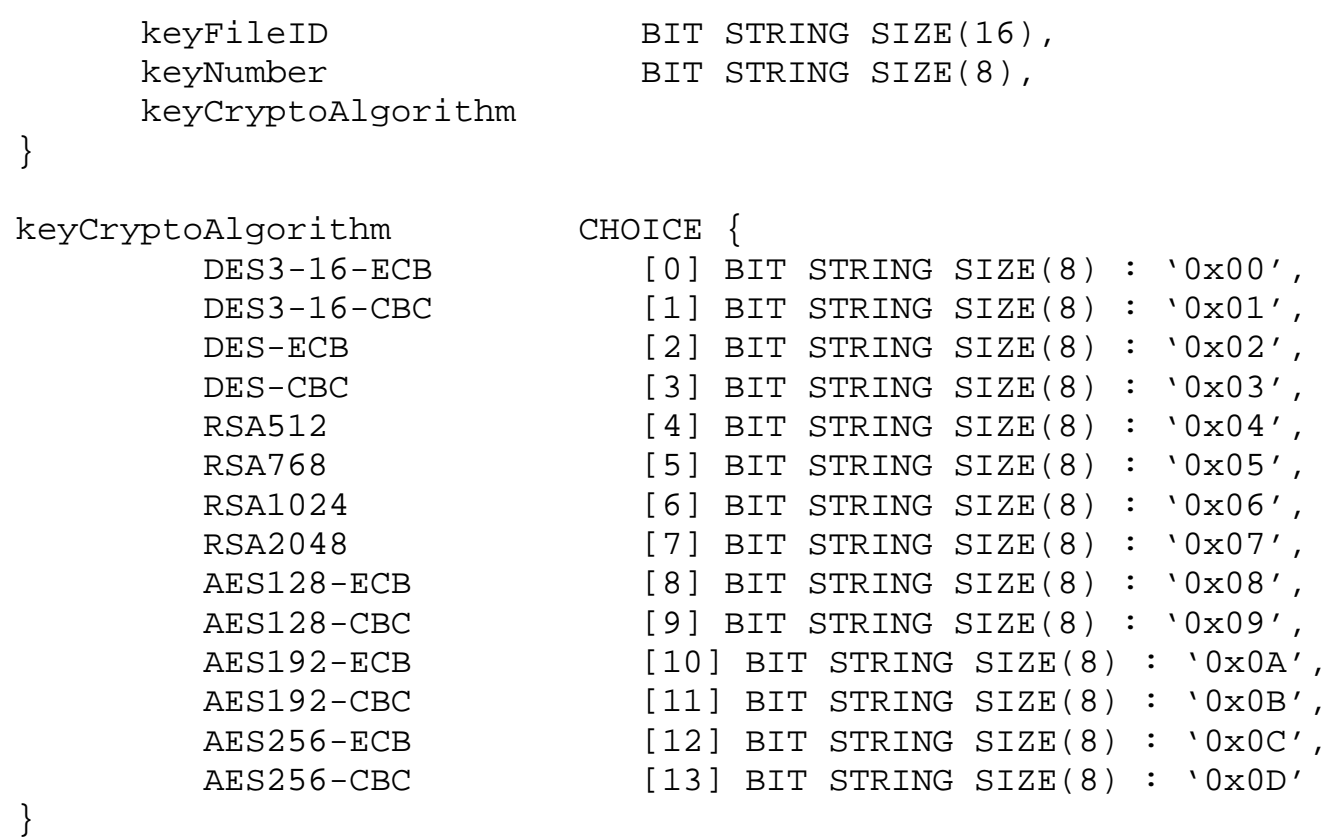

\subsection{Using the Applications CardURL Structure for Container Selection}

The Universal AIDs associated with each data model are published in the appendices of this specification. When a client application attempts to first access a container, it will need to retrieve the ApplicationsCardURL structure that corresponds to that container's Universal AID, and use the information contained therein to access the container. This is done differently for file system and VM smart cards. The RID field contains the registered identifier [ISO5] data model.

\subsection{File System Cards: Selecting Containers}

The ObjectID field in the ApplicationsCardURL structure contains the two-byte File ID of the desired container. In the case of file system cards, the Application ID field will be the two-byte File ID of either the Master File or the Directory file within the Master File.

\subsection{VM Cards: Selecting Containers and Applets}

For VM cards, selecting the container is a two-part process. First, one retrieves the File ID for the desired container from the Object ID field (as with file system cards). Secondly, one retrieves the AID of the applet that manages the container; that applet's AID is found in the ApplicationID field.

\subsection{Using the Applications CardURL Structure for Identifying Access Control Rules}

Identifying the access control rules associated with a specific container is straightforward after the container's associated ApplicationsCardURL structure is retrieved. The value of the AccessProfile field determines whether the following structure is a generic container ACR list (GCACRIist) or a cryptographic service modules ACR list (CryptoACRlist). Note that different access control rules can be associated with reading tags versus reading values. 
NIST IR 6887 - 2003 EDITION, GSC-IS VERSION 2.1

\section{THIS PAGE INTENTIONALLY LEFT BLANK.}




\section{Data Model}

\subsection{Data Model Overview}

Data Models define a set of containers (files) and associated data elements in TLV format. The only mandatory containers are the CCC and Access Control File or SEIWG file. With the exception of the CCC and Access Control File, a GSC-IS conformant card may implement all, some, or none of the other containers associated with a Data Model. However, if the smart card uses any of the data elements defined in Data Model then it must use the container and TLV format specified by that Data Model for that element.

The SEIWG [SEIW] string is defined as the minimum interoperability mechanism for card holder authentication. The SEIWG strings and files are therefore mandatory for both contact and contactless GSC cards.

This specification defines two Data Models. The GSC Data Model was developed for version 1.0 of the GSC-IS (see Appendix C). The GSC Data Model is sometimes referred to as the "J.8" Data Model, since it was first defined in Section J.8 of the Smart Access Common ID Card contract. The second Data Model was developed for the DoD Common Access Card (CAC) and is referred to as the CAC Data Model (see Appendix D).

Applications can discover which Data Model a given card supports by examining the Registered Data Model field of the card's Card Capabilities Container (see Chapter 6). The Registered Data Model field shall contain a 0x01 if the card is using the GSC-IS Data Model defined in Appendix C, or a 0x02 if the card conforms to the CAC Data Model in Appendix D. Error Detection Codes (EDC) are only mandated for the GSC-IS Data Model.

\subsection{Internal Tag-Length-Value Format}

All container data elements are stored in SIMPLE-TLV format. Each SIMPLE-TLV data object shall consist of a tag field, a length field and an optional value field. For VM cards implementing the VM card edge interface, the SIMPLE-TLV format is split into a T-Buffer and V-Buffer. (See description in Section 8.4)

The tag field $\mathrm{T}$ shall consist of a single byte encoding only a number from 1 to 254 . No class or construction types are coded. The values "0x00" and "0xFF" are invalid for tag fields. The tag value $0 x F E$ is reserved for the mandatory EDC data object in each container.

The scope of tag values is at the container level, so the same tag value could appear in different containers and have different meanings. Unique tag values are used across all containers in the current GSC-IS Data Models, although this is not a mandatory requirement.

The length field shall consist of 1 or 3 consecutive bytes. If the leading byte of the length field is in the range from ' 00 ' to ' $\mathrm{FE}$ ', then the length field shall consist of a single byte encoding an integer $\mathrm{L}$ valued from 0 to 254. If the leading byte is equal to 'FF', then the length field continues on the two subsequent bytes in least significant byte (LSB) - most significant byte (MSB) order, which encode an integer L with a value from 0 to 65,535 .

If $\mathrm{L}$ is not zero, then the value field $\mathrm{V}$ shall consist of $\mathrm{L}$ consecutive bytes. If $\mathrm{L}$ is zero or if a tag is omitted from its file/buffer, then the data object must be empty; there is no value field for that tag. 


\subsection{Structure and Length Values for Cards Requiring the File System Card Edge}

The file system card edge requires containers to be implemented as a single file, i.e., one file comprises the container. The first TLV record of the container may optionally contain the length of the occupied space in the container as follows:

Container Byte 0: $\quad$ Tag $=0 x E E$

Container Byte 1: $\quad$ Length $=0 \mathrm{x} 02$

Container Byte 2: $\quad$ Least significant byte of length of occupied space

Container Byte 3: $\quad$ Most significant byte of length of occupied space

Container Byte 4: $\quad$ Next tag value

\subsection{Structure and Length Values for Cards Requiring the Virtual Machine Card Edge}

The virtual machine card edge is designed to interact with containers that are split into two buffers: the TBuffer, for storing the tag and associated tag lengths, and the V-Buffer, for storing the values. The first two bytes of each buffer contain the length of the occupied space in the buffer in LSB-MSB format.

\subsubsection{T-Buffer}

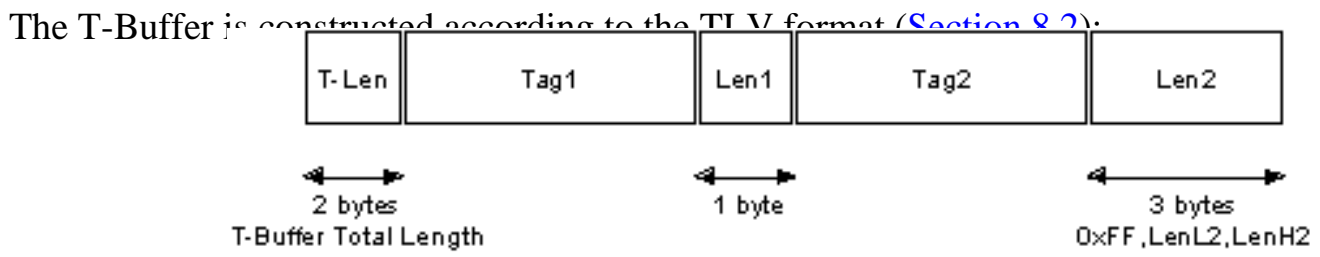

Figure 8-1: T-Buffer Format

\subsubsection{V-Buffer}

The V-Buffer is constructed as follows according to the TLV format:

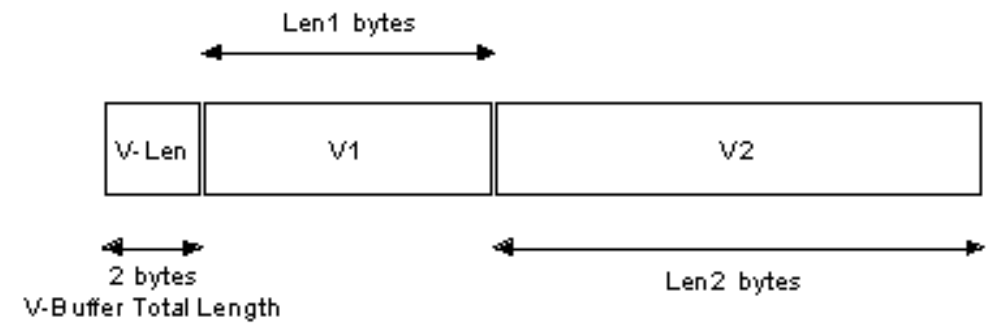

Figure 8-2: V-Buffer Format 


\section{Appendix A-Normative References}

[DES] National Institute of Standards and Technology, "DES Modes of Operation”, Federal Information Processing Standards Publication 81, December 1980, http://csrc.nist.gov/publications/fips

[FIPS1] National Institute of Standards and Technology, Federal Information Processing Standard (FIPS) 140-2: Security Requirements for Cryptographic Modules, Decemeber 3, 2002

[GLOB] Global Platform Specification v2.1, http://www.globalplatform.org.

[ISO3] ISO/IEC 7816-3 1995(E): Electronic Signals and Transmission Protocols, http://www.iso.ch.

[ISO4] ISO/IEC 7816-4 1995(E): Interindustry Commands for Interchange

[ISO5] ISO/IEC 7816-5 1994-1996 (Amendment 1): Numbering system and registration procedure for application identifiers.

[ISO8] ISO/IEC 7816-8 1995(E): Interindustry Commands for a Cryptographic Toolbox

[ISO9] International Organization for Standardization, "Information Processing Systems -- Data Communication High-Level Data Link Control Procedure--Frame Structure", IS 3309, October 1984, 3rd Edition.

[1444] ISO/IEC 14443, Contactless integrated circuit(s) cards - Proximity cards - Parts 1 - 4 
NIST IR 6887 - 2003 EDITION, GSC-IS VERSION 2.1

\section{THIS PAGE INTENTIONALLY LEFT BLANK.}




\section{Appendix B-Informative References}

[OCF] The OpenCard Framework, http://www.opencard.org.

[JAVA] Java Card 2.1.1 Platform Documentation, http://java.sun.com/products/javacard/javacard21.html

[CCCG] GSC-IS CCC Grammar Tutorial, Jackson, Harry, 2001, http://smartcard.nist.gov/cccgrammartutorial.pdf

[PCSC] Personal Computer/Smart Card Workgroup Specifications, http://www.pcscworkgroup.com.

[CCPG] Cryptoflex Cards Programmer's Guide, www.cryptoflex.com

[SEIW] Physical Access Interoperability Working Group (PAIWG) Technical Implementation Guidance, Final Draft v1.0, Smart Card Enabled Physical Access Control Systems (dated 2 July 2003). 
NIST IR 6887 - 2003 EDITION, GSC-IS VERSION 2.1

\section{THIS PAGE INTENTIONALLY LEFT BLANK.}




\section{Appendix C-GSC Data Model}

The RID for the GSC Data Model is 0xA000000116. The registered Data Model number is 0x01, and the Data Model version number is 0x01.

Note that the Access Control File and the SEIWG[SEIW] field contained therein are mandatory for this data model.

\begin{tabular}{|l|l|l|l|}
\hline \multicolumn{1}{|c|}{ File/Buffer Description } & FID & \multicolumn{1}{c|}{$\begin{array}{c}\text { Maximum } \\
\text { Length (Bytes) }\end{array}$} & Read Access Condition \\
\hline Capability & DB00 & & Always Read \\
\hline General Information & 2000 & 509 & Always Read \\
\hline Protected Personal Information & 2100 & 19 & Verify CHV \\
\hline Access Control & 3000 & 59 & Always Read \\
\hline Login & 4000 & 141 & Verify CHV \\
\hline Card Information & 5000 & 165 & Always Read \\
\hline Biometrics - X.509 Certificate & 6000 & 2013 & Always Read \\
\hline PKI - Digital Signature Certificate & 7000 & 3017 & Verify CHV \\
\hline
\end{tabular}

\begin{tabular}{|l|l|l|l|}
\hline \multicolumn{1}{|c|}{ General Information File / Buffer } & EF 2000 Always Read & Max. Bytes \\
\hline First Name & Tag & \multicolumn{1}{c|}{ Type } & 20 \\
\hline Middle Name & 01 & Variable & 20 \\
\hline Last Name & 02 & Variable & 20 \\
\hline Suffix & 03 & Variable & 4 \\
\hline Government Agency & 04 & Variable & 30 \\
\hline Bureau Name & 05 & Variable & 30 \\
\hline Agency Bureau Code & 06 & Variable & 4 \\
\hline Department Code & 07 & Variable & 4 \\
\hline Position/Title & 08 & Variable & 30 \\
\hline Building Name & 09 & Variable & 30 \\
\hline Office Address 1 & 10 & Variable & 60 \\
\hline Office Address 2 & 11 & Variable & 60 \\
\hline Office City & 12 & Variable & 50 \\
\hline Office State & 13 & Variable & 20 \\
\hline Office ZIP & 14 & Variable & 15 \\
\hline Office Country & 15 & Variable & 4 \\
\hline Office Phone & 16 & Variable & 15 \\
\hline Office Extension & 17 & Variable & 4 \\
\hline Office Fax & 18 & Variable & 15 \\
\hline Office Email & 19 & Variable & 60 \\
\hline Office Room Number & $1 \mathrm{~A}$ & Variable & 6 \\
\hline Non-Government Agency & $1 \mathrm{~B}$ & Variable & 1 \\
\hline & $1 \mathrm{C}$ & Fixed Text & \\
\hline & & & \\
\hline
\end{tabular}




\begin{tabular}{|l|c|l|l|l|}
\hline General Information File / Buffer & \multicolumn{2}{c|}{ EF 2000 Tlways Read } & Max. Bytes \\
\hline \multicolumn{1}{|c|}{ Data Element (TLV) } & Tag & \multicolumn{2}{c|}{ Type } & 6 \\
\hline SSN Designator & 1D & Variable & 1 \\
\hline Error Detection Code & FE & LRC & \\
\hline
\end{tabular}

\begin{tabular}{|l|l|l|l|}
\hline \multicolumn{1}{|c|}{ Protected Personal Information File / Buffer Element (TLV) } & \multicolumn{1}{c|}{ Tag } & \multicolumn{1}{c|}{ Type } & Max. Bytes \\
\hline Social Security Number & 20 & Fixed Text & 9 \\
\hline Date of Birth & 21 & Date (YYYYMMDD) & 8 \\
\hline Gender & 22 & Fixed Text & 1 \\
\hline Error Detection Code & FE & LRC & 1 \\
\hline
\end{tabular}

\begin{tabular}{|l|l|l|l|}
\hline $\begin{array}{l}\text { Access Control File / Buffer (Note: File mandatory for contact EF } 3000 \quad \text { Always Read } \\
\text { cards) }\end{array}$ Data Element (TLV) & \multicolumn{1}{|c|}{ Tag } & \multicolumn{1}{|c|}{ Type } & Max. Bytes \\
\hline $\begin{array}{l}\text { SEIWG Data (Note: Field mandatory for } \\
\text { contact cards) }\end{array}$ & 30 & Fixed & $40^{*}$ \\
\hline PIN & 31 & Fixed Numeric & 10 \\
\hline Domain (Facility / System ID) & 32 & Variable & 8 \\
\hline Error Detection Code & FE & LRC & 1 \\
\hline
\end{tabular}

*The SEIWG data format is defined in [SEIW].

\begin{tabular}{|l|l|l|l|l|}
\hline \multicolumn{2}{|c|}{ Login Information File / Buffer } & EF 4000 & Verify CHV \\
\hline User ID & Tag Element (TLV) & \multicolumn{1}{|c|}{ Type } & Max. Bytes \\
\hline Domain & 40 & Variable & 60 \\
\hline Password & 41 & Variable & 60 \\
\hline Error Detection Code & 42 & Variable & 20 \\
\hline
\end{tabular}

\begin{tabular}{|l|c|l|l|l|}
\hline \multicolumn{2}{|c|}{ Card Information File / Buffer } & EF 5000 & Always Read \\
\hline \multicolumn{1}{|c|}{ Data Element (TLV) } & Tag & \multicolumn{1}{c|}{ Type } & Max. Bytes \\
\hline Issuer ID & 50 & Variable & 32 \\
\hline Issuance Counter & 51 & Variable & 4 \\
\hline Issue Date & 52 & Date (YYYYMMDD) & 8 \\
\hline Expiration Date & 53 & Date (YYYYMMDD) & 8 \\
\hline Card Type & 54 & Variable & 32 \\
\hline Demographic Data Load Date & 55 & Date (YYYYMMDD) & 8 \\
\hline Demographic Data Expiration Date & 56 & Date (YYYYMMDD) & 8 \\
\hline Card Security Code & 57 & Fixed Text & 32 \\
\hline Card ID AID & 58 & Variable & 32 \\
\hline
\end{tabular}




\begin{tabular}{|l|l|l|l|l|}
\hline Card Information File / Buffer & EF 5000 & Always Read \\
\hline \multicolumn{1}{|c|}{ Data Element (TLV) } & Tag & & Type & Max. Bytes \\
\hline Error Detection Code & FE & LRC & & 1 \\
\hline
\end{tabular}

\begin{tabular}{|l|l|l|l|l|}
\hline \multicolumn{2}{|c|}{ Biometrics - X.509 Certificate File / Buffer } & EF6000 & Always Read \\
\hline \multicolumn{1}{|c|}{ Data Element (TLV) } & \multicolumn{1}{c|}{ Tag } & \multicolumn{2}{c|}{ Type } & Max. Bytes \\
\hline Template & 60 & Variable & 512 \\
\hline Certificate & 61 & Variable & 1500 \\
\hline Error Detection Code & FE & LRC & 1 \\
\hline
\end{tabular}

\begin{tabular}{|l|l|l|l|l|}
\hline PKI - Digital Signature Certificates File / Buffer & EF 7000 & Verify CHV \\
\hline \multicolumn{1}{|c|}{ Data Element (TLV) } & Tag & \multicolumn{1}{c|}{ Type } & Max. Bytes \\
\hline Certificate & 70 & Variable & 3000 \\
\hline Issue Date & 71 & Date (YYYYMMDD) & 8 \\
\hline Expiration Date & 72 & Date (YYYYMMDD) & 8 \\
\hline Error Detection Code & FE & LRC & 1 \\
\hline
\end{tabular}


NIST IR 6887 - 2003 EDITION, GSC-IS VERSION 2.1

\section{THIS PAGE INTENTIONALLY LEFT BLANK.}




\section{Appendix D-DoD Common Access Card (CAC) Data Model}

\section{D.1 CAC Data Model Specific}

The RID for the all the files except the CCC in the CAC Data Model is 0xA000000079. The registered Data Model number is 0x02, and the Data Model version number is 0x01. The CCC RID is 0xA000000116.

The CAC containers are stored in SIMPLE-TLV format as per $\underline{\text { Chapter } 8 .}$

\begin{tabular}{|l|l|l|l|}
\hline \multicolumn{1}{|c|}{ File/Buffer Description } & \multicolumn{1}{|c|}{ FID } & \multicolumn{1}{|c|}{$\begin{array}{c}\text { Maximum } \\
\text { Length (Bytes) }\end{array}$} & \multicolumn{1}{|c|}{$\begin{array}{c}\text { Read Access } \\
\text { Condition }\end{array}$} \\
\hline Capability & DB00 & & Always Read \\
\hline Person Instance Container & 0200 & 469 & PIN or External Auth \\
\hline Benefits Information Container & 0202 & 19 & PIN or External Auth \\
\hline Other Benefits Container & 0203 & 59 & PIN or External Auth \\
\hline Personnel Container & 0201 & 141 & PIN or External Auth \\
\hline Login Information Container & 0300 & 133 & PIN or External Auth \\
\hline PKI Certificate Container & $02 \mathrm{FE}$ & 2013 & PIN Always \\
\hline SEIWG & 0007 & 41 & Always Read \\
\hline
\end{tabular}

\begin{tabular}{|c|c|c|c|}
\hline Person Instance File/Buffer & & EF 0200 & Always Read \\
\hline Data Element (TLV) & Tag & Type & Max. Bytes \\
\hline Person First Name & 01 & Variable & 40 \\
\hline Person Middle Name & 02 & Variable & 40 \\
\hline Person Last Name & 03 & Variable & 52 \\
\hline Person Cadency Name & 04 & Variable & 8 \\
\hline Person Identifier & 05 & Fixed Text & 30 \\
\hline Date of Birth & 06 & Date (YYYYMMDD) & 16 \\
\hline Sex Category Code & 07 & Fixed Text & 2 \\
\hline Person Identifier Type Code & 08 & Fixed Text & 2 \\
\hline Blood Type Code & 11 & Fixed Text & 4 \\
\hline DoD EDI Person Identifier & 17 & Fixed Text & 20 \\
\hline Organ Donor & 18 & Fixed Text & 2 \\
\hline Identification Card Issue Date & 62 & Date (YYYYMMDD) & 16 \\
\hline Identification Card Expiration Date & 63 & Date (YYYYMMDD) & 16 \\
\hline $\begin{array}{l}\text { Date Demographic Data was Loaded on } \\
\text { Chip }\end{array}$ & 65 & Date (YYYYMMDD) & 16 \\
\hline Date Demographic Data on Chip Expires & 66 & Date (YYYYMMDD) & 16 \\
\hline Card Instance Identifier & 67 & Fixed Text & 2 \\
\hline
\end{tabular}




\begin{tabular}{|l|l|l|l|l|}
\hline SEIWG File / Buffer (Note: File Mandatory for Contact Cards) & EF 0007 & Always Read \\
\hline \multicolumn{1}{|c|}{ Data Element (TLV) } & Tag & \multicolumn{2}{c|}{ Type } & Max. Bytes \\
\hline SEIWG Data & 30 & Fixed & $40^{*}$ \\
\hline Error Detection Code & FE & LRC & 1 \\
\hline
\end{tabular}

*The SEIWG data format is defined in [SEIW].

\begin{tabular}{|l|l|l|l|l|}
\hline \multicolumn{1}{|c|}{ Benefits Information File / Buffer } & EF 0202 & CHV Verify \\
\hline Data Element (TLV) & Tag & \multicolumn{1}{|c|}{ Type } & Max. Bytes \\
\hline Exchange Code & 12 & Fixed Text & 2 \\
\hline Commissary Code & 13 & Fixed Text & 2 \\
\hline MWR Code & 14 & Fixed Text & 2 \\
\hline $\begin{array}{l}\text { Non-Medical Benefits Association End } \\
\text { Date }\end{array}$ & $1 \mathrm{~B}$ & Date (YYYYMMDD) & 16 \\
\hline Direct Care End Date & $1 \mathrm{C}$ & Date (YYYYMMDD) & 16 \\
\hline $\begin{array}{l}\text { Civilian Health Care Entitlement Type } \\
\text { Code }\end{array}$ & D0 & Fixed Text & 2 \\
\hline Direct Care Benefit Type Code & D1 & Fixed Text & 2 \\
\hline Civilian Health Care End Date & D2 & Fixed Text & 16 \\
\hline
\end{tabular}

\begin{tabular}{|c|c|l|l|l|}
\hline Other Benefits File / Buffer & EF 0203 & Always Read \\
\hline Data Element (TLV) & Tag & \multicolumn{2}{|c|}{ Type } & Max. Bytes \\
\hline Meal Plan Type Code & 1A & Fixed Text & 4 \\
\hline
\end{tabular}

\begin{tabular}{|l|c|l|l|l|}
\hline \multicolumn{2}{|c|}{ Personnel File / Buffer } & EF 0201 & Always Read \\
\hline \multicolumn{1}{|c|}{ Data Element (TLV) } & Tag & \multicolumn{1}{|c|}{ Type } & Max. Bytes \\
\hline DoD Contractor Function Code & 19 & Fixed Text & 2 \\
\hline US Government Agency/Subagency Code & 20 & Fixed Text & 8 \\
\hline Branch of Service Code & 24 & Fixed Text & 2 \\
\hline Pay Grade Code & 25 & Fixed Text & 4 \\
\hline Rank Code & 26 & Fixed Text & 12 \\
\hline Personnel Category Code & 34 & Fixed Text & 2 \\
\hline $\begin{array}{l}\text { Non-US Government Agency/Subagency } \\
\text { Code }\end{array}$ & 35 & Fixed Text & 4 \\
\hline Pay Plan Code & 36 & Fixed Text & 4 \\
\hline Personnel Entitlement Condition Code & D3 & Fixed Text & 4 \\
\hline
\end{tabular}

\begin{tabular}{|l|c|l|c|c|c|}
\hline \multicolumn{2}{|l|}{ Login Information File / Buffer } & EF 0300 & CHV Verify \\
\hline \multicolumn{1}{|c|}{ Data Element (TLV) } & Tag & & Type & Max. Bytes \\
\hline User ID & $0 \times 40$ & Variable & 20 \\
\hline
\end{tabular}


NIST IR 6887 - 2003 EDITION, GSC-IS VERSION 2.1

\begin{tabular}{|l|l|l|l|}
\hline Domain & $0 \times 41$ & Variable & 20 \\
\hline Passwordlnfo & $0 \times 43$ & Fixed Text & 1 \\
\hline ApplicationName & $0 \times 44$ & Variable & 8 \\
\hline Error Detection Code & 0xFE & LRC & 1 \\
\hline
\end{tabular}

\begin{tabular}{|l|c|l|l|l|}
\hline \multicolumn{2}{|c|}{ PKI Certificate File / Buffer } & EF 02FE & CHV Verify \\
\hline Data Element (TLV) & Tag & \multicolumn{1}{c|}{ Type } & \multicolumn{1}{c|}{ Max. Bytes } \\
\hline Certificate & $0 \times 70$ & Variable & 1100 \\
\hline CertInfo & $0 \times 71$ & Fixed Text & 1 \\
\hline MSCUID & $0 x 72$ & Variable & 38 \\
\hline Error Detection Code & 0xFE & LRC & 1 \\
\hline
\end{tabular}


NIST IR 6887 - 2003 EDITION, GSC-IS VERSION 2.1

\section{THIS PAGE INTENTIONALLY LEFT BLANK.}




\section{Appendix E-C Language Binding for BSI Services}

This appendix defines the $\mathrm{C}$ language binding for the BSI services. This set of services consists of $23 \mathrm{C}$ functions derived from the pseudo IDL specification (Chapter 4). The return codes for the functions are as defined in Section 4.4. The $\mathrm{C}$ binding is grouped into three functional modules as follows:

- A Smart Card Utility Provider Module

- A Smart Card Generic Container Provider Module

- A Smart Card Cryptographic Provider Module

\section{E.1 Type Definitions for BSI Functions}

The following type definitions are used by multiple BSI functions.

$$
\begin{array}{lll}
\text { \#typedef } & \text { unsigned long } & \text { UTILCardHandle } \\
\text { \#typedef } & \text { unsigned char } & \text { GCtag }
\end{array}
$$

\section{E.2 Parameter Format and Buffer Size Discovery Process}

Many BSI function calls accept and/or return variable-length string data. The buffers that store the strings are paired with an integer value representing the number of bytes (the size of the buffer). This number includes the additional byte for the NULL terminator in the case where actual text is expected (e.g. Reader Name). Calling applications shall allocate buffers of sufficient size to hold string arguments returned by the BSI functions. The BSI shall provide a discovery mechanism to allow applications to determine required buffer size for returned data. To determine the required buffer size, the calling application must typically call the BSI function two times. The first time to get the required buffer size (discovery call), and the second time to execute the function with the correct buffer size (execution call). However, only one call is possible if the client application is able to estimate the required buffer size. In that last case, the call is an execution call.

The client application sets the pointer to the buffer that should be allocated for the returned arguments to NULL. This approach signals to the service that it must determine the buffer size required for the returned arguments and return this information in the corresponding paired integers. The client application then allocates buffers of the required size, sets the paired integers accordingly, and calls the BSI function a second time. The SPS must check the length integer against its previously cached value and, if the value contained in the length integer is greater than or equal to the required buffer length, it shall return the appropriate data in the buffers. See Example 1 and 2 in Section E. 3 for additional information.

If an application knows or is able to estimate the required buffer size beforehand, it can shorten the process by making only one call. To do so, the application allocates buffers it believes to be of sufficient size to hold the data returned by the BSI function, sets the paired length integers accordingly, and calls the BSI function. The SPS shall check the length integer against the required value and, if it is greater than or equal to the required buffer length, it shall return the appropriate data in the buffers. If not, the BSI function shall return the BSI_INSUFFICIENT_BUFFER error code and the required buffer sizes in the respective paired length integers. See Example 3 in the Section E.3 for more information.

\section{E.2.1 Variable Length String Data}

Ten BSI function calls accept and/or return variable-length string data identified in Table E-1. 
Table E-1: BSI functions using the discovery method

\begin{tabular}{|c|c|c|}
\hline BSI function & Discovery buffer & Discovery length \\
\hline gscBsiUtilGetVersion () & *uszVersion & *unVersionLen \\
\hline gscBsiUtilGetCardProperties () & *uszCCCUniqueID & *unCCCUniqueIDLen \\
\hline gscBsiUtilGetReaderList () & *uszReaderList & *unReaderListLen \\
\hline $\begin{array}{l}\text { gscBsiUtilPassthru () (See Note in } \\
\text { Section E.3.9) }\end{array}$ & *uszCardResponse & *unCardResponseLen \\
\hline gscBsiGcReadTagList () & *TagArray & *unNbTags \\
\hline gscBsiGcReadValue () & *uszValue & *unValueLen \\
\hline gscBsiGetChallenge () & *uszChallenge & *unChallengeLen \\
\hline gscBsiSkiInternalAuthenticate () & *uszCryptogram & *unCryptogramLen \\
\hline gscBsiPkiCompute () & *uszResult & *unResultLen \\
\hline gscBsiPkiGetCertificate () & *uszCertificate & *unCertificateLen \\
\hline
\end{tabular}

Each of these functions is invoked in the discovery mode by passing in a NULL value for the discovery buffer parameter. With the exception of gscBsiGcReadTagList (), each of these returns (Discovery call) the size in bytes (including the NULL Terminator) of the buffer needed to store the return variablelength string data. The lone exception, gscBsiGcReadTagList (), returns the number of tags in the tag array, so that the size of the array buffer needed is given by "*unNbTags * size of (GCtag)".

\section{E.3 Discovery Mechanisms Code Samples}

Following are three examples in $\mathrm{C}$ illustrating the discovery mechanism.

The three examples make the following assumptions:

- Application defined return codes SUCCESS \& FAILURE

- ERROR_RETURN reports error and returns FAILURE

- Parameters AID and AID length are given

- PROCESS_READ_CERTIFICATE processes the read of the certificate

\section{Example 1}

\section{\{}

// Discovers the correct size for the certificate buffer, allocates memory and executes.

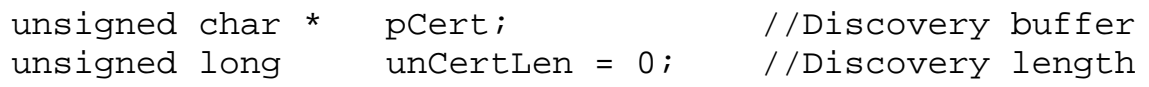




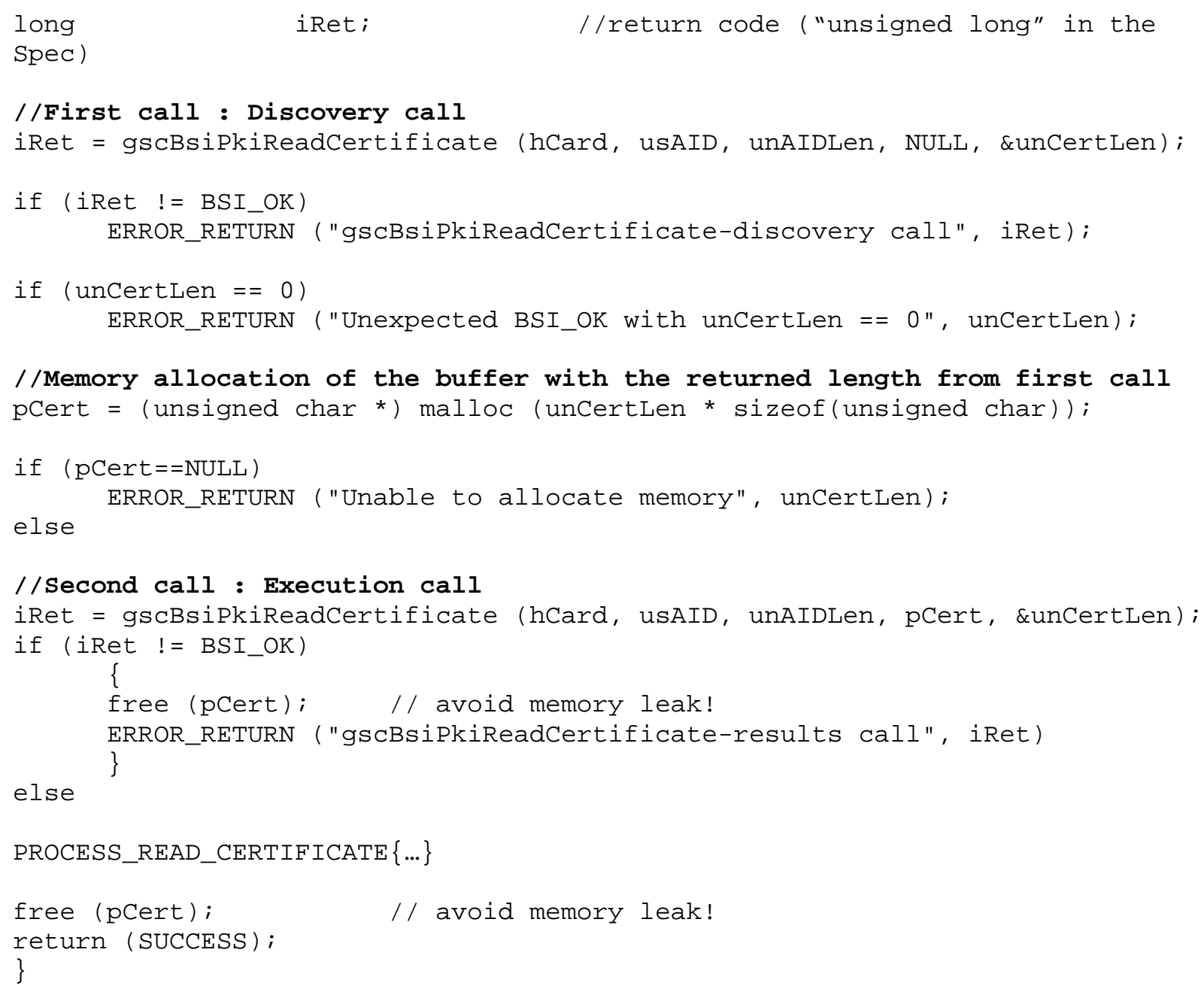


NIST IR 6887 - 2003 EDITION, GSC-IS VERSION 2.1

\section{Example 2}

\{

// Try default buffer first, if buffer is large enough normal execution occurs, or if buffer is too small reacts by discovering the length and executes.

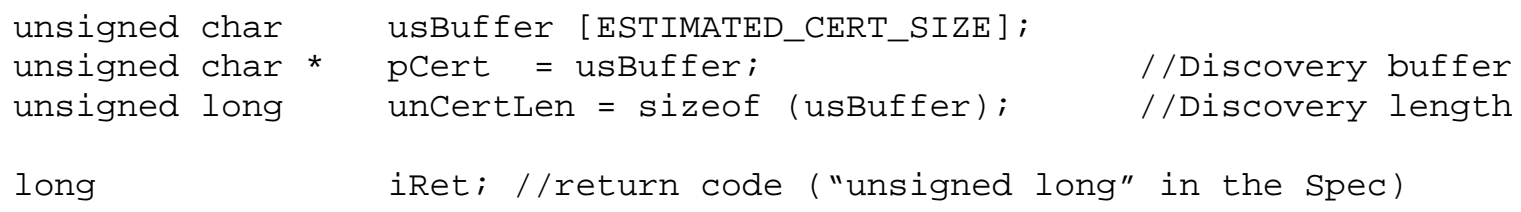




\section{Example 3}

\{

// Use a buffer so large that discovery is never necessary.

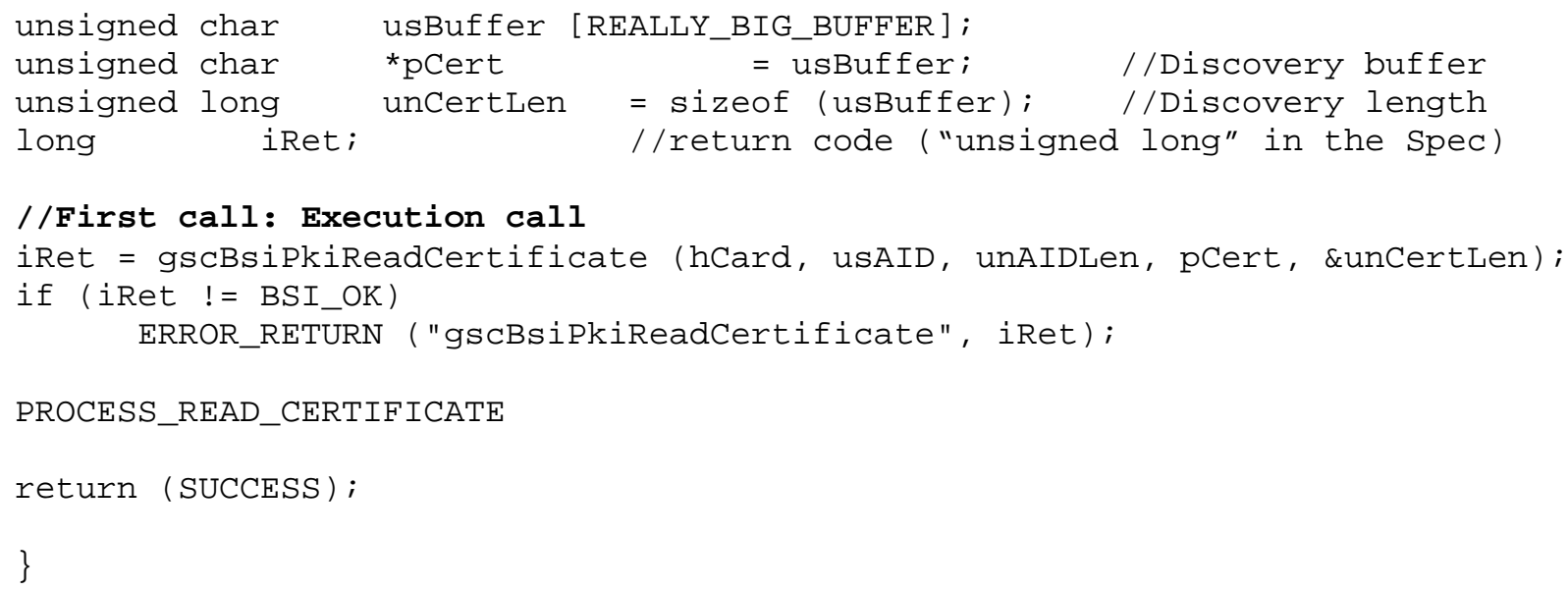




\section{E.4 Smart Card Utility Provider Module Interface Definition}

\section{E.4.1 gscBsiUtilAcquireContext()}

Purpose: $\quad$ This function shall establish a session with a target container on the smart card by submitting the appropriate Authenticator in the BSIAuthenticator structure. For ACRs requiring external authentication (XАUTH), the uszAuthValue field of the BSIAuthenticator structure must contain a cryptogram calculated by encrypting a random challenge from gscBsiGetChallenge (). In cases where the card acceptance device authenticates the smart card, this function returns a BSI_TERMINAL_AUTH return code and the cryptogram is ignored.

For ACRs that require chained authentication such as BSI_ACR_PIN_AND_XAUTH, the calling application passes in the required authenticators in multiple BSIAuthenticator structures. In this example the calling application passes a PIN and the appropriate External Authentication cryptogram in two BSIAuthenticator structures. The client application must set the unAccessMethodType field of each BSIAuthenticator structure to match the type of authenticator contained in the structure. To satisfy an ACR of BSI_ACR_PIN_AND_XAUTH, the application would construct a sequence of two BSIAuthenticators: one containing a PIN and one containing an External Authentication cryptogram. The BSIAuthenticator structure containing the PIN would have an unAccessMethodType of BSI_AM_PIN, and the BSIAuthenticator structure containing the External Authentication cryptogram would have an unAccessMethodType of BSI_AM_XAUTH.

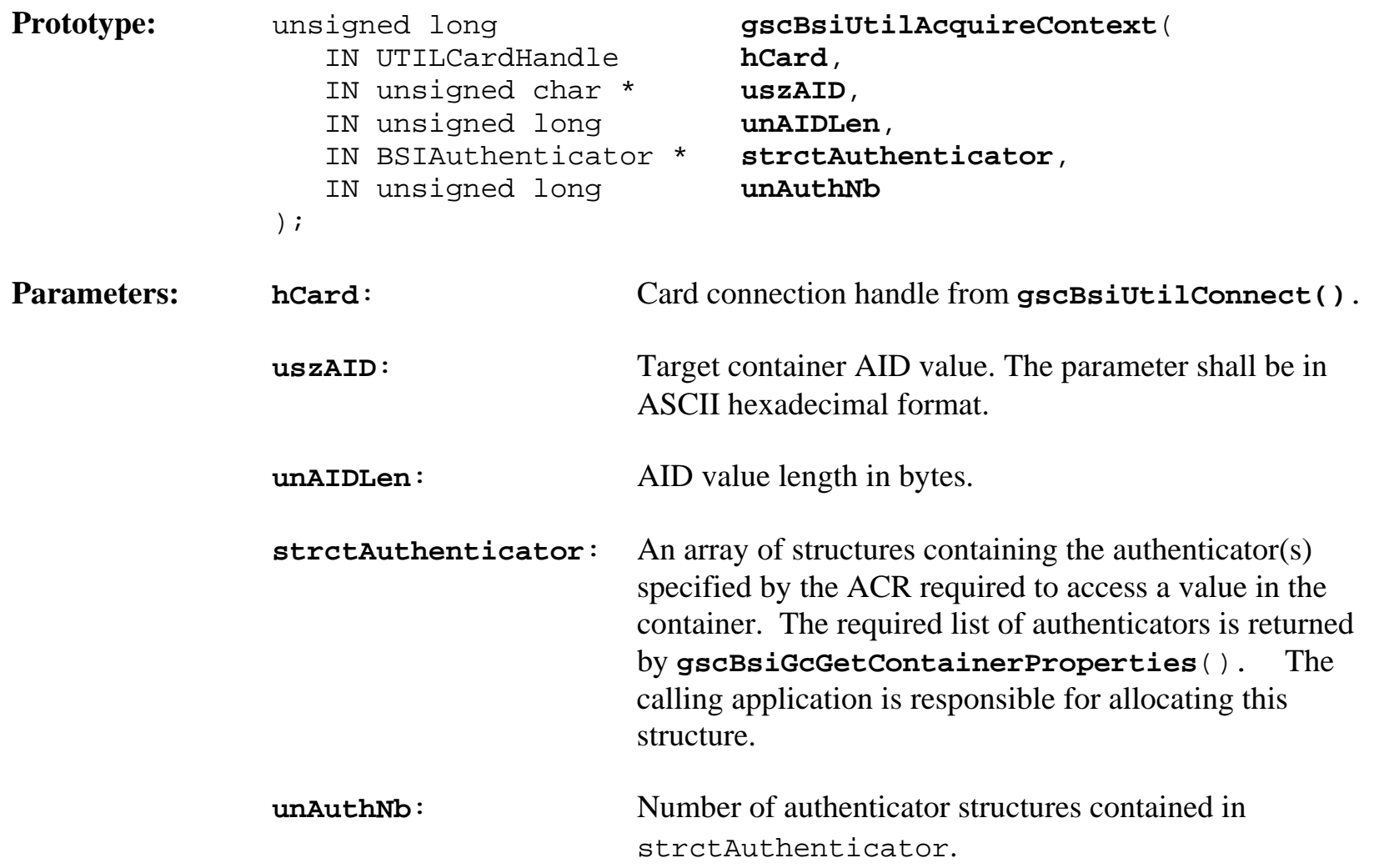


The BSIAuthenticator structure is defined as follows.

BSI_AUTHENTICATOR_MAX_LEN and BSI_KEY_LENGTH are implementationdependent constants.

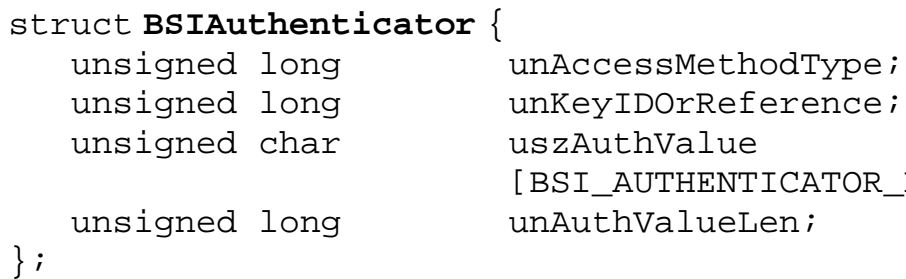

Variables associated with the BSIAuthenticator structure:

unAccessMethodType: Access Method Type (see Table 3-1 in Section 3.1).

unKeyIDOrReference: Key identifier or reference of the authenticator. This is used to distinguish between multiple authenticators with the same Access Method Type.

uszAuthValue:

Authenticator, can be an external authentication cryptogram or PIN. If the authenticator value is NULL, then BSI is in charge of gathering authentication information and authenticating to the card.

unAuthValueLen: Authenticator value length in bytes.

$\begin{array}{ll}\text { Return Codes: } & \text { BSI_OK } \\ & \text { BSI_BAD_HANDLE } \\ & \text { BSI_BAD_AID } \\ & \text { BSI_BAD_PARAM } \\ & \text { BSI_ACR_NOT_AVAILABLE } \\ & \text { BSI_BAD_AUTH } \\ & \text { BSI_CARD_REMOVED } \\ & \text { BSI_SC_LOCKED } \\ & \text { BSI_PIN_BLOCKED } \\ & \text { BSI_TERMINAL_AUTH } \\ & \text { BSI_UNKNOWN_ERROR }\end{array}$




\section{E.4.2 gscBsiUtilConnect()}

Purpose: $\quad$ Establish a logical connection with the card in a specified reader.

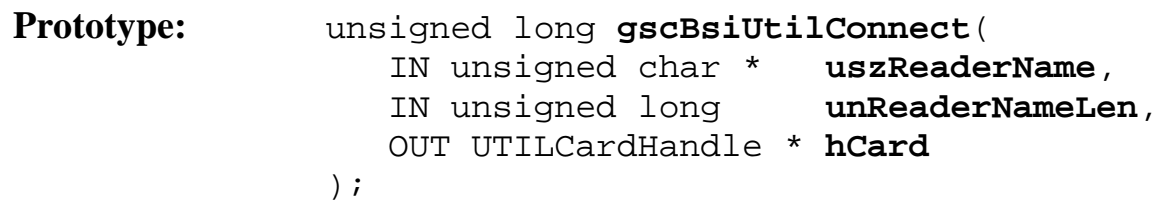
is a NULL pointer, the SPS shall attempt to connect to the card in the first available reader, as returned by a call to the BSI's function gscBsiUtilGetReaderList (). The reader name string shall be stored as ASCII encoding String. (See Section 4.2)

unReaderNameLen: Length of the reader name in bytes.

$\begin{array}{ll}\text { Return Codes: } & \text { BSI_OK } \\ & \text { BSI_BAD_PARAM } \\ & \text { BSI_UNKNOWN_READER } \\ & \text { BSI_CARD_ABSENT } \\ & \text { BSI_TIMEOUT_ERROR } \\ & \text { BSI_UNKNOWN_ERROR }\end{array}$




\section{E.4.3 gscBsiUtilDisconnect()}

Purpose: $\quad$ Terminate a logical connection to a card.

Prototype: $\quad$ unsigned long gscBsiutilDisconnect (

IN UTILCardHandle hCard

$$
\text { ) ; }
$$

Parameters: hCard: $\quad$ Card connection handle from gscBsiutilconnect ().

Return Codes: $\quad$ BSI_OK

BSI_BAD_HANDLE

BSI_CARD_REMOVED

BSI_UNKNOWN_ERROR 


\section{E.4.4 gscBsiUtilBeginTransaction()}

Purpose:

Prototype:

Parameters: hCard:

blType:

Return Codes:
Starts an exclusive transaction with the smart card referenced by hCard. When the transaction starts, all other applications are precluded from accessing the smart card while the transaction is in progress. Two types of calls can be made with that function: a blocking transaction call and a non-blocking transaction call. A boolean type parameter identify which mode is called. In the non-blocking mode, the call will return immediately if another client has an active transaction lock. The returned error code will be BSI_SC_LOCKED. In the blocking mode, the call will wait indefinitely for any active transaction locks to be released. A transaction must be completed by a call to gscBsiutilendTransaction() .

For single-threaded BSI implementations, it can be assumed that each application will be associated with a separate process. The same process that starts a transaction must also complete the transaction. For multi-threaded BSI implementations, it can be assumed that each application will be associated with a separate thread and/or process. The same thread that starts a transaction must also complete the transaction.

If this function is called by a thread that has already called gscBsiutilBeginTransaction() but has not yet called gscBsiUtilEndTransaction() it will return the error BSI_NOT_TRANSACTED.

If the SPS (Service Provider Software) does not support transaction locking, it should return the error code BSI_NO_SPSSERVICE in response to a call to

gscBsiutilBeginTransaction().

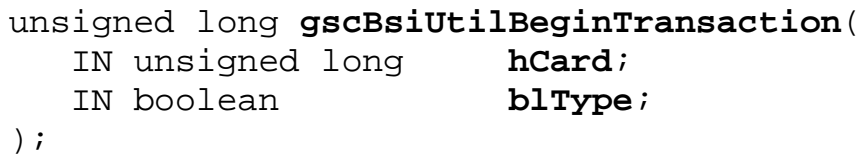

Card communication handle returned from gscBsiutilconnect ()

Boolean specifying the type of transaction call ( blType set to "true" in blocking mode. blType set to "false" in non-blocking mode).

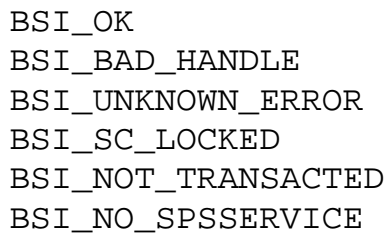




\section{E.4.5 gscBsiUtilEndTransaction()}

Purpose: $\quad$ Completes a previously started transaction, allowing other applications to resume interactions with the card.

If this function is called by a thread that has not yet called gscBsiutilBeginTransaction() it will return the error BSI_NOT_TRANSACTED.

If the SPS (Service Provider Software) does not support transaction locking, it should return the error code BSI_NO_SPSSERVICE in response to a call to gscBsiutilendTransaction().

Prototype: $\quad$ unsigned long gscBsiutilendTransaction( IN unsigned long hCard

) ;

Parameters: hCard:

Card communication handle returned from gscBsiutilConnect ().

Return Codes: $\quad$ BSI_OK

BSI_BAD_HANDLE

BSI_UNKNOWN_ERROR

BSI_NOT_TRANSACTED

BSI_NO_SPSSERVICE 


\section{E.4.6 gscBsiUtilGetVersion()}

Purpose: $\quad$ Returns the BSI implementation version.

\begin{tabular}{|c|c|c|c|c|}
\hline Prototype: & unsigned & long gscBsiuti & LiGet & tVersion ( \\
\hline & INOUT & unsigned char & $\star$ & uszVersion, \\
\hline & INOUT & unsigned long & * & punversionLen \\
\hline
\end{tabular}

Parameters: uszVersion: The BSI and SPS version formatted as "major,minor,revision, build_numberl0". The version text shall be stored as ASCII encoded String. (See Section 4.2)

punVersionLen: $\quad$ Length of the version string.

$\begin{array}{ll}\text { Return Codes: } & \text { BSI_OK } \\ & \text { BSI_BAD_PARAM } \\ & \text { BSI_INSUFFICIENT_BUFFER } \\ & \text { BSI_UNKNOWN_ERROR }\end{array}$

Discovery Mode:

Parameters: uszVersion: Set to NULL.

punVersionLen: $\quad$ Pointer to value containing the required buffer length to contain the version string, including a null terminator.

$\begin{array}{ll}\text { Return Codes: } & \text { BSI_OK } \\ & \text { BSI_BAD_PARAM } \\ & \text { BSI_UNKNOWN_ERROR }\end{array}$




\section{E.4.7 gscBsiUtilGetCardProperties()}

Purpose: $\quad$ Retrieves ID and capability information for the card.

\begin{tabular}{|c|c|c|c|}
\hline \multirow[t]{5}{*}{ Prototype: } & \multicolumn{3}{|c|}{ unsigned long gscBsiUtilGetCardProperties ( } \\
\hline & IN UTI & ILCardHandle & hCard, \\
\hline & INOUT & unsigned char * & uszCCCUniqueID, \\
\hline & INOUT & unsigned long * & punCCCUniqueIDLen, \\
\hline & OUT un & hsigned long * & punCardCapability \\
\hline
\end{tabular}

Parameters: hCard:

uszCCCUniqueID :

punCCCUniqueIDLen:

punCardCapability:
Card connection handle from gscBsiutilConnect () .

Buffer for the Card Capability Container ID, represented in ASCII Hexadecimal.

Length of the CCC Unique ID string (input). Length of the returned Card Unique ID string including the null terminator (output).

Bit mask value defining the providers supported by the card. The bit masks represent the Generic Container Data Model, the Symmetric Key Interface, and the Public Key Interface providers respectively:

$\begin{array}{ll}\text { \#define BSI_GCCDM } & 0 \times 00000001 \\ \text { \#define BSI_SKI } & 0 \times 00000002 \\ \text { \#define BSI_PKI } & 0 \times 00000004\end{array}$

Return Codes: $\quad$ BSI_OK

BSI_BAD_HANDLE

BSI_CARD_REMOVED

BSI_SC_LOCKED

BSI_BAD_PARAM

BSI_INSUFFICIENT_BUFFER

BSI_NO_CARDSERVICE

BSI_UNKNOWN_ERROR

Discovery Mode:

Parameters: hCard: $\quad$ Card connection handle from gscBsiutilConnect ().

uszCCCUniqueID: $\quad$ Set to NULL.

punCCCUniqueIDLen: Pointer to value containing the required buffer length for the CCC Unique ID string, including a null terminator.

punCardCapability: Can be set to NULL, unused in discovery. 
NIST IR 6887 - 2003 EDITION, GSC-IS VERSION 2.1

Return Codes : $\quad$ BSI_OK

BSI_BAD_HANDLE

BSI_CARD_REMOVED

BSI_SC_LOCKED

BSI_BAD_PARAM

BSI_UNKNOWN_ERROR 


\section{E.4.8 gscBsiUtilGetCardStatus()}

Purpose: $\quad$ Checks whether a given card handle is associated with a card that is inserted into a powered up reader.

Prototype: unsigned long gscBsiUtilGetCardstatus ( IN UTILCardHandle hCard ) ;

Parameters: hCard: Card connection handle from gscBsiutilconnect ().

Return Codes: BSI_OK

BSI_BAD_HANDLE

BSI_CARD_REMOVED

BSI_UNKNOWN_ERROR 


\section{E.4.9 gscBsiUtilGetExtendedErrorText()}

Purpose: $\quad$ When a BSI function call returns an error, an application can make a subsequent call to this function to receive additional implementation specific error information, if available.

Prototype:

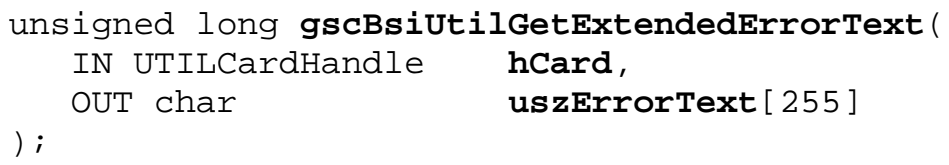

Parameters: hCard: $\quad$ Card connection handle gscBsiutilconnect ().

uszErrorText : $\quad$ A fixed length buffer containing an implementation specific error text string. The text string is nullterminated, and has a maximum length of 255 characters including the null terminator. The calling application must allocate a buffer of 255 bytes. If an extended error text string is not available, this function returns a NULL string and BSI_NO_TEXT_AVAILABLE. The error text shall be stored as ASCII encoding String. (See Section 4.2)

$\begin{array}{ll}\text { Return Codes: } & \text { BSI_OK } \\ & \text { BSI_BAD_HANDLE } \\ & \text { BSI_NO_TEXT_AVAILABLE } \\ & \text { BSI_UNKNOWN_ERROR }\end{array}$




\section{E.4.10 gscBsiUtilGetReaderList()}

Purpose: $\quad$ Retrieves the list of available readers.

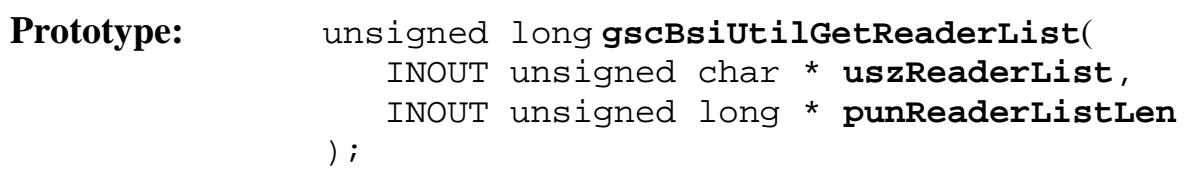

Parameters: uszReaderList: $\quad$ Reader list buffer. The reader list is returned as a multistring, each reader name terminated by a ' 10 '. The list itself is terminated by an additional trailing ' 10 ' character.

punReaderListLen: $\quad$ Reader list length in bytes including all terminating ' 10 ' characters.

$\begin{array}{ll}\text { Return Codes: } & \text { BSI_OK } \\ & \text { BSI_BAD_PARAM } \\ & \text { BSI_INSUFFICIENT_BUFFER } \\ & \text { BSI_UNKNOWN_ERROR }\end{array}$

Discovery Mode:

Parameters: uszReaderList: $\quad$ Set to NULL.

punReaderListLen: $\quad$ Required buffer length for Reader list in bytes including all terminating ' 10 ' characters.

$\begin{array}{ll}\text { Return Codes: } & \text { BSI_OK } \\ & \text { BSI_BAD_PARAM } \\ & \text { BSI_UNKNOWN_ERROR }\end{array}$ 


\section{E.4.11 gscBsiUtilPassthru()}

Purpose: $\quad$ Allows a client application to send a "raw" APDU through the BSI directly to the card and receive the APDU-level response.

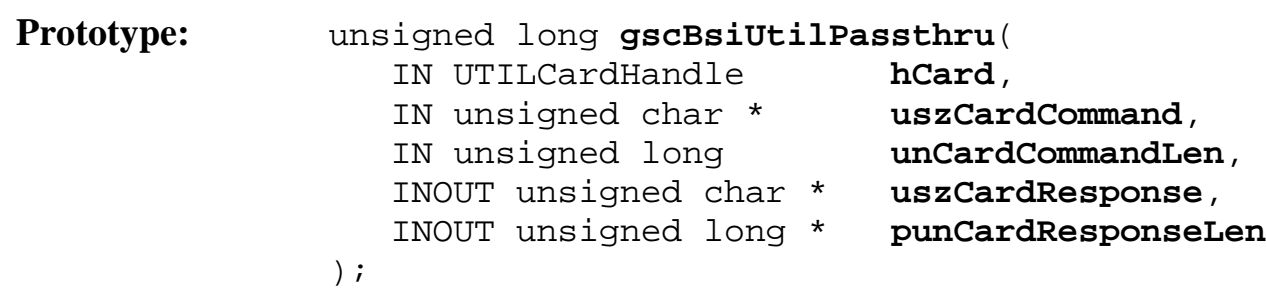

Parameters: hCard: $\quad$ Card connection handle from gscBsiutilconnect () .

uszCardCommand: $\quad$ The APDU to be sent to the card. That parameter must be in ASCII hexadecimal format.

unCardCommandLen: Length of the APDU string to be sent.

uzsCardResponse: $\quad$ Pre-allocated buffer for the APDU response from the card. The response must include the status bytes SW1 and SW2 returned by the card. If the size of the buffer is insufficient, the SPS shall return truncated response data and the return code BSI_INSUFFICIENT_BUFFER. That parameter must be in ASCII hexadecimal format.

punCardResponselen: Length of the APDU response. If the size of the uszCardResponse buffer is insufficient, the SPS shall return the correct size in this field.

$\begin{array}{ll}\text { Return Codes: } & \text { BSI_OK } \\ & \text { BSI_BAD_HANDLE } \\ & \text { BSI_BAD_PARAM } \\ & \text { BSI_INSUFFICIENT_BUFFER } \\ & \text { BSI_CARD_REMOVED } \\ & \text { BSI_SC_LOCKED } \\ & \text { BSI_UNKNOWN_ERROR }\end{array}$

\section{Discovery Mode (depending on usage):}

Note: The discovery mechanism may cause the command APDU to be executed twice depending on the context of use.

The discovery mode is as follows:

Parameters: hCard: Card connection handle from gscBsiutilconnect ().

uszCardCommand: The APDU to be sent to the card. 
unCardCommandLen: Length of the APDU string to be sent.

uzsCardResponse: Set to NULL.

punCardResponseLen: Length of the buffer required to contain the APDU response.

$\begin{array}{ll}\text { Return Codes: } & \text { BSI_OK } \\ & \text { BSI_BAD_HANDLE } \\ & \text { BSI_SC_LOCKED } \\ & \text { BSI_BAD_PARAM } \\ & \text { BSI_CARD_REMOVED } \\ & \text { BSI_UNKNOWN_ERROR }\end{array}$




\section{E.4.12 gscBsiUtilReleaseContext()}

Purpose: $\quad$ Terminate a session with the target container on the card.

Prototype: unsigned long gscBsiutilReleasecontext (
IN UTILCardHandle hCard,
IN unsigned char * uszAID,
IN unsigned long
) $;$

Parameters: hCard: Card connection handle from gscBsiutilconnect ().

USzAID :

UnAIDLen:

Return Codes: $\quad$ BSI_OK

BSI_BAD_HANDLE

BSI_BAD_AID

BSI_BAD_PARAM

BSI_CARD_REMOVED

BSI_SC_LOCKED

BSI_UNKNOWN_ERROR
Target container AID value. The parameter shall be in ASCII hexadecimal format.

AID value length in bytes. 


\section{E.5 Smart Card Generic Container Provider Module Interface Definition}

\section{E.5.1 gscBsiGcDataCreate()}

Purpose: $\quad$ Create a new data item in $\{$ Tag, Length, Value $\}$ format in the selected container.

Prototype:

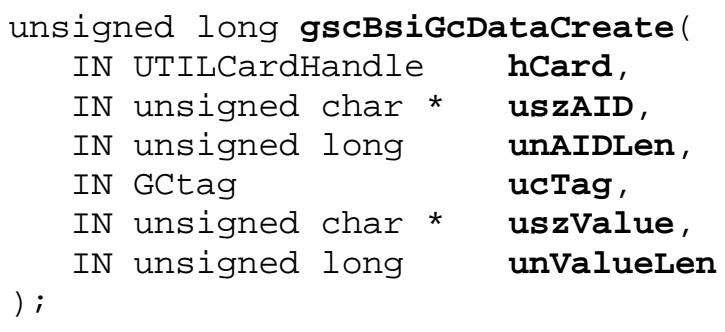

\section{Parameters: hcard:}

uszAID :

UnAIDLen:

ucTag :

uszValue :

unValueLen:

Return Codes:

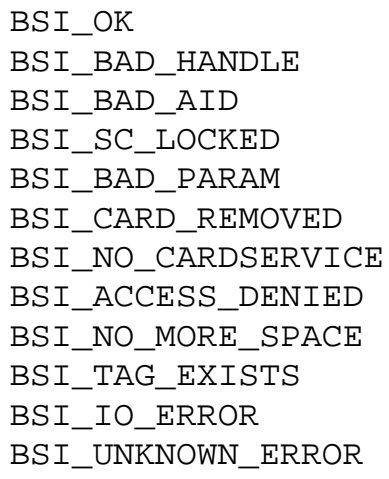

Card connection handle from gscBsiutilconnect ().

Target container AID value. The parameter shall be in ASCII hexadecimal format.

AID value length in bytes.

Tag of data item to store.

Data value to store.

Data value length in bytes. 


\section{E.5.2 gscBsiGcDataDelete()}

Purpose: $\quad$ Delete the data item associated with the tag value in the specified container.

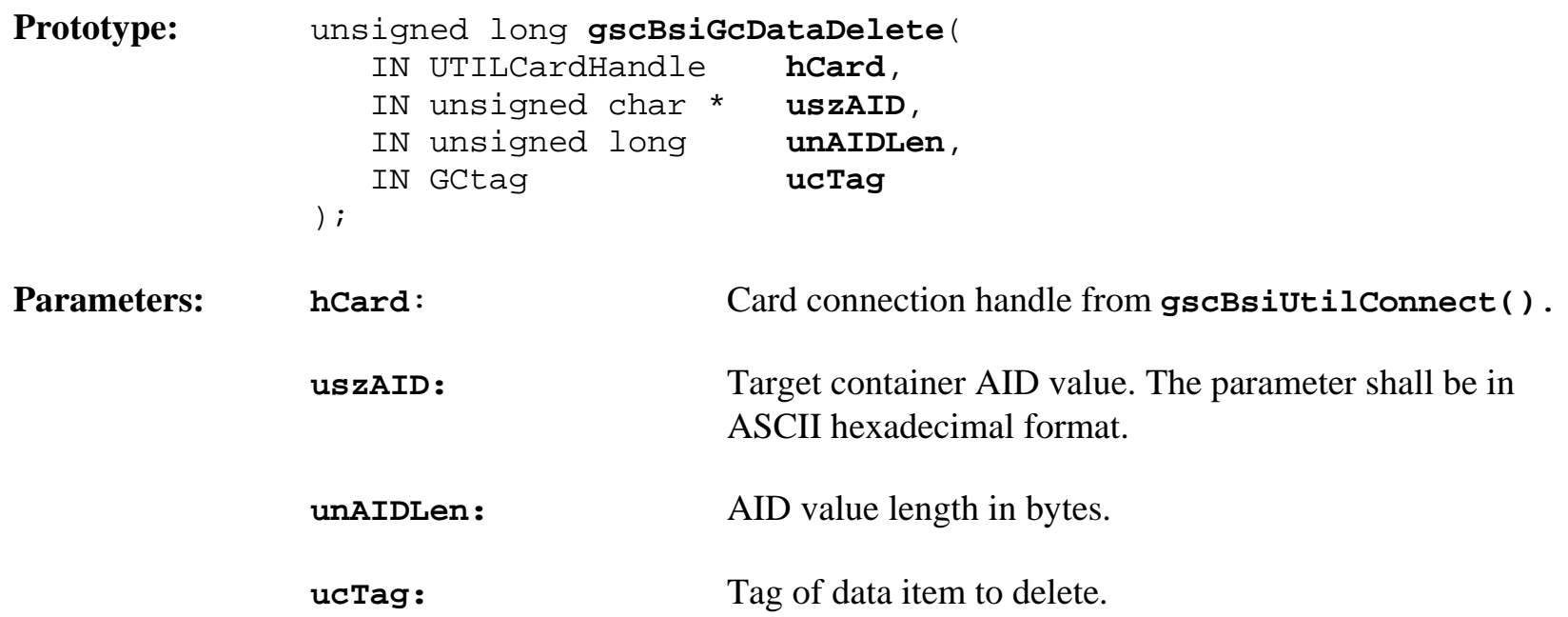

Return Codes: $\quad$ BSI_OK

BSI_BAD_HANDLE

BSI_SC_LOCKED

BSI_BAD_AID

BSI_BAD_PARAM

BSI_BAD_TAG

BSI_CARD_REMOVED

BSI_NO_CARDSERVICE

BSI_ACCESS_DENIED

BSI_IO_ERROR

BSI_UNKNOWN_ERROR 


\section{E.5.3 gscBsiGcGetContainerProperties()}

Purpose: Retrieves the properties of the specified container.

Prototype:

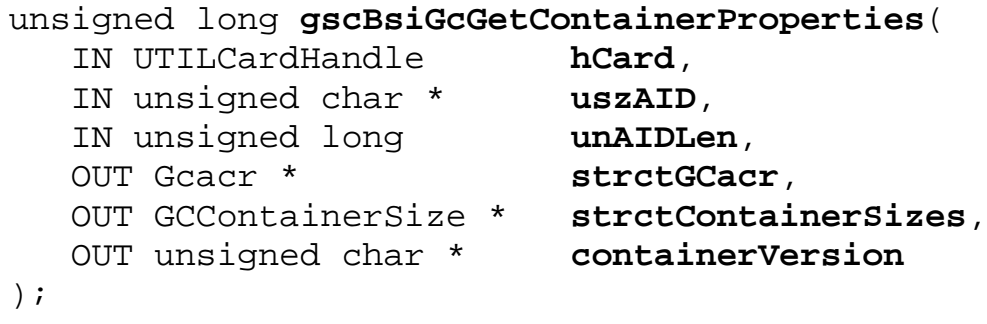

\section{Parameters: hCard:}

USzAID :

UnAIDLen :

strctGCacr :
Card connection handle from gscBsiutilConnect () .

Target container AID value. The parameter shall be in ASCII hexadecimal format.

AID value length in bytes.

Structure indicating access control conditions for all operations. The range of possible values for the members of this structure is defined in Table 3-2 (Section 3.1). The allowable ACRs for each function are listed in Table 3-3. unKey IDOrReference contains the key identifier or reference for each access method contained in the ACR in order of appearance. unAuthNb is the number of access methods logically combined in the ACR. ACRID is RFU and must be NULL (0x00) in this version.

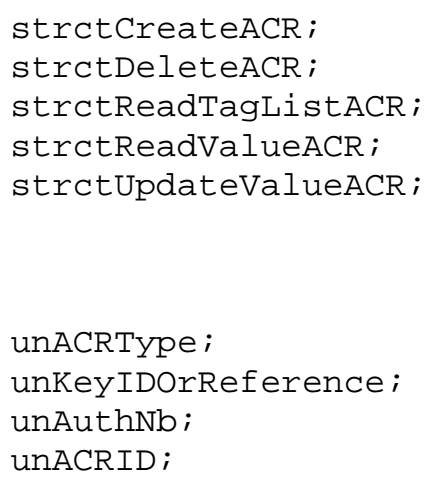

strctContainersizes: For Virtual Machine cards, the size(in bytes) of the container specified by uszAID. unMaxNbDataItems is the size of the T-Buffer, and

unMaxValuestorageSize is the size of the V-Buffer. For file system cards that cannot calculate these values, both fields of this structure will be set to 0 . 
NIST IR 6887 - 2003 EDITION, GSC-IS VERSION 2.1

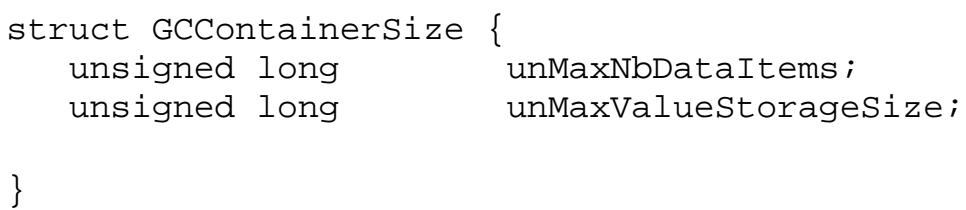

containerVersion: Version of the container. The format of this value is application dependent. In cases where the card cannot return a container version, this string will contain only the null terminator " 10 ".

Return Codes: $\quad$ BSI_OK
BSI_BAD_HANDLE
BSI_SC_LOCKED
BSI_BAD_AID
BSI_BAD_PARAM
BSI_CARD_REMOVED
BSI_NO_CARDSERVICE
BSI_UNKNOWN_ERROR 


\section{E.5.4 gscBsiGcReadTagList()}

Purpose: $\quad$ Return the list of tags in the selected container.

Prototype:

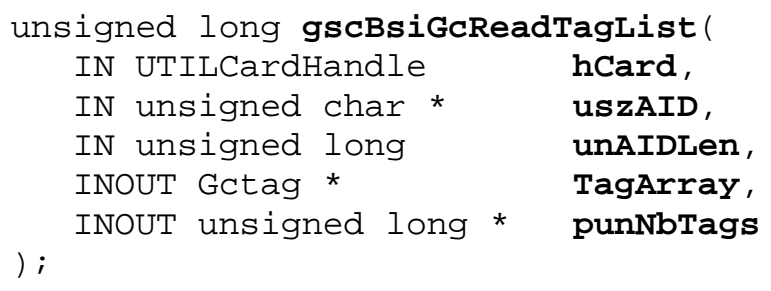

Parameters: hCard: Card connection handle from gscBsiutilconnect () .

USZAID :

UnAIDLen:

TagArray :

punNbTags :
Target container AID value. The parameter shall be in ASCII hexadecimal format.

AID value length in bytes.

An array containing the list of tags for the selected container.

Number of tags in TagArray.

Return Codes:

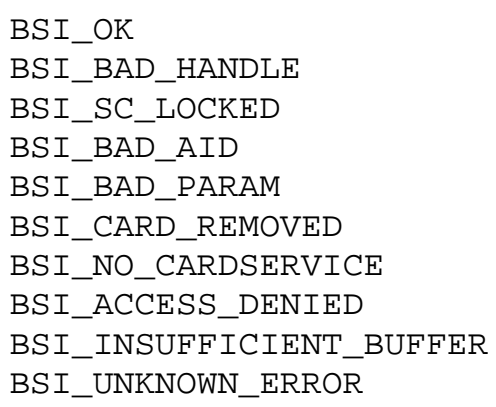

Discovery Mode:

Parameters: hCard:

uszAID :

UnAIDLen:

TagArray:

punNbTags :

Return Codes:

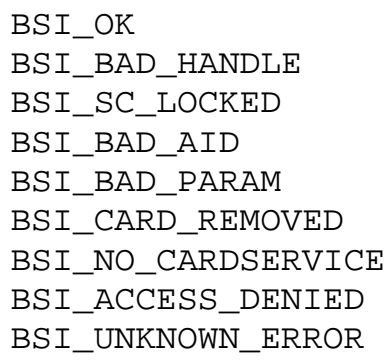

Card connection handle from gscBsiutilconnect () . Target container AID value.

AID value length in bytes.

Set to NULL.

Number of tags which would be contained in a resulting TagArray. 


\section{E.5.5 gscBsiGcReadValue()}

Purpose: $\quad$ Returns the Value associated with the specified Tag.

$\begin{array}{lll}\text { Prototype: } & \text { unsigned long } & \text { gscBsigcReadValue( } \\ \text { IN UTILCardHandle } & \text { hCard, } \\ \text { IN unsigned char * } & \text { uszAID, } \\ \text { IN unsigned long } & \text { unAIDLen, } \\ \text { IN GCtag } & \text { ucTag, } \\ \text { INOUT unsigned char * } & \text { uszValue, } \\ \text { INOUT unsigned long * } & \text { punvaluelen } \\ \text { ) } ; & \end{array}$

Parameters: hCard: $\quad$ Card connection handle from gscBsiutilconnect ().

USzAID :

UnAIDLen:

ucTag:

uszValue :

punValueLen:
Target container AID value. The parameter shall be in ASCII hexadecimal format.

AID value length in bytes.

Tag value of data item to read.

Value associated with the specified tag. The caller must allocate the buffer.

Size of the buffer allocated by the caller to hold the returned Value (input). Size of the Value returned (output).

Return Codes:

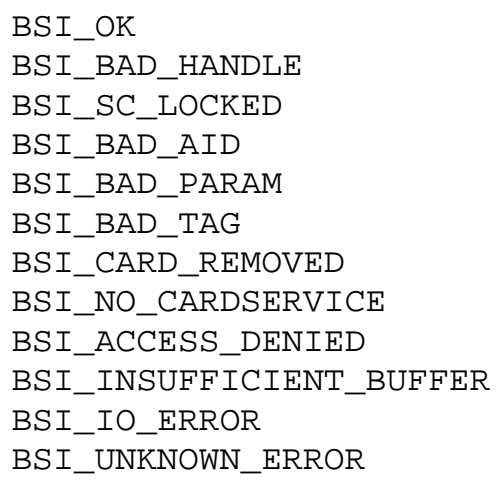

\section{Discovery Mode:}
Parameters: hCard:
Card connection handle from gscBsiutilConnect () .
UszAID :
Target container AID value.
UnAIDLen:
AID value length in bytes. 
ucTag :

uszValue:

punvaluelen:

Return Codes:
BSI_OK
BSI_BAD_HANDLE
BSI_BAD_AID
BSI_BAD_PARAM
BSI_SC_LOCKED
BSI_BAD_TAG
BSI_CARD_REMOVED
BSI_NO_CARDSERVICE
BSI_ACCESS_DENIED
BSI_IO_ERROR
BSI_UNKNOWN_ERROR

Tag value of data item to read.

Set to NULL.

Size of the buffer required to hold the returned Value. 


\section{E.5.6 gscBsiGcUpdateValue()}

Purpose: $\quad$ Updates the Value associated with the specified Tag.

$\begin{array}{rll}\text { Prototype: } & \text { unsigned long } & \text { gscBsiGcUpdatevalue ( } \\ \text { IN UTILCardHandle } & \text { hCard, } \\ \text { IN unsigned char * } & \text { uszAID, } \\ \text { IN unsigned long } & \text { unAIDLen, } \\ \text { IN GCtag } & \text { ucTag, } \\ \text { IN unsigned char * } & \text { uszValue, } \\ \text { IN unsigned long } & \text { unvaluelen } \\ \text { ); } & \end{array}$
Parameters: hCard: Card connection handle from gscBsiutilconnect () .
USZAID :
UnAIDLen:
ucTag :
uszValue:
unValueLen :
Target container AID value. The parameter shall be in ASCII hexadecimal format.
AID value length in bytes.
Tag of data item to update.
New Value of the data item.
Length in bytes of the new Value.

Return Codes:

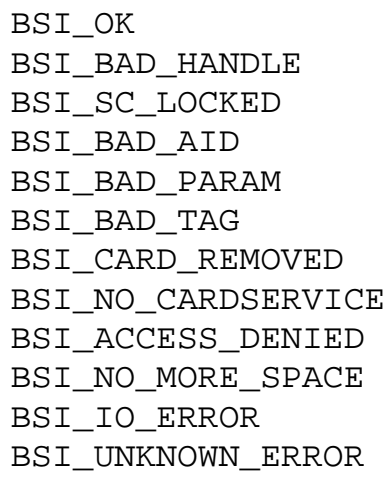




\section{E.6 Smart Card Cryptographic Provider Module Interface Definition}

\section{E.6.1 gscBsiGetChallenge()}

Purpose: $\quad$ Retrieves a randomly generated challenge from the card as the first step of a challenge-response authentication protocol between the client application and the card. The client subsequently encrypts the challenge using a symmetric key and returns the encrypted random challenge to the card through a call to gscBsiUtilAcquireContext () in the uszAuthValue field of a BSIAuthenticator structure.

Prototype:

unsigned long

IN UTILCardHandle

IN unsigned char *

IN unsigned long

INOUT unsigned char *

INOUT unsigned long *

) ;

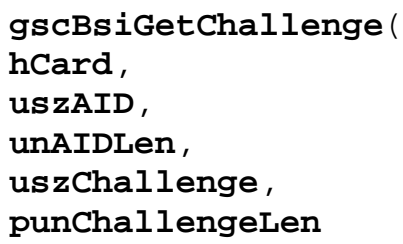

Parameters: hCard:

Card connection handle from gscBsiutilconnect () .

UszAID :

Target container AID value. The parameter shall be in ASCII hexadecimal format.

unAIDLen :

uszChallenge:

punChallengelen: Length of random challenge in bytes.
AID value length in bytes.

Random challenge returned from the card.

$\begin{array}{ll}\text { Return Codes: } & \text { BSI_OK } \\ & \text { BSI_BAD_HANDLE } \\ & \text { BSI_SC_LOCKED } \\ & \text { BSI_BAD_AID } \\ & \text { BSI_BAD_PARAM } \\ & \text { BSI_CARD_REMOVED } \\ & \text { BSI_NO_CARDSERVICE } \\ & \text { BSI_INSUFFICIENT_BUFFER } \\ & \text { BSI_UNKNOWN_ERROR }\end{array}$

Discovery Mode:

Parameters: hCard: $\quad$ Card connection handle from gscBsiutilconnect () .

USzAID:

UnAIDLen: 
NIST IR 6887 - 2003 EDITION, GSC-IS VERSION 2.1

uszChallenge:

punChallengelen:
Set to NULL.

Length of buffer required to store returned random challenge in bytes.

$\begin{array}{ll}\text { Return Codes: } & \text { BSI_OK } \\ & \text { BSI_BAD_HANDLE } \\ & \text { BSI_SC_LOCKED } \\ & \text { BSI_BAD_AID } \\ & \text { BSI_BAD_PARAM } \\ & \text { BSI_CARD_REMOVED } \\ & \text { BSI_NO_CARDSERVICE } \\ & \text { BSI_UNKNOWN_ERROR }\end{array}$




\section{E.6.2 gscBsiSkilnternalAuthenticate()}

Purpose: $\quad$ Computes a symmetric key cryptogram in response to a challenge. In cases where the card reader authenticates the card, this function does not return a cryptogram. In these cases a BSI_TERMINAL_AUTH will be returned if the card reader successfully authenticates the card. BSI_ACCESS_DENIED is returned if the card reader fails to authenticate the card.

$\begin{array}{lll}\text { Prototype: } & \text { gscBsiskiInternalAuthenticate } \\ \text { IN UTILCardHandle } & \text { hCard, } \\ \text { IN unsigned char* } & \text { uszAID, } \\ \text { IN unsigned long } & \text { unAIDLen, } \\ \text { IN unsigned char } & \text { ucAlgoID, } \\ \text { IN unsigned char* } & \text { uszChallenge, } \\ \text { IN unsigned long } & \text { unChallengelen, } \\ \text { INOUT unsigned char * } & \text { uszCryptogram, } \\ \text { INOUT unsigned long * } & \text { punCryptogramLen } \\ \text { ); } & \end{array}$

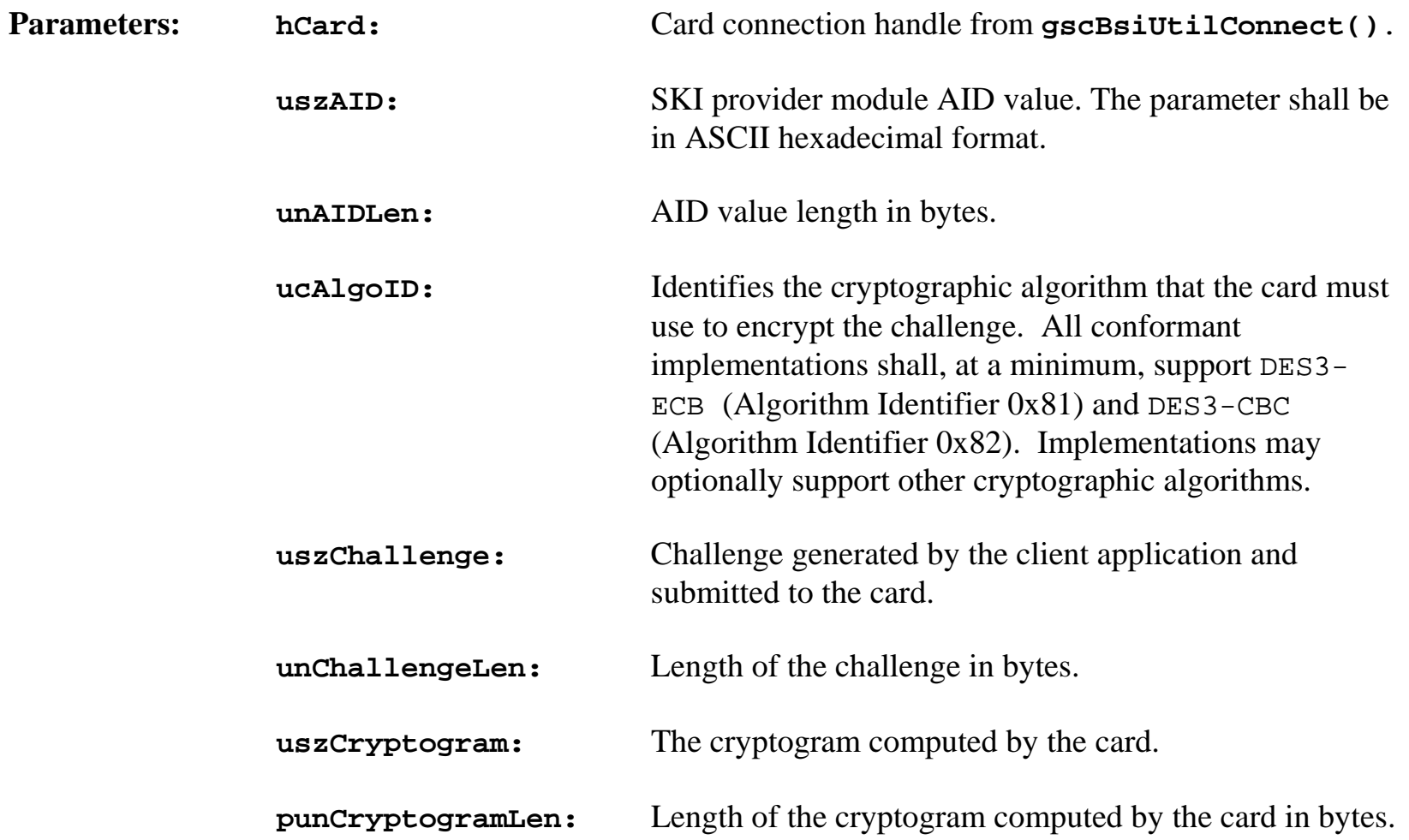

Return Codes: $\quad$ BSI_OK

BSI_BAD_HANDLE

BSI_SC_LOCKED

BSI_BAD_AID

BSI_BAD_PARAM

BSI_BAD_ALGO_ID

BSI_CARD_REMOVED

BSI_NO_CARDSERVICE

BSI_ACCESS_DENIED

BSI_TERMINAL_AUTH

BSI_INSUFFICIENT_BUFFER 


\section{Discovery Mode:}

BSI_UNKNOWN_ERROR

Parameters: hCard:

USZAID:

UnAIDLen:

ucAlgoID :

uszChallenge :

unChallengeLen:

uszCryptogram :

punCryptogramLen:
Card connection handle from gscBsiutilconnect () .

SKI provider module AID value.

AID value length in bytes.

Identifies the cryptographic algorithm that the card must use to encrypt the challenge. All conformant implementations shall, at a minimum, support DES3ECB (Algorithm Identifier 0x81) and DES3-CBC (Algorithm Identifier 0x82). Implementations may optionally support other cryptographic algorithms.

Challenge generated by the client application and submitted to the card.

Length of the challenge in bytes.

Set to NULL.

Length of the buffer required to store the cryptogram computed by the card in bytes.

\footnotetext{
Return Codes: $\quad$ BSI_OK

BSI_BAD_HANDLE

BSI_BAD_AID

BSI_BAD_PARAM

BSI_SC_LOCKED

BSI_BAD_ALGO_ID

BSI_CARD_REMOVED

BSI_NO_CARDSERVICE

BSI_ACCESS_DENIED

BSI_TERMINAL_AUTH

BSI_UNKNOWN_ERROR
} 


\section{E.6.3 gscBsiPkiCompute()}

Purpose: $\quad$ Performs a private key computation on the message digest using the private key associated with the specified AID.

Prototype:

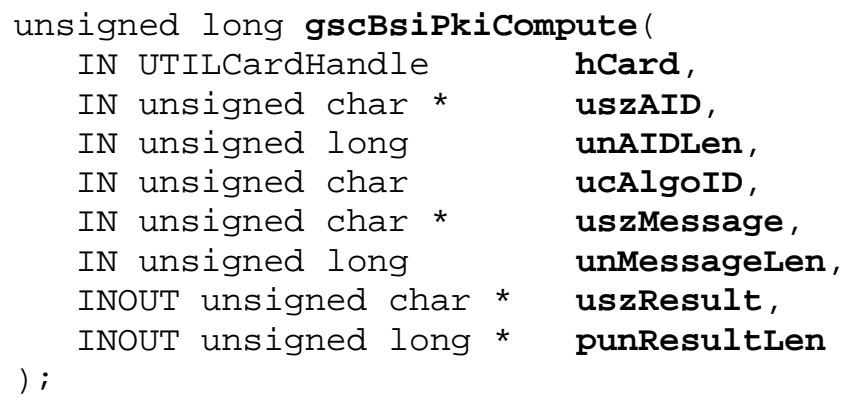

Parameters: hCard: $\quad$ Card connection handle from gscBsiutilConnect ().

UszAID: $\quad$ PKI provider module AID value. The parameter shall be in ASCII hexadecimal format.

unAIDLen: $\quad$ AID value length in bytes.

ucAlgoID: $\quad$ Identifies the cryptographic algorithm that will be used to generate the signature. All conformant implementations shall, at a minimum, support RSA_NO_PAD (Algorithm Identifier 0xA3). Implementations may optionally support other algorithms.

us zMessage :

The hash of the message to be signed.

unMessageLen:

Length of hashed message to be signed, in bytes.

uszResult :

Buffer containing the signature.

punResultLen: $\quad$ Length of the signature buffer in bytes.

Return Codes: $\quad$ BSI_OK

BSI_BAD_HANDLE

BSI_SC_LOCKED

BSI_BAD_AID

BSI_BAD_PARAM

BSI_BAD_ALGO_ID

BSI_CARD_REMOVED

BSI_ACCESS_DENIED

BSI_NO_CARDSERVICE

BSI_INSUFFICIENT_BUFFER

BSI_UNKNOWN_ERROR 


\section{Discovery Mode:}

\begin{tabular}{|c|c|c|}
\hline \multirow[t]{8}{*}{ Parameters: } & hCard: & Card connection handle from gscBsiutilConnect () \\
\hline & USzAID: & PKI provider module AID value. \\
\hline & unAIDLen : & AID value length in bytes. \\
\hline & ucAlgoID: & $\begin{array}{l}\text { Identifies the cryptographic algorithm that will be used } \\
\text { to generate the signature. All conformant } \\
\text { implementations shall, at a minimum, support } \\
\text { RSA_NO_PAD (Algorithm Identifier 0xA3). } \\
\text { Implementations may optionally support other } \\
\text { algorithms. }\end{array}$ \\
\hline & uszMessage: & The hash of the message to be signed. \\
\hline & unMessageLen: & Length of hashed message to be signed, in bytes. \\
\hline & uszResult: & Set to NULL. \\
\hline & punResultLen: & Length of the required signature buffer in bytes. \\
\hline
\end{tabular}

$\begin{array}{ll}\text { Return Codes: } & \text { BSI_OK } \\ & \text { BSI_BAD_HANDLE } \\ & \text { BSI_SC_LOCKED } \\ & \text { BSI_BAD_AID } \\ & \text { BSI_BAD_PARAM } \\ & \text { BSI_BAD_ALGO_ID } \\ & \text { BSI_CARD_REMOVED } \\ & \text { BSI_ACCESS_DENIED } \\ & \text { BSI_NO_CARDSERVICE } \\ & \text { BSI_UNKNOWN_ERROR }\end{array}$




\section{E.6.4 gscBsiPkiGetCertificate()}

Purpose: $\quad$ Reads the certificate from the card.

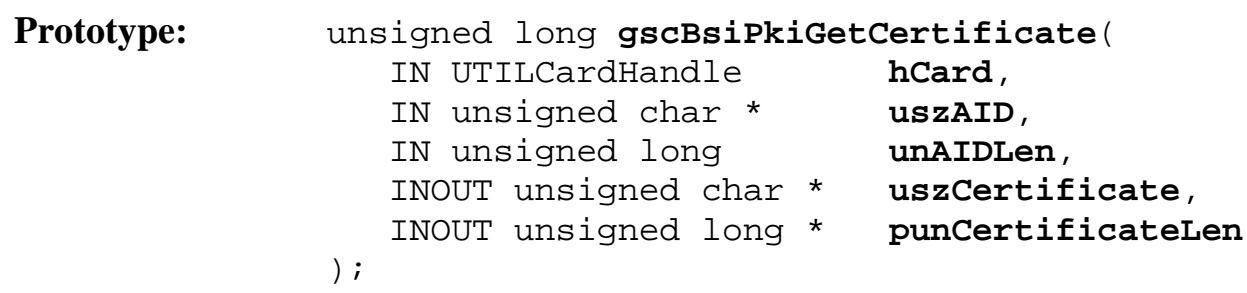

Parameters: hCard:

USzAID :

UnAIDLen:

uszCertificate :

punCertificatelen:
Card connection handle from gscBsiutilconnect () . PKI provider module AID value. The parameter shall be in ASCII hexadecimal format.

AID value length in bytes.

Buffer containing the certificate.

Length of the certificate buffer in bytes.

Return Codes:

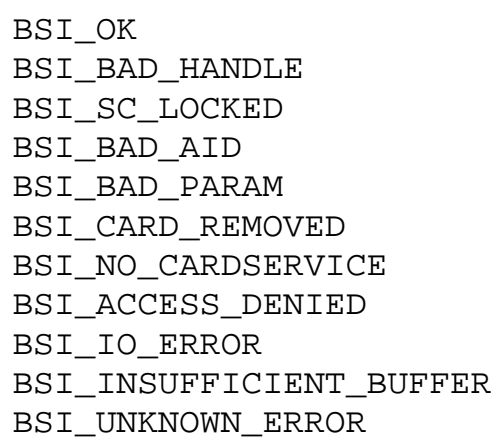

Discovery Mode:
Parameters: hCard:
Card connection handle from gscBsiutilConnect () .
USZAID:
PKI provider module AID value.
UNAIDLen:
AID value length in bytes.
uszCertificate:
Set to NULL.
punCertificatelen: Length of the required certificate buffer in bytes.

$\begin{array}{ll}\text { Return Codes: } & \text { BSI_OK } \\ & \text { BSI_BAD_HANDLE } \\ & \text { BSI_SC_LOCKED } \\ & \text { BSI_BAD_AID } \\ & \text { BSI_BAD_PARAM }\end{array}$


NIST IR 6887 - 2003 EDITION, GSC-IS VERSION 2.1
BSI_CARD_REMOVED
BSI_NO_CARDSERVICE
BSI_ACCESS_DENIED
BSI_IO_ERROR
BSI_UNKNOWN_ERROR 


\section{E.6.5 gscBsiGetCryptoProperties()}

Purpose: $\quad$ Retrieves the Access Control Rules and private cryptographic key length managed by the PKI provider module.

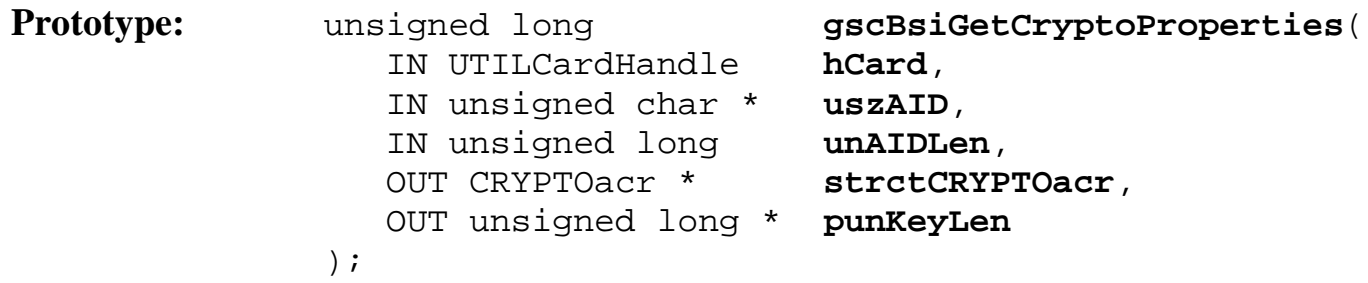

Prototype:

unsigned long

IN UTILCardHandle

IN unsigned char *

IN unsigned long

OUT CRYPTOacr *

OUT unsigned long *

);

\section{Parameters: hCard:}

UszAID :

strctCRYPTOacr:
UnAIDLen:

Card connection handle from gscBsiutilconnect (). AID of the PKI provider. The parameter shall be in ASCII hexadecimal format.

Length of the AID of the PKI provider, in bytes.

Structure indicating access control conditions for all operations. The BSIAcr structure is defined in Section E.5.3. The range of possible values for the members of this structure are defined in Table 3-2 (Section 3.1), and the allowable ACRs for each function in Table 3-4 (Section 3.2). keyIDOrReference contains the key identifier or reference for each access method contained in the ACR in order of appearance. AuthNo is the number of access methods logically combined in the ACR. ACRID is RFU and must be NULL (0x00) in this version. Note that the strctReadValueACR member maps to the gscBsipkiGetCertificate () function.

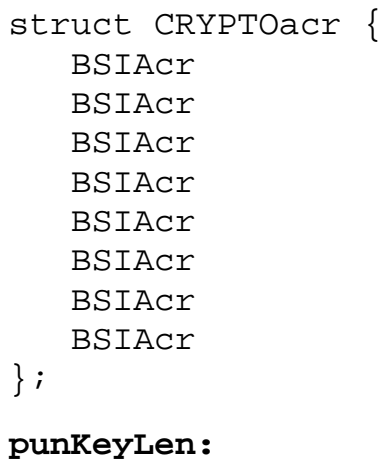

\section{Return Codes:}

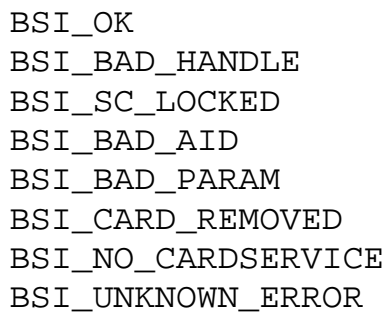

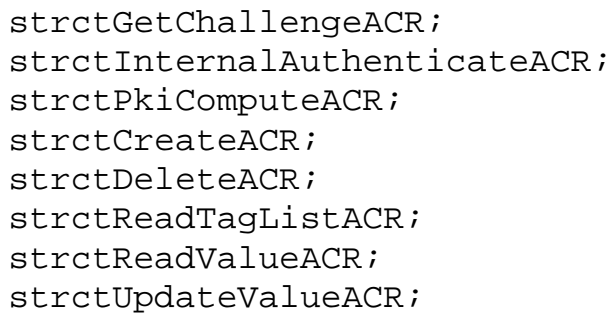

Length of the private key managed by the PKI provider. 
NIST IR 6887 - 2003 EDITION, GSC-IS VERSION 2.1

\section{THIS PAGE INTENTIONALLY LEFT BLANK.}

E-38 


\section{Appendix F-Java Language Binding for BSI Services}

This appendix defines the Java language binding, which comprises a set of classes and interfaces that provide the basic support for a Java implementation of a SPM as defined in the GSC-IS.

Similar to the pseudo IDL specification, the Java translation is logically grouped into three functional modules:

- A Smart Card Utility Provider Module

- A Smart Card Generic Container Provider Module

- A Smart Card Cryptographic Provider Module.

This appendix provides the required syntax and semantics of 23 methods that correspond to the 23 functions in Chapter 4. These methods are collectively defined in the public interface gov.gsc.interfaces.BSI (see Section F.1), and shall be implemented within a public class gov.gsc. classes. GSCBSI. 


\section{F.1 Interfaces and Classes}

Based on the 23 methods described in this appendix (Section F.2 to Section F.4) the interface gov.gsc.interfaces.BSI is defined as follows:

public interface gov.gsc.interfaces.BSI

\{

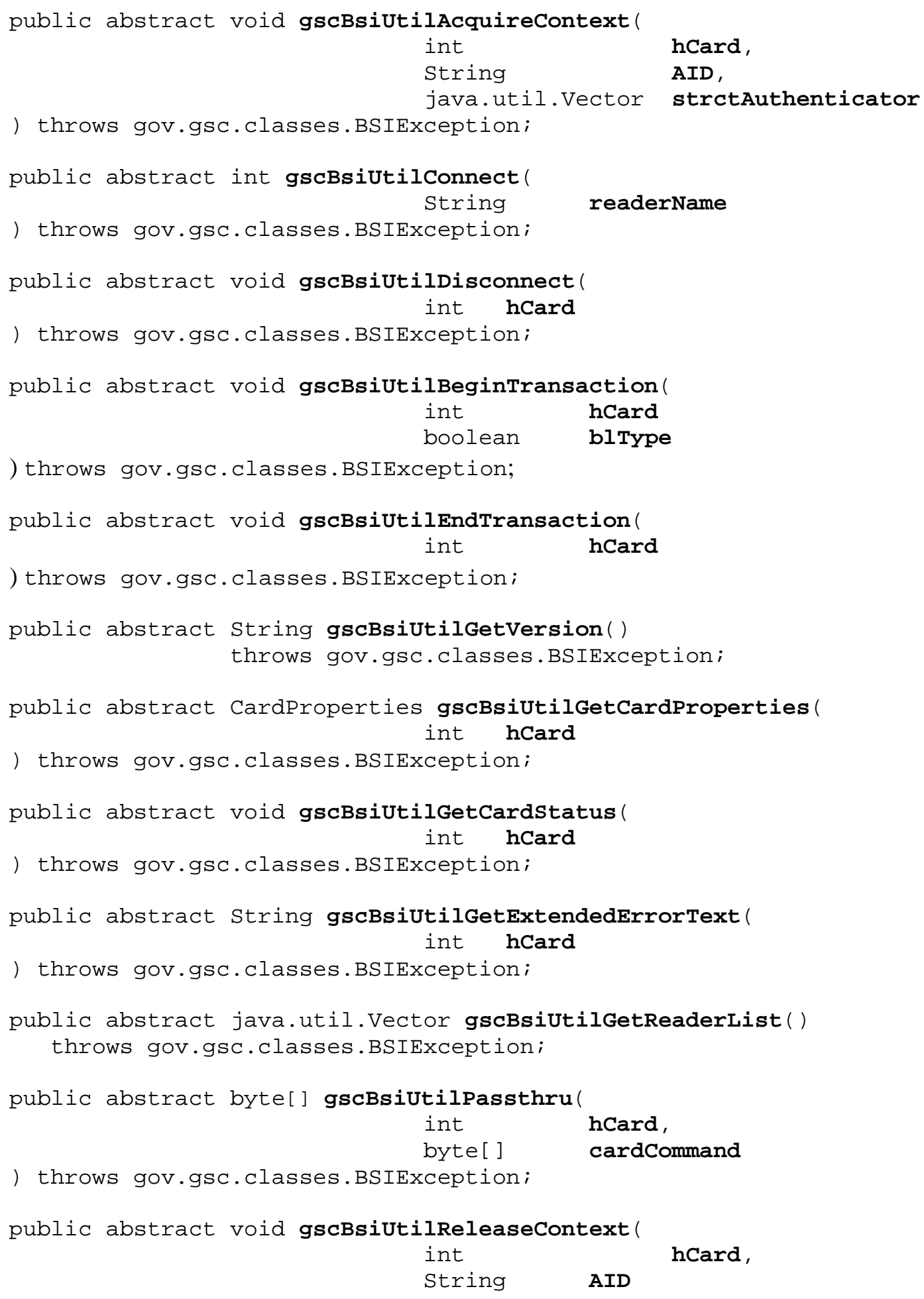




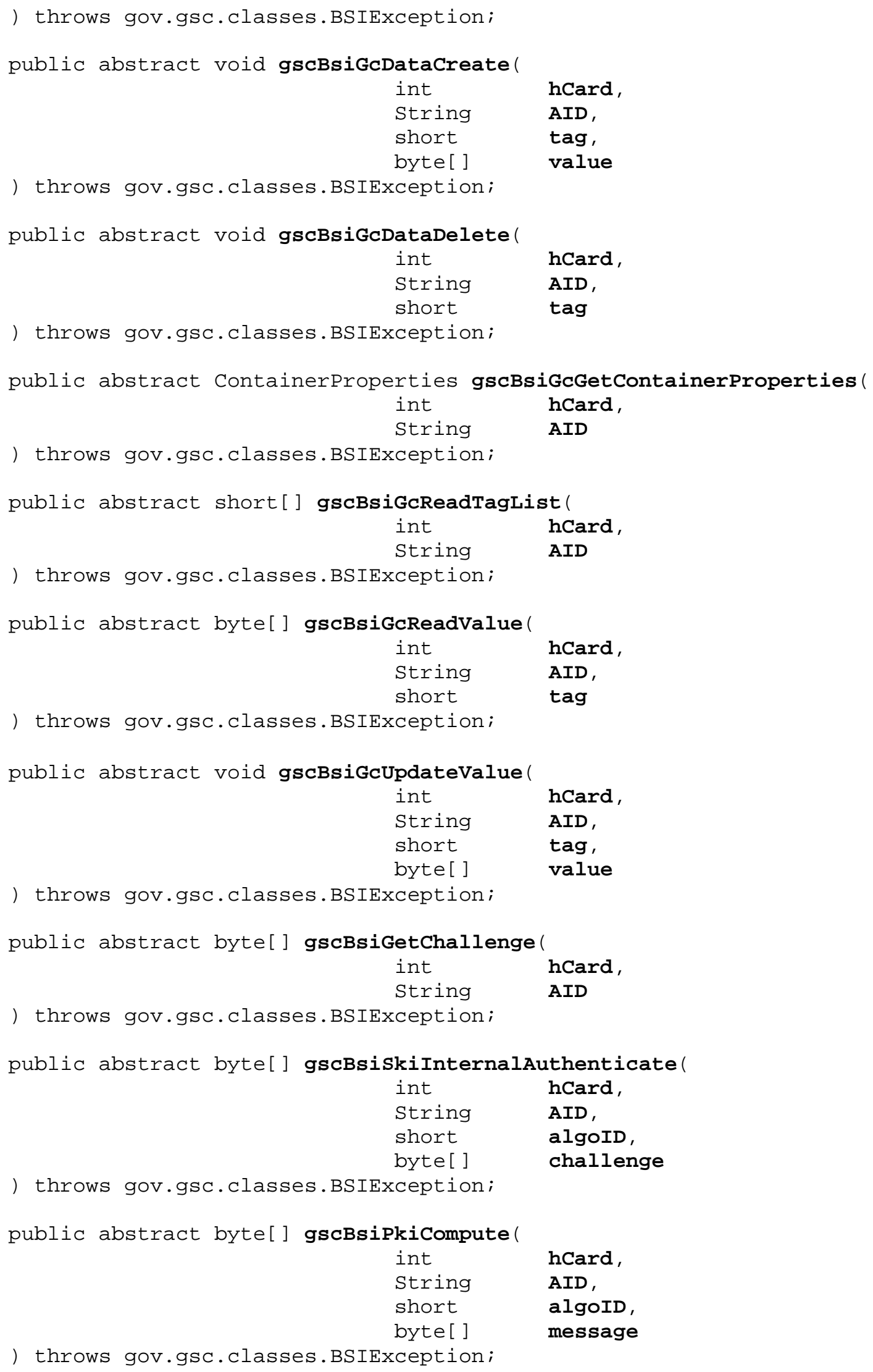




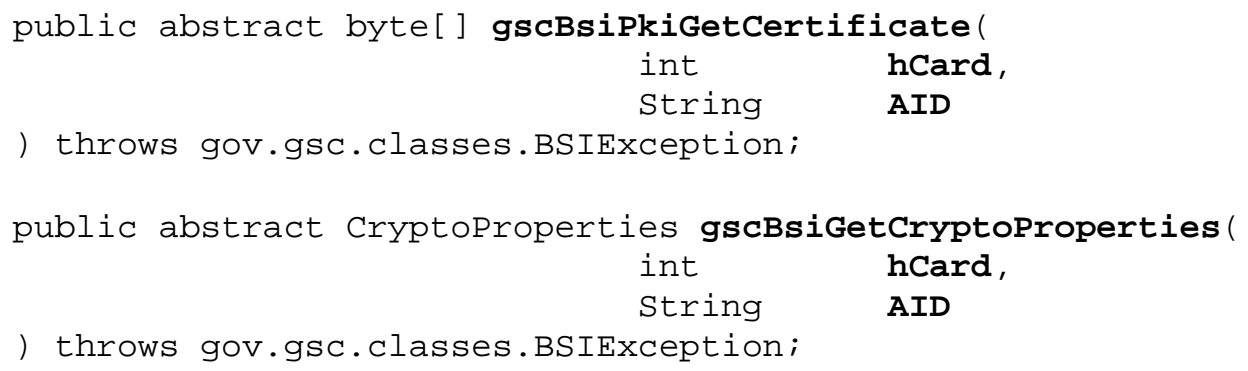

F.1.1 - The Same Class GSCBSI Shall Also Implement The Following Interfaces:
- gov.gsc.interfaces.BSIReturnCodes
- gov.gsc.interfaces.CryptographicAlgoid
- gov.gsc.interfaces.BSIAccesscontrolRules
- gov.gsc.interfaces.BSICardCapabilities

F.1.1.1 - The interfaces are defined as follows:

public interface gov.gsc.interfaces.BSIReturnCodes \{

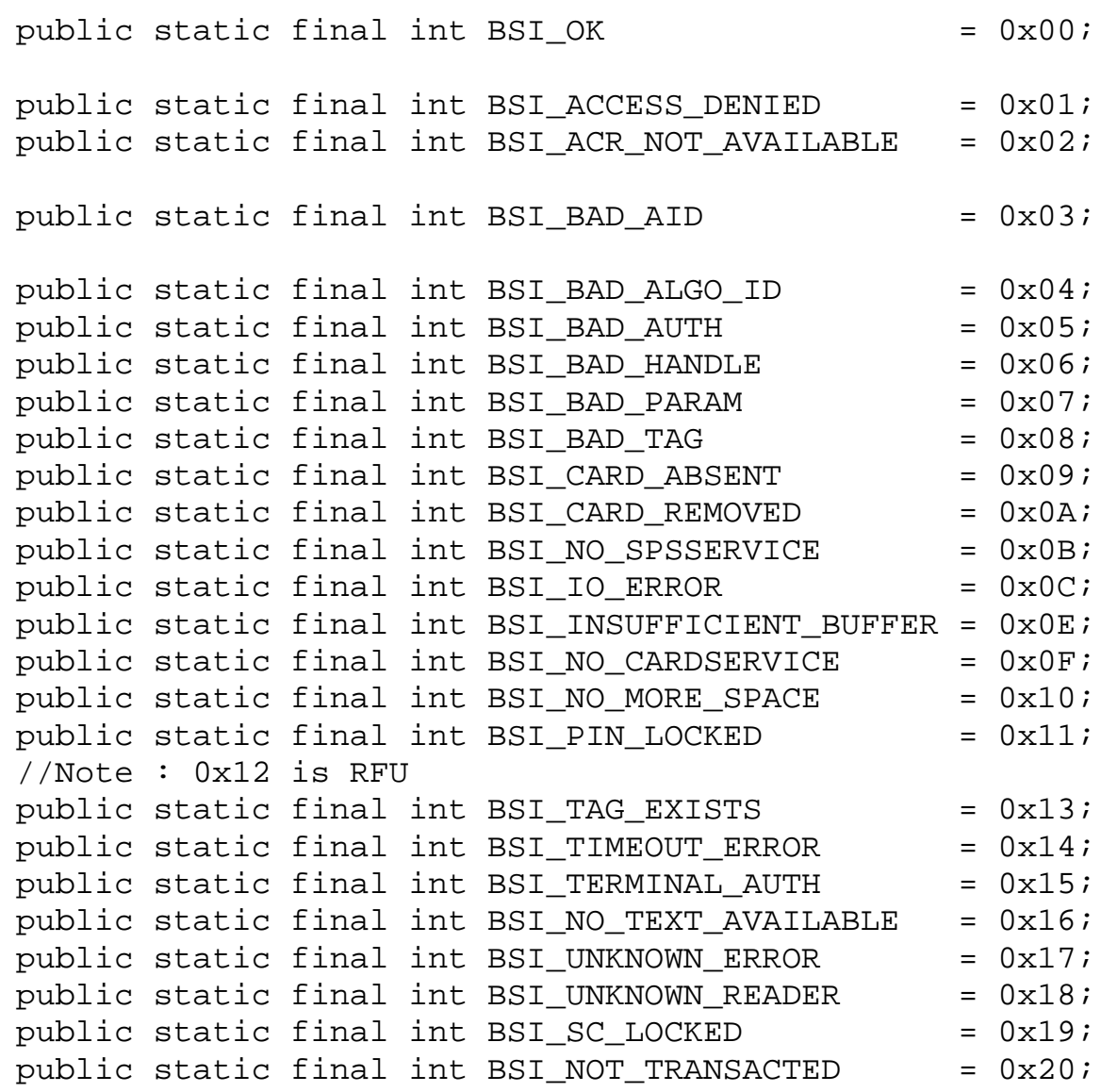


public interface gov.gsc.interfaces.CryptographicAlgoID

\{

//Mandatory Cryptographic Algorithms (see Section 4.3)

//Cryptographic algorithm computation on the private key,

//Chinese Remainder Theory.

public static final short RSA_NO_PAD $=0 \times$ A 3 ;

//DES3-ECB cryptographic algorithm with a double length

//key-size of 16 bytes.

public static final short BSI_DES3_ECB $=0 \times 81$;

//DES3-CBC cryptographic algorithm with a double length

//key-size of 16 bytes .

public static final short BSI_DES3_CBC $=0 \times 82$;

public interface gov.gsc.interfaces.BSIAccessControlRules

\{

//BSI ACR Values as defined in the Table 3-1.

public static final int BSI_ACR_ALWAYS =0x00;

public static final int BSI_ACR_NEVER =0x01;

public static final int BSI_ACR_XAUTH = 0x02;

public static final int BSI_ACR_XAUTH_OR_PIN = 0x03;

public static final int BSI_SECURE_CHANNEL_GP = 0x04;

public static final int BSI_ACR_PIN_ALWAYS = 0x05;

public static final int BSI_ACR_PIN $=0 \times 06$;

public static final int BSI_ACR_XAUTH_THEN_PIN = 0x07;

public static final int BSI_ACR_UPDATE_ONCE = 0x08;

public static final int BSI_ACR_PIN_THEN_XAUTH = 0x09;

//NOTE: 0xOA currently not used

public static final int BSI_SECURE_CHANNEL_ISO $=0 \mathrm{x} 0 \mathrm{~B}$;

public static final int BSI_XAUTH_AND_PIN = OxOC;

//NOTE: RESERVED FOR FUTURE USED OXOD-OXFF

\}

public interface gov.gsc.interfaces.BSICardCapabilities

\{

public static final int BSI_GCCDM =0 0x00000001;

public static final int BSI_SKI $\quad=0 \times 00000002$;

public static final int BSI_PKI $\quad=0 \times 00000004$;

F.1.1.2 - All 23 methods throw a BSIException if an error occurred during execution. A BSIException shall be constructed using one of the eligible return code listed for every individual method.

The class BSIException is defined as follows:

public class gov.gsc.classes.BSIException

extends java.lang.Exception

//Methods inherited from class java.lang.Throwable 


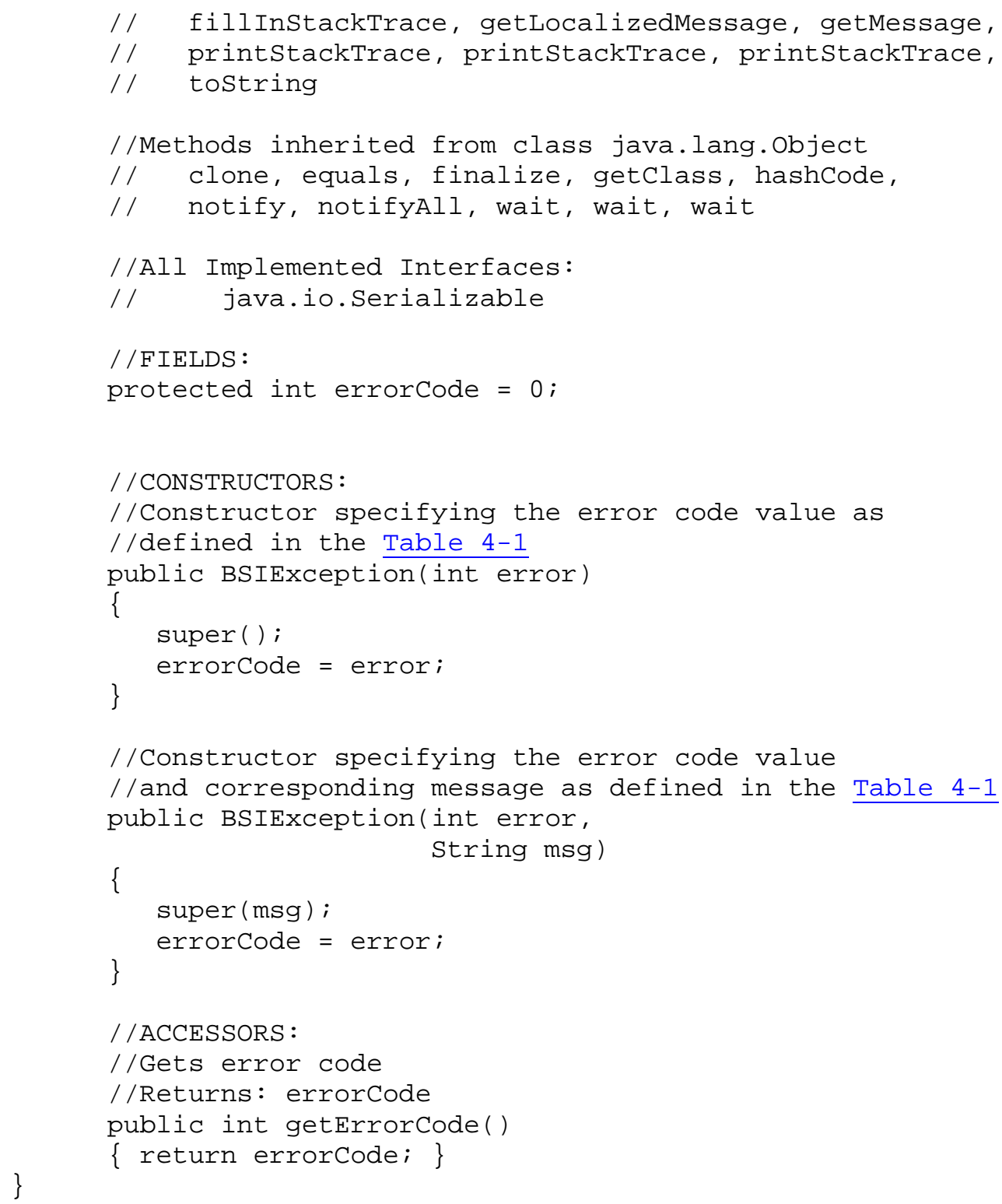




\section{F.2 Smart Card Utility Provider Module Interface Definition}

\section{F.2.1 gscBsiUtilAcquireContext()}

Purpose: $\quad$ This function shall establish a session with a target container on the smart card by submitting the appropriate Authenticator in the BSIAuthenticator structure. For ACRs requiring external authentication (XAUTH), the authValue field of the BSIAuthenticator structure must contain a cryptogram calculated by encrypting a random challenge from gscBsiGetChallenge (). In cases where the card acceptance device authenticates the smart card, this function returns a BSI_TERMINAL_AUTH return code and the cryptogram is ignored.

For ACRs that require chained authentication such as BSI_ACR_PIN_AND_XAUTH, the calling application passes in the required authenticators in multiple BSIAuthenticator structures. In this example the calling application passes a PIN and the appropriate External Authentication cryptogram in two BSIAuthenticator structures. The client application must set the accessMethodType field of each BSIAuthenticator structure to match the type of authenticator contained in the structure. To satisfy an ACR of BSI_ACR_PIN_AND_XAUTH, the application would construct a sequence of two BSIAuthenticators: one containing a PIN and one containing an External Authentication cryptogram. The BSIAuthenticator structure containing the PIN would have an accessMethodType of BSI_AM_PIN, and the BSIAuthenticator structure containing the External Authentication cryptogram would have an accessMethodType of BSI_AM_XAUTH .

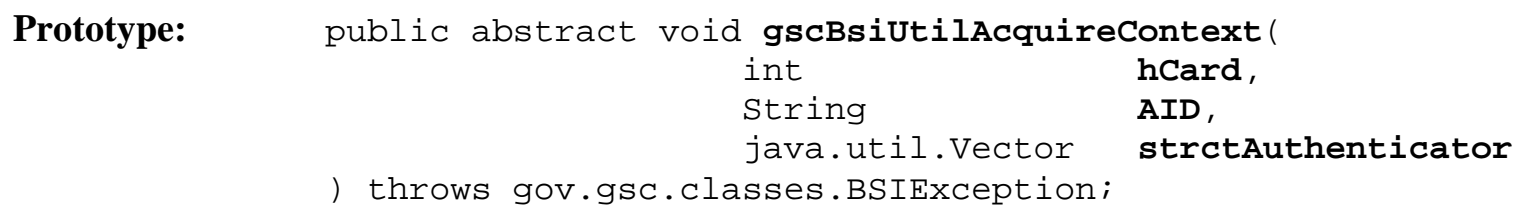

Parameters: hCard: $\quad$ Card connection handle from gscBsiutilconnect ().

AID : AID of the target service provider or container. The AID shall be stored as an ASCII hexadecimal string.

strctAuthenticator: Vector of BSIAuthenticator objects containing the authenticator(s) specified by the ACR required to access a value in the container. The required list of authenticators is returned by gscBsiGcGetContainerProperties(). The calling application is responsible for constructing this object.

The BSIAuthenticator class is defined as follows:

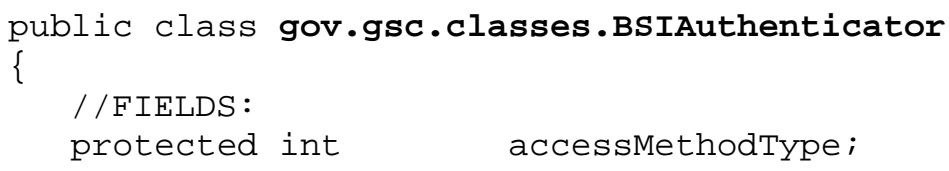




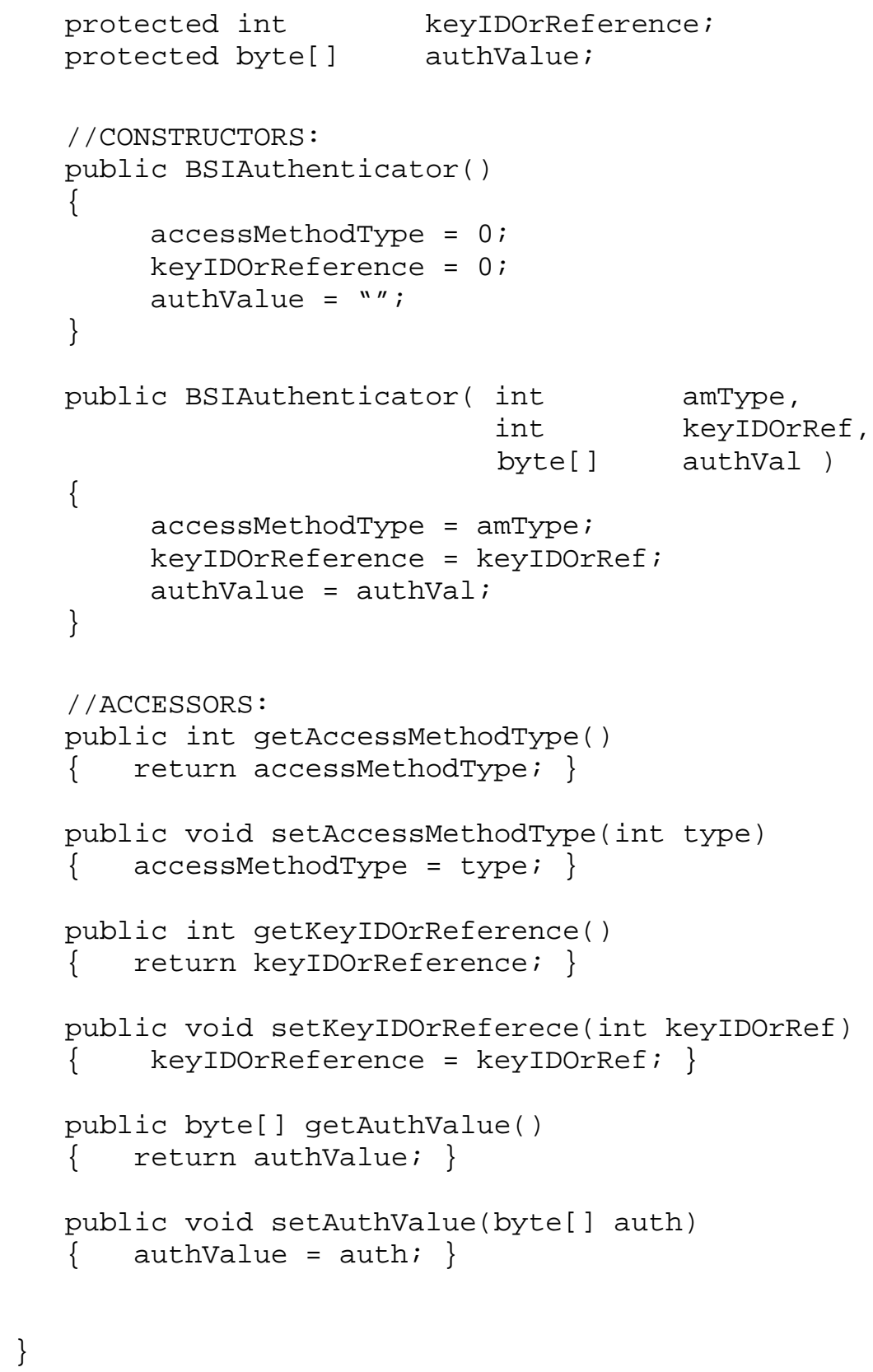

The fields of the BSIAuthenticator class are:

accessmethodType: $\quad$ Access Method Type (see Table 3-1 in Section 3.1).

keyIDOrReference: Key identifier or reference of the authenticator. This is used to distinguish between multiple authenticators with the same Access Method Type.

authValue:

Authenticator, can be an external authentication cryptogram or PIN. If the authenticator value is NULL, then BSI is in charge of gathering authentication information and authenticating to the card. 
NIST IR 6887 - 2003 EDITION, GSC-IS VERSION 2.1

$\begin{array}{ll}\text { Return codes: } & \text { BSI_OK } \\ & \text { BSI_BAD_HANDLE } \\ & \text { BSI_BAD_AID } \\ & \text { BSI_ACR_NOT_AVAILABLE } \\ & \text { BSI_SC_LOCKED } \\ & \text { BSI_BAD_AUTH } \\ & \text { BSI_CARD_REMOVED } \\ & \text { BSI_PIN_BLOCKED } \\ & \text { BSI_TERMINAL_AUTH } \\ & \text { BSI_UNKNOWN_ERROR }\end{array}$




\section{F.2.2 gscBsiUtilConnect()}

Purpose: $\quad$ Establish a logical connection with the card inserted in a specified reader. BSI_TIMEOUT_ERROR will be returned if a connection cannot be established within a specified time. The timeout value is implementation dependent.

Prototype: $\quad \begin{array}{r}\text { public abstract int gscBsiutilConnect( } \\ \text { String readerName }\end{array}$

Parameter: readerName: Name of the reader that the card is inserted into. If this field is an empty String, the SPS shall attempt to connect to the card in the first available reader, as returned by a call to the BSI's function gscBsiutilGetReaderList (). The Name of the reader shall be stored as ASCII encoding Strings. (See Section 4.2)

Return Value: hcard: Card connection handle.

Return codes: $\quad$ BSI_OK

BSI_UNKNOWN_READER

BSI_CARD_ABSENT

BSI_TIMEOUT_ERROR

BSI_UNKNOWN_ERROR 


\section{F.2.3 gscBsiUtilDisconnect()}

Purpose: $\quad$ Terminate a logical connection to a card.

Prototype: public abstract void gscBsiutildisconnect (

) throws gov.gsc.classes.BSIException;

Parameter: hCard: Card connection handle from gscBsiutilconnect () .

Return codes: $\quad$ BSI_OK

BSI_BAD_HANDLE

BSI_CARD_REMOVED

BSI_UNKNOWN_ERROR 


\section{F.2.4 gscBsiUtilBeginTransaction()}

Purpose: $\quad$ Starts an exclusive transaction with the smart card referenced by hCard. When the transaction starts, all other applications are precluded from accessing the smart card while the transaction is in progress. Two types of calls can be made with that function: a blocking transaction call and a non-blocking transaction call. A boolean type parameter identify which mode is called. In the non-blocking mode, the call will return immediately if another client has an active transaction lock. The returned error code will be BSI_SC_LOCKED. In the blocking mode, the call will wait indefinitely for any active transaction locks to be released. A transaction must be completed by a call to gscBsiutilendTransaction() .

For single-threaded BSI implementations, it can be assumed that each application will be associated with a separate process. The same process that starts a transaction must also complete the transaction. For multi-threaded BSI implements, it can be assumed that each application will be associated with a separate thread and/or process. The same thread that starts a transaction must also complete the transaction.

If this function is called by a thread that has already called gscBsiutilBeginTransaction() but has not yet called gscBsiUtilendTransaction() it will return the error BSI_NOT_TRANSACTED.

If the SPS (Service Provider Software) does not support transaction locking, it should return the error code BSI_NO_SPSSERVICE in response to a call to

gscBsiutilBeginTransaction().

Prototype:

public abstract void gscBsiutilBeginTransaction(

int hCard

boolean bltype

) throws gov.gsc.classes.BSIException;

Parameters: hCard: $\quad$ Card communication handle returned from gscBsiutilConnect ()

blType:

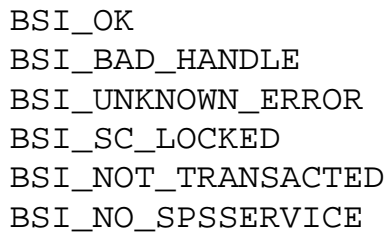

Boolean specifying the type of transaction call ( blType set to "true" in blocking mode. blType set to "false" in non blocking mode). 


\section{F.2.5 gscBsiUtilEndTransaction()}

Purpose: Completes a previously started transaction, allowing other applications to resume interactions with the card.

If this function is called by a thread that has not yet called gscBsiutilBeginTransaction() it will return the error BSI_NOT_TRANSACTED.

If the SPS (Service Provider Software) does not support transaction locking, it should return the error code BSI_NO_SPSSERVICE in response to a call to gscBsiutilendTransaction().

Prototype: public abstract voidgscBsiutilendTransaction( int hCard

) throws gov.gsc.classes.BSIException;

Parameters: hCard: Card communication handle returned from gscBsiutilConnect ().

Return Codes: $\quad$ BSI_OK

BSI_BAD_HANDLE

BSI_UNKNOWN_ERROR

BSI_NOT_TRANSACTED

BSI_NO_SPSSERVICE 


\section{F.2.6 gscBsiUtilGetVersion()}

Purpose: $\quad$ Returns the BSI implementation version.

Prototype: $\quad$ public abstract String gscBsiutilgetVersion(

) throws gov.gsc.classes.BSIException;

Return Value: version: $\quad$ A String representing the BSI and SPS's version formatted as "major,minor,revision,build_number". The value for an SPS conformant with this version of the GSC-IS is " $2,1,0,<$ build number >". The build number field is vendor/implementation dependent. The version shall be stored as ASCII encoded Strings. (See Section 4.2)

Return codes: $\quad$ BSI_OK

BSI_UNKNOWN_ERROR 


\section{F.2.7 gscBsiUtilGetCardProperties()}

Purpose: $\quad$ Retrieves ID and capability information for the card.

Prototype: public abstract CardProperties gscBsiutilGetCardProperties ( int hCard

) throws gov.gsc.classes.BSIException;

Parameter: hCard: $\quad$ Card connection handle from gscBsiutilconnect ().

Return Value: cardProps : A CardProperties object defined as follows:

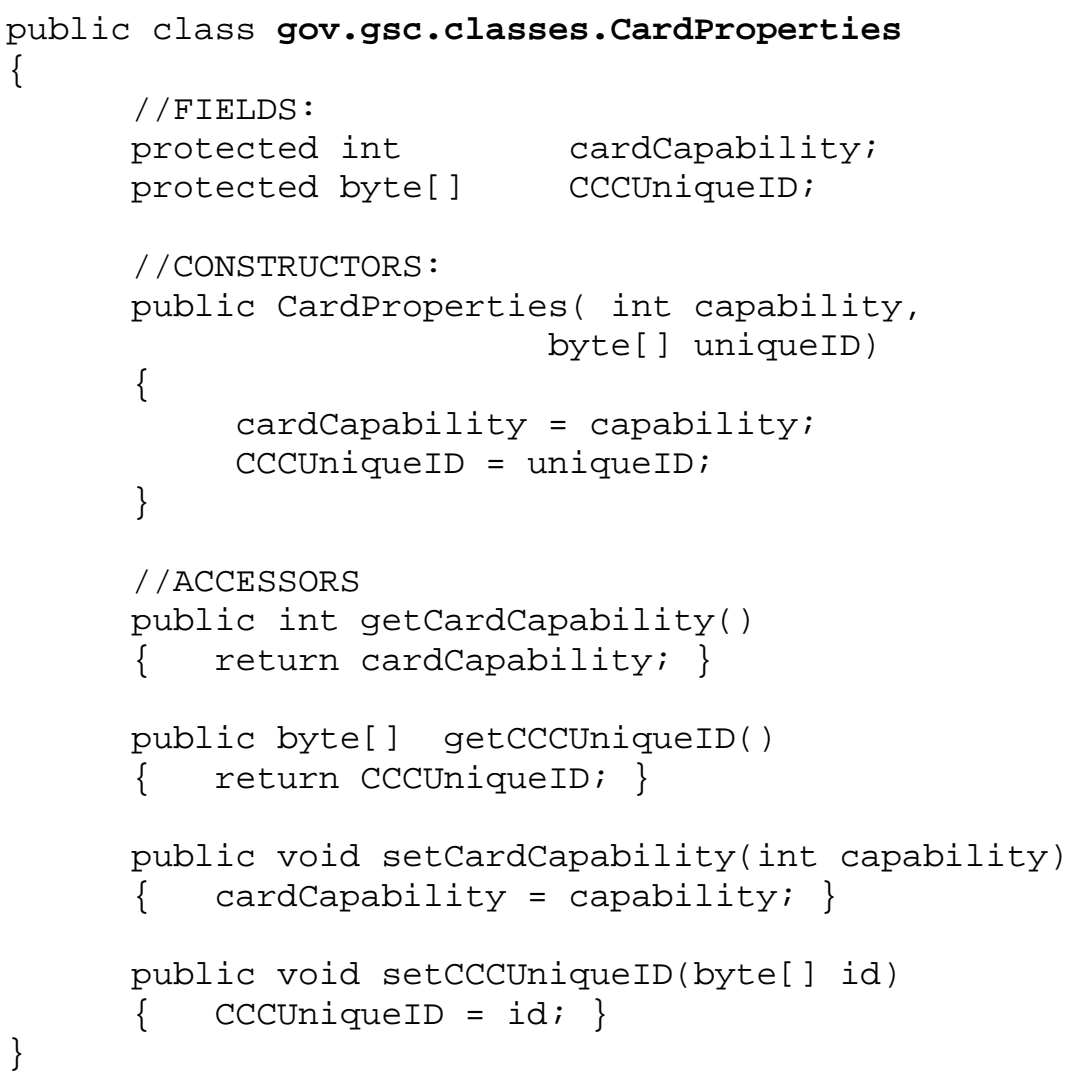

where the fields are described as follows:

CCCUniqueID: $\quad$ String for the Card Capability Container ID.

cardCapability: $\quad$ Bit mask value defining the providers supported by the card. The bit masks represent the Generic Container Data Model, the Generic Container Data Model Extended, the Symmetric Key Interface, and the Public Key Interface providers respectively.

\footnotetext{
Return codes: $\quad$ BSI_OK

BSI_BAD_HANDLE

BSI_CARD_REMOVED

BSI_SC_LOCKED
} 
NIST IR 6887 - 2003 EDITION, GSC-IS VERSION 2.1

BSI_NO_CARDSERVICE

BSI_UNKNOWN_ERROR 


\section{F.2.8 gscBsiUtilGetCardStatus()}

Purpose: $\quad$ Checks whether a given card's handle is associated with a card that is inserted into a powered up reader.

Prototype: public abstract void gscBsiutilgetCardstatus (

$$
\text { int hCard }
$$

) throws gov.gsc.classes.BSIException;

Parameters: hCard: $\quad$ Card connection handle from gscBsiutilconnect ().

Return codes: $\quad$ BSI_OK

BSI_BAD_HANDLE

BSI_CARD_REMOVED

BSI_UNKNOWN_ERROR 


\section{F.2.9 gscBsiUtilGetExtendedErrorText()}

Purpose: $\quad$ When a BSI function call throws a BSIException, an application can make a subsequent call to this function to receive additional error information from the card reader driver layer, if available. Since the GSC-IS architecture accommodates different card reader driver layers, the error text information will be dependent on the card reader driver layer used in a particular implementation. This function must be called immediately after the error has occurred.

Prototype: $\quad$ public abstract String gscBsiutilGetExtendedErrorText (
) throws gov.gsc.classes.BSIException;

Parameters: hCard: $\quad$ Card connection handle gscBsiutilConnect () .

Return Value: errorText : A String of maximum 255 characters including the null terminator, containing an implementation specific error text. If an extended error text string is not available, this function returns an empty string and BSI_NO_TEXT_AVAILABLE. The error text shall be stored as ASCII encoding Strings. (See Section 4.2)

Return Codes: $\quad$ BSI_OK

BSI_BAD_HANDLE

BSI_NO_TEXT_AVAILABLE

BSI_UNKNOWN_ERROR 


\section{F.2.10 gscBsiUtilGetReaderList()}

Purpose: $\quad$ Retrieves the list of available readers.

Prototype: public abstract java.util.Vector gscBsiutilGetReaderList() throws gov.gsc.classes.BSIException;

Return Value: vReaderList: Vector of Strings containing a list of the available readers. The Strings shall be in ASCII format.

Return codes: $\quad$ BSI_OK

BSI_UNKNOWN_ERROR 


\section{F.2.11 gscBsiUtilPassthru()}

Purpose: $\quad$ Allows a client application to send a "raw" APDU through the BSI directly to the card and receive the APDU-level response.

Prototype: public abstract byte[] gscBsiutilPassthru

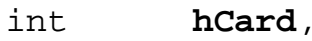

byte [] cardCommand

) throws gov.gsc.classes.BSIException;

Parameters: hCard: $\quad$ Card connection handle from gscBsiutilConnect ().

cardCommand:

Return Value:

\section{Return codes:}

cardResponse :

BSI_OK
BSI_BAD_HANDLE
BSI_BAD_PARAM
BSI_SC_LOCKED
BSI_CARD_REMOVED
BSI_UNKNOWN_ERROR

An array of bytes representing the APDU response from the card. The parameter must be in ASCII hexadecimal format. The response must include the status bytes SW1 and SW2 returned by the card. 


\section{F.2.12 gscBsiUtilReleaseContext()}

Purpose: $\quad$ Terminate a session with the target container on the card.

Prototype: public abstract void gscBsiutilReleasecontext(
int
String AID
) throws gov.gsc.classes.BSIException;

Parameters: hCard: $\quad$ Card connection handle from gscBsiutilconnect ().

AID: $\quad$ Target container AID value. The AID shall be stored as an ASCII hexadecimal string.

$\begin{array}{ll}\text { Return codes: } & \text { BSI_OK } \\ & \text { BSI_BAD_HANDLE } \\ & \text { BSI_BAD_AID } \\ & \text { BSI_SC_LOCKED } \\ & \text { BSI_CARD_REMOVED } \\ & \text { BSI_UNKNOWN_ERROR }\end{array}$




\section{F.3 Smart Card Generic Container Provider Module Interface Definition}

\section{F.3.1 gscBsiGcDataCreate()}

Purpose: $\quad$ Create a new data item in $\{$ Tag, Length, Value $\}$ format in the selected container.

Prototype: public abstract void gscBsiGcDataCreate (
int
String AID,
short tag,
byte[] value
) throws gov.gsc.classes.BSIException;

Parameters: hCard: $\quad$ Card connection handle from gscBsiutilconnect ().

AID: $\quad$ Target container AID value. The AID shall be stored as an ASCII hexadecimal string.

tag: $\quad$ Tag of data item to store.

value: $\quad$ Data value to store.

$\begin{array}{ll}\text { Return codes: } & \text { BSI_OK } \\ & \text { BSI_BAD_HANDLE } \\ & \text { BSI_BAD_AID } \\ & \text { BSI_BAD_PARAM } \\ & \text { BSI_SC_LOCKED } \\ & \text { BSI_CARD_REMOVED } \\ & \text { BSI_NO_CARDSERVICE } \\ & \text { BSI_ACCESS_DENIED } \\ & \text { BSI_NO_MORE_SPACE } \\ & \text { BSI_TAG_EXISTS } \\ & \text { BSI_IOERROR } \\ & \text { BSI_UNKNOWN_ERROR }\end{array}$




\section{F.3.2 gscBsiGcDataDelete()}

Purpose: $\quad$ Delete the data item associated with the tag value in the specified container.

Prototype: $\quad$ public abstract void gscBsiGcDataDelete (
int
String AID,
short tag
) throws gov.gsc.classes.BSIException;

Parameters: hCard: Card connection handle from gscBsiutilconnect ().

AID: $\quad$ Target container AID value. The AID shall be stored as an ASCII hexadecimal string.

tag: $\quad$ Tag of data item to delete.

$\begin{array}{ll}\text { Return codes: } & \text { BSI_OK } \\ & \text { BSI_BAD_HANDLE } \\ & \text { BSI_BAD_AID } \\ & \text { BSI_BAD_TAG } \\ & \text { BSI_SC_LOCKED } \\ & \text { BSI_CARD_REMOVED } \\ & \text { BSI_NO_CARDSERVICE } \\ & \text { BSI_ACCESS_DENIED } \\ & \text { BSI_IO_ERROR } \\ & \text { BSI_UNKNOWN_ERROR }\end{array}$




\section{F.3.3 gscBsiGcGetContainerProperties()}

Purpose: $\quad$ Retrieves the properties of the specified container.

Prototype:
gublic abstract ContainerProperties
gscBscGetContainerProperties(
int heard,
String AID
) throws gov.gsc.classes.BSIException;

Parameters: hCard: $\quad$ Card connection handle from gscBsiUtilConnect () .

AID: $\quad$ Target container AID value. The AID shall be stored as an ASCII hexadecimal string.

Return Value: containerProps: A ContainerProperties object defined as follows:

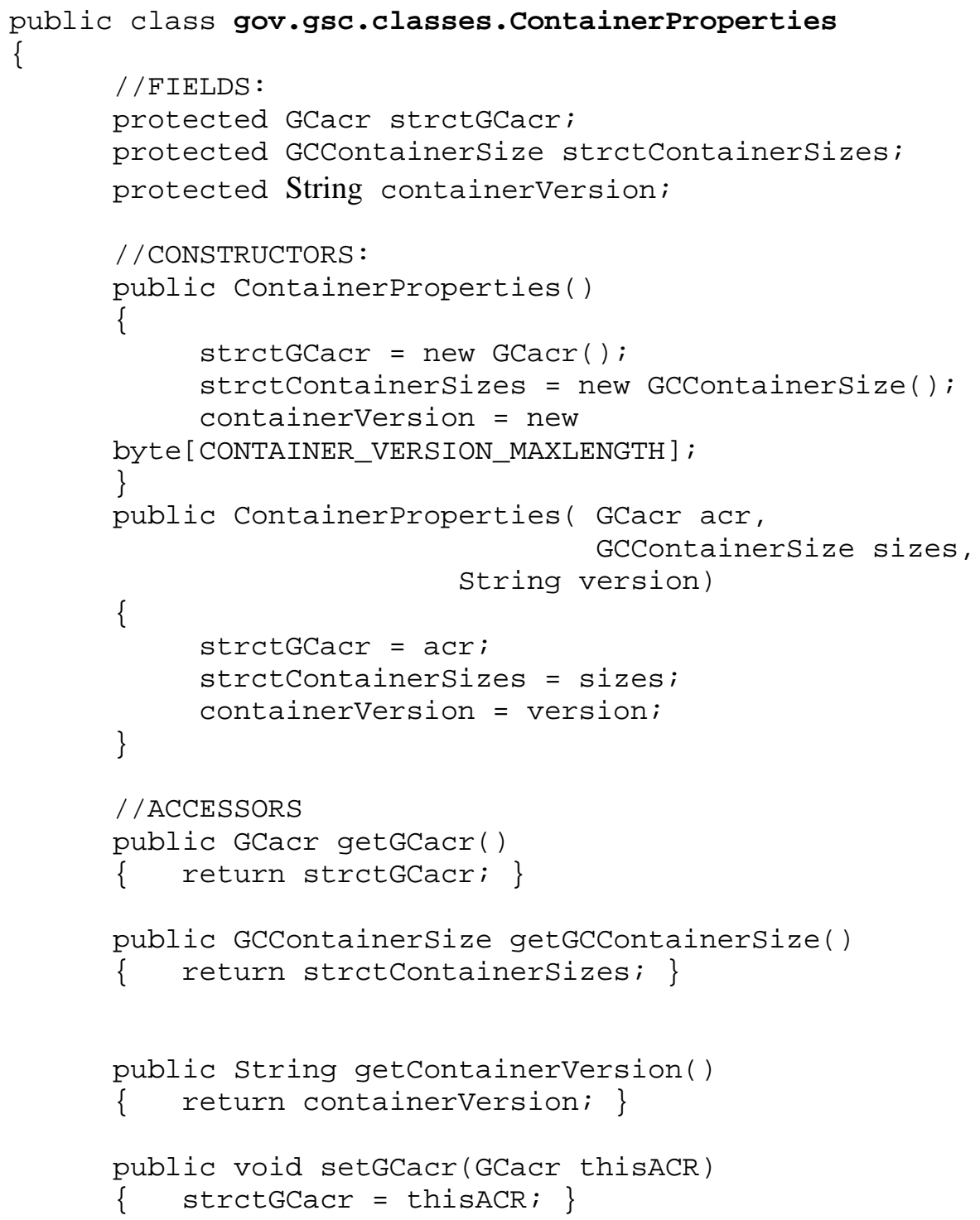




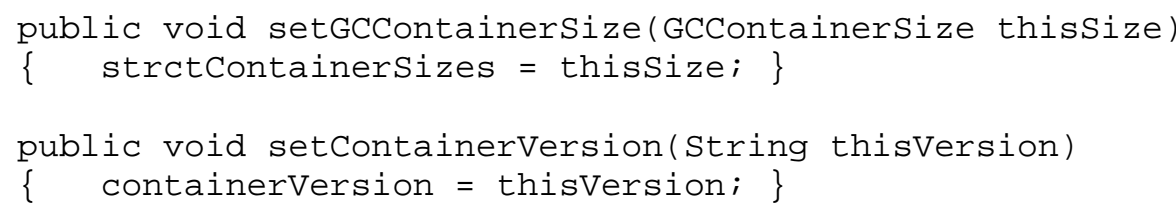

where the fields are described as follows:

strctGCacr:

Object indicating access control conditions for all operations. The range of possible values for the instance variables of this object is defined in Table 3-2 (Section 3.1). The allowable ACRs for each function are listed in Table 3-3 (Section 3.2). key IDOrReference contains the key identifier or reference for each access method contained in the ACR in order of appearance. AuthNb is the number of access methods logically combined in the ACR. ACRID is RFU and must be NULL (0x00) in this version.

The class GCacr is defined as follows:

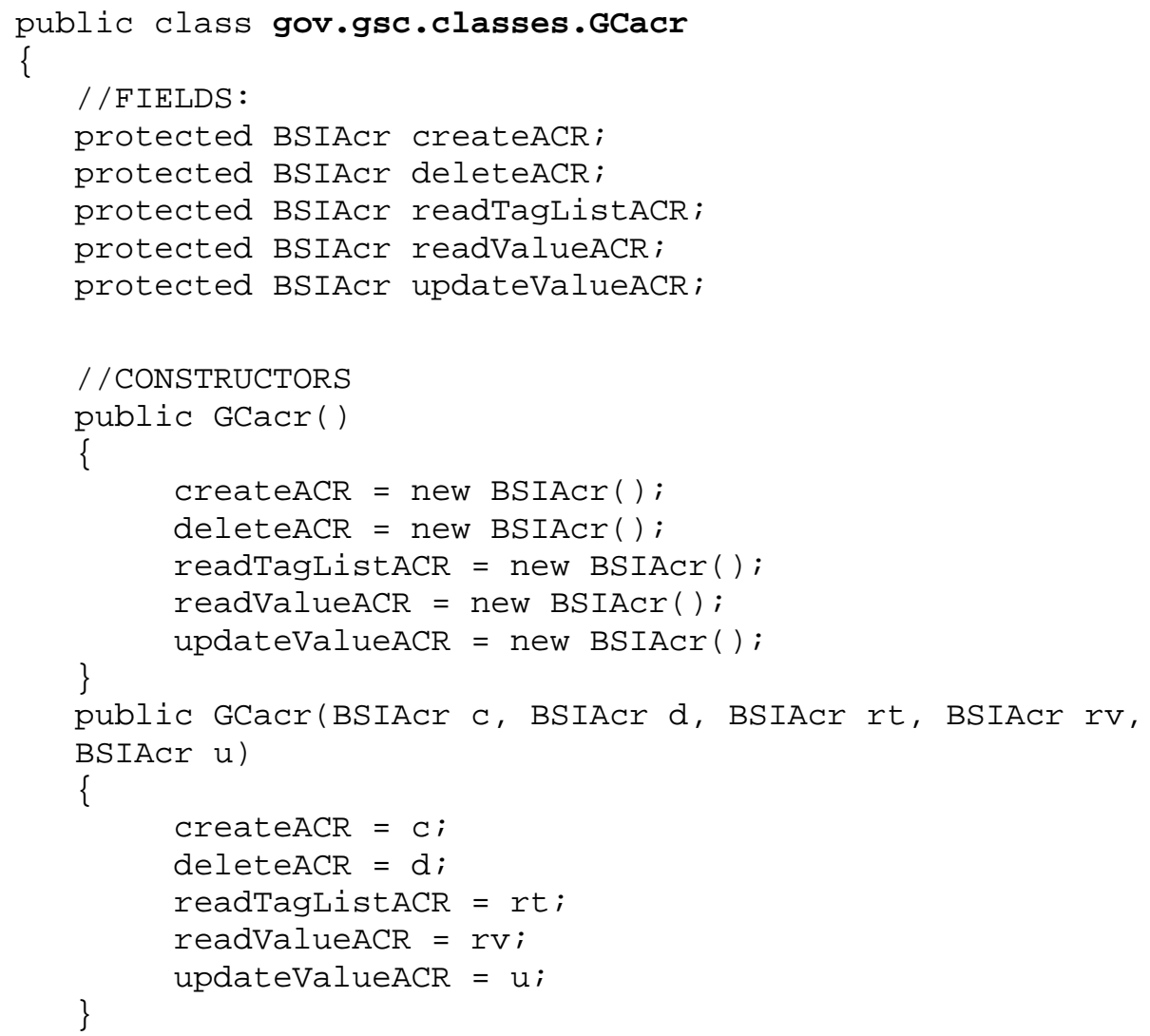




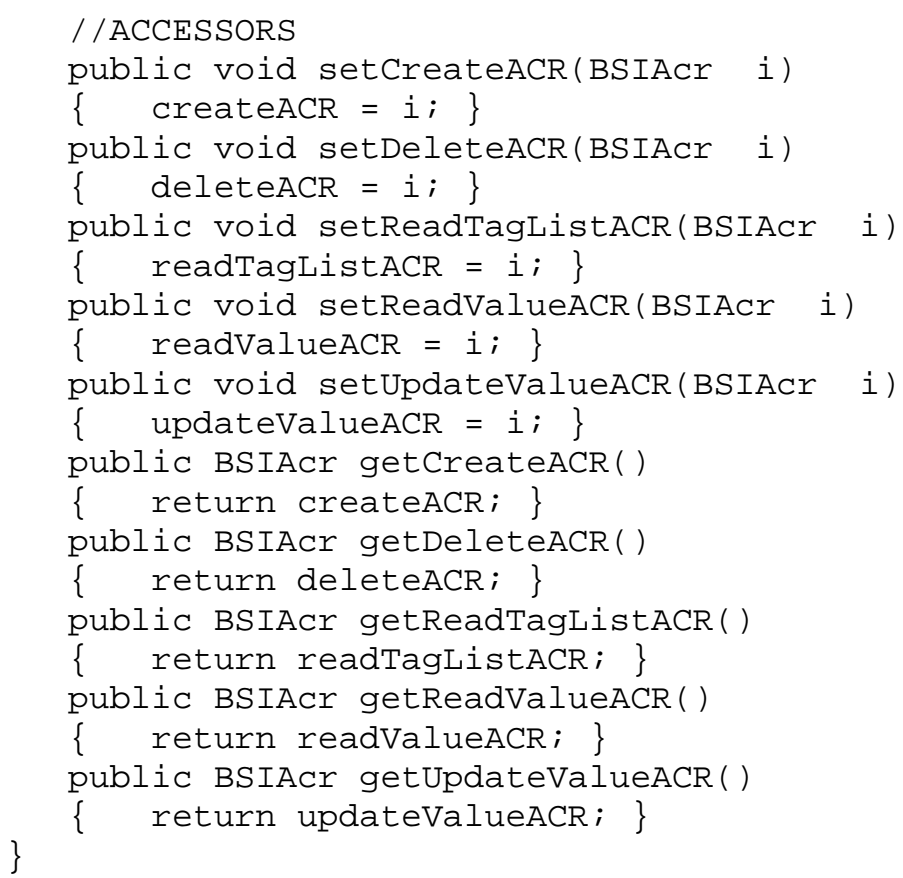

The class BSIAcr is defined as follows:

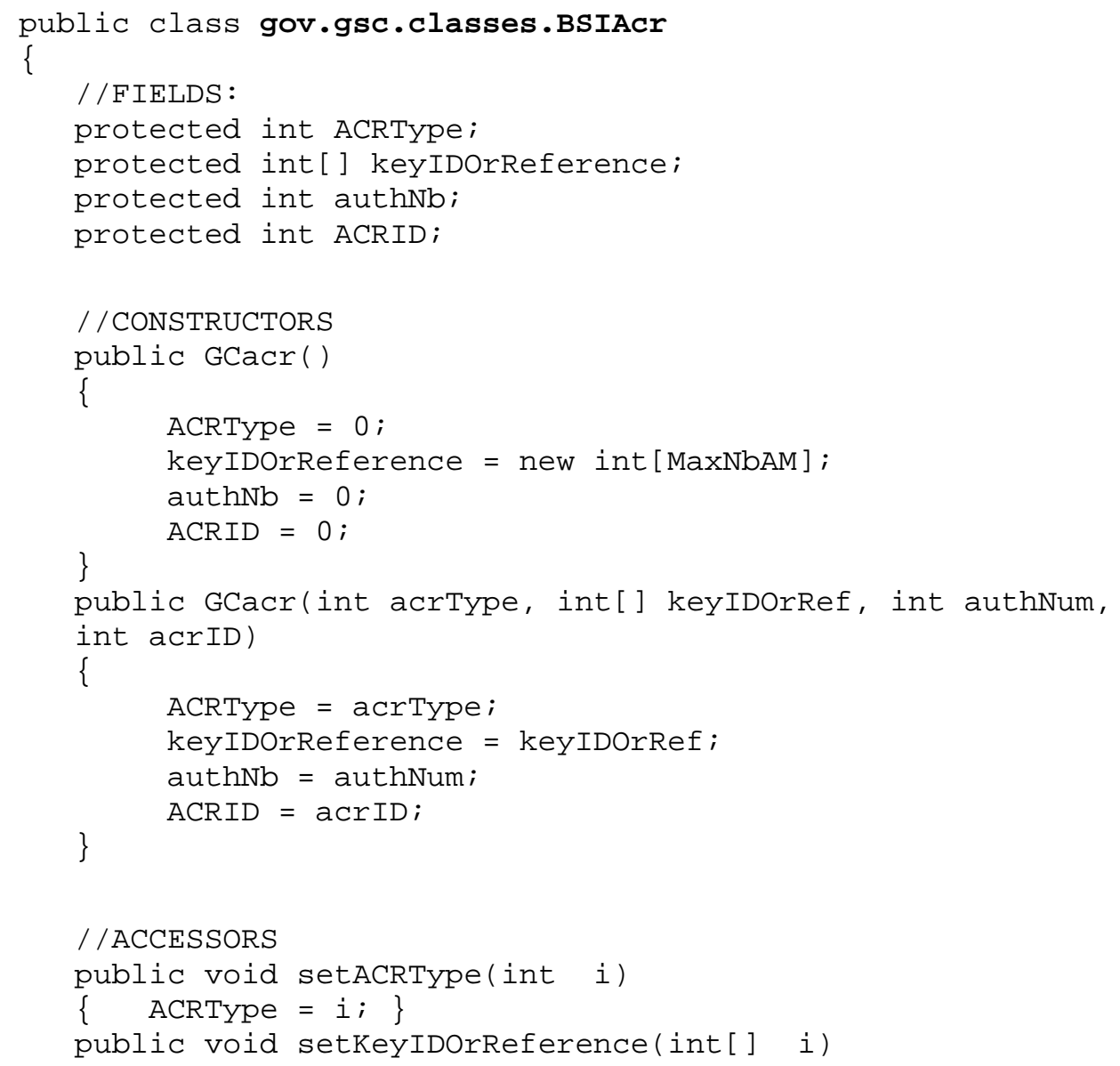




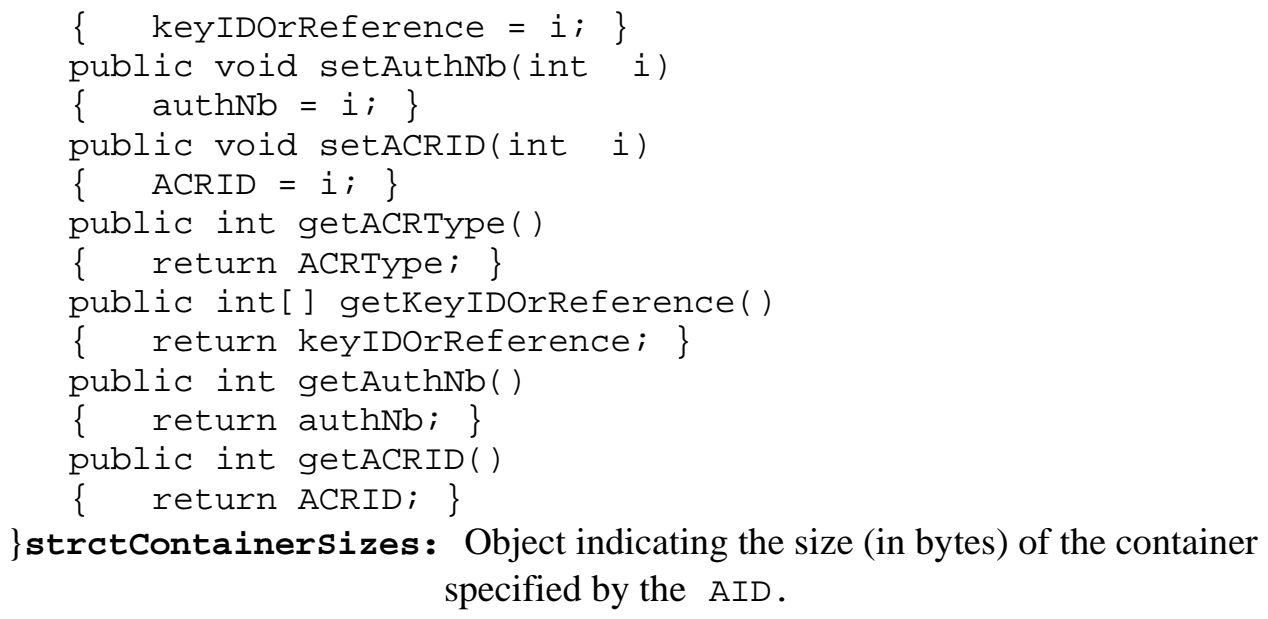
specified by the AID.

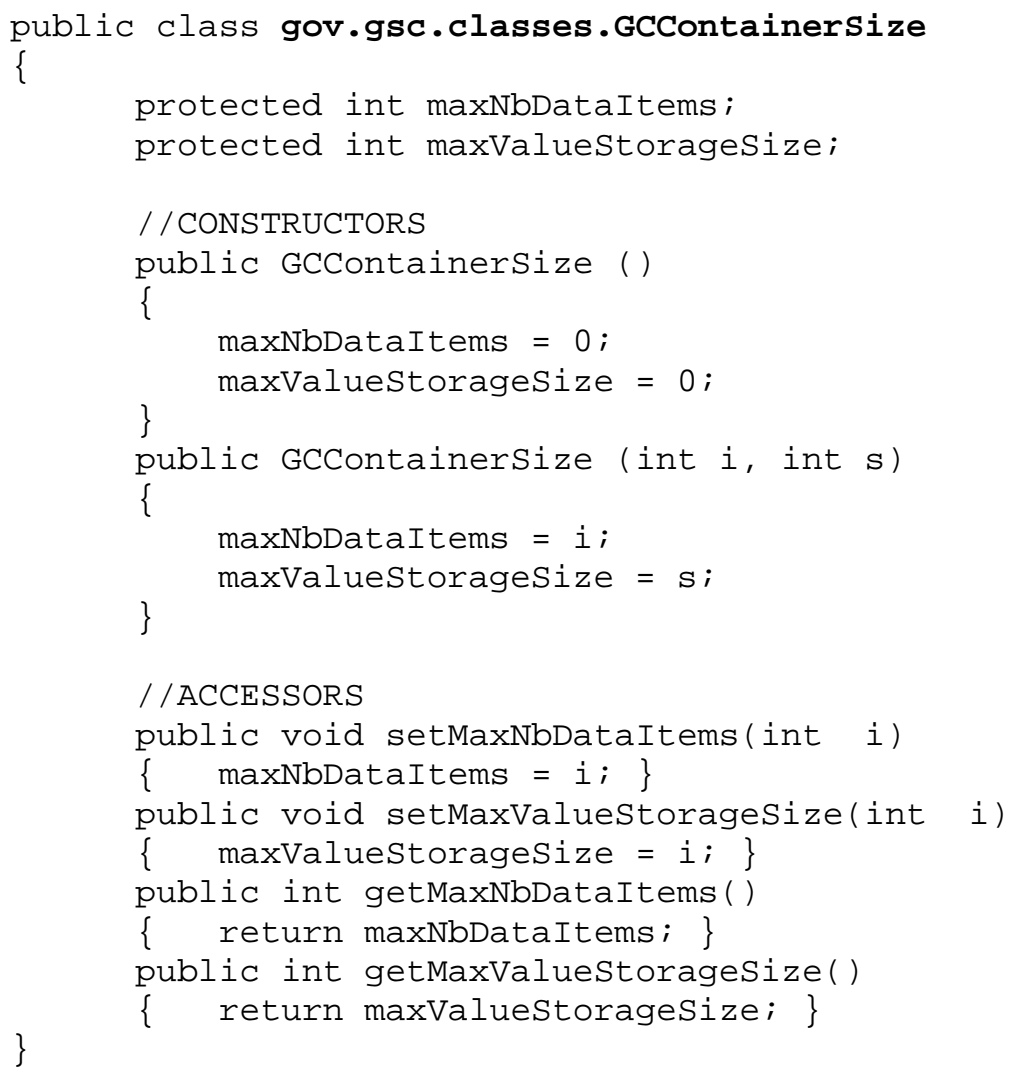

\section{Return codes: $\quad$ BSI_OK}

BSI_BAD_HANDLE

BSI_BAD_AID

BSI_SC_LOCKED

BSI_CARD_REMOVED

BSI_NO_CARDSERVICE

BSI_UNKNOWN_ERROR 


\section{F.3.4 gscBsiGcReadTagList()}

Purpose: $\quad$ Return the list of tags in the selected container.

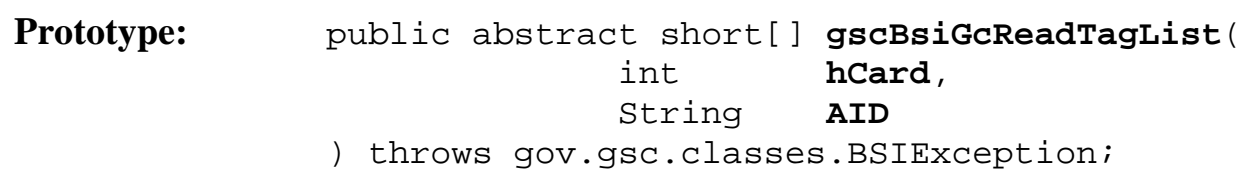

Parameters: hCard: Card connection handle from gscBsiutilconnect () .

AID: $\quad$ Target container AID value. The AID shall be stored as an ASCII hexadecimal string.

Return Value: tagListarray: An array containing the list of tags for the selected container. The tags shall be of the type "short".

$\begin{array}{ll}\text { Return codes: } & \text { BSI_OK } \\ & \text { BSI_BAD_HANDLE } \\ & \text { BSI_SC_LOCKED } \\ & \text { BSI_BAD_AID } \\ & \text { BSI_CARD_REMOVED } \\ & \text { BSI_NO_CARDSERVICE } \\ & \text { BSI_ACCESS_DENIED } \\ \text { BSI_UNKNOWN_ERROR }\end{array}$




\section{F.3.5 gscBsiGcReadValue()}

Purpose: $\quad$ Returns the Value associated with the specified Tag.

\begin{tabular}{|c|c|c|}
\hline Prototype: & $\begin{array}{r}\text { public abstract byte [] } \\
\text { int } \\
\text { String } \\
\text { short }\end{array}$ & $\begin{array}{l}\text { gscBsigcReadValue } \\
\text { hCard, } \\
\text { AID, } \\
\text { tag }\end{array}$ \\
\hline
\end{tabular}

Parameters: hCard: Card connection handle from gscBsiutilconnect () .

AID: $\quad$ Target container AID value. The AID shall be stored as an ASCII hexadecimal string.

tag: Tag value of data item to read.

Return Value: value: Data Value associated with the specified tag.

$\begin{array}{ll}\text { Return codes: } & \text { BSI_OK } \\ & \text { BSI_BAD_HANDLE } \\ & \text { BSI_SC_LOCKED } \\ & \text { BSI_BAD_AID } \\ & \text { BSI_BAD_TAG } \\ & \text { BSI_CARD_REMOVED } \\ & \text { BSI_NO_CARDSERVICE } \\ & \text { BSI_ACCESS_DENIED } \\ & \text { BSI_IO_ERROR } \\ & \text { BSI_UNKNOWN_ERROR }\end{array}$




\section{F.3.6 gscBsiGcUpdateValue()}

Purpose: $\quad$ Updates the Value associated with the specified Tag.

Prototype: public abstract void gscBsiGcUpdatevalue (
int
String AID,
short tag,
byte[] value
) throws gov.gsc.classes.BSIException;

Parameters: hCard: $\quad$ Card connection handle from gscBsiutilconnect () .

AID: $\quad$ Target container AID value. The AID shall be stored as an ASCII hexadecimal string.

tag: $\quad$ Tag of data item to update.

value: $\quad$ New Value of the data item.

$\begin{array}{ll}\text { Return codes: } & \text { BSI_OK } \\ & \text { BSI_BAD_HANDLE } \\ & \text { BSI_BAD_AID } \\ & \text { BSI_SC_LOCKED } \\ & \text { BSI_BAD_PARAM } \\ & \text { BSI_BAD_TAG } \\ & \text { BSI_CARD_REMOVED } \\ & \text { BSI_NO_CARDSERVICE } \\ & \text { BSI_ACCESS_DENIED } \\ & \text { BSI_NO_MORE_SPACE } \\ & \text { BSI_IO_ERROR } \\ & \text { BSI_UNKNOWN_ERROR }\end{array}$




\section{F.4 Smart Card Cryptographic Provider Module Interface Definition}

\section{F.4.1 gscBsiGetChallenge()}

Purpose: $\quad$ Retrieves a randomly generated challenge from the card as the first step of a challenge-response authentication protocol between the client application and the card. The client subsequently encrypts the challenge using a symmetric key and returns the encrypted random challenge to the card through a call to gscBsiutilacquireContext () in the authValue instance field of the BSIAuthenticator object.

Prototype: $\quad$ public abstract byte[]
gscBsiGetChallenge (
hCard,
String AID
) throws gov.gsc.classes.BSIException;

Parameters: hCard: $\quad$ Card connection handle from gscBsiutilconnect () .

AID: $\quad$ Target container AID value. The AID shall be stored as an ASCII hexadecimal string.

Return Value: challenge: An array of bytes representing a random challenge returned from the card.

$\begin{array}{ll}\text { Return codes: } & \text { BSI_OK } \\ & \text { BSI_BAD_HANDLE } \\ & \text { BSI_BAD_AID } \\ & \text { BSI_SC_LOCKED } \\ & \text { BSI_CARD_REMOVED } \\ & \text { BSI_NO_CARDSERVICE } \\ & \text { BSI_UNKNOWN_ERROR }\end{array}$




\section{F.4.2 gscBsiSkilnternalAuthenticate()}

Purpose: $\quad$ Computes a symmetric key cryptogram in response to a challenge. In cases where the card reader authenticates the card, this function does not return a cryptogram. In these cases a BSI_TERMINAL_AUTH will be returned if the card reader successfully authenticates the card. BSI_ACCESS_DENIED is returned if the card reader fails to authenticate the card.

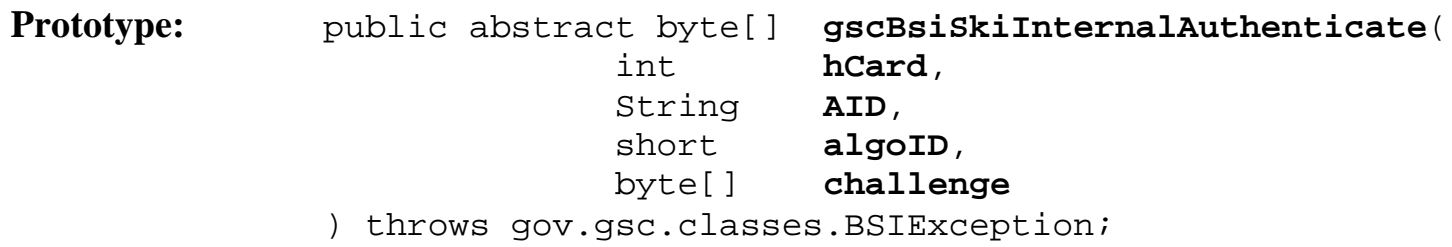

Parameters: hCard: $\quad$ Card connection handle from gscBsiutilConnect ().

AID: $\quad$ SKI provider module AID value. The AID shall be stored as an ASCII hexadecimal string.

algoID: Identifies the cryptographic algorithm that the card must use to encrypt the challenge. All conformant implementations shall, at a minimum, support the following algorithms: $D E S 3-E C B$

(Algorithm Identifier 0x81) and DES3-CBC (Algorithm Identifier 0x82). Implementations may optionally support other cryptographic algorithms.

challenge: Challenge generated by the client application and submitted to the card.

Return Value: cryptogram: The cryptogram computed by the card.

$\begin{array}{ll}\text { Return codes: } & \text { BSI_OK } \\ & \text { BSI_BAD_HANDLE } \\ & \text { BSI_BAD_AID } \\ & \text { BSI_SC_LOCKED } \\ & \text { BSI_BAD_PARAM } \\ & \text { BSI_BAD_ALGO_ID } \\ & \text { BSI_CARD_REMOVED } \\ & \text { BSI_NO_CARDSERVICE } \\ & \text { BSI_ACCESS_DENIED } \\ \text { BSI_UNKNOWN_ERROR }\end{array}$




\section{F.4.3 gscBsiPkiCompute()}

Purpose: $\quad$ Performs a private key computation on the message digest using the private key associated with the specified AID.

\begin{tabular}{|c|c|c|}
\hline Prototype: & $\begin{array}{r}\text { public abstract byte [] } \\
\text { int } \\
\text { String } \\
\text { short } \\
\text { byte [] }\end{array}$ & $\begin{array}{l}\text { gscBsipkiCompute } \\
\text { hCard, } \\
\text { AID, } \\
\text { algoID, } \\
\text { message }\end{array}$ \\
\hline
\end{tabular}

Parameters: hCard: $\quad$ Card connection handle from gscBsiutilconnect ().

AID: $\quad$ PKI provider module AID value. The AID shall be stored as an ASCII hexadecimal string.

algoID: Identifies the cryptographic algorithm that will be used to generate the signature. All conformant implementations shall, at a minimum, support RSA_NO_PAD (Algorithm Identifier 0xA3). Implementations may optionally support other algorithms.

message: $\quad$ The message digest to be signed.

Return Value: result: An array of bytes containing the signature.

Return codes: BSI_OK

BSI_BAD_HANDLE

BSI_BAD_AID

BSI_SC_LOCKED

BSI_BAD_PARAM

BSI_BAD_ALGO_ID

BSI_CARD_REMOVED

BSI_ACCESS_DENIED

BSI_NO_CARDSERVICE

BSI_UNKNOWN_ERROR 


\section{F.4.4 gscBsiPkiGetCertificate()}

Purpose: $\quad$ Reads the certificate from the card.
Prototype: public abstract byte[] gscBsipkiGetCertificate( int hCard,
Parameters: hCard: $\quad$ Card connection handle from gscBsiutilconnect () .
AID: $\quad$ PKI provider module AID value. The AID shall be stored as an ASCII hexadecimal string.

Return Value: certificate: An array of bytes containing the certificate.

$\begin{array}{ll}\text { Return codes: } & \text { BSI_OK } \\ & \text { BSI_BAD_HANDLE } \\ & \text { BSI_SC_LOCKED } \\ & \text { BSI_BAD_AID } \\ & \text { BSI_CARD_REMOVED } \\ & \text { BSI_NO_CARDSERVICE } \\ & \text { BSI_ACCESS_DENIED } \\ & \text { BSI_IO_ERROR } \\ \text { BSI_UNKNOWN_ERROR }\end{array}$




\section{F.4.5 gscBsiGetCryptoProperties()}

Purpose: $\quad$ Retrieves the Access Control Rules associated with the PKI provider module.

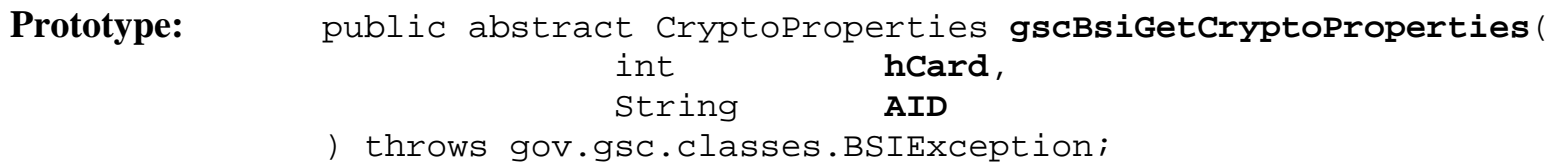

Parameters: hCard: $\quad$ Card connection handle from gscBsiutilconnect () .

AID :

AID of the PKI provider. The AID shall be stored as an ASCII hexadecimal string.

Return Value: cryptoprops : $\quad$ A CryptoProperties object defined as follows:

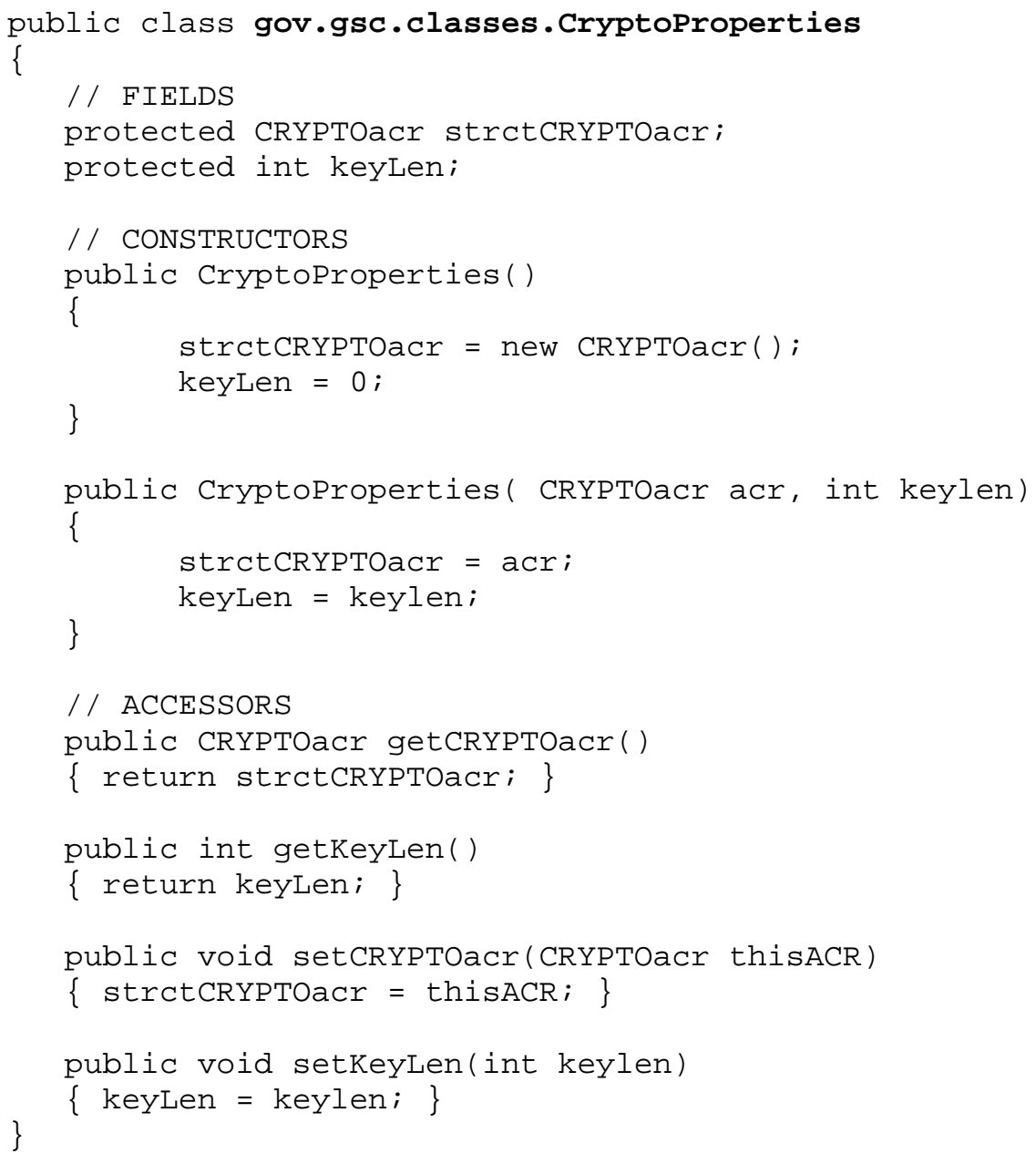

strctCRYPTOacr: Object indicating access control conditions for all operations. The BSIAcr structure is defined in Section F.3.3. The range of possible values for the instance fields of this object are defined in Table 3-2 (Section 3.1), and the allowable ACRs for each function in Table 3-4 (Section 3.2). 
keyIDOrReference contains the key identifier or reference for each access method contained in the ACR in order of appearance. authNb is the number of access methods logically combined in the ACR. ACRID is RFU and must be NULL (0x00) in this version. Note that the readValueACR member maps to the gscBsipkiGetCertificate () function.

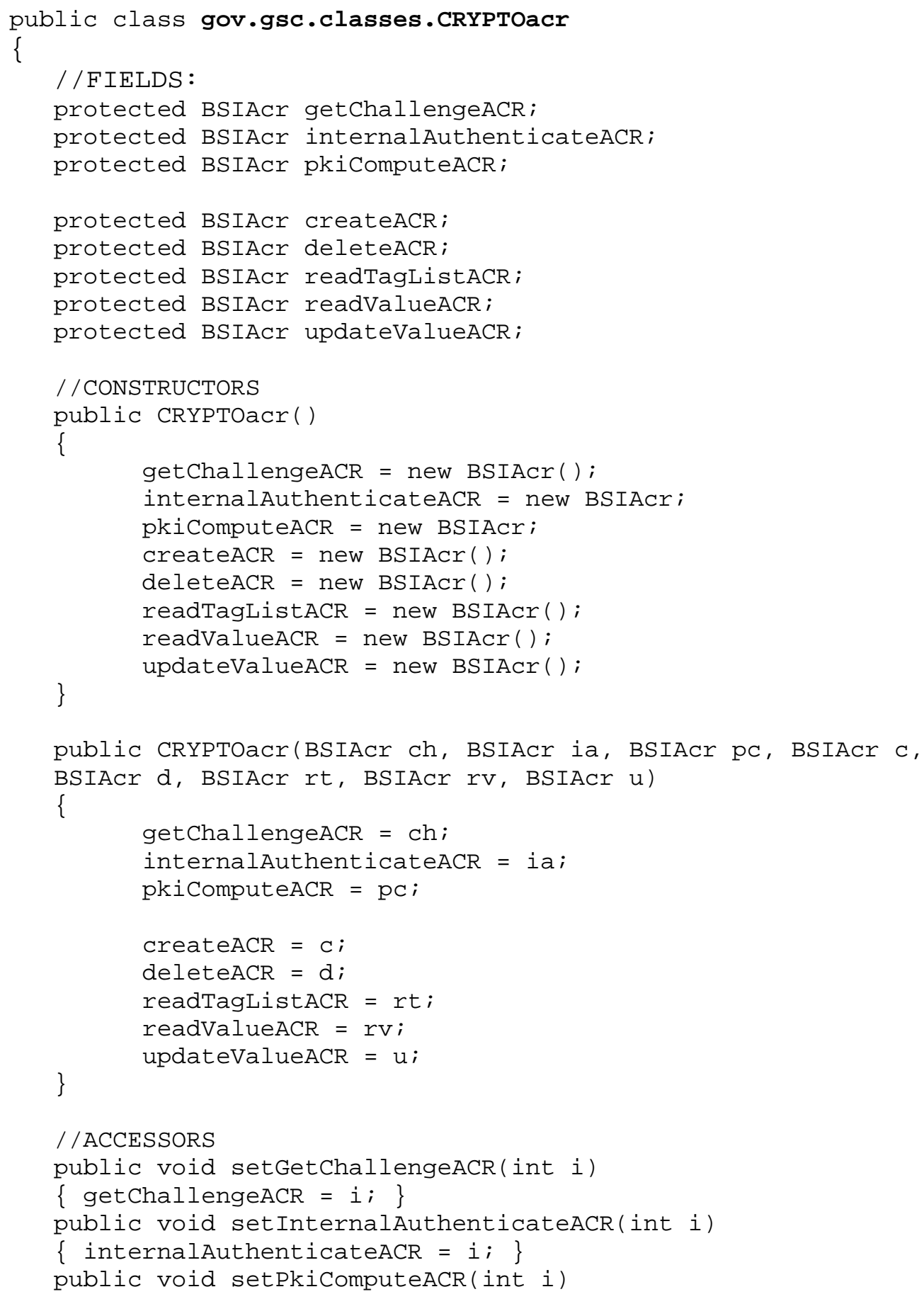




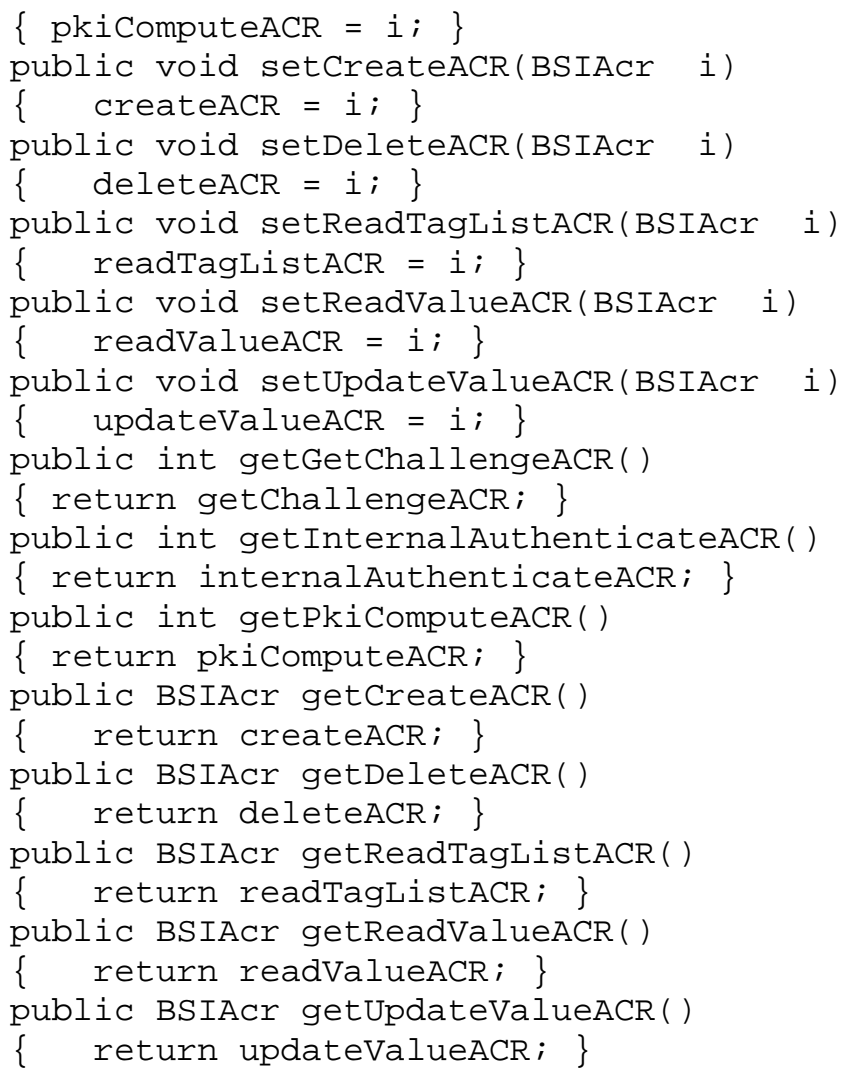

keyLen: $\quad$ Length of the private key managed by the PKI provider.

$\begin{array}{ll}\text { Return codes: } & \text { BSI_OK } \\ & \text { BSI_BAD_HANDLE } \\ & \text { BSI_BAD_AID } \\ & \text { BSI_SC_LOCKED } \\ & \text { BSI_CARD_REMOVED } \\ & \text { BSI_NO_CARDSERVICE } \\ & \text { BSI_UNKNOWN_ERROR }\end{array}$


NIST IR 6887 - 2003 EDITION, GSC-IS VERSION 2.1

\section{THIS PAGE INTENTIONALLY LEFT BLANK.}

F-38 


\section{Appendix G-Contactless Smart Card Requirements}

This appendix defines the requirements for GSC contactless smart cards, in accordance with the decisions of the Government Smart Card Interagency Advisory Board's Physical Access Interoperability Working Group (PAIWG). Contactless smart cards are often used in physical access control applications, but may also be used in the same environment as contact type cards. These requirements must therefore satisfy the following design goals:

GSC contactless cards should provide a minimum interoperability mechanism for cardholder identification in both physical access control and contact card type environments. This cardholder identification mechanism should use the same card edge functions (APDUs) and Data Models as those defined for GSC contact cards, to ensure interoperability with middleware designed for GSC contact cards.

The minimum interoperability mechanism for cardholder identification is to read a Security Equipment Integration Working Group (SEIWG)[SEIW] string from a fixed location using APDUs defined in the GSC virtual card edge interface.

\section{G.1 Card to Reader Interoperability}

GSC contactless cards and readers shall conform to ISO 14443 Parts 1 through 4[1444]. Cryptographic functionality is not required, but GSC contactless cards that implement cryptography shall use FIPS approved cryptographic algorithms in FIPS 140-2 [FIPS1] validated modules.

\section{G.2 Contactless Card Edge}

GSC contactless cards shall support two ISO 7816-4 [ISO4] APDUs required to select the SEIWG container/file and read the SEIWG string; READ BINARY and SELECT EF.

Note: The return code $0 \times 9000$ indicates command success, all other return codes indicate failure. Additional information on the READ BINARY APDU and SELECT EF APDUs can be found in Sections $\underline{5.1 .1 .2}$ and $\underline{5.1 .1 .4}$, respectively.

The Master File shall be automatically selected when a GSC contactless file system card is powered up. The applet that manages the SEIWG container shall be automatically selected on a GSC contactless Virtual Machine card at power up. SELECT MF and SELECT AID APDUs are therefore not required.

GSC contactless cards may optionally support other APDUs. These additional APDUs should be taken from the GSC file system card edge definitions in Chapter 5, to achieve maximum interoperability with middleware written for GSC contact cards.

\section{G.3 Data Model Requirements}

The SEIWG string is stored in a separate mandatory container/file in TLV format (EF 0007). This is necessary because host applications operating in a physical access control environment must be able to retrieve SEIWG strings quickly from a fixed location, and because no Access Control Rules are imposed on SEIWG container read operations. For file system cards, this file shall be a transparent file. 


\begin{tabular}{|l|l|l|l|}
\multicolumn{1}{|c|}{ Data Element (TLV) } & Tag & \multicolumn{1}{c|}{ Type } & \multicolumn{1}{c|}{ Max. Bytes } \\
\hline SEIWG Data & 30 & Fixed & $40^{*}$ \\
\hline Error Detection Code & FE & LRC & 1 \\
\hline
\end{tabular}

*The SEIWG data format is defined in [SEIW].

Only the FID component is mandated for the SEIWG File / Buffer. For container based implementations, the RID component of the AID is not defined by this specification.

All GSC contactless cards shall contain the SEIWG file as defined above as well as a valid Card Capability Container (see $\underline{\text { Chapter 6) }}$. Physical access control applications may elect not to read this container for reasons of efficiency. This is possible because GSC contactless cards directly implement a subset of the GSC virtual card edge interface and therefore require no APDU mapping. 


\section{Appendix H-Acronyms}

ACA

ACR

AID

ANSI

APDU

API

ASN.1

ATR

b

BSI

CAD

CCC

CEI

CHV

CLA

CT

DES

DES3

DES3-CBC

DES3-ECB

EDC

FID

FCI

GCA
Access Control Applet

Access Control Rule

Application Identifier

American National Standards Institute

Application protocol data unit

Applications Programming Interface

Abstract Syntax Notation One

Answer-to-Reset

Binary value

Basic Services Interface

Card Accepting Device

Card Capability Container

Card Edge Interface

Card Holder Verification

Class Byte of the Command Message

Capability Tuple

Data Encryption Standard

Triple Data Encryption Standard

Triple Data Encryption Standard in Cipher Block Chaining mode

Triple Data Encryption Standard in Electronic Codebook mode

Error Detection Code

File ID

File Control Information

Generic Container Applet 
NIST IR 6887 - 2003 EDITION, GSC-IS VERSION 2.1

GSC Government Smart Card, as defined in the Smart Access Common Identification Card Solicitation

GSC-IS Government Smart Card Interoperability Specification

h Hexadecimal value

IEC International Electrotechnical Commission

INS Instruction Byte of Command Message associated with the $T=0$ and $T=1$ protocol

ISO International Organization for Standardization

LEN or Len Length

LOUD Length of useful data

LRC Longitudinal Redundancy Check associated with the $T=1$ protocol

LSB Less Significant Byte

LSN Least significant nibbles

MAC Message Authentication Code

MSB Most Significant Byte

MSE Manage security environment command

OCF Open Card Framework

P1(2) Parameters used in the $T=0$ and $T=1$ protocol

PAIWG Physical Access Interoperability Working Group

PC/SC Personal Computer/Smart Card

PIN Personal Identification Number

PKI Public Key Infrastructure

PKCS Public Key Cryptography Standards

RFU Reserved for Future Use

RID Registerd Application Provider Identifier

SEIWG Security Enterprise Integration Working Group

SKI Symmetric Key Interface 


$\begin{array}{ll}\text { SPM } & \text { Service Provider Module } \\ \text { SPS } & \text { Service Provider Software } \\ \text { ST } & \text { Status Tuple } \\ \text { SW1(2) } & \text { Status Word1 (2) } \\ \text { T=0 } & \text { Character-oriented asynchronous half duplex transmission protocol } \\ \text { T=1 } & \text { Block-oriented asynchronous half duplex transmission protocol } \\ \text { TLV } & \text { Tag-Length-Value } \\ \text { USZ } & \text { Unsigned Zero-Terminated Character String } \\ \text { VCEI } & \text { Virtual Card Edge Interface } \\ \text { VM } & \text { Virtual Machine } \\ \text { VM CEI } & \text { Virtual Machine Card Edge Interface } \\ \text { XSI } & \text { Extended Service Interface(s) }\end{array}$

